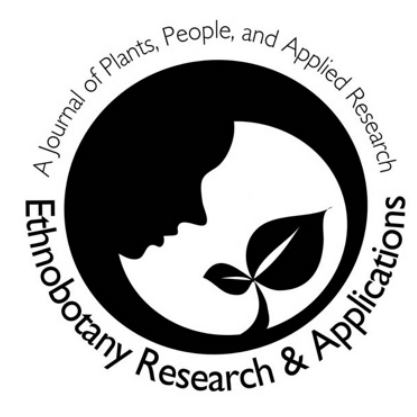

\title{
Indigenous knowledge of medicinal plants used by ethnic communities of South India
}

\author{
Santhosh Kumar JU, Krishna Chaitanya MJ, Andrew J \\ Semotiuk and Krishna V
}

\section{Databases and Inventories}

\begin{abstract}
Background: The information of therapeutic medicinal plants used by ethnic/indigenous communities in the south of India is constrained at best. These ethnomedicinal treatments constitute a critical local healthcare resource and potential for expanding research on phytomedicines of India. The present study aims to document and evaluate the traditional knowledge of medicinal plants as well as compare the distribution of knowledge and examine where research efforts are concentrated in order to gain a glimpse of current needs and future possibilities for research in the region.
\end{abstract}

Methods: This study conducted a literature review of multitude of ethnic/indigenous communities in regard to ethnobotany, medicinal plants, ethnic studies, and studies on traditional knowledge and included peerreviewed journal articles, and scientific reports published between 1980 and 2016 .

Results: Our study found a total of 2000 plant species belonging to 1033 genera and 215 families used in herbal preparations. Interestingly, only seven families contributed to the highest number of medicinal species and herbs used extensively for herbal preparations which include decoctions, infusions, tonics, and juice.

Conclusions: This multi-disciplinary, wide-scale study brought in data from a wide diversity of both plant species and ethnic communities. The study documents the rich wealth of indigenous knowledge and usage of medicinal plants for the treatment of various diseases and also these research highpoints the potential of continued research with these communities and highlights some areas where more research efforts could be directed. This also underlines the potential for future studies on drug screening and the need for conservation of biodiversity and traditional ecological knowledge practices.

Keywords: Ethnic group, indigenous communities, medicinal plants, traditional knowledge.

\begin{abstract}
Background
An ethnic/indigenous community or ethnicity is a category of people who identify with each other based on similarities, such as common ancestry, language, social, religion, traditions, and cultural or national experiences (Oxford Dictionaries 2013). There are about 370 million indigenous people spread across the globe (UNHR \& APF 2013, United Nations Report 2009) The Anthropological Survey of India under the 'People of India Project' reported 461 ethnic/indigenous communities (tribal communities) in India (Xaxa 1999). These communities, hereafter referred to as indigenous communities, encompass many varied lifestyles and traditions. They are unique because they use plants for medicinal purposes, based on ancestral traditions knowledge of medicinal treatments and therapies springs from a tradition that includes the use of herbs, shrubs, trees, or palms.

However, indigenous people with low socioeconomic status are believed to rely more on traditional medicine because of unaffordability and unavailability of healthcare services (Gaitonde et al. 2005). Erosion and deterioration of traditional medical knowledge can be observed in many cultures.
\end{abstract}

\section{Correspondence \\ Santhosh Kumar JU* \\ Krishna Chaitanya MJ \\ Andrew J Semotiuk \\ Krishna V}

Department of Post Graduate Studies and Research in Biotechnology, Jnanasahyadri, Kuvempu University, Shankaraghatta, Shimoga 577451, India

*Corresponding author: santhu.ju@gmail.com

Ethnobotany Research \& Applications 18:4 (2019) 
This leads to a loss in biocultural diversity as well as alternative primary healthcare options and leads for drug discovery (Farnsworth et al. 1985). Several publications have focused on the life of indigenous communities in India (Xaxa 1999). Recently, due to loss of biological resources, an increasingly globalized society, cultural homogenization, and the desire for modernization have resulted in a general decline of the cultural and hence medicinal properties of plants as well as the disappearance of traditions allowing the creation of medicines from plants (Balick 2007, Zent \& Zent 2007).

Plants have been used since ancient times to cure and heal throughout human history and now. In many parts of the world, people use plant substances to cure diseases. However, medicinal plant knowledge has been identified as particularly vulnerable to loss and degradation worldwide (Case et al. 2005, Phillips \& Gentry 1993). This stems from various possible causes such as increased reliance on biomedical healthcare, devaluation of the occupation of traditional herbal practitioner by younger generations, lack of cultural support and a push by some governmental programs to "modernize" medical practice. Community-based research in public health focuses on social, structural, and physical environmental inequities through active involvement of community members, organizational representatives, and researchers in all aspects of the research process (Israel et al. 1998).

This publication reports and analyzes results of an extensive survey with the goal of documenting information on medicinal plants used by South Indian indigenous communities. The data collected include the scientific names of plants used for medicinal therapies and their therapeutic applications. This examination is intended to fill a fast disappearing wellspring of data to disseminate and reinvigorate the tradition of ethnobotanical medicine, and to preserve and awareness of the rich biodiversity associated with ethnobotanical customs.

\section{Materials and methods}

\section{Ethnic communities present in India}

Using the Ministry of tribal affairs database (http://tribal.nic.in/) developed a list of indigenous communities and from this, generated the distribution map of all states (Figure 1). In this study, mainly focused on four south Indian states: Andhra Pradesh, Karnataka, Kerala and Tamil Nadu.

\begin{abstract}
Literature search on medicinal plants used in South Indian ethnic communities

Medicinal plants have been used from ancient times by various ethnic groups within their traditional knowledge systems. From the period of 1980 to 2016, peer-reviewed scientific studies used to document traditional medicinal plants used in south Indian ethnic groups. A literature search covered various electronic databases (ISI Web of Science, Science Direct (Scopus), and Google Scholar) using specific search terms such as "medicinal plants", "traditional", "ethnomedicine", "herbal medicine", and each indigenous group's name such as "Adiyan", "Barda", "Sholiga" etc.
\end{abstract}

\section{Types of knowledge documented}

The data collected included usage and knowledge of plants used including their family, species name, plant type (trees, herbs, shrubs, climbers and ferns). It also included methods of application, parts of the plant material (whole plants, leaves, bark, stem, roots) used in creating a medicinal product, and the use of the herbal medicines in men, women (including pregnant women), children, and infants (Table 1). The study also documented the disease or condition treated, such as malarial fevers, cough, cold, chest pain, intestinal parasitic infections, skinrelated diseases, respiratory diseases, typhoid fever, dyspepsia, dysentery, diabetes, itches, piles and jaundice, bites (insect, snake and scorpion), liver disease, asthma, cancer, cholera, other venereal diseases (Table 1).

Funding agency supported research In order to gain a glimpse at the trajectory of research and funding across India pertaining to indigenous communities and knowledge, gathered publication and funding information from articles of our literature search. For each publication, collected the year of publication, and funding agencies' support. These data provide the information of funding agency support related to ethnobotany. Based on this research, one could explore the remaining or understudied ethnic groups.

\section{Informant consensus factor ( $\left.F_{\mathrm{IC}}\right)$}

Informant consensus factor was calculated to find out the homogeneity in the information given in the reports. The $F_{I C}$ is calculated by the following formula (Bhat et al. 2013, Heinrich et al. 1998, Malla et al. 2015, Singh et al. 2012, Totter et al. 1986).

$$
F_{I C}=\frac{N_{u r}-N_{t}}{N_{u r}-1}
$$

Where $\mathrm{N}_{\mathrm{ur}}$ is the number of use report in a particular category of health by informants and $N_{t}$ is the number of taxa or species used to treat that category. 


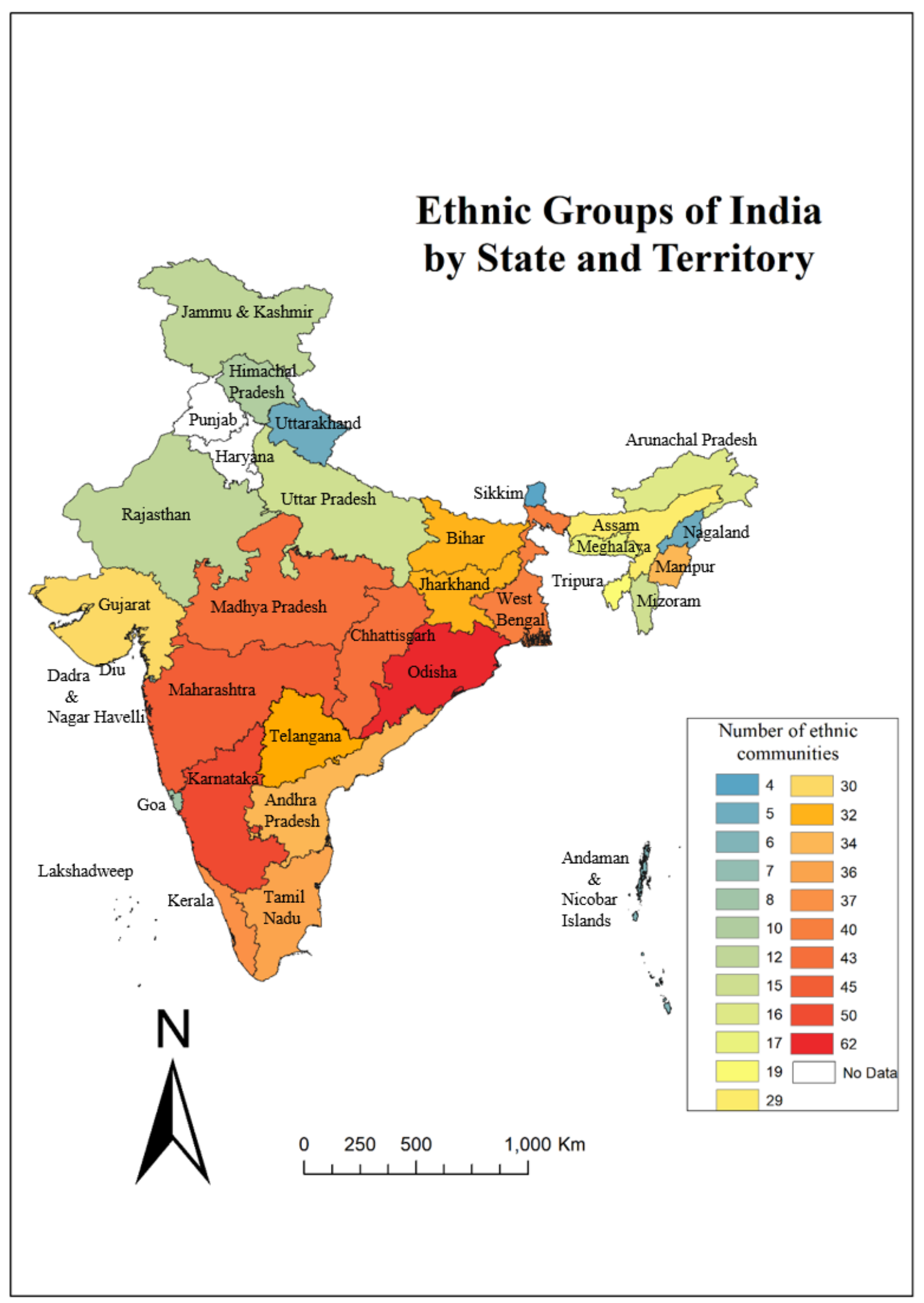

Figure 1. Distribution map of Ethnic communities in India. 
Table 1: Knowledge documented per species as revealed in the literature

\begin{tabular}{|c|c|c|c|c|c|}
\hline Scientific name & Habit & Parts used & Application & Treatment & References \\
\hline \multicolumn{6}{|l|}{ Acanthaceae } \\
\hline Acanthus ilicifolius $\mathrm{L}$. & Shrub & $\begin{array}{l}\text { Leaves, } \\
\text { root, fruit }\end{array}$ & $\begin{array}{l}\text { Malarial treatment; snake bite; rheumatism; anti } \\
\text { inflammatory }\end{array}$ & General & Dey and De 2012 \\
\hline Adhatoda beddomei C.B. Clarke & Herb & Leaves & Rheumatism; cough & General & Sudhadevi 1999 \\
\hline Andrographis affinis Nees & Herb & Leaves & Snake bite; scorpion bite and fever; skin problems & General & Alagesaboopathi 2012 \\
\hline Andrographis alata (Vahl) Nees & Herb & Whole plant & Snake bite; antipyretic and anti-inflammatory; wound; fever & General & Kshirsagar and Singh 2001 \\
\hline Andrographis echioides (L.) Nees & Herb & $\begin{array}{l}\text { Whole plant, } \\
\text { Leaves }\end{array}$ & $\begin{array}{l}\text { Fevers; cuts and wounds; liver disease; snake bite; } \\
\text { anthelmintic; itch; bronchitis; hair fall }\end{array}$ & General & $\begin{array}{l}\text { Jayanthi et al. 2012, Kumar et } \\
\text { al. 2007, Shanmugam et al. } \\
2012\end{array}$ \\
\hline Andrographis elongata (Vahl) T. Anderson & Herb & Whole plant & $\begin{array}{l}\text { Snake bite; diabetes and antidiabetic; antipyretic; jaundice; } \\
\text { fever; skin diseases; stomach ulcer; scorpion sting; } \\
\text { asthma; malarial fever; diuretic and constipation; ear and } \\
\text { muscular pains; anti-inflammatory; rabid dog bite; } \\
\text { dysentry; foot and mouth disease; constipation }\end{array}$ & $\begin{array}{l}\text { General; } \\
\text { animals }\end{array}$ & Alagesaboopathi 2012 \\
\hline Andrographis lineata Nees & $\begin{array}{l}\text { Stout erect } \\
\text { herb }\end{array}$ & $\begin{array}{l}\text { Leaves, } \\
\text { flower }\end{array}$ & Snakebite; diabetes; fever; cold & General & $\begin{array}{l}\text { Padma et al. 2016, Samy et al. } \\
2008\end{array}$ \\
\hline Andrographis macrobotrys Nees & Herb & Whole plant & Diarrhoea; snake bite; and antipyretic; skin problems & General & Alagesaboopathi 2012 \\
\hline Andrographis nallamalayana J.L. Ellis & $\begin{array}{l}\text { Low } \\
\text { decumbent } \\
\text { herb }\end{array}$ & Root & Mouth ulcers & General & Bhakshu et al. 2016 \\
\hline Andrographis neesiana Wight & Herb & Leaves & Aphrodisiac; jaundice; diuretic and fever. & General & Alagesaboopathi 2012 \\
\hline $\begin{array}{l}\text { Andrographis ovata (T. Anderson ex } \\
\text { Bedd.) Benth. and Hook. f. }\end{array}$ & Herb & Leaves & Diabetes and snake bite; skin diseases & General & Alagesaboopathi 2012 \\
\hline Andrographis paniculata (Burm. f.) Nees & Herb & Whole plant & $\begin{array}{l}\text { To treat malaria and malaria-like symptoms; dyspepsia; } \\
\text { dysentery; diabetes; itches; piles and jaundice; snake bite } \\
\text { and scorpion sting; wound; skin disease; fever; Liver } \\
\text { disease; hangover symptoms; warts; asthma; cancer; } \\
\text { cholera }\end{array}$ & $\begin{array}{l}\text { General; } \\
\text { children }\end{array}$ & $\begin{array}{l}\text { Padal et al. 2014, Raju 2008, } \\
\text { Ram et al. 2004, Rao et al. } \\
\text { 2006, Suneetha } 2011\end{array}$ \\
\hline Andrographis serpyllifolia (Vahl) Wight & Trailing herb & Whole plant & Cough; fever; wound; malaria & General & Rajendran et al. 2000 \\
\hline Asystasia gangetica (L.) T. Anderson & $\begin{array}{l}\text { Evergreen } \\
\text { herb }\end{array}$ & Whole plant & Rheumatism; fodder; leafy vegetable & General & Dansi et al. 2008 \\
\hline Avicennia marina (Forssk.) Vierh. & Tree & Leaves & Abortion; antimicrobial & $\begin{array}{l}\text { Pregnant } \\
\text { women }\end{array}$ & Murty and Venkaiah 2010 \\
\hline Barleria acuminata Wight ex Nees & Shrub & Whole plant & $\begin{array}{l}\text { Antiseptic; fever; respiratory diseases; tooth ache and joint } \\
\text { pain. }\end{array}$ & General & Kanthasamy Kalaichelvi 2016 \\
\hline Barleria buxifolia L. & Shrub & Leaves, root & $\begin{array}{l}\text { Cough and inflammations; Respiratory diseases; tooth } \\
\text { ache; joint pains; stomach pain }\end{array}$ & General & Pradheeps and Poyyamoli 2013 \\
\hline Barleria cristata L. & Under shrub & Whole plant & $\begin{array}{l}\text { Abortion; swellings; jaundice; wounds; sanke bite; tooth } \\
\text { ache }\end{array}$ & $\begin{array}{l}\text { General; } \\
\text { pregnant } \\
\text { women }\end{array}$ & Padma et al. 2016 \\
\hline Barleria gibsonii Dalzell & Under shrub & Root & Acidity & General & Kamble et al. 2008 \\
\hline Barleria mysorensis B. Heyne ex Roth & Shrub & Whole plant & Antiseptic; fever; cough & General & Dhivya and Kalaichelvi 2015 \\
\hline
\end{tabular}

Published 11 February 2019

http://dx.doi.org/10.32859/era.18.4.1-112 


\begin{tabular}{|c|c|c|c|c|c|}
\hline Barleria prattensis Santapau & Shrub & Whole plant & Paralysis & General & Jagtap et al. 2009 \\
\hline Barleria prionitis L. & Shrub & Whole plant & $\begin{array}{l}\text { To relieve toothache; asthma; urinary irritation; tooth } \\
\text { decay; gingival wounds; antiasthmatic; antiseptic; } \\
\text { rheumatism }\end{array}$ & General & $\begin{array}{l}\text { Bhat et al. 2012, Lingaraju et al. } \\
2013\end{array}$ \\
\hline $\begin{array}{l}\text { Blepharis integrifolia (L.f.) E. Mey. and } \\
\text { Drège ex Schinz }\end{array}$ & Herb & Whole plant & Bone fracture & General & Upadhya et al. 2012 \\
\hline $\begin{array}{l}\text { Blepharis maderaspatensis (L.) B. Heyne } \\
\text { ex Roth }\end{array}$ & Under shrub & Whole plant & $\begin{array}{l}\text { Wound healing; ulcers; nasal hemorhage;asthma; throat } \\
\text { inflammation; Ascitis; liver and spleen disorders; } \\
\text { Dysmenorrhoea; Urinary disorder; kidney stone; bone } \\
\text { fracture; scorpion bite; nervous disorder }\end{array}$ & General & $\begin{array}{l}\text { Kanthasamy Kalaichelvi 2016, } \\
\text { Ignacimuthu et al. 2008, } \\
\text { Subramanian } 2011\end{array}$ \\
\hline Blepharis repens (Vahl) Roth & Herb & $\begin{array}{l}\text { Root, young } \\
\text { parts }\end{array}$ & Heat and Summer stroke; oral health care & General & Hebbar et al. 2004 \\
\hline Clinacanthus nutans (Burm. f.) Lindau & Herb & Leaves & Snakebite & General & Samy et al. 2008 \\
\hline Crossandra infundibuliformis (L.) Nees & Shrub & Leaves & Eczema; scabies & General & Bhat et al. 2014 \\
\hline $\begin{array}{l}\text { Cynarospermum asperrimum (Nees) } \\
\text { Vollesen }\end{array}$ & Herb & Whole plant & Antibacterial activity; bone fracture & General & Ranjitha et al. 2016 \\
\hline Dicliptera paniculata (Forssk.) I. Darbysh. & Herb & Whole plant & Antidote; bone fractures & General & Ratnam and Raju 2008 \\
\hline Diotacanthus albiflorus (Bedd.) Benth. & Shrub & Leaves & Wound; heel cracks & General & Duraipandiyan et al. 2006 \\
\hline Dipteracanthus prostratus (Poir.) Nees & Herb & Whole plant & Hypoglycaemic; anticancer; ear-diseases & General & Jain et al. 2009 \\
\hline Dyschoriste nagchana (Nees) Bennet & Herb & Leaves & Vegetable & General & Dansi et al. 2008 \\
\hline Ecbolium viride (Forssk.) Alston & $\begin{array}{l}\text { Erect sub } \\
\text { shrub }\end{array}$ & Leaves & To reduce heat in the body and to cool the eye & General & Ayyanar and Ignacimuthu 2005 \\
\hline Elytraria acaulis (L.f.) Lindau & Herb & Root, leaves & Snakebite; tonic; insecticidal; wormicidal; cold; hip pain & General & $\begin{array}{l}\text { Dey and De 2012, Rao et al. } \\
2006\end{array}$ \\
\hline Eranthemum capense L. & Herb & Latex & Boils and blisters & General & Rao et al. 2006 \\
\hline Eranthemum roseum (Vahl) R. Br. & Herb & Root & Typhoid & General & Porte 2014 \\
\hline Graptophyllum pictum (L.) Griff. & Shrub & Root & Skin diseases & General & Harsha et al. 2003 \\
\hline Hemigraphis colorata W. Bull & Shrub & Leaves & For wound healing & General & Latheef et al. 2014 \\
\hline Hygrophila auriculata (Schumach.) Heine & Herb & Whole plant & $\begin{array}{l}\text { Migraine and headache; edema; anaemia; jaundice; } \\
\text { considered to be aphrodisiac; for impotency and seminal } \\
\text { weakness; vitality and good health; rheumatic arthritis; } \\
\text { male sexual debility; kidney stones }\end{array}$ & General & $\begin{array}{l}\text { Jain 2004, Pushpangadan and } \\
\text { Atal 1984, Pushpakarani and } \\
\text { Natarajan } 2014\end{array}$ \\
\hline Justicia adhatoda L. & Shrub & Whole plant & $\begin{array}{l}\text { Expectorant; insecticidal; wormicidal; tuberculosis; } \\
\text { bronchitis; veneral diseases; cough; asthma; snakebite; } \\
\text { asthma; fits; fever; antipyretic; antibacterial }\end{array}$ & General & $\begin{array}{l}\text { Chopda et al. 2009, Ramya et } \\
\text { al. 2009, Subramanian and } \\
\text { Ramakrishnan } 2011\end{array}$ \\
\hline Justicia betonica L. & $\begin{array}{l}\text { Gregarious } \\
\text { shrub }\end{array}$ & $\begin{array}{l}\text { Leaves, } \\
\text { flower }\end{array}$ & Vomiting; constipation; hair wash; finger joint abscess & General & Rao et al. 2006 \\
\hline Justicia diffusa Willd. & Herb & Leaves & Skin diseases; tooth ache & General & Ignacimuthu et al. 2008 \\
\hline Justicia gendarussa Burm. f. & Shrub & Root, leaves & $\begin{array}{l}\text { Inflammations; bronchitis; vaginal discharges; dyspepsia; } \\
\text { eye diseases; muscle pain; lumbago; headache; earache; } \\
\text { hemiplegia; hair growth; leucoderma; asthma; antiseptic; } \\
\text { haemo-static; nasal bleeding; bone fracture; injuries and } \\
\text { fever; jaundice; dysentery; rheumatism }\end{array}$ & $\begin{array}{l}\text { General; } \\
\text { women }\end{array}$ & $\begin{array}{l}\text { Chithra et al. 2016, Seraj et al. } \\
2013 \text {, Sugumaran et al. } 2013\end{array}$ \\
\hline Justicia glauca Rottler & Herb & Leaves & Snake bite & General & Savithramma et al. 2014 \\
\hline Justicia sumatrana Kurz & Herb & Leaves & Snake bite & General & Chander et al. 2015 \\
\hline
\end{tabular}

Published 11 February 2019

http://dx.doi.org/10.32859/era.18.4.1-112 
Ethnobotany Research and Applications

\begin{tabular}{|c|c|c|c|c|c|}
\hline Justicia tenella (Nees) T. Anderson & Herb & Leaves & Vegetable & General & Dansi et al. 2008 \\
\hline Justicia tranquebariensis L.f. & Under shrub & Leaves & Small pox & General & Jayanthi et al. 2012 \\
\hline Justicia wynaadensis B. Heyne & Herb & $\begin{array}{l}\text { Leaves, } \\
\text { stem }\end{array}$ & Asthma; imunity & General & Lingaraju et al. 2013 \\
\hline Lepidagathis cristata Willd. & Under shrub & Whole plant & Skin diseases; snake bite & General & Dey and De 2012 \\
\hline Nelsonia canescens (Lam.) Spreng. & Herb & Root & - & - & Chopda and Mahajan 2009 \\
\hline Peristrophe bicalyculata (Retz.) Nees & Herb & $\begin{array}{l}\text { Leaves, } \\
\text { stem }\end{array}$ & Anti-inflammatory; anti-microbial; asthma & General & Kanthasamy Kalaichelvi 2016 \\
\hline Phaulopsis imbricata (Forssk.) Sweet & Herb & Leaves & Urinary problems; skin infection & General & Kanthasamy Kalaichelvi 2016 \\
\hline $\begin{array}{l}\text { Phlogacanthus thyrsiformis (Roxb. ex } \\
\text { Hardw.) Mabb. }\end{array}$ & Herb & Leaves & Contraceptive & General & Das et al. 2014 \\
\hline Rhinacanthus nasutus (L.) Kurz & Herb & $\begin{array}{l}\text { Leaves, } \\
\text { root, flower }\end{array}$ & $\begin{array}{l}\text { Leucoderma; hepatoprotective; antipyretic; cough; } \\
\text { wounds(live stock); snakebite; eye disease }\end{array}$ & $\begin{array}{l}\text { General; } \\
\text { veterinary }\end{array}$ & Bhat et al. 2014 \\
\hline Ruellia patula Jacq. & Herb & - & Remedy against deadly poison of tiger spider & General & Prakash et al. 2008 \\
\hline Ruellia prostrata Poir. & Herb & Leaves & Fever; asthma; cold; hair fall & General & Ayyanar and Ignacimuthu 2005 \\
\hline Ruellia tuberosa L. & Herb & Leaves & Severe body pain & General & Seraj et al. 2013 \\
\hline Rungia linifolia Nees & Herb & Whole plant & Ulcer & General & Ignacimuthu et al. 2008 \\
\hline Strobilanthes ciliata T. Anderson & Herb & Leaves & Toothache; swellings; fever & General & Chithra et al. 2016 \\
\hline Thunbergia alata Bojer ex Sims & Herb & Leaves, root & Menorrhagia; irregular menstruation; treats rotten nail & $\begin{array}{l}\text { General; } \\
\text { women }\end{array}$ & Rao et al. 2011 \\
\hline Thunbergia erecta (Benth.) T. Anderson & Climber & Leaves & Whooping cough; hydrophobia & General & Rao et al. 2006 \\
\hline Thunbergia fragrans Roxb. & Climber & Root, leaves & Snakebite; rheumatism; cough & General & $\begin{array}{l}\text { Rajan et al. 1997, Sarvalingam } \\
\text { et al. } 2011\end{array}$ \\
\hline $\begin{array}{l}\text { Thunbergia grandiflora (Roxb. ex Rottl.) } \\
\text { Roxb. }\end{array}$ & Climber & Whole plant & Snakebite & General & Dey and De 2012 \\
\hline Thunbergia laurifolia Lindl. & Climber & Leaves & Cuts; wounds & General & Prasad et al. 2008 \\
\hline Thunbergia tomentosa Wall. ex Nees & Climber & Whole plant & Eye diseases & General & Puravankara and Gopal 2012 \\
\hline \multicolumn{6}{|l|}{ Achariaceae } \\
\hline Gynocardia odorata R. Br. & Tree & Leaves & Tooth decay & General & Kumar 2014 \\
\hline $\begin{array}{l}\text { Hydnocarpus pentandrus (Buch. -Ham.) } \\
\text { Oken }\end{array}$ & $\begin{array}{l}\text { Medium } \\
\text { sized tree }\end{array}$ & Seed & Skin diseases; wound; scabies & General & Chithra et al. 2016 \\
\hline Hydnocarpus wightianus Blume & Shrub & Fruit, seed & $\begin{array}{l}\text { Boils; ulcers; sores; leprosy; rheumatic arthritis; diabetes; } \\
\text { swelling and skin diseases }\end{array}$ & General & Priya and Gopalan 2014 \\
\hline \multicolumn{6}{|c|}{ 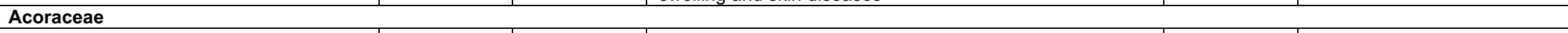 } \\
\hline Acorus calamus $\mathrm{L}$. & Herb & Whole plant & $\begin{array}{l}\text { Chronic urticaria; snakebite; stomach ache; poisonous } \\
\text { bites; epilepsy; cough; wound; asthma; as a substitute for } \\
\text { ginger; malaria; obesity diseases; body pain; oral health } \\
\text { care }\end{array}$ & General & $\begin{array}{l}\text { Chopda and Mahajan 2009, } \\
\text { Sharma et al. 2013, Valsaraj et } \\
\text { al. } 1997\end{array}$ \\
\hline \multicolumn{6}{|l|}{ Actinidiaceae } \\
\hline $\begin{array}{l}\text { Actinidia arguta (Siebold and Zucc.) } \\
\text { Planch. ex Miq. }\end{array}$ & Tree & - & Anti-lipase & General & Zhang et al. 2013 \\
\hline Actinidia chinensis Planch. & Tree & Fruit & Laxative (laxative) & General & Parada et al. 2009 \\
\hline
\end{tabular}




\begin{tabular}{|c|c|c|c|c|c|}
\hline Trianthema portulacastrum $\mathrm{L}$. & Herb & Whole plant & $\begin{array}{l}\text { Cough; asthma; scorpion sting; rheumatism; liver } \\
\text { diseases; vegetable }\end{array}$ & General & $\begin{array}{l}\text { Dansi et al. 2008, Rao et al. } \\
2006\end{array}$ \\
\hline Zaleya decandra (L.) Burm. f. & Herb & Whole plant & $\begin{array}{l}\text { Asthma; snake bite; muscle strengthening; gastrointestinal } \\
\text { disorders; toothache; heel and palm cracks; diabetes; liver } \\
\text { damage }\end{array}$ & General & $\begin{array}{l}\text { Kumar et al. 2007, Padma et al. } \\
2016\end{array}$ \\
\hline \multicolumn{6}{|l|}{ Alismataceae } \\
\hline Sagittaria sagittifolia L. & Herb & Whole plant & Amenorrhoea; abnormal menstruation & Women & Patel 2010 \\
\hline \multicolumn{6}{|l|}{ Amaranthaceae } \\
\hline Achyranthes aspera L. & $\begin{array}{l}\text { Erect or } \\
\text { straggling } \\
\text { herb }\end{array}$ & Whole plant & $\begin{array}{l}\text { Diuretic; ease for child birth; epilepsy; antidote to } \\
\text { poisonous bites; piles; cough; mentally retarded; } \\
\text { Diverticulosis and diverticulitis; tumor; digestion; } \\
\text { leucoderma; asthma; antifertility; anaemia; gastrointestinal } \\
\text { disorder; eczema; jaundice; menstural disorder; abortion }\end{array}$ & $\begin{array}{l}\text { Women; } \\
\text { pregnant } \\
\text { women; } \\
\text { General }\end{array}$ & Rajan et al. 1997 \\
\hline Achyranthes bidentata Blume & Herb & Whole plant & Boils; snakebite; General & General & Dey et al. 2012, Raju 2008 \\
\hline Achyranthes porphyristachya Wall. & Herb & Root & Wounds & General & Dey and De 2012 \\
\hline Aerva javanica (Burm. f.) Juss. & Herb & $\begin{array}{l}\text { Flowers, } \\
\text { seed }\end{array}$ & Rheumatism; head ache; swellings & General & $\begin{array}{l}\text { Dhivya and Kalaichelvi 2015, } \\
\text { Ram et al. } 2016\end{array}$ \\
\hline Aerva lanata (L.) Juss. & Herb & Whole plant & $\begin{array}{l}\text { Snake- bite; cough; sore throat; diuretic and diabetes; } \\
\text { typhoid; renal calculi; earache; anthelmintic; drowsiness; } \\
\text { asthma; headache; herpes; fever; demulcent; kidney } \\
\text { stones }\end{array}$ & General & $\begin{array}{l}\text { Krishna et al. 2011, Padal et al. } \\
\text { 2014, Reddy et al. 2008, Samy } \\
\text { et al. } 2008\end{array}$ \\
\hline Aerva sanguinolenta (L.) Blume & Herb & Whole plant & Intestinal worms; fever & General & Kosalge and Fursule 2009 \\
\hline Allmania nodiflora (L.) R. Br. ex Wight & $\begin{array}{l}\text { Straggling } \\
\text { herb }\end{array}$ & Leaves & Digestive problems & General & Prabakaran et al. 2013 \\
\hline Alternanthera brasiliana (L.) Kuntze & Herb & Leaves & Vegetable & General & Dansi et al. 2008 \\
\hline Alternanthera philoxeroides (Mart.) Griseb. & Herb & Leaves & Hair tonic; fever & General & Prasad et al. 2008 \\
\hline Alternanthera pungens Kunth & Herb & Whole plant & Anti-oxidant; night blindness & General & Jain et al. 2009 \\
\hline Alternanthera sessilis (L.) R. Br. ex DC. & Herb & Whole plant & $\begin{array}{l}\text { Lactagogue; stomach ache; snake bite; aphthae; } \\
\text { diarrhoea; edible; increase haemoglobin; swelling; night } \\
\text { blindness; hair tonic; Anti ulceratives; excessive acidity in } \\
\text { the body }\end{array}$ & General & $\begin{array}{l}\text { Bhat et al. 2012, Puravankara } \\
\text { and Gopal } 2012\end{array}$ \\
\hline Amaranthus blitum L. & Herb & Root, leaves & Snakebite; headache & General & Dey and De 2012 \\
\hline Amaranthus caudatus $\mathrm{L}$. & Herb & Whole plant & $\begin{array}{l}\text { Pulmonary congestion; Astringent; anthelmentic and } \\
\text { diuretic. }\end{array}$ & General & Kayani et al. 2014 \\
\hline Amaranthus polygamus L. & Herb & $\begin{array}{l}\text { Leaves, } \\
\text { root, seed }\end{array}$ & Good detoxin; Aphrodisiac & General & Dhivya and Kalaichelvi 2015 \\
\hline Amaranthus spinosus $\mathrm{L}$. & Herb & Whole plant & $\begin{array}{l}\text { For improving digestion; bruises; abscesses; burns; } \\
\text { wounds and inflammation; snake bite; stomach ulcer; } \\
\text { indigestion; stomach complaints and kidney stone; urinary } \\
\text { tract infection; high blood pressure; constipation; piles; hair } \\
\text { fall; and increasing lactation; diuretic; fever }\end{array}$ & $\begin{array}{l}\text { Women; } \\
\text { General }\end{array}$ & $\begin{array}{l}\text { Dansi et al. 2008, Ignacimuthu } \\
\text { et al. 2008, Shanmugam et al. } \\
2012\end{array}$ \\
\hline Amaranthus tricolor $\mathrm{L}$. & Herb & Whole plant & $\begin{array}{l}\text { Blood purifier; anemia; dandruff; ringworm; mensturation } \\
\text { pain; leucorrhea; increases breast milk; kidney stone; } \\
\text { constipation; ear problem; ulcers; indigestion }\end{array}$ & General & $\begin{array}{l}\text { Bhat et al. 2012, Horo and } \\
\text { Topno 2015, Shanmugam et al. } \\
2012\end{array}$ \\
\hline
\end{tabular}

Published 11 February 2019

http://dx.doi.org/10.32859/era.18.4.1-112 


\begin{tabular}{|c|c|c|c|c|c|}
\hline Amaranthus viridis $\mathrm{L}$. & Herb & Whole plant & $\begin{array}{l}\text { Diuretic; purgative enema during stomach troubles; snake } \\
\text { bite; stomach ache; flu; cold; Erysipelas; scorpion sting }\end{array}$ & General & $\begin{array}{l}\text { Ignacimuthu et al. 2008, Kayani } \\
\text { et al. } 2014\end{array}$ \\
\hline Celosia argentea L. & Herb & Whole plant & $\begin{array}{l}\text { Mouth sores; eye diseases; diarrhoea; orally for ovarian } \\
\text { and uterus diseases; dysentery; sores; tonic; tuberculosis; } \\
\text { stomach ache; skin disease; scorpion bite }\end{array}$ & $\begin{array}{l}\text { General; } \\
\text { women }\end{array}$ & $\begin{array}{l}\text { Madhu and Swamy 2010, } \\
\text { Gairola et al. } 2013\end{array}$ \\
\hline Celosia polygonoides Retz. & Herb & Whole plant & $\begin{array}{l}\text { Wound healing; cold; heaviness of head; diarrhea; snake- } \\
\text { bite;jaundice and reduce body heat }\end{array}$ & General & Dhivya and Kalaichelvi 2015 \\
\hline Chenopodium album $\mathrm{L}$. & Herb & Whole plant & $\begin{array}{l}\text { As purgative; antidote; tuberculosis; edible; insecticidal; } \\
\text { wormicidal; aphrodisiac }\end{array}$ & General & Chopda and Mahajan 2009 \\
\hline Chenopodium murale L. & Herb & Leaves & Fever and body pain due to fever & General & Puravankara and Gopal 2012 \\
\hline Cyathula prostrata (L.) Blume & Herb & Whole plant & Diarrhoea; scabies; gynaecological disorders & $\begin{array}{l}\text { General; } \\
\text { women }\end{array}$ & Lingaraju et al. 2013 \\
\hline Digera muricata (L.) Mart. & $\begin{array}{l}\text { Profusely } \\
\text { branched } \\
\text { herb }\end{array}$ & $\begin{array}{l}\text { Flower, } \\
\text { seed, leaves }\end{array}$ & $\begin{array}{l}\text { Laxative; urinary troubles; boils; vegetable; fever; gas } \\
\text { trouble; body swelling }\end{array}$ & $\begin{array}{l}\text { General; } \\
\text { women }\end{array}$ & Jain et al. 2009 \\
\hline $\begin{array}{l}\text { Dysphania ambrosioides (L.) Mosyakin } \\
\text { and Clemants }\end{array}$ & Herb & Whole plant & $\begin{array}{l}\text { To remove the body odour; insecticidal; wormicidal; } \\
\text { vegetable; skin swellings; dysmenorrhea; Anthelmintic; } \\
\text { skin allergy; }\end{array}$ & $\begin{array}{l}\text { General; } 1 \\
\text { month baby }\end{array}$ & $\begin{array}{l}\text { Bhardwaj et al. 2011, Dansi et } \\
\text { al. 2008, Kshirsagar and Singh } \\
2001\end{array}$ \\
\hline Gomphrena celosioides Mart. & Herb & Leaves & Vegetable & General & Sharma et al. 2013 \\
\hline Gomphrena serrata L. & Herb & Root & Stomach ulcers & General & Rao et al. 2006 \\
\hline Halosarcia indica (Willd.) Paul G. Wilson & Shrub & Leaves & Edible & General & Gavali and Sharma 2004 \\
\hline Iresine herbtii Hook. & Herb & Leaves & Tonic; post-labor tonic & Women & Srithi et al. 2009 \\
\hline Pandiaka involucrata (Moq.) B.D. Jacks. & Herb & Leaves & Vegetable & General & Dansi et al. 2008 \\
\hline Psilotrichum nudum Wight & Herb & Leaves & Fever; Cold & General & Dhivya and Kalaichelvi 2015 \\
\hline Pupalia lappacea (L.) Juss. & Herb & Leaves & Vegetable; wound & General & Shanmugam et al. 2012 \\
\hline Trichuriella monsoniae (L.f.) Bennet & Herb & Leaves & Abdominal pains & General & Rao et al. 2006 \\
\hline \multicolumn{6}{|l|}{ Amaryllidaceae } \\
\hline Allium carolinianum DC. & Herb & Whole plant & Flu; cough & General & Kayani et al. 2014 \\
\hline Allium cepa L. & Herb & Bulbs & $\begin{array}{l}\text { Chicken pox fever; diabetes; to expel poison by vomiting; } \\
\text { boils; blisters; cuts; stomachache; bronchitis; } \\
\text { Amenorrhoea and Abnormal Menstruation; impotency; } \\
\text { contraceptive; snake bite; aphrodisiac; rheumatism }\end{array}$ & $\begin{array}{l}\text { General, } \\
\text { Women }\end{array}$ & $\begin{array}{l}\text { Bhat et al. 2012, Ignacimuthu et } \\
\text { al. 2008, Kadhirvel et al. 2010, } \\
\text { Patel } 2010\end{array}$ \\
\hline Allium hookeri Thwaites & Herb & $\begin{array}{l}\text { Leaves, } \\
\text { rhizome }\end{array}$ & Skin diseases; Veterinary; bone fracture; & General & Ruba and Mohan 2016 \\
\hline Allium sativum $\mathrm{L}$. & Herb & $\begin{array}{l}\text { Bulb, } \\
\text { leaves, } \\
\text { rhizome, } \\
\text { pod }\end{array}$ & $\begin{array}{l}\text { Enhance conception; weight reduction; dysentery; heat } \\
\text { diseases and earache; diabetes; epilepsy; tooth deacy; } \\
\text { bronchitis; earache; warts; whooping cough; oral health } \\
\text { care; Hyperacidity; respiratorty disorders; increase } \\
\text { lactation; bone fracture }\end{array}$ & General & $\begin{array}{l}\text { Murty and Venkaiah 2010, } \\
\text { Parada et al. 2009, Sharma et } \\
\text { al. } 2013\end{array}$ \\
\hline Allium victorialis $\mathrm{L}$. & Herb & Whole plant & Asthma; respiratory problems & General & Kayani et al. 2014 \\
\hline Crinum asiaticum $\mathrm{L}$. & Herb & $\begin{array}{l}\text { Bark, tuber, } \\
\text { leaves }\end{array}$ & $\begin{array}{l}\text { Wounds; snake bite; skin diseases; arthritis; rheumatic } \\
\text { pain; eczema }\end{array}$ & General & Bhuvaneswari et al. 2015 \\
\hline Crinum latifolium $\mathrm{L}$. & Herb & Tubers, bulb & $\begin{array}{l}\text { Asthma; cough; bronchial congestion; chest complaints } \\
\text { and mucous discharge; against dysentery and black } \\
\text { motion; ring worm; cures cramps and dryness; swellings }\end{array}$ & General & Priya and Gopalan 2014 \\
\hline
\end{tabular}

Published 11 February 2019

http://dx.doi.org/10.32859/era.18.4.1-112 


\begin{tabular}{|c|c|c|c|c|c|}
\hline Hippeastrum puniceum (Lam.) Voss & Herb & - & - & General & Chakraborty et al. 2012 \\
\hline \multicolumn{6}{|l|}{ Amblystegiaceae } \\
\hline Cratoneuron filicinum (Hedw.) Spruce & Hypnales & - & Heart disease & General & Chandra et al. 2016 \\
\hline \multicolumn{6}{|l|}{ Anacardiaceae } \\
\hline Anacardium occidentale L. & Tree & $\begin{array}{l}\text { Endosperm, } \\
\text { seed, fruit } \\
\text { pulp, } \\
\text { cashew } \\
\text { shell, shell } \\
\text { oil, nut, fruit, } \\
\text { bark, seed } \\
\text { coat oil, } \\
\text { root, stem }\end{array}$ & $\begin{array}{l}\text { Snake-bite; dental cavities; tooth problems; edible; corns; } \\
\text { warts; impotency; jaundice; fever; heel cracks; skin } \\
\text { disorder; antidiarrhoeal; Anthelmintic; astringent; irritant; } \\
\text { purgative; rubefacient and vesicant }\end{array}$ & $\begin{array}{l}\text { General; } \\
\text { women; } \\
\text { men }\end{array}$ & $\begin{array}{l}\text { Bhat et al. 2012, Bosco and } \\
\text { Arumugam 2012, Ignacimuthu et } \\
\text { al. } 2008 \text {, Suganthi and Libina } \\
2015\end{array}$ \\
\hline Anaxagorea luzonensis A. Gray & Shrub & Leaves & Cracks of foot & General & Prasad et al. 2008 \\
\hline Ancistrocladus attenuatus Dyer & $\begin{array}{l}\text { Scandent } \\
\text { shrub }\end{array}$ & Whole plant & Jaundice & General & Prasad et al. 2008 \\
\hline Annona muricata L. & Tree & $\begin{array}{l}\text { Stem, bark, } \\
\text { leaves }\end{array}$ & Abortifacient & $\begin{array}{l}\text { Pregnant } \\
\text { women }\end{array}$ & Awate and Gaikwad 2014 \\
\hline Annona reticulata $\mathrm{L}$. & Tree & $\begin{array}{l}\text { Seed, } \\
\text { leaves, fruit }\end{array}$ & Abortion; edible; diabetes; lice eradication & $\begin{array}{l}\text { Pregnant } \\
\text { women; } \\
\text { General }\end{array}$ & $\begin{array}{l}\text { Rao et al. 2006, Murty and } \\
\text { Venkaiah } 2010\end{array}$ \\
\hline Annona senegalensis Pers. & Tree & - & Dysentry; malignant tumor & General & Dansi et al. 2008 \\
\hline Annona squamosa L. & Tree & Whole plant & $\begin{array}{l}\text { To remove lice; for external healing; bronchitis; snake bite; } \\
\text { fever; worm infection; cuts; painful joints; antidiabetic; } \\
\text { rheumatism; animal wounds; abortion; hepatoprotective; } \\
\text { vermifuge; bone fracture; tooth worms; tumor; cough }\end{array}$ & $\begin{array}{l}\text { General; } \\
\text { cattle; } \\
\text { pregnant } \\
\text { women; } \\
\text { children }\end{array}$ & Kumar and Pullaiah 1999 \\
\hline Buchanania axillaris (Desr.) Ramamoorthy & Tree & $\begin{array}{l}\text { Flower, } \\
\text { gum, leaves }\end{array}$ & $\begin{array}{l}\text { Wounds treatment; chest and body pains; edible; stomach } \\
\text { ache }\end{array}$ & General & Murthy et al. 2008 \\
\hline $\begin{array}{l}\text { Buchanania cochinchinensis (Lour.) M.R. } \\
\text { Almeida }\end{array}$ & Tree & $\begin{array}{l}\text { Oil, flower, } \\
\text { fruit, seed, } \\
\text { bark }\end{array}$ & $\begin{array}{l}\text { For baldness; typhoid; rheumatic pain; joint disease; chest } \\
\text { pain; diarrhoea; skin diseases }\end{array}$ & General & $\begin{array}{l}\text { Dey and De 2012, Pushpakarani } \\
\text { and Natarajan } 2014\end{array}$ \\
\hline Holigarna grahamii (Wight) Kurz & Tree & Bark & Bone fracture & General & Upadhya et al. 2012 \\
\hline Lannea coromandelica (Houtt.) Merr. & Tree & Whole plant & $\begin{array}{l}\text { Fever; asthma; body pain; bone fracture; cuts; wound; } \\
\text { snake bite; centipede bite; dental caries; rheumatism }\end{array}$ & $\begin{array}{l}\text { General; } \\
\text { veterinary }\end{array}$ & $\begin{array}{l}\text { Manikandan and Lakshmanan } \\
2014\end{array}$ \\
\hline Mangifera andamanica King & Tree & Bark & Wound swellings & General & Prasad et al. 2008 \\
\hline Mangifera indica L. & Tree & Whole plant & $\begin{array}{l}\text { Malaria; toothache; asthma; bleeding; ulcer; frequent } \\
\text { urination; cholera; delivery pain; boils; blisters; wound; anti } \\
\text { diabetic; snakebite; chronic dysentery; liver diseases; } \\
\text { contraceptive; anifertility; mouth sores; rheumatism; } \\
\text { urethritis; diptheria; diarrhoea; pruritis; scabies; skin } \\
\text { diseases; antibacterial }\end{array}$ & $\begin{array}{l}\text { General; } \\
\text { pregnant } \\
\text { women }\end{array}$ & $\begin{array}{l}\text { Arjunan et al. 2016, Dey and De } \\
\text { 2012, Jain 2004, Rao et al. } 2006\end{array}$ \\
\hline Parishia insignis Hook. f. & Tree & Fruit & Cough & General & Prasad et al. 2008 \\
\hline Pistacia atlantica Desf. & Tree & $\begin{array}{l}\text { Leaves, } \\
\text { gum }\end{array}$ & Cough & General & Kayani et al. 2014 \\
\hline
\end{tabular}

Published 11 February 2019

http://dx.doi.org/10.32859/era.18.4.1-112 


\begin{tabular}{|c|c|c|c|c|c|}
\hline Pistacia chinensis Bunge & Tree & Whole plant & Asthma; cough; phlegm & General & Kayani et al. 2014 \\
\hline Rhus mysorensis G. Don & Shrub & Fruit & Edible & General & Bhagat et al. 2016 \\
\hline Rhus parviflora Roxb. & Shrub & Fruit, leaves & Laxative; cholera & General & Wagh and Jain 2015 \\
\hline Semecarpus anacardium L.f. & Herb & $\begin{array}{l}\text { Seed, fruit, } \\
\text { latex, bark, } \\
\text { oil }\end{array}$ & $\begin{array}{l}\text { Skin diseases; abortion; remedy for phlegm; ulcers; } \\
\text { rheumatism; leprosy; piles; expells worms; health tonic; } \\
\text { hair growth; brain development; vitality; antidiarrhoeal; } \\
\text { eczema; contraceptive; arthritis; cough; antibacterial; } \\
\text { infertility; muscle pain; sores; sprains; tooth infection }\end{array}$ & $\begin{array}{l}\text { Pregnant } \\
\text { women; } \\
\text { general }\end{array}$ & $\begin{array}{l}\text { Bhandary et al. 1995, Dey et al. } \\
\text { 2012, Jain 2004, Murty and } \\
\text { Venkaiah 2010, Ram et al. } \\
\text { 2004, Reddy et al. } 2008\end{array}$ \\
\hline Semecarpus kurzii Engl. & Herb & Bark & Leprosy & General & Prasad et al. 2008 \\
\hline Spondias pinnata (L.f.) Kurz & Tree & $\begin{array}{l}\text { Fruit, bark, } \\
\text { root }\end{array}$ & Dysentry; diaherroea; antibacterial; fever; boils; vegetable & General & Ajesh et al. 2012 \\
\hline \multicolumn{6}{|l|}{ Annonaceae } \\
\hline $\begin{array}{l}\text { Artabotrys zeylanicus Hook. f. and } \\
\text { Thomson }\end{array}$ & $\begin{array}{l}\text { Climbing } \\
\text { shrub }\end{array}$ & Flower & Vomiting & General & Yesodharan and Sujana 2007 \\
\hline Friesodielsia fornicata (Roxb.) D. Das & $\begin{array}{l}\text { Climbing } \\
\text { shrub }\end{array}$ & Root & Excess of heat & General & Prasad et al. 2008 \\
\hline Goniothalamus macranthus (Kurz) Boerl. & $\begin{array}{l}\text { Shrub or } \\
\text { small tree }\end{array}$ & Bark & Piles & General & Prasad et al. 2008 \\
\hline $\begin{array}{l}\text { Hexalobus monopetalus (A. Rich.) Engl. } \\
\text { and Diels }\end{array}$ & $\begin{array}{l}\text { Shrub or } \\
\text { small tree }\end{array}$ & Leaves & Vegetable & General & Dansi et al. 2008 \\
\hline Miliusa eriocarpa Dunn & $\begin{array}{l}\text { Shrub or } \\
\text { small tree }\end{array}$ & Leaves & Skin diseases; snakebite & General & Dey and De 2012 \\
\hline Miliusa horsfieldii (Bennett) Baill. ex Pierre & $\begin{array}{l}\text { Shrub or } \\
\text { small tree }\end{array}$ & Leaves & Wounds & General & Prasad et al. 2008 \\
\hline $\begin{array}{l}\text { Miliusa montana Gardner ex Hook. f. and } \\
\text { Thomson }\end{array}$ & $\begin{array}{l}\text { Shrub or } \\
\text { small tree }\end{array}$ & Leaves & Fever & General & $\begin{array}{l}\text { Muralidhara Rao and Pullaiah } \\
2007\end{array}$ \\
\hline Polyalthia cerasoides (Roxb.) Bedd. & Tree & Bark, fruit & Bone fractures; edible; to start mensturation & $\begin{array}{l}\begin{array}{l}\text { General; } \\
\text { women }\end{array} \\
\end{array}$ & Ratnam and Raju 2008 \\
\hline Polyalthia longifolia (Sonn.) Thwaites & Tree & $\begin{array}{l}\text { Flower, } \\
\text { leaves, bark }\end{array}$ & Loose motion; menorrhagia; antibacterial & $\begin{array}{l}\text { General; } \\
\text { women }\end{array}$ & Bosco and Arumugam 2012 \\
\hline Sageraea laurifolia (Graham) Blatt. & Tree & Leaves & Rheumatism & General & Kshirsagar and Saklani 2007 \\
\hline Uvaria chamae P. Beauv. & $\begin{array}{l}\text { Shrub or } \\
\text { small tree }\end{array}$ & Leaves & Vegetable & General & Dansi et al. 2008 \\
\hline \multicolumn{6}{|l|}{ Apiaceae } \\
\hline Ammi visnaga (L.) Lam. & $\begin{array}{l}\text { Biennial } \\
\text { herb }\end{array}$ & Fruit & Asthma & General & Kayani et al. 2014 \\
\hline Anethum graveolens $\mathrm{L}$. & Herb & Seed & Cold; cough; mouth sore(cattle) & General & Jain et al. 2004 \\
\hline Angelica glauca Edgew. & $\begin{array}{l}\text { Perennial } \\
\text { herb }\end{array}$ & Root & Joint pains & $\begin{array}{l}\text { General; old } \\
\text { aged }\end{array}$ & Chopda and Mahajan 2009 \\
\hline Centella asiatica (L.) Urb. & $\begin{array}{l}\text { Perennial } \\
\text { herb }\end{array}$ & Whole plant & $\begin{array}{l}\text { Fever; leprosy; dysentery; inflammation; jaundice; } \\
\text { headache and memory power; neurosis; skin disease; } \\
\text { syphiilis; nervine tonic; voice clarity; cough; body; } \\
\text { refregirant; tooth ache; antibacterial; blood pressure; } \\
\text { memory power; leprosy; cardiac disorders; fish poison; } \\
\text { veneral diseases }\end{array}$ & $\begin{array}{l}\text { General; } \\
\text { women; } \\
\text { children }\end{array}$ & $\begin{array}{l}\text { Krishna et al. 2011, } \\
\text { Pushpangadan and Atal } 1984\end{array}$ \\
\hline
\end{tabular}

Published 11 February 2019

http://dx.doi.org/10.32859/era.18.4.1-112 


\begin{tabular}{|c|c|c|c|c|c|}
\hline Coriandrum sativum L. & Herb & Whole plant & $\begin{array}{l}\text { Lack of appetite; intestinal gas; weakness; inflammation; } \\
\text { joint pain; gum disease; scurvy; scabies; indigrstion; piles; } \\
\text { throat pain; asthma; cough; bronchitis; lactation }\end{array}$ & $\begin{array}{l}\text { General; } \\
\text { women }\end{array}$ & $\begin{array}{l}\text { Kayani et al. 2014, Vedavathy et } \\
\text { al. } 1997\end{array}$ \\
\hline Cuminum cyminum L. & Herb & Seed, fruit & $\begin{array}{l}\text { Diarrhea; dysentery; sore throat; loss of appetite; } \\
\text { gonohrrea; contraceptive; menorrhegia; antidiabetic; } \\
\text { hyperacidity; ear sore; dry cough; cold; increase lactation; } \\
\text { jaundice }\end{array}$ & $\begin{array}{l}\text { Women; } \\
\text { men }\end{array}$ & $\begin{array}{l}\text { Bhat et al. 2014, Eneh et al. } \\
\text { 2013, Kayani et al. 2014, } \\
\text { Lingaraju et al. } 2013\end{array}$ \\
\hline Daucus carota L. & $\begin{array}{l}\text { Biennial } \\
\text { herb }\end{array}$ & Seed & Regularises menstruation; arborifacient; amenorrhoea & $\begin{array}{l}\text { Women; } \\
\text { pregnant } \\
\text { women }\end{array}$ & Jain et al. 2004, Patel 2010 \\
\hline Eryngium foetidum $\mathrm{L}$. & $\begin{array}{l}\text { Perennial } \\
\text { herb }\end{array}$ & Whole plant & Diarrhoea; stomachic; antiseptic; flavouring agent & General & Lingaraju et al. 2013 \\
\hline Ferula assa-foetida L. & Herb & $\begin{array}{l}\text { Gum, root, } \\
\text { latex }\end{array}$ & Asthma; cough; contraceptive & General & Kayani et al. 2014 \\
\hline Ferula jaeschkeana Vatke & Herb & Gum & - & - & Chopda and Mahajan 2009 \\
\hline Ferula narthex Boiss. & Herb & Whole plant & Cough; asthma; whooping cough; pneumonia; bronchitis & General & $\begin{array}{l}\text { Kayani et al. 2014, Sharma et al } \\
2013\end{array}$ \\
\hline Foeniculum vulgare Mill. & Herb & Aerial parts & $\begin{array}{l}\text { To eliminate gases; for bellyache; laxative; to urinate; for } \\
\text { bad breath; refreshing; increases lactation }\end{array}$ & $\begin{array}{l}\text { General; } \\
\text { women }\end{array}$ & Parada et al. 2009 \\
\hline Heracleum rigens Wall. ex DC. & $\begin{array}{l}\text { Perennial } \\
\text { herb }\end{array}$ & Root, fruit & Diuretics; asthma; antiseptic & General & Lingaraju et al. 2013 \\
\hline Peucedanum anamallayense C.B. Clarke & $\begin{array}{l}\text { Decumbent } \\
\text { herb }\end{array}$ & Whole plant & Snakebite & General & Udayan et al. 2005 \\
\hline Pimpinella diversifolia DC. & Herb & Whole plant & Cold; cough; digestive disorders & General & Kumar et al. 2011 \\
\hline Pimpinella heyneana (DC.) Benth. & Herb & Root & Diarrhoea; antiseptic & General & Lingaraju et al. 2013 \\
\hline $\begin{array}{l}\text { Pimpinella tirupatiensis N.P. Balakr. and } \\
\text { Subram. }\end{array}$ & Herb & $\begin{array}{l}\text { Tuber, } \\
\text { leaves }\end{array}$ & Abscess; skin diseases & General & Ram et al. 2004 \\
\hline $\begin{array}{l}\text { Saposhnikovia divaricata (Turcz.) } \\
\text { Schischk. }\end{array}$ & Herb & - & Anti-cancer & General & Zhang et al. 2013 \\
\hline Selinum vaginatum C.B. Clarke & Herb & Root & - & General & Sharma et al. 2013 \\
\hline Trachyspermum ammi (L.) Sprague & Grace & Whole plant & Sore throat; whooping cough; acidity & General & $\begin{array}{l}\text { Jain et al. 2004, Sharma et al. } \\
2013\end{array}$ \\
\hline \multicolumn{6}{|l|}{ Apocynaceae } \\
\hline Aganosma cymosa (Roxb.) G. Don & Climber & Whole plant & As anthelmintic and ophthalmic; boils & General & Sarvalingam et al. 2011 \\
\hline Aganosma dichotoma (Roth) K. Schum. & Climber & Leaves & Fractures & General & Rao et al. 2006 \\
\hline Allamanda cathartica $\mathrm{L}$. & Shurb & Flower & Liver diseases & General & Jain 2004 \\
\hline Alstonia macrophylla Wall. ex G. Don & Tree & $\begin{array}{l}\text { Leaves, } \\
\text { bark, root }\end{array}$ & $\begin{array}{l}\text { Sprains; bruise; dislocated joints; stomach ache; skin } \\
\text { diseases; urinary infections; anti-choleric; vulnerary } \\
\text { properties; fever; aphrodisiac; anticholeric; }\end{array}$ & General & Khyade et al. 2014 \\
\hline Alstonia scholaris (L.) R. Br. & Tree & Whole plant & $\begin{array}{l}\text { swellings; for abortion; Diarrhea; Jaundice and dysentery; } \\
\text { Itching; Menstrual disorder; Post-pregnancy fever; } \\
\text { Rheumatism; skin disease; snake bite; liver complaints }\end{array}$ & $\begin{array}{l}\text { Women; } \\
\text { General }\end{array}$ & $\begin{array}{l}\text { Ayyanar and Ignacimuthu 2005, } \\
\text { Bhat et al. 2012, Khyade et al. } \\
2014\end{array}$ \\
\hline Alstonia venenata $\mathrm{R}$. Br. & Tree & $\begin{array}{l}\text { Leaves, } \\
\text { fruit, stem, } \\
\text { bark }\end{array}$ & $\begin{array}{l}\text { Scabies; snake bite; stored as tablets; antibacterial; } \\
\text { rheumatism }\end{array}$ & General & $\begin{array}{l}\text { Sharma et al. 2013, Valsaraj et } \\
\text { al. } 1997\end{array}$ \\
\hline
\end{tabular}

Published 11 February 2019

http://dx.doi.org/10.32859/era.18.4.1-112 


\begin{tabular}{|c|c|c|c|c|c|}
\hline Anodendron paniculatum A.DC. & $\begin{array}{l}\text { Woodly } \\
\text { climber }\end{array}$ & Whole plant & Fits; Leprosy; Paralysis; bone fracture & General & Prasad et al. 2008 \\
\hline Asclepias curassavica L. & $\begin{array}{l}\text { Perennial } \\
\text { herb }\end{array}$ & $\begin{array}{l}\text { Root, } \\
\text { leaves, } \\
\text { latex, flower }\end{array}$ & $\begin{array}{l}\text { Purgative; astringent; gonorrhea; good styptic; cancer; } \\
\text { Skin diseases; anthelmintic }\end{array}$ & General & Lingaraju et al. 2013 \\
\hline $\begin{array}{l}\text { Brachystelma swarupa K.K. Kumar and } \\
\text { Goyder }\end{array}$ & Herb & Rhizome & Edible & General & Jayakumar et al. 2010 \\
\hline Calotropis gigantea (L.) Dryand. & Herb & $\begin{array}{l}\text { Bark, latex, } \\
\text { root, stem, } \\
\text { flower, } \\
\text { leaves }\end{array}$ & $\begin{array}{l}\text { Elephantiasis; snake bite; thorn induced wound; stomach } \\
\text { ache; dog bite; epilepsy; leucorrhea; antibacterial; laxative; } \\
\text { leprosy; abscess; leprotic wounds; eczema; intestinal } \\
\text { worm; dyspepsia; herpes; pruritus; antiasthmatic; ulcers; } \\
\text { foot problems; skin disease; diaphoretic }\end{array}$ & $\begin{array}{l}\text { Women; } \\
\text { Men; } \\
\text { Children; } \\
\text { General; } \\
\text { cattle }\end{array}$ & $\begin{array}{l}\text { Kumar et al. 2007, Surveswaran } \\
\text { et al. } 2010\end{array}$ \\
\hline Calotropis procera (Aiton) Dryand. & Herb & $\begin{array}{l}\text { Latex, } \\
\text { flower, } \\
\text { leaves, root, } \\
\text { bark, } \\
\text { vegetative } \\
\text { buds }\end{array}$ & $\begin{array}{l}\text { Dysentery; Syphilitic ulceration; Liver enlargement; } \\
\text { Rheumatic joints; ulcers; Oligomenorrhoea; intermittent } \\
\text { fever; epistaxis; Hypertension; Scorpion sting; Stomach } \\
\text { ache; Gastric trouble.; Tumour in stomach; Corn; piles; } \\
\text { Epilepsy; Leprosy; dropsy; Painful gums; intestinal worms; } \\
\text { Dental caries; Cholera; jaundice; gastritis; snake bite; } \\
\text { epilepsy; abortion; tuberculosis; }\end{array}$ & General & $\begin{array}{l}\text { Chopda and Mahajan 2009, Jain } \\
\text { et al. } 2009, \text { Rao et al. 2006, } \\
\text { Sharma et al. 2013, Upadhya et } \\
\text { al. } 2012\end{array}$ \\
\hline Caralluma adscendens (Roxb.) R. Br. & Herb & Whole plant & Obesity; skin diseases & General & Jayanthi et al. 2012 \\
\hline $\begin{array}{l}\text { Caralluma bicolor V.S. Ramach., S. Jose, } \\
\text { H.A. John and Sofiya }\end{array}$ & Herb & Whole plant & Wound healing; weight loss & General & Dhivya and Kalaichelvi 2015 \\
\hline Caralluma diffusa (Wight) N.E. Br. & Herb & Whole plant & Obesity & General & Jayanthi et al. 2012 \\
\hline Caralluma umbellata Haw. & Herb & Whole plant & Epilepsy; indigestion; constipation; burns & General & $\begin{array}{l}\text { Kalaiselvan and Gopalan 2014, } \\
\text { Sharma et al. 2013, Sreeramulu } \\
\text { et al. } 2013\end{array}$ \\
\hline Carissa carandas $\mathrm{L}$. & Shrub & $\begin{array}{l}\text { Leaves, } \\
\text { root, fruit, } \\
\text { bark }\end{array}$ & Antihelmitic; wound healing; diabetes; fever; edible & General & Jain et al. 2009 \\
\hline Carissa spinarum $\mathrm{L}$. & Shrub & Whole plant & Wound healing; rheumatism; skin disease; asthma & General & $\begin{array}{l}\text { Kayani et al. 2014, Sharma et al. } \\
2013\end{array}$ \\
\hline Cascabela thevetia (L.) Lippold & Shrub & Seed, fruit & Treat veneral disease; abortion & $\begin{array}{l}\text { General; } \\
\text { pregnant } \\
\text { women }\end{array}$ & Kanthasamy Kalaichelvi 2016 \\
\hline Catharanthus pusillus (Murray) G. Don & Herb & Whole plant & $\begin{array}{l}\text { Rheumatic pain and inflammation of joints; against } \\
\text { lumbago and sciatica. }\end{array}$ & General & - \\
\hline Catharanthus roseus (L.) G. Don & Herb & Whole plant & $\begin{array}{l}\text { To treat malaria and malaria-like symptoms; antidiabetic; } \\
\text { blood pressure; cancer; loose motion; hypertension; } \\
\text { tuberculosis; snake bite }\end{array}$ & General & $\begin{array}{l}\text { Chenniappan and Kadarkarai } \\
\text { 2010, Kadhirvel et al. 2010, } \\
\text { Lingaraju et al. } 2013\end{array}$ \\
\hline Ceropegia bulbosa Roxb. & Herb & Bulb, leaves & Urinary disorder; Dysentery; diarrhoea & General & Jain et al. 2004 \\
\hline Ceropegia candelabrum L. & Herb & $\begin{array}{l}\text { Leaves, } \\
\text { stem, tuber, } \\
\text { gum }\end{array}$ & $\begin{array}{l}\text { To cure one-sided headache; to promote fertility and } \\
\text { vitality; urinary disorders }\end{array}$ & $\begin{array}{l}\text { General; } \\
\text { women }\end{array}$ & $\begin{array}{l}\text { Ayyanar and Ignacimuthu } 2005 \text {, } \\
\text { Jain et al. } 2004\end{array}$ \\
\hline Ceropegia juncea Roxb. & Herb & Stem, tuber & Stomach ulcer; Galactogogue & General & Sarvalingam et al. 2011 \\
\hline Chilocarpus denudatus Blume & Climber & Leaves & Psoriasis & General & Prasad et al. 2013 \\
\hline
\end{tabular}

Published 11 February 2019

http://dx.doi.org/10.32859/era.18.4.1-112 


\begin{tabular}{|c|c|c|c|c|c|}
\hline Chonemorpha fragrans (Moon) Alston & Shurb & Leaves & Psoriasis; Scabies & General & Prasad et al. 2013 \\
\hline Cryptolepis dubia (Burm. f.) M.R. Almeida & $\begin{array}{l}\text { Climbing } \\
\text { shrub }\end{array}$ & Whole plant & $\begin{array}{l}\text { Fissure on sole; fever; rheumatic pain; dysentery; dropsy; } \\
\text { cuts; cholera; abdominal pain; body ache; stomach ache; } \\
\text { syphilis; stop bleeding; eczema; scabies; increase } \\
\text { lactation; herpes; snakebite; tooth troubles; skin diseases; } \\
\text { galactogogue }\end{array}$ & $\begin{array}{l}\text { General, } \\
\text { cattle }\end{array}$ & $\begin{array}{l}\text { Bhat et al. 2014, Ignacimuthu et } \\
\text { al. 2008, Ramya } 2008\end{array}$ \\
\hline Cryptostegia grandiflora Roxb. ex R. Br. & $\begin{array}{l}\text { Climbing } \\
\text { shrub }\end{array}$ & Whole plant & Gastrointestinal disorders; wounds & General & Rao et al. 2006 \\
\hline $\begin{array}{l}\text { Cynanchum paniculatum (Bunge) Kitag. } \\
\text { ex H. Hara }\end{array}$ & $\begin{array}{l}\text { Perennial } \\
\text { climbing } \\
\text { herb }\end{array}$ & - & Anti-proliferative & General & Zhang et al. 2013 \\
\hline $\begin{array}{l}\text { Decalepis arayalpathra (J. Joseph and V. } \\
\text { Chandras.) Venter }\end{array}$ & Shrub & - & $\begin{array}{l}\text { Anti tumour; remedy for peptic ulcer; anticancer; } \\
\text { rejuvenating tonic }\end{array}$ & General & Pushpagadan et al. 1990 \\
\hline Decalepis hamiltonii Wight and Arn. & Shrub & Root, fruit & Cooling agent; hair wash & General & Rao et al. 2006 \\
\hline Dregea volubilis (L.f.) Benth. ex Hook. f. & $\begin{array}{l}\text { Tree/ Shrub/ } \\
\text { Climber/ } \\
\text { Herb-Woody } \\
\text { Climber }\end{array}$ & Whole plant & $\begin{array}{l}\text { Paralysis; rheumatism; tonsils; neck pain; antidiabetic; } \\
\text { boils; diabetes; snakebite; dyspepsia; rheumatic pain; } \\
\text { dysentery; cough; fever; urinary troubles; swellings(cattle) }\end{array}$ & $\begin{array}{l}\text { General; } \\
\text { cattle }\end{array}$ & $\begin{array}{l}\text { Kumar et al. 2007, Pradheeps } \\
\text { and Poyyamoli } 2013 \text {, } \\
\text { Sreeramulu et al. } 2013\end{array}$ \\
\hline Gymnema hirsutum Wall. & $\begin{array}{l}\text { Twining } \\
\text { shrub }\end{array}$ & Leaves & Antidiabetic; jaundice & General & Manikandan et al. 2006 \\
\hline Gymnema sylvestre (Retz.) R. Br. ex Sm. & Herb & Whole plant & $\begin{array}{l}\text { Gas trouble; diabetes; asthma; poison bites; skin } \\
\text { diseases; fever; galactogogue; joint pains; breathing } \\
\text { trouble; constipation; jaundice; snake and scorpion bites; } \\
\text { irregular periods; antiseptic; boils; blisters }\end{array}$ & $\begin{array}{l}\text { General; } \\
\text { women }\end{array}$ & $\begin{array}{l}\text { Manikandan et al. 2006, Ramya } \\
\text { 2008, Samy et al. 2008, } \\
\text { Sreeramulu et al. } 2013\end{array}$ \\
\hline Hemidesmus indicus (L.) R. Br. ex Schult. & Herb & Whole plant & $\begin{array}{l}\text { Purifies blood; snake bite; body heat; increases semen } \\
\text { production; antipyretic; diarrhoea; rheumatism; ulcer; } \\
\text { syphilis; leucorrhea; respiratory and urinary disorders; } \\
\text { cough; asthma; colostrum; male reproductive disorder; } \\
\text { piles; skin disorder; herpes; gynaecological disorders; } \\
\text { convulsive seizure; antibacterial }\end{array}$ & $\begin{array}{l}\text { General; } \\
\text { men; } \\
\text { women }\end{array}$ & $\begin{array}{l}\text { Ayyanar } 2013 \text {, Dey and De } \\
2012, \text { Ignacimuthu et al. } 2008, \\
\text { Mahishi et al. } 2005, \\
\text { Pushpangadan and Atal } 1984, \\
\text { Ramya } 2008\end{array}$ \\
\hline $\begin{array}{l}\text { Himatanthus obovatus (Müll. Arg.) } \\
\text { Woodson }\end{array}$ & Tree & - & Antifungal & General & Rodrigues and Carlini 2005 \\
\hline Holarrhena pubescens Wall. ex G. Don & Shrub & $\begin{array}{l}\text { Bark, } \\
\text { leaves, } \\
\text { seed, root, } \\
\text { flower, latex }\end{array}$ & $\begin{array}{l}\text { Eczema; pruritus; snakebite; diarrhoea; dysentery; } \\
\text { leucoderma; anthelmintic; cough; headache; sprains; skin } \\
\text { diseases; intestinal worms; body pains; arthritis; increases } \\
\text { lactation; malaria; amenorrhegia; cuts; wound; } \\
\text { antibacterial }\end{array}$ & $\begin{array}{l}\text { General; } \\
\text { women }\end{array}$ & $\begin{array}{l}\text { Dey and De 2012, Jain 2004, } \\
\text { Rao et al. 2006, Valsaraj et al. } \\
1997\end{array}$ \\
\hline Holostemma ada-kodien Schult. & Climber & Latex & $\begin{array}{l}\text { Eye diseases; postpartum health; tuberculosis; eye } \\
\text { diseases; burns }\end{array}$ & General & $\begin{array}{l}\text { Chithra et al. 2016, Rao et al. } \\
2006\end{array}$ \\
\hline Hoya parasitica Wall. ex Traill & Shrub & Leaves & Bone fracture & General & Chander et al. 2015 \\
\hline Hoya pendula Wight and Arn. & Climber & Leaves, root & Eye infection; prolapse of uterus; heart pain & $\begin{array}{l}\text { General; } \\
\text { women }\end{array}$ & Rao et al. 2011 \\
\hline Ichnocarpus frutescens (L.) W.T. Aiton & Climber & Whole plant & $\begin{array}{l}\text { Rheumatism; blood purifier; asthma; cough; bronchitis; } \\
\text { bone fracture; cholera; constipation; dysentery; fever; nigh } \\
\text { blindness; measles; ulcer; vomiting tonic; febrifuge; }\end{array}$ & General & $\begin{array}{l}\text { Adhikari et al. 2010, Padal et al. } \\
2010 \text {, Rao et al. 2011, Ratnam } \\
\text { and Raju } 2008\end{array}$ \\
\hline
\end{tabular}

Published 11 February 2019

http://dx.doi.org/10.32859/era.18.4.1-112 


\begin{tabular}{|c|c|c|c|c|c|}
\hline & & & $\begin{array}{l}\text { leucoderma; diabetes; antipyretic; stomach cancer; skin } \\
\text { diseases; hemorrhage; galactagogue; respiratory disorder }\end{array}$ & & \\
\hline $\begin{array}{l}\text { Leptadenia lancifolia (Schumach. and } \\
\text { Thonn.) Decne. }\end{array}$ & $\begin{array}{l}\text { Perennial } \\
\text { climber }\end{array}$ & Leaves & Vegetable & General & Dansi et al. 2008 \\
\hline Leptadenia pyrotechnica (Forssk.) Decne. & $\begin{array}{l}\text { Decidious } \\
\text { shrub }\end{array}$ & $\begin{array}{l}\text { Stem, } \\
\text { leaves }\end{array}$ & Tuberculosis; wound; expulsion of placenta & $\begin{array}{l}\text { General; } \\
\text { women }\end{array}$ & Dhanabal et al. 2015 \\
\hline $\begin{array}{l}\text { Leptadenia reticulata (Retz.) Wight and } \\
\text { Arn. }\end{array}$ & Climber & Whole plant & $\begin{array}{l}\text { Aphrodisiac; restorative; febrifuge; hairwash; earache; } \\
\text { typhoid; skin disease; asthma; eczema }\end{array}$ & General & $\begin{array}{l}\text { Padal et al. 2010, Rao et al. } \\
2006\end{array}$ \\
\hline Marsdenia tenacissima (Roxb.) Moon & Climber & Root & Snake bite & General & Sreeramulu et al. 2013 \\
\hline Nerium oleander $\mathrm{L}$. & Shrub & Whole plant & $\begin{array}{l}\text { Antidote; ulcer; leprosy; piles; skin diseases; } \\
\text { gastrointestinal disorders; for speech to children; } \\
\text { snakebite; earache; dysentery }\end{array}$ & General & $\begin{array}{l}\text { Chopda and Mahajan 2009, Dey } \\
\text { and De } 2012\end{array}$ \\
\hline Oxystelma esculentum (L.f.) Sm. & Climber & Leaves & Gonorrhea; cough; joint pain; tooth ache; jaundice & General & $\begin{array}{l}\text { Kanthasamy Kalaichelvi } 2016 \text {, } \\
\text { Rao et al. } 2006\end{array}$ \\
\hline Pergularia daemia (Forssk.) Chiov. & Climber & Whole plant & $\begin{array}{l}\text { Abortion; gonorrhea; acidity; asthma; scorpion bite; fits; } \\
\text { rheumatism; piles; boils; oedema; menstural troubles; } \\
\text { insect bites; headache; liver diseases; jaundice; laxative; } \\
\text { antipyretic; urinary disorders; chest pain; stomach ulcers; } \\
\text { antidiabetic; leprotic wounds; snakebite; cough }\end{array}$ & $\begin{array}{l}\text { General; } \\
\text { women; } \\
\text { pregnant } \\
\text { women }\end{array}$ & $\begin{array}{l}\text { Hebbar et al. 2004, Ignacimuthu } \\
\text { et al. 2008, Kosalge and Fursule } \\
2009 \text {, Kshirsagar and Singh } \\
2001\end{array}$ \\
\hline Plumeria rubra L. & Tree & $\begin{array}{l}\text { Bark, latex, } \\
\text { root, flower }\end{array}$ & $\begin{array}{l}\text { Eczema; scabies; menstural pains; veneral diseases; } \\
\text { aphrodisiac; mouth ulcer; antiinflammation }\end{array}$ & General & Vijaykumar and Taranath 2010 \\
\hline Rauvolfia serpentina (L.) Benth. ex Kurz & Herb & $\begin{array}{l}\text { Root, leaves } \\
\text { bud, } \\
\text { rhizome, } \\
\text { leaves }\end{array}$ & $\begin{array}{l}\text { Abortifacient; epilepsy; herpes; pruritus; scabies; malaria; } \\
\text { nervous disorders; snakebite; stomach pain; chest pain; } \\
\text { hypertension; blood pressure }\end{array}$ & $\begin{array}{l}\text { General; } \\
\text { pregnant } \\
\text { women }\end{array}$ & $\begin{array}{l}\text { Chopda and Mahajan 2009, } \\
\text { Lingaraju et al. 2013, } \\
\text { Pushpangadan and Atal } 1984\end{array}$ \\
\hline Rauvolfia tetraphylla L. & Shrub & Seed, root & Anti snake venom; skin diseases & General & Dey and De 2012 \\
\hline Rhazya stricta Decne. & Shrub & - & Snakebite & General & Dey and De 2012 \\
\hline Sarcostemma acidum (Roxb.) Voigt & Shrub & Bark, root & Galactogogue; snake bite; bone fracture; earache & General & $\begin{array}{l}\text { Rao et al. 2006, Sreeramulu et } \\
\text { al. } 2013\end{array}$ \\
\hline Sarcostemma brevistigma Wight and Arn. & Climber & Aerial parts & Asthma; antioxidant & General & Kanthasamy Kalaichelvi 2016 \\
\hline Sarcostemma intermedium Decne. & Climber & Stem, latex & Body swelling; snakebite & General & Kosalge and Fursule 2009 \\
\hline Sarcostemma secamone (L.) Bennett & Climber & Latex & Galactogogue & General & Sreeramulu et al. 2013 \\
\hline Sarcostemma viminale (L.) R. Br. & Climber & Latex & Snake bite & General & Sarvalingam et al. 2011 \\
\hline $\begin{array}{l}\text { Secamone afzelii (Roem. and Schult.) K. } \\
\text { Schum. }\end{array}$ & Climber & Leaves & Vegetable & General & Dansi et al. 2008 \\
\hline $\begin{array}{l}\text { Secamone emetica (Retz.) R. Br. ex } \\
\text { Schult. }\end{array}$ & Climber & Leaves & Nervous disorder; joint pains & General & Sarvalingam et al. 2011 \\
\hline Tabernaemontana alternifolia L. & Tree & $\begin{array}{l}\text { Latex, } \\
\text { leaves, root, } \\
\text { bark, stem }\end{array}$ & $\begin{array}{l}\text { Eczema; diabetes; snake bite; skin diseases; prolonged } \\
\text { labour; herpes; pruritus; scabies; skin sore; abortion }\end{array}$ & $\begin{array}{l}\text { General; } \\
\text { women }\end{array}$ & $\begin{array}{l}\text { Harsha et al. 2003, Lingaraju et } \\
\text { al. } 2013\end{array}$ \\
\hline $\begin{array}{l}\text { Tabernaemontana dichotoma Roxb. ex } \\
\text { Wall. }\end{array}$ & Shrub/ Tree & Leaves & Tooth ache & General & Deepa et al. 2011 \\
\hline $\begin{array}{l}\text { Tabernaemontana divaricata (L.) R. Br. ex } \\
\text { Roem. and Schult. }\end{array}$ & Shrub & $\begin{array}{l}\text { Root, bark, } \\
\text { rhizome, }\end{array}$ & $\begin{array}{l}\text { Snakebite; skin eruption; antiseptic; amoebic dysentery; } \\
\text { fish poisoning; eye diseases; boils; tooth decay }\end{array}$ & General & $\begin{array}{l}\text { Dey and De 2012, Lingaraju et } \\
\text { al. } 2013\end{array}$ \\
\hline
\end{tabular}

Published 11 February 2019

http://dx.doi.org/10.32859/era.18.4.1-112 
Ethnobotany Research and Applications

\begin{tabular}{|c|c|c|c|c|c|}
\hline & & $\begin{array}{l}\text { leaves, } \\
\text { flower, latex }\end{array}$ & & & \\
\hline Thevetia neriifolia Juss. ex Steud. & Tree & Bark & Skin diseases & General & Harsha et al. 2003 \\
\hline Tylophora asthmatica (L.f.) Wight and Arn. & $\begin{array}{l}\text { Climbing } \\
\text { shrub }\end{array}$ & Leaves & Asthma; snakebite; jaundice & General & Mahishi et al. 2005 \\
\hline $\begin{array}{l}\text { Tylophora fasciculata Buch. -Ham. ex } \\
\text { Wight }\end{array}$ & Herb & Leaves & Wound & General & Chopda and Mahajan 2009 \\
\hline Tylophora flexuosa R. Br. & $\begin{array}{l}\text { Climbing } \\
\text { shrub }\end{array}$ & Stem & Repel leeches & General & Nizar et al. 2015 \\
\hline Tylophora indica (Burm. f.) Merr. & $\begin{array}{l}\text { Climbing } \\
\text { shrub }\end{array}$ & $\begin{array}{l}\text { Root, } \\
\text { leaves, stem }\end{array}$ & $\begin{array}{l}\text { Urinary problems; snakebite; asthma; lactation; boils; } \\
\text { stomach troubles; insect bite }\end{array}$ & $\begin{array}{l}\text { General; } \\
\text { women }\end{array}$ & $\begin{array}{l}\text { Dey and De 2012, Padma et al. } \\
\text { 2016, Sarvalingam et al. } 2011\end{array}$ \\
\hline Tylophora longifolia Wight & Shrub & $\begin{array}{l}\text { Leaves, } \\
\text { flower }\end{array}$ & Snakebite & General & Samy et al. 2008 \\
\hline $\begin{array}{l}\text { Tylophora rotundifolia Buch. -Ham. ex } \\
\text { Wight }\end{array}$ & Climber & Leaves & Kidney disorder & General & Wagh and Jain 2015 \\
\hline Utleria salicifolia Bedd. ex Hook. f. & Shrub & Rhizome & Antiulcer activity & General & Rao et al. 2004 \\
\hline Vallaris solanacea (Roth) Kuntze & $\begin{array}{l}\text { Climbing } \\
\text { shrub }\end{array}$ & Latex, stem & Sores; syphilis; abortificient; wounds & $\begin{array}{l}\text { General; } \\
\text { pregnant } \\
\text { women }\end{array}$ & Adhikari et al. 2010 \\
\hline Willughbeia edulis Roxb. & Climber & Leaves & - & General & Dey and De 2012 \\
\hline Wrightia arborea (Dennst.) Mabb. & Tree & $\begin{array}{l}\text { Bark, latex, } \\
\text { leaves }\end{array}$ & Skin diseases; snake bite; urinary stones; menstural pain & $\begin{array}{l}\text { General; } \\
\text { women }\end{array}$ & Prasad et al. 2008 \\
\hline Wrightia tinctoria R. Br. & Tree & $\begin{array}{l}\text { Seed, } \\
\text { leaves, fruit, } \\
\text { latex, root }\end{array}$ & $\begin{array}{l}\text { Boils; blisters; wounds; fever; aphrodisiac; dandruff; } \\
\text { headache; for easy delivery; mouth ulcers; epilepsy; } \\
\text { psoriasis; skin diseases; sore throat; cough; toothache }\end{array}$ & $\begin{array}{l}\text { General; } \\
\text { women; } \\
\text { pregnant } \\
\text { women }\end{array}$ & $\begin{array}{l}\text { Jain et al. 2004, Udayan et al. } \\
2005\end{array}$ \\
\hline \multicolumn{6}{|l|}{ Aponogetonaceae } \\
\hline Aponogeton appendiculatus $\mathrm{H}$. Bruggen & Herb & Tuber & Edible & General & Narayanan et al. 2011 \\
\hline \multicolumn{6}{|l|}{ Araceae } \\
\hline Alocasia macrorrhizos (L.) G. Don & Herb & Leaves & Piles & General & Chander et al. 2015 \\
\hline Amorphophallus bulbifer (Roxb.) Blume & Herb & $\begin{array}{l}\text { Rhizome, } \\
\text { bulbils, } \\
\text { petiole }\end{array}$ & $\begin{array}{l}\text { Piles and gonorrhea; hemorrhoids and diarrhea; } \\
\text { rheumatism; animal bites }\end{array}$ & General & Reddy et al. 2012 \\
\hline $\begin{array}{l}\text { Amorphophallus paeoniifolius (Dennst.) } \\
\text { Nicolson }\end{array}$ & Herb & Tuber, corm & $\begin{array}{l}\text { Tumors; used for post-delivery problems; Bleeding piles; } \\
\text { bronchitis and asthma; bone fracture; joint disease }\end{array}$ & $\begin{array}{l}\text { Women; } \\
\text { General }\end{array}$ & $\begin{array}{l}\text { Bhuvaneswari et al. } 2015 \text {, } \\
\text { Upadhya et al. } 2012\end{array}$ \\
\hline Amorphophallus sylvaticus (Roxb.) Kunth & Herb & Tuber & Cough; sexual weakness & General & Bhuvaneswari et al. 2015 \\
\hline Anaphyllum beddomei Engl. & Herb & $\begin{array}{l}\text { Rhizome, } \\
\text { leaves }\end{array}$ & Eczema; scabies; food; anti snake venom & General & Sulochana et al. 2015 \\
\hline Anaphyllum wightii Schott & Herb & $\begin{array}{l}\text { Tuber, root, } \\
\text { corms }\end{array}$ & Snakebite & General & Udayan et al. 2007 \\
\hline Arisaema flavum (Forssk.) Schott & $\begin{array}{l}\text { Perennial } \\
\text { herb }\end{array}$ & Rhizome & Scorpion stings & General & Sharma et al. 2013 \\
\hline Arisaema leschenaultii Blume & Herb & Tuber, fruit & Antiseptic; snake bite & General & Pradheeps and Poyyamoli 2013 \\
\hline Arisaema tortuosum (Wall.) Schott & Herb & $\begin{array}{l}\text { Corm, tuber, } \\
\text { root }\end{array}$ & $\begin{array}{l}\text { Taken internally against worms and diarrhoea; snake bite; } \\
\text { boils }\end{array}$ & $\begin{array}{l}\text { General; } \\
\text { cattle }\end{array}$ & Swarnkar and Katewa 2008 \\
\hline
\end{tabular}

Published 11 February 2019

http://dx.doi.org/10.32859/era.18.4.1-112 


\begin{tabular}{|c|c|c|c|c|c|}
\hline Arum italicum Mill. & Herb & Fruit & For piles & General & Parada et al. 2009 \\
\hline Colocasia esculenta (L.) Schott & Herb & $\begin{array}{l}\text { Leaves, } \\
\text { rhizome, } \\
\text { corm, } \\
\text { petiole, } \\
\text { tuber }\end{array}$ & $\begin{array}{l}\text { Urinary tract problems; boils; edible; kidney stones; } \\
\text { General debility; vegetable; piles; stomach disorders and } \\
\text { indigestion; stomach ulcers }\end{array}$ & General & $\begin{array}{l}\text { Dansi et al. 2008, Rajendran et } \\
\text { al. } 2000\end{array}$ \\
\hline $\begin{array}{l}\text { Epipremnum aureum (Linden and André) } \\
\text { G.S. Bunting }\end{array}$ & Herb & Leaves & Gastric problems; gastric ulcer & General & Rahmatullah et al. 2010 \\
\hline Lasia spinosa (L.) Thwaites & $\begin{array}{l}\text { Perennial } \\
\text { herb }\end{array}$ & $\begin{array}{l}\text { Leaves, } \\
\text { rhizome, } \\
\text { root, fruit }\end{array}$ & $\begin{array}{l}\text { Piles; colic; rheumatism and intestinal diseases; } \\
\text { vegetable; throat infections; snake bite }\end{array}$ & General & Alam et al. 2012 \\
\hline Pinellia ternata (Thunb.) Makino & Herb & - & Anti-tumour & General & Zhang et al. 2013 \\
\hline Pothos chinensis (Raf.) Merr. & Herb & Whole plant & Treating cough or cold & General & Srithi et al. 2009 \\
\hline Pothos scandens L. & Climber & Whole plant & $\begin{array}{l}\text { Delivery wounds; skin allergy; herpes; abscesses; } \\
\text { diarrhoea; muscle catch; cancer; small pox; sprains; bone } \\
\text { fracture; asthma }\end{array}$ & $\begin{array}{l}\text { General; } \\
\text { women }\end{array}$ & $\begin{array}{l}\text { Ayyanar and Ignacimuthu 2005, } \\
\text { Lingaraju et al. } 2013\end{array}$ \\
\hline Remusatia vivipara (Roxb.) Schott & Herb & $\begin{array}{l}\text { Leaves, } \\
\text { tuber }\end{array}$ & Asthma; boils & General & Wagh and Jain 2015 \\
\hline Rhaphidophora decursiva (Roxb.) Schott & Herb & Whole plant & Dysentery; diarrhea; mumps; swelling in growing joints & General & $\begin{array}{l}\text { Pradheeps and Poyyamoli 2013, } \\
\text { Prasad et al. } 2013\end{array}$ \\
\hline Rhaphidophora pertusa (Roxb.) Schott & Climber & $\begin{array}{l}\text { Stem, root, } \\
\text { leaves, fruit }\end{array}$ & $\begin{array}{l}\text { Earache; treating liver disorders; to treat enlarged liver and } \\
\text { spleen; skin diseases; for bandaging the plastered } \\
\text { joints/broken bones; cuts and wounds; tonic; antidode for } \\
\text { bite of Russell's Viper }\end{array}$ & General & Asha and Pushpagadan 1998 \\
\hline $\begin{array}{l}\text { Sauromatum venosum (Dryand. ex Aiton) } \\
\text { Kunth }\end{array}$ & Herb & Whole plant & Anthelmintic; stimulating poultice & General & $\begin{array}{l}\text { Dey and De 2012, Wagh and } \\
\text { Jain } 2015\end{array}$ \\
\hline Stylochaeton Iancifolius Kotschy and Peyr. & Herb & Leaves & Vegetable & General & Dansi et al. 2008 \\
\hline \multicolumn{6}{|l|}{ Araliaceae } \\
\hline Hydrocotyle javanica Thunb. & Creeper & Whole plant & Dysentery; menorrhagia; fish poisoning; loose motion & $\begin{array}{l}\text { General; } \\
\text { children; } \\
\text { women }\end{array}$ & Lingaraju et al. 2013 \\
\hline Hydrocotyle sibthorpioides Lam. & $\begin{array}{l}\text { Perennial } \\
\text { herb }\end{array}$ & Leaves & Treating cough or cold & General & Srithi et al. 2009 \\
\hline Schefflera elliptica (Blume) Harms & Shrub & Leaves & Bone fracture & General & Chander et al. 2015 \\
\hline Schefflera stellata (Gaertn.) Baill. & Tree & $\begin{array}{l}\text { Tender } \\
\text { branches }\end{array}$ & Stomach pain & General & Prabakaran et al. 2013 \\
\hline \multicolumn{6}{|l|}{ Arecaceae } \\
\hline Areca catechu L. & Tree & $\begin{array}{l}\text { Root, seed, } \\
\text { fruit, flower, } \\
\text { pericarp, nut }\end{array}$ & $\begin{array}{l}\text { Oral ulcers; sore mouth; masticatory; wound; jaundice; } \\
\text { herpes; cough; antidiabetic; cuts; fire burn; tooth brush; } \\
\text { Lack of appetite; gum disease; urinary disorders; eye } \\
\text { inflammation; diarrhea; sores; toothache; Anthelmintic; } \\
\text { aphrodisiac; astringent; cooling; digestive; diuretic and } \\
\text { laxative }\end{array}$ & General & $\begin{array}{l}\text { Asha and Pushpangadan 2002, } \\
\text { Mahishi et al. 2005, Manikandan } \\
2005\end{array}$ \\
\hline
\end{tabular}




\begin{tabular}{|c|c|c|c|c|c|}
\hline Arenga wightii Griff. & Tree & Whole plant & $\begin{array}{l}\text { To pregnant ladies to prevent malformed childbirth; } \\
\text { jaundice; to stupefy fish }\end{array}$ & $\begin{array}{l}\text { pregnant } \\
\text { women, } \\
\text { General }\end{array}$ & Asha and Pushpangadan 2002 \\
\hline Borassus flabellifer L. & Tree & $\begin{array}{l}\text { Root, } \\
\text { leaves, root, } \\
\text { rachis, fruit, } \\
\text { petiole }\end{array}$ & $\begin{array}{l}\text { Fertility; contraceptive; non medicinal commercial } \\
\text { purposes; obesity; asthma; eye infection; edible }\end{array}$ & $\begin{array}{l}\text { Women; } \\
\text { General }\end{array}$ & Jain et al. 2004 \\
\hline Calamus rotang $\mathrm{L}$. & Climber & $\begin{array}{l}\text { Tuber, stem, } \\
\text { root, bark, } \\
\text { petiole }\end{array}$ & $\begin{array}{l}\text { Cold; Cough and Fever; non medicinal commercial } \\
\text { purposes; scorpion bite; constipation; boils; wounds }\end{array}$ & General & $\begin{array}{l}\text { Ayyanar and Ignacimuthu 2010, } \\
\text { Bhat et al. } 2012\end{array}$ \\
\hline Calamus tenuis Roxb. & Tree & Fruit, flower & Leucorrhoea; amenorrhoea & General & Adhikari et al. 2010 \\
\hline Calamus thwaitesii Becc. & Climber & Fruit, stem & Burning sensation; gynaecological disorders & $\begin{array}{l}\text { Women; } \\
\text { General }\end{array}$ & $\begin{array}{l}\text { Prashanth Kumar and } \\
\text { Shiddamallayya } 2015\end{array}$ \\
\hline Caryota urens L. & Tree & $\begin{array}{l}\text { Leaves, } \\
\text { toddy, stem, } \\
\text { flower }\end{array}$ & Loss of appetite; body strength; laxative; hydrocele & General & $\begin{array}{l}\text { Ayyanar and Ignacimuthu 2005, } \\
\text { Reddy et al. } 2008\end{array}$ \\
\hline Chamaerops humilis L. & Tree & Stem & Narcotic and digestive & General & $\begin{array}{l}\text { Prashanth Kumar and } \\
\text { Shiddamallayya } 2015\end{array}$ \\
\hline Cocos nucifera L. & Tree & $\begin{array}{l}\text { Fruit, } \\
\text { leaves, oil, } \\
\text { coconut } \\
\text { water }\end{array}$ & $\begin{array}{l}\text { For cordage and matting; non medicinal commercial } \\
\text { purposes; urinary disorders; leucorrhea; skin problems } \\
\text { due to centipede }\end{array}$ & General & $\begin{array}{l}\text { Ayyanar and Ignacimuthu 2010, } \\
\text { Manikandan } 2005\end{array}$ \\
\hline Phoenix loureiroi Kunth & Stout shrub & Leaves, fruit & Dog bite; brooms; edible & General & Kennedy 2006 \\
\hline Phoenix pusilla Gaertn. & Shrub & Fruit & Edible & General & Rekka and Kumar 2014 \\
\hline Phoenix sylvestris (L.) Roxb. & Tree & $\begin{array}{l}\text { Leaves, } \\
\text { root, fruit }\end{array}$ & Toothache; strengthens body & General & Ignacimuthu et al. 2008 \\
\hline \multicolumn{6}{|l|}{ Aristolochiaceae } \\
\hline Aristolochia bracteolata Lam. & Climber & Whole plant & $\begin{array}{l}\text { Irregular menstruation; snake bite; coilc remedy; stomach } \\
\text { pain; skin disease; foul ulcers; gastro intestinal disorders; } \\
\text { insect bite; gonorrhea; cold; Anti-inflammation and } \\
\text { Anthelmintic; amenorrhoea; Fungal infection on head; } \\
\text { eczema; scabies }\end{array}$ & $\begin{array}{l}\text { General; } \\
\text { children; } \\
\text { women }\end{array}$ & $\begin{array}{l}\text { Padal et al. 2014, Reddy et al. } \\
\text { 1991, Rao et al. } 2006\end{array}$ \\
\hline Aristolochia grandiflora Sw. & Climber & Root & Snake bite & General & Das et al. 2013 \\
\hline Aristolochia indica $\mathrm{L}$. & Climber & Whole plant & $\begin{array}{l}\text { Leprosy; snake bite and scorpion bite; skin diseases; } \\
\text { wound; anthelmintic; rash; piles; to prevent snake from } \\
\text { biting; cholera; against worms; diarrhoea; fever; } \\
\text { abotifacient; aphrodisiac; nervoux disorders; tumors }\end{array}$ & General & $\begin{array}{l}\text { Kshirsagar and Singh } 2001 \text {, } \\
\text { Ram et al. 2004, Samy et al. } \\
2008\end{array}$ \\
\hline $\begin{array}{l}\text { Aristolochia krisagathra Sivar. and } \\
\text { Pradeep }\end{array}$ & Climber & $\begin{array}{l}\text { Leaves, } \\
\text { rhizome }\end{array}$ & Snakebite & General & Dey and De 2012 \\
\hline Aristolochia tagala Cham. & Twiner & Whole plant & $\begin{array}{l}\text { Snakebite; to reduce the venom action; to prevent } \\
\text { implantation and to stimulate uterine flow; anti snake } \\
\text { venom; malaria }\end{array}$ & $\begin{array}{l}\text { General; } \\
\text { women }\end{array}$ & $\begin{array}{l}\text { Sarvalingam et al. 2011, Xavier } \\
\text { et al. } 2014\end{array}$ \\
\hline Thottea siliquosa (Lam.) Ding Hou & Shrub & $\begin{array}{l}\text { Root, fruit, } \\
\text { stem bark }\end{array}$ & $\begin{array}{l}\text { Antibacterial; dysentery; cholera; ulcers; diarrhoea; snake } \\
\text { bite; gynaecological disorders; skin diseases; stomach } \\
\text { pain; postpartum health }\end{array}$ & $\begin{array}{l}\text { General; } \\
\text { women }\end{array}$ & $\begin{array}{l}\text { Das et al. 2014, Nizar et al. } \\
2015, \text { Udayan et al. } 2005\end{array}$ \\
\hline
\end{tabular}

Published 11 February 2019

http://dx.doi.org/10.32859/era.18.4.1-112 


\begin{tabular}{|c|c|c|c|c|c|}
\hline \multicolumn{6}{|l|}{ Asparagaceae } \\
\hline Agave americana $\mathrm{L}$. & Shrub & Whole plant & $\begin{array}{l}\text { Tuberculosis; fever; tooth ache; venereal sores and } \\
\text { dysentery; bone dislocation pain }\end{array}$ & General & $\begin{array}{l}\text { Dhanabal et al. 2015, Prashanth } \\
\text { Kumar and Shiddamallayya } \\
2015\end{array}$ \\
\hline Agave vivipara $\mathrm{L}$. & Shrub & - & Fodder plant; Detergent & General & Prabakaran et al. 2013 \\
\hline Agave cantala (Haw.) Roxb. ex Salm-Dyck & Shrub & Leaves, root & Cuts; wounds and burns; snake bite & General & Kumar et al. 2007 \\
\hline Agave vera-cruz Mill. & Shrub & Leaves & Jaundice; liver complaints & General & Mali and Bhadane 2011 \\
\hline Asparagus asiaticus L. & Shrub & Leaves & Swellings. & General & Sarvalingam et al. 2011 \\
\hline Asparagus cochinchinensis (Lour.) Merr. & $\begin{array}{l}\text { Perennial } \\
\text { climber }\end{array}$ & - & Anti-cancer & General & Zhang et al. 2013 \\
\hline Asparagus gonoclados Baker & Climber & Tuber, root & Skin disease & General & Sreeramulu et al. 2013 \\
\hline Asparagus racemosus Willd. & Climber & Whole plant & $\begin{array}{l}\text { To increase lactation; cooling agent; sanke bite; dysentery } \\
\text { and diarrhoea; body ache; wounds; spermatorrhoea; } \\
\text { epilepsy; galacatagogue; foot cracks; jaundice; liver } \\
\text { disease; Hyperacidity; Gastritis; Eye diseases; Diarrhoea; } \\
\text { Urinary diseases; menstrual problems; postpartum health; } \\
\text { kidney problems; skin decay; nervous problems; sores; } \\
\text { typhoid; gynaecological disorders }\end{array}$ & $\begin{array}{l}\text { General; } \\
\text { women }\end{array}$ & $\begin{array}{l}\text { Jain et al. 2004, Rao et al. 2006, } \\
\text { Tetali et al. 2009, Sharma et al. } \\
2013\end{array}$ \\
\hline Chlorophytum arundinaceum Baker & Herb & Root & Impotency & General & Malviya et al. 2011 \\
\hline $\begin{array}{l}\text { Chlorophytum borivilianum Santapau and } \\
\text { R.R. Fern. }\end{array}$ & Herb & Tuber, root & $\begin{array}{l}\text { For physical weakness and health tonic; debility; } \\
\text { anyifertility property }\end{array}$ & General & Wagh and Jain 2015 \\
\hline Chlorophytum tuberosum (Roxb.) Baker & Herb & Leaves & Cold & General & Rao et al. 2006 \\
\hline Dracaena elliptica Thunb. and Dalm. & Shrub & Root & Treating cough or cold & General & Srithi et al. 2009 \\
\hline Dracaena terniflora Roxb. & Shrub & Root, fruit & Spermaturia; jaundice; headache; bed sore & $\begin{array}{l}\text { General; } \\
\text { men }\end{array}$ & Udayan et al. 2005 \\
\hline Drimia indica (Roxb.) Jessop & Herb & $\begin{array}{l}\text { Leaves, } \\
\text { tuber, bulb }\end{array}$ & Boils on legs; acidity; typhoid; fever & General & $\begin{array}{l}\text { Bhuvaneswari et al. 2015, } \\
\text { Puravankara and Gopal 2012, } \\
\text { Sreeramulu et al. } 2013\end{array}$ \\
\hline Furcraea foetida (L.) Haw. & Shrub & Bark & Non medicinal commercial purposes & General & Ayyanar and Ignacimuthu 2010 \\
\hline Ledebouria revoluta (L.f.) Jessop & Herb & Bulb & Urinary problems; body pain; substitute for onion & General & $\begin{array}{l}\text { Ragupathy and Newmaster } \\
2009 \text {, Sreeramulu et al. } 2013\end{array}$ \\
\hline Muscari commutatum Guss. & Herb & Leaves & To repel snakes; antidote to snake bite & General & Dey and De 2012 \\
\hline Ornithogalum umbellatum $\mathrm{L}$. & Herb & Tuber & Stomach pain & General & Bhuvaneswari et al. 2015 \\
\hline $\begin{array}{l}\text { Sansevieria roxburghiana Schult. and } \\
\text { Schult. f. }\end{array}$ & $\begin{array}{l}\text { Stemless } \\
\text { fleshy herb }\end{array}$ & Whole plant & $\begin{array}{l}\text { Snake bite; cardiotonic; expectorant; febrifuge; purgative; } \\
\text { tonic in glandular enlargement; rheumatism; antitumor; } \\
\text { antidiabetic; antibacterial; analgesic; cytotoxic; antioxidant; } \\
\text { ear pain; boils; blisters; jaundice; waist pain; cough }\end{array}$ & General & $\begin{array}{l}\text { Dey and De 2012, Rao et al. } \\
2006 \text {, Seraj et al. 2013, Srithi et } \\
\text { al. } 2013\end{array}$ \\
\hline Sansevieria trifasciata Prain & Herb & Whole plant & Snake repellent; earache; dental caries & General & Dey and De 2012 \\
\hline \multicolumn{6}{|l|}{ Asteraceae } \\
\hline Acanthospermum hispidum DC. & Herb & Whole plant & Skin disease & General & Ram et al. 2004 \\
\hline Achillea millefolium $\mathrm{L}$. & Herb & Whole plant & Cough; Profuse mucous discharges; snake bite & General & $\begin{array}{l}\text { Samy et al. 2008, Sharma et al. } \\
2013\end{array}$ \\
\hline Acmella ciliata (Kunth) Cass. & Herb & Flower & Toothache & General & Udayan et al. 2005 \\
\hline $\begin{array}{l}\text { Acmella paniculata (Wall. ex DC.) R.K. } \\
\text { Jansen }\end{array}$ & Herb & Whole plant & Toothache; mouth ulcers; bleeding wound & General & Ignacimuthu et al. 2008 \\
\hline
\end{tabular}

Published 11 February 2019

http://dx.doi.org/10.32859/era.18.4.1-112 


\begin{tabular}{|c|c|c|c|c|c|}
\hline $\begin{array}{l}\text { Ageratina adenophora (Spreng.) R.M. } \\
\text { King and H. Rob. }\end{array}$ & Shrub & Whole plant & $\begin{array}{l}\text { Body pain; tooth decay; foetus movement; wounds; skin } \\
\text { diseases; cuts }\end{array}$ & $\begin{array}{l}\text { General; } \\
\text { foetus }\end{array}$ & $\begin{array}{l}\text { Kumar et al. 2007, Namsa et al } \\
\text { 2009, Rao et al. } 2011\end{array}$ \\
\hline Ageratum conyzoides (L.) L. & Herb & Whole plant & $\begin{array}{l}\text { Oral ulcers; sore mouth; burns; colic; cuts; headache; and } \\
\text { uterine problems; dysentery; leprosy; malaria; insecticidal; } \\
\text { wormicidal; skin sores; diarrhoea; rheumatic swelling; } \\
\text { arthritis; skin disease; phlegm }\end{array}$ & General & $\begin{array}{l}\text { Kshirsagar and Singh 2001, } \\
\text { Reddy and Reddy } 2008\end{array}$ \\
\hline Anaphalis beddomei Hook. f. & Herb & Whole plant & Fever; cough; diarrhea. & General & Kanthasamy Kalaichelvi 2016 \\
\hline Artemisia absinthium $\mathrm{L}$. & Forb/herb & Whole plant & Tuberculosis; cough & General & Sharma et al. 2013 \\
\hline Artemisia annua L. & Forb/herb & Leaves & Cold; cough & General & Kayani et al. 2014 \\
\hline Artemisia brevifolia & Shrub & Whole plant & Cough; respiratory stimulant & General & Kayani et al. 2014 \\
\hline Artemisia lactiflora Wall. ex DC. & Shrub & Leaves & Tonic; post-labor tonic & $\begin{array}{l}\text { General; } \\
\text { women }\end{array}$ & Srithi et al. 2009 \\
\hline $\begin{array}{l}\text { Artemisia macrocephala Jacquem. ex } \\
\text { Besser }\end{array}$ & Herb & Seed & Cold; cough; flu; asthma & General & Kayani et al. 2014 \\
\hline Artemisia nilagirica (C.B. Clarke) Pamp. & Shrub & Whole plant & $\begin{array}{l}\text { Removing worms from wounds; joint and muscle to relieve } \\
\text { pain over boils; to prevent microbial infection; epilepsy; } \\
\text { diarrhoea; fever; mosquito repellent; Wounds; cuts; } \\
\text { scabies; and inflammations }\end{array}$ & $\begin{array}{l}\text { General; } \\
\text { animals }\end{array}$ & $\begin{array}{l}\text { Namsa et al. 2009, Sharma et } \\
\text { al. } 2013\end{array}$ \\
\hline Artemisia pallens Wall. ex DC. & Herb & Leaves & Antibacterial & General & Valsaraj et al. 1997 \\
\hline Artemisia scoparia Waldst. and Kitam. & Herb & Whole plant & Cough asthma; profuse mucous discharges & General & Kayani et al. 2014 \\
\hline Artemisia sphaerocephala Krasch. & Shrub & Gum, seed & Antidiabetic & General & Pandeya et al. 2013 \\
\hline Artemisia vulgaris $\mathrm{L}$. & Herb & Whole plant & $\begin{array}{l}\text { Appetizer and epilepsy; conjuctivitis; antibacterial; } \\
\text { Emmenagogue; Anthelmintic; respiratory disorders; fever }\end{array}$ & General & $\begin{array}{l}\text { Chopda and Mahajan 2009, } \\
\text { Valsaraj et al. } 1997 \\
\end{array}$ \\
\hline Aster tataricus L.f. & Herb & - & Anti-tumour & General & Zhang et al. 2013 \\
\hline Atractylodes macrocephala Koidz. & Herb & - & Anti-tumour & General & Zhang et al. 2013 \\
\hline $\begin{array}{l}\text { Ayapana triplinervis (Vahl) R.M. King and } \\
\text { H. Rob. }\end{array}$ & Herb & Leaves & Cuts & General & Udayan et al. 2005 \\
\hline Baccharoides anthelmintica (L.) Moench & Stout herb & $\begin{array}{l}\text { Leaves, } \\
\text { seeds, fruit, } \\
\text { root }\end{array}$ & $\begin{array}{l}\text { Anthelmintic; diabetes; fever; skin disease; body swelling } \\
\text { after delivery; snakebite }\end{array}$ & $\begin{array}{l}\text { General; } \\
\text { women }\end{array}$ & Harsha et al. 2003 \\
\hline Bidens biternata (Lour.) Merr. and Sherff & Herb & Whole plant & Cuts and wounds; antiseptic & General & $\begin{array}{l}\text { Jain et al. 2009, Kumar et al. } \\
2007\end{array}$ \\
\hline Bidens pilosa $\mathrm{L}$. & $\begin{array}{l}\text { Prostrate } \\
\text { herb }\end{array}$ & Leaves & $\begin{array}{l}\text { Fever; cough; wounds; skin diseases; stomachache; } \\
\text { vegetable; earache; Oedema; Snakebite; Subjugation }\end{array}$ & General & $\begin{array}{l}\text { Ayyanar and Ignacimuthu } 2005 \\
\text { Dansi et al. 2008, Namsa et al. } \\
2009\end{array}$ \\
\hline Blainvillea acmella (L.) Philipson & Herb & $\begin{array}{l}\text { Leaves, } \\
\text { seed }\end{array}$ & Skin cuts; Post-natal complaints; alcohol deaddiction & General & Jain et al. 2009 \\
\hline Blepharispermum petiolare DC. & Shrub & $\begin{array}{l}\text { Leaves, } \\
\text { bark }\end{array}$ & General & General & Dey and De 2012 \\
\hline Blumea balsamifera (L.) DC. & Undershrub & Leaves, root & Stomach ache; obstetric diseases & General & Srithi et al. 2009 \\
\hline Blumea fistulosa (Roxb.) Kurz & Herb & - & Poisonous sting of wasp & General & Namsa et al. 2009 \\
\hline Blumea mollis (D. Don) Merr. & Herb & Leaves & Cough & General & Sreeramulu et al. 2013 \\
\hline Caesulia axillaris Roxb. & Herb & Whole plant & Water sores & General & Dey et al. 2012 \\
\hline Calendula officinalis $\mathrm{L}$. & Herb & Flower & Snakebite & General & Samy et al. 2008 \\
\hline Calyptocarpus vialis Less. & Herb & Leaves, fruit & Fever; diarrhoea; laxatives & General & Kanthasamy Kalaichelvi 2016 \\
\hline
\end{tabular}

Published 11 February 2019

http://dx.doi.org/10.32859/era.18.4.1-112 


\begin{tabular}{|c|c|c|c|c|c|}
\hline Carthamus tinctorius $\mathrm{L}$. & Herb & $\begin{array}{l}\text { Flower, } \\
\text { seed }\end{array}$ & Cough; throat problems & General & Kayani et al. 2014 \\
\hline $\begin{array}{l}\text { Chromolaena odorata (L.) R.M. King and } \\
\text { H. Rob. }\end{array}$ & Shrub & $\begin{array}{l}\text { Leaves, } \\
\text { root, seed }\end{array}$ & $\begin{array}{l}\text { Malaria; wounds; vegetable; anti-bleeding; obstetric } \\
\text { diseases; gastric ulcers; wounds; skin infections; fever; } \\
\text { cough; skin injuries; breathing problems }\end{array}$ & General & $\begin{array}{l}\text { Ayyanar and Ignacimuthu } 2005 \text {, } \\
\text { Das et al. 2013, Namsa et al. } \\
2009\end{array}$ \\
\hline Chrysanthemum indicum $\mathrm{L}$. & Herb & Leaves & Earache; boils & General & Bhat et al. 2014 \\
\hline Cichorium endivia L. & Herb & Leaves & When throat hoarse & General & Parada et al. 2009 \\
\hline Cichorium intybus L. & Herb & Whole plant & Asthma & General & Kataria and Kaur 2013 \\
\hline $\begin{array}{l}\text { Conyza sumatrensis (S.F. Blake) Pruski } \\
\text { and G. Sancho }\end{array}$ & Herb & Leaves & Snake bite & General & Owuor et al. 2005 \\
\hline Conyza welwitschii (S. Moore) Wild & Herb & Whole plant & Toxemia & General & Chifundera 2001 \\
\hline $\begin{array}{l}\text { Crassocephalum crepidioides (Benth.) S. } \\
\text { Moore }\end{array}$ & Herb & $\begin{array}{l}\text { Leaves, } \\
\text { twigs }\end{array}$ & Vegetables and stomach disorder & General & Ruba and Mohan 2016 \\
\hline $\begin{array}{l}\text { Crassocephalum montuosum (S. Moore) } \\
\text { Milne-Redh. }\end{array}$ & Shrub & Whole plant & Diarrhea and placenta retention & $\begin{array}{l}\text { General; } \\
\text { women }\end{array}$ & Chifundera 2001 \\
\hline $\begin{array}{l}\text { Crassocephalum rubens (Juss. ex Jacq.) } \\
\text { S. Moore }\end{array}$ & Herb & Leaves & Vegetable & General & Dansi et al. 2008 \\
\hline Cyanthillium albicans (DC.) H. Rob. & Shrub & Leaves & Burns & General & Rajendran et al. 2000 \\
\hline Cyanthillium cinereum (L.) H. Rob. & Herb & Whole plant & $\begin{array}{l}\text { Rheumatism; malaria; intestinal worms; jaundice; abortion; } \\
\text { anti-inflammatory; antidiabetic; anticancer; antiviral; } \\
\text { antimicrobial; fever; cold; sore throat; headache; eczema; } \\
\text { bleeding; swelling; dysentery; dyspepsia; tranquilliser; } \\
\text { leucoderma; skin diseases; breast tumor; diarrhoea; } \\
\text { gastrointestinal disorders; cough; antibacterial }\end{array}$ & General & $\begin{array}{l}\text { Ayyanar and Ignacimuthu } 2005 \text {, } \\
\text { Iwalewa et al. 2003, Rao et al. } \\
\text { 2006, Valsaraj et al. } 1997\end{array}$ \\
\hline Dahlia coccinea Cav. & Herb & Tuber & Wound & General & Bhuvaneswari et al. 2015 \\
\hline Echinops ritrodes Bunge & Shrub & Root, leaves & Typhoid; rheumatoid arthritis; for easy delivery; cough & Women & Chopda and Mahajan 2009 \\
\hline Eclipta prostrata (L.) L. & Herb & Whole plant & $\begin{array}{l}\text { Malaria; baldness; ulcers; antiseptic; wounds; liver tonic; } \\
\text { jaundice; urinary disorders; skin diseases; menstural } \\
\text { disorder; hypertension; beri beri; snake bite; antibacterial; } \\
\text { intestinal worms; leucoderma; dandruff; diabetes; } \\
\text { diarrhoea; cough }\end{array}$ & $\begin{array}{l}\text { General; } \\
\text { cattle }\end{array}$ & $\begin{array}{l}\text { Chopda and Mahajan 2009, Dey } \\
\text { and De } 2012\end{array}$ \\
\hline Elephantopus scaber $\mathrm{L}$. & Herb & Whole plant & $\begin{array}{l}\text { Rheumatism; antibacterial; stomach ache; pyorhea; } \\
\text { asthma; headache; laxative; irregular mensturation; } \\
\text { anticancer; cuts; bruises; wounds; leprosy; bronchitis; } \\
\text { antibiotic; induce appetite; gastrointestinal problems; } \\
\text { eyesight improvement; to attain temporary male sterility }\end{array}$ & $\begin{array}{l}\text { General; } \\
\text { men }\end{array}$ & $\begin{array}{l}\text { Dubey et al. 2012, Reddy at al., } \\
\text { 2008, Xavier et al. } 2014\end{array}$ \\
\hline Emilia praetermissa Milne-Redh. & Herb & Leaves & Vegetable & General & Dansi et al. 2008 \\
\hline Emilia sonchifolia (L.) DC. ex DC. & Herb & Whole plant & $\begin{array}{l}\text { Vata; diarrhea; ophthalmia; cuts; wounds; intermittent } \\
\text { fever; asthma; antibacterial; eye infection; antisnake } \\
\text { venom }\end{array}$ & General & Nizar et al. 2015 \\
\hline Enydra fluctuans DC. & Herb & Whole plant & $\begin{array}{l}\text { Ascites; dropsy; inflammation; leucoderma; bronchitis; } \\
\text { tropidity of the liver; lack of appetite; and weakness due } \\
\text { typhoid fever; biliousness; skin disorders; nerve diseases; } \\
\text { anasarca; snakebite; hypertension }\end{array}$ & General & $\begin{array}{l}\text { Dey and De 2012, Eneh et al. } \\
2013\end{array}$ \\
\hline Erigeron trilobus (Decne.) Boiss. & Herb & Whole plant & Antibacterial activity & General & Ranjitha et al. 2016 \\
\hline
\end{tabular}

Published 11 February 2019

http://dx.doi.org/10.32859/era.18.4.1-112 
Ethnobotany Research and Applications

\begin{tabular}{|c|c|c|c|c|c|}
\hline Glossocardia bosvallia (L.f.) DC. & Herb & Whole plant & Typhoid & General & Porte 2014 \\
\hline Guizotia abyssinica (L.f.) Cass. & Herb & Root & Cattle neck inflammation & Cattle & Kshirsagar and Singh 2001 \\
\hline Gundelia tournefortii L. & Herb & - & - & - & Yazdanshenas et al. 2016 \\
\hline $\begin{array}{l}\text { Gymnanthemum amygdalinum (Delile) } \\
\text { Sch.Bip. ex Walp. }\end{array}$ & Tree & Leaves & Vegetable & General & Dansi et al. 2008 \\
\hline $\begin{array}{l}\text { Gymnanthemum coloratum (Willd.) H. } \\
\text { Rob. and B. Kahn }\end{array}$ & Tree & Leaves & Vegetable & General & Dansi et al. 2008 \\
\hline Gynura bicolor (Roxb. ex Willd.) DC. & Herb & Leaves & Tonic; post-labor tonic & General & Srithi et al. 2009 \\
\hline Helianthus annuus L. & Herb & Seed, oil & Snakebite & General & Patel 2014 \\
\hline Inula cappa (Buch. -Ham. ex D. Don) DC. & Shrub & Root & Epilepsy & General & Sharma et al. 2013 \\
\hline Inula obtusifolia A.Kerner & Herb & Whole plant & Tuberculosis; chest problems; cough & General & Kayani et al. 2014 \\
\hline Kleinia grandiflora (wallich ex DC.) N. Rani & Subshrub & Leaves, root & Gastric complaints & General & Ayyanar and Ignacimuthu 2005 \\
\hline Lactuca serriola L. & Herb & Whole plant & $\begin{array}{l}\text { Expectorant; cough; phthisis; bronchitis; jaundice; calcium } \\
\text { and iron source }\end{array}$ & $\begin{array}{l}\text { Pregnant } \\
\text { women }\end{array}$ & Rajasab and Isaq 2004 \\
\hline Lagascea mollis Cav. & Herb & Leaves & Swellings & General & Kosalge and Fursule 2009 \\
\hline Launaea intybacea (Jacq.) Beauverd & Herb & Leaves & Bronchitis & General & Savithramma et al. 2014 \\
\hline $\begin{array}{l}\text { Launaea procumbens (Roxb.) Ramayya } \\
\text { and Rajagopal }\end{array}$ & Herb & Leaves & To increase lactation & Women & Sayed et al. 2007 \\
\hline $\begin{array}{l}\text { Launaea sarmentosa (Willd.) Sch.Bip. ex } \\
\text { Kuntze }\end{array}$ & Trailing herb & - & Antidiarrhoeal & General & Tetali et al. 2009 \\
\hline $\begin{array}{l}\text { Launaea taraxacifolia (Willd.) Amin ex C. } \\
\text { Jeffrey }\end{array}$ & Herb & Leaves & Vegetable & General & Dansi et al. 2008 \\
\hline Matricaria chamomilla L. & Herb & - & $\begin{array}{l}\text { To clean sick eyes; to digest; for bellyache; for stomach } \\
\text { ache; for sore eyes; for sickness; to stop vomiting; for } \\
\text { wound disinfection }\end{array}$ & General & Parada et al. 2009 \\
\hline $\begin{array}{l}\text { Melanthera scandens (Schumach. and } \\
\text { Thonn.) Roberty }\end{array}$ & Herb & Leaves & Vegetable & General & Dansi et al. 2008 \\
\hline Mikania chenopodifolia Willd. & Herb & Leaves & Vegetable & General & Dansi et al. 2008 \\
\hline Parthenium hysterophorus L. & Herb & Whole plant & $\begin{array}{l}\text { Cuts; burns; dysentery; rheumatism; kidney stones; } \\
\text { febrifuge; neuralgia }\end{array}$ & General & $\begin{array}{l}\text { Rajendran et al. 2002, } \\
\text { Pradheeps and Poyyamoli } 2013\end{array}$ \\
\hline Pentanema indicum (L.) Ling & Herb & Whole plant & Skin disease & General & Ram et al. 2004 \\
\hline Pluchea indica (L.) Less. & Erect shrub & Seed, flower & Snakebite & General & Samy et al. 2008 \\
\hline Pluchea lanceolata (DC.) C.B. Clarke & Undershrub & Leaves & - & & Chopda and Mahajan 2009 \\
\hline Pulicaria wightiana (DC.) C.B. Clarke & Herb & Leaves & Fissures & General & Rao et al. 2006 \\
\hline Saussurea costus (Falc.) Lipsch. & Shrub & Root & Cough; asthma; throat problems; influenza & General & Kayani et al. 2014 \\
\hline Saussurea obvallata (DC.) Edgew. & Herb & $\begin{array}{l}\text { Flower, bud, } \\
\text { leaves }\end{array}$ & Cuts; wound; bruises; boils; cattle wounds & $\begin{array}{l}\text { General; } \\
\text { cattle }\end{array}$ & Kumar et al. 2007 \\
\hline Sclerocarpus africanus Jacq. ex Murray & Herb & Leaves & Vegetable & General & Dansi et al. 2008 \\
\hline Senecio chrysanthemoides DC. & Herb & $\begin{array}{l}\text { Root, flower, } \\
\text { leaves }\end{array}$ & Asthma; respiratory problems & General & Kayani et al. 2014 \\
\hline Senecio tenuifolius Burm. $\mathrm{f}$. & Herb & Leaves & Cuts; wounds & General & Rao et al. 2006 \\
\hline Sigesbeckia orientalis L. & $\begin{array}{l}\text { Erect } \\
\text { subshrub }\end{array}$ & Whole plant & $\begin{array}{l}\text { Wounds; parasitic skin problems; skin diseases; vitamin } \\
\text { difeciency; indigestion }\end{array}$ & General & $\begin{array}{l}\text { Deepak and Gopal 2014, Jain } \\
2004\end{array}$ \\
\hline Sonchus arvensis L. & Herb & Whole plant & Cough; bronchitis; asthma & General & Kayani et al. 2014 \\
\hline
\end{tabular}

Published 11 February 2019

http://dx.doi.org/10.32859/era.18.4.1-112 


\begin{tabular}{|c|c|c|c|c|c|}
\hline Sonchus asper (L.) Hill & Herb & Whole plant & Cough; bronchitis; asthma & General & Kayani et al. 2014 \\
\hline Sonchus oleraceus (L.) L. & Herb & Whole plant & Liver diseases; wound & General & Ramya 2008 \\
\hline Sphaeranthus indicus L. & Herb & Whole plant & $\begin{array}{l}\text { Snakebite; dysentry; skin diseases; asthma; impotency; } \\
\text { malaria; scabies }\end{array}$ & General & $\begin{array}{l}\text { Ram et al. 2004, Sharma et al. } \\
2013\end{array}$ \\
\hline Sphaeranthus senegalensis DC. & Herb & Fruit, root & Diarrhoea & General & Gairola et al. 2013 \\
\hline Sphagneticola calendulacea (L.) Pruski & Herb & $\begin{array}{l}\text { Leaves, } \\
\text { stem, root }\end{array}$ & $\begin{array}{l}\text { Hair blackening; jaundice; cough; cephalalgia; skin } \\
\text { diseases; alopecia }\end{array}$ & General & Ignacimuthu et al. 2008 \\
\hline Spilanthes acmella (L.) L. & Herb & $\begin{array}{l}\text { Root, flower, } \\
\text { leaves }\end{array}$ & Skin diseases; typhoid & General & Bhaskar and Samant 2012 \\
\hline Spilanthes oleracea L. & Herb & Leaves, twig & $\begin{array}{l}\text { Stop bleeding; skin infections and gastritis; fish poison; } \\
\text { malaria }\end{array}$ & General & Ruba and Mohan 2016 \\
\hline Synedrella nodiflora (L.) Gaertn. & Herb & Leaves & Swellings; vegetable & General & Rao et al. 2006 \\
\hline Tagetes erecta $\mathrm{L}$ & Herb & $\begin{array}{l}\text { Leaves, } \\
\text { flower }\end{array}$ & Cuts; boils; bruises; gastrointestinal disorders & General & Kumar et al. 2007 \\
\hline $\begin{array}{l}\text { Tanacetum cinerariifolium (Trevir.) } \\
\text { Sch.Bip. }\end{array}$ & Herb & Whole plant & Antidote to poison & General & Dey and De 2012 \\
\hline Taraxacum campylodes G.E. Haglund & Herb & Whole plant & Malaria; altitude sickness; snakebite & General & Dey and De 2012 \\
\hline Taraxacum tibetanum Hand.-Mazz. & Herb & $\begin{array}{l}\text { Leaves, } \\
\text { stem, flower }\end{array}$ & Vertigo; jaundice; gastritis; fever & General & Acharya and Kaphle 2015 \\
\hline Tridax procumbens (L.) L. & Herb & Leaves & $\begin{array}{l}\text { Antibacterial; antidiarrhoeal; boils; blisters; cuts; diarrhoea; } \\
\text { wound; cuts; bleeding; gastrointestinal disorders; } \\
\text { insecticidal; wormicidal; ulcers; anal fitsula; rheumatism; } \\
\text { swelling; skin diseases }\end{array}$ & General & $\begin{array}{l}\text { Rao et al. 2006, Umapriya et al. } \\
2011\end{array}$ \\
\hline Tussilago farfara L. & Herb & Whole plant & $\begin{array}{l}\text { Chronic bronchitis; asthma; chest complains; respiratory } \\
\text { infections; antiseptic; antiphlogistic }\end{array}$ & General & $\begin{array}{l}\text { Kayani et al. 2014, Zhang et al. } \\
2013\end{array}$ \\
\hline Vernonia amygdalina Delile & $\begin{array}{l}\text { Shrub to } \\
\text { small tree }\end{array}$ & Leaves & Malarial treatment & General & $\begin{array}{l}\text { Chenniappan and Kadarkarai } \\
2010 \\
\end{array}$ \\
\hline Vernonia arborea Buch. -Ham. & Tree & Leaves & - & General & Chopda and Mahajan 2009 \\
\hline $\begin{array}{l}\text { Vernonia conyzoides Hutchinson and } \\
\text { Dalziel }\end{array}$ & Subshrub & Leaves & To arrest bleeding & General & Manikandan 2005 \\
\hline Vernonia teres Wall. & Herb & Whole plant & - & General & Chopda and Mahajan 2009 \\
\hline Wedelia calendulacea Rich. & Herb & Leaves & Cold; cough; snakebite & General & Savithramma et al. 2014 \\
\hline Wedelia urticifolia (Blume) DC. & Herb & Whole plant & Jaundice & General & Kalaiselvan and Gopalan 2014 \\
\hline Xanthium pungens Wallr. & Subshrub & Leaves & Galactogogue & General & Sreeramulu et al. 2013 \\
\hline Xanthium strumarium $\mathrm{L}$. & Subshrub & $\begin{array}{l}\text { Stem, root, } \\
\text { leaves }\end{array}$ & Boils; heals injured muscle tissues; dogbite; weeping & $\begin{array}{l}\text { General; } \\
\text { children }\end{array}$ & Pradheeps and Poyyamoli 2013 \\
\hline \multicolumn{6}{|l|}{ Aytoniaceae } \\
\hline Reboulia hemisphaerica (L.) Raddi & Liverwort & - & $\begin{array}{l}\text { Used for blotches; hemostasis; external wounds; and } \\
\text { bruises }\end{array}$ & General & Chandra et al. 2016 \\
\hline \multicolumn{6}{|l|}{ Balanophoraceae } \\
\hline $\begin{array}{l}\text { Balanophora fungosa J.R. Forst. and G. } \\
\text { Forst. }\end{array}$ & Herb & Whole plant & Cures piles and internal haemorraghes & General & Pushpangadan and Atal 1984 \\
\hline \multicolumn{6}{|l|}{ Balsaminaceae } \\
\hline Impatiens balsamina L. & Herb & Flower, twig & Wounds; burns; scalds; boils; jaundice & General & Wagh and Jain 2015 \\
\hline
\end{tabular}

Published 11 February 2019

http://dx.doi.org/10.32859/era.18.4.1-112 
Ethnobotany Research and Applications

\begin{tabular}{|c|c|c|c|c|c|}
\hline $\begin{array}{l}\text { Impatiens diversifolia B. Heyne ex Wight } \\
\text { and Arn. }\end{array}$ & Herb & Whole plant & Epilepsy & General & $\begin{array}{l}\text { Muralidhara Rao and Pullaiah } \\
2007\end{array}$ \\
\hline Impatiens henslowiana Arn. & Herb & $\begin{array}{l}\text { Flower, } \\
\text { leaves }\end{array}$ & Jaundice & General & Asha and Pushpangadan 2002 \\
\hline \multicolumn{6}{|l|}{ Basellaceae } \\
\hline Basella alba L. & Climber & Leaves & $\begin{array}{l}\text { To decrease the hip pain during child birth; body heat; } \\
\text { tonic; post-labor tonic; Constipation; particularly in children } \\
\text { and pregnant women and in urinary disorders; mouth } \\
\text { ulcers; vegetable; piles; eye infections; skin boils; oral } \\
\text { health care }\end{array}$ & $\begin{array}{l}\text { General; } \\
\text { pregnant } \\
\text { women }\end{array}$ & $\begin{array}{l}\text { Bhat et al. 2014, Dansi et al. } \\
2008, \text { Hebbar et al. } 2004 \text {, Srith } \\
\text { et al. } 2009\end{array}$ \\
\hline \multicolumn{6}{|l|}{ Begoniaceae } \\
\hline Begonia crenata Dryand. & Herb & Whole plant & Acidity & \begin{tabular}{|c|} 
General \\
\end{tabular} & Kamble et al. 2008 \\
\hline Begonia malabarica Lam. & Herb & Whole plant & $\begin{array}{l}\text { For head ache and to cure wounds; fever; diabetes; } \\
\text { rheumatic pain; kidney stone; for stamina; nose infections; }\end{array}$ & General & $\begin{array}{l}\text { Ignacimuthu et al. 2008, } \\
\text { Pandikumar et al. } 2009\end{array}$ \\
\hline Begonia picta Sm. & Herb & Root & Sores in mouth & General & Rao et al. 2011 \\
\hline Begonia trichocarpa Dalzell & Herb & Leaves & Opacity & General & Jagtap et al. 2009 \\
\hline \multicolumn{6}{|l|}{ Berberidaceae } \\
\hline Berberis aristata DC. & Shrub & Whole plant & Typhoid; ophthalmic & General & Chopda and Mahajan 2009 \\
\hline Berberis tinctoria Lesch. & Shrub & Whole plant & $\begin{array}{l}\text { Hepatoprotective activity and antioxidant activity; diarrhea } \\
\text { and intestinal parasitic infections; snake bite; indigestion }\end{array}$ & General & $\begin{array}{l}\text { Banumathi and Vaseeharan } \\
\text { 2015, Deepak and Gopal } 2014\end{array}$ \\
\hline Mahonia fortunei (Lindl.) Fedde & Shrub & - & Anti-fungal & General & Zhang et al. 2013 \\
\hline $\begin{array}{l}\text { Sinopodophyllum hexandrum (Royle) T.S. } \\
\text { Ying }\end{array}$ & Herb & Rhizome & Liver diseases & General & Jain 2004 \\
\hline \multicolumn{6}{|l|}{ Bignoniaceae } \\
\hline Campsis radicans (L.) Seem. & Climber & Root & - & - & $\begin{array}{l}\text { Chopda and Mahajan 2009, } \\
\text { Srithi et al. } 2009\end{array}$ \\
\hline $\begin{array}{l}\text { Dolichandrone atrovirens (Roth) K. } \\
\text { Schum. }\end{array}$ & Tree & Bark & Stomach pain & General & Rao et al. 2012 \\
\hline $\begin{array}{l}\text { Dolichandrone falcata (Wall. ex DC.) } \\
\text { Seem. }\end{array}$ & Tree & $\begin{array}{l}\text { Bark, fruit, } \\
\text { leaves, stem }\end{array}$ & Corneal opacity; stomachache; leprosy; cough & $\begin{array}{l}\text { General; } \\
\text { children }\end{array}$ & $\begin{array}{l}\text { Dushing and Patil 2011, } \\
\text { Sreeramulu et al. } 2013\end{array}$ \\
\hline Dolichandrone spathacea (L.f.) Seem. & Tree & Bark & Bronchitis & General & Prasad et al. 2008 \\
\hline Mansoa alliacea (Lam.) A.H. Gentry & $\begin{array}{l}\text { Evergreen } \\
\text { climber }\end{array}$ & Root & Uterine disorders & Women & Prasad et al. 2014 \\
\hline Millingtonia hortensis L.f. & Tree & Root & $\begin{array}{l}\text { Indigestion; abdominal pain; fever; headache; dysentry; } \\
\text { diarrhoea; vomiting }\end{array}$ & General & Chander et al. 2015 \\
\hline Oroxylum indicum (L.) Kurz & Small tree & $\begin{array}{l}\text { Seed, stem, } \\
\text { leaves, } \\
\text { flower, root, } \\
\text { bark, fruit } \\
\end{array}$ & $\begin{array}{l}\text { Contraceptive; epilepsy; antibacterial; diarrhoea; } \\
\text { dysentery; Fits; Leucorrhoea; Menorrhagia; Tuberculosis; } \\
\text { chicken pox; snakebite; centipede bite; rheumatism; } \\
\text { gynaecological disorders; scabies; leprosy; pyorrhea }\end{array}$ & $\begin{array}{l}\text { General; } \\
\text { women }\end{array}$ & $\begin{array}{l}\text { Das et al. 2014, Dubey et al. } \\
\text { 2012, Lingaraju et al. } 2013\end{array}$ \\
\hline Pajanelia Iongifolia (Willd.) K. Schum. & Tree & Leaves & Boils & General & Prasad et al. 2008 \\
\hline $\begin{array}{l}\text { Radermachera xylocarpa (Roxb.) Roxb. } \\
\text { ex K. Schum. }\end{array}$ & Tree & Bark & Weakness; skin burns; rheumatoid arthritis & General & Kshirsagar and Singh 2001 \\
\hline Spathodea campanulata P. Beauv. & Tree & $\begin{array}{l}\text { Flower, } \\
\text { stem bark, } \\
\text { leaves }\end{array}$ & $\begin{array}{l}\text { Diuretic; anti-inflammatory; kidney diseases; urethra } \\
\text { inflammations and as an antidote against animal poisons; }\end{array}$ & General & Dhanabalan 2008 \\
\hline
\end{tabular}

Published 11 February 2019

http://dx.doi.org/10.32859/era.18.4.1-112 


\begin{tabular}{|c|c|c|c|c|c|}
\hline & & & $\begin{array}{l}\text { enemas; fungus skin diseases; herpes; stomachaches and } \\
\text { diarrhea }\end{array}$ & & \\
\hline Stereospermum chelonoides (L.f.) DC. & Tree & Leaves, root & Menstural disorders; wounds; increases fertility & $\begin{array}{l}\text { General; } \\
\text { women }\end{array}$ & $\begin{array}{l}\text { Jain et al. 2004, Udayan et al. } \\
2007\end{array}$ \\
\hline Stereospermum tetragonum DC. & Tree & Bark & - & General & Nizar et al. 2015 \\
\hline Tecoma stans (L.) Juss. ex Kunth & Small tree & Leaves & Anti-bacterial activity & General & Suganthi and Libina 2015 \\
\hline Tecomella undulata (Sm.) Seem. & Tree & $\begin{array}{l}\text { Bark, stem, } \\
\text { root }\end{array}$ & Abortifacient; syphilis; leucorrhoea; diabetes & $\begin{array}{l}\text { General; } \\
\text { pregnant } \\
\text { women }\end{array}$ & Jain et al. 2004 \\
\hline \multicolumn{6}{|l|}{ Bixaceae } \\
\hline Bixa orellana L. & $\begin{array}{l}\text { Shrub to } \\
\text { small tree }\end{array}$ & $\begin{array}{l}\text { Root, fruit, } \\
\text { seed, leaves }\end{array}$ & $\begin{array}{l}\text { Kidney stone symptoms; Insect repellant; Appetite } \\
\text { stimulant; dysentery; diarrhoea; sanke bite; fever }\end{array}$ & General & $\begin{array}{l}\text { Padal et al. 2014, Reddy et al. } \\
2008, \text { Rao et al. } 2006\end{array}$ \\
\hline $\begin{array}{l}\text { Cochlospermum planchonii Hook. f. ex } \\
\text { Planch. }\end{array}$ & Shrub & Leaves & Used as leafy vegetable & General & Dansi et al. 2008 \\
\hline Cochlospermum religiosum (L.) Alston & Tree & $\begin{array}{l}\text { Fruit, } \\
\text { leaves, root }\end{array}$ & Gonorrhea and dysentry; piles; chicken pox & General & $\begin{array}{l}\text { Rao et al. 2006, Savithramma et } \\
\text { al. 2014, Sreeramulu et al. } 2013\end{array}$ \\
\hline \multicolumn{6}{|l|}{ Blechnaceae } \\
\hline Blechnum orientale L. & Fern & $\begin{array}{l}\text { Rhizome, } \\
\text { aerial parts }\end{array}$ & Relief pain and bleeding; typhoid & General & Namsa et al. 2009 \\
\hline \multicolumn{6}{|l|}{ Boraginaceae } \\
\hline Coldenia procumbens $\mathrm{L}$. & Herb & Whole plant & Antirheumatic; rheumatic swellings; to improve resistance & $\begin{array}{l}\text { General; } \\
\text { children }\end{array}$ & Ignacimuthu et al. 2008 \\
\hline Cordia africana Lam. & Tree & Bark & Madness; mouth ulcer; antiinflammatory & General & Chifundera 2001 \\
\hline Cordia monoica Roxb. & Tree & Leaves & Chest pains & General & Pradheeps and Poyyamoli 2013 \\
\hline Cordia sinensis Lam. & Tree & $\begin{array}{l}\text { Leaves, } \\
\text { fruit, wood }\end{array}$ & Anti-inflammatory; Blood pressure; firewood & General & Kanthasamy Kalaichelvi 2016 \\
\hline Ehretia microphylla Lam. & Shrub & $\begin{array}{l}\text { Leaves, } \\
\text { fruit, root }\end{array}$ & Toothache and strength of teeth; menstural disorders & General & $\begin{array}{l}\text { Ayyanar and Ignacimuthu 2005, } \\
\text { Kanthasamy Kalaichelvi } 2016\end{array}$ \\
\hline $\begin{array}{l}\text { Cynoglossum wallichii var. glochidiatum } \\
\text { (Wall. ex Benth.) Kazmi }\end{array}$ & Herb & Root & Wounds & General & Dey et al. 2012 \\
\hline Cynoglossum zeylanicum (Vahl) Brand & Herb & Root & Snake-bite & General & Kshirsagar and Singh 2001 \\
\hline Ehretia acuminata R. Br. & Tree & Bark & Fever & General & Kanthasamy Kalaichelvi 2016 \\
\hline Ehretia cymosa Thonn. & Tree & Leaves & Vegetable & General & Dansi et al. 2008 \\
\hline Ehretia laevis Roxb. & Tree & Fruit, leaves & Edible & General & Bhagat et al. 2016 \\
\hline Ehretia microphylla Lam. & Tree & Fruit, root & Diarrhoea; snake bite & General & Samy et al. 2008 \\
\hline Heliotropium bracteatum R. Br. & Herb & Leaves & Stomach ulcers & General & Savithramma et al. 2014 \\
\hline Heliotropium indicum $\mathrm{L}$. & Herb & Whole plant & $\begin{array}{l}\text { Diabetic; dandruff; vegetable; skin disorders; malaria; } \\
\text { typhoid }\end{array}$ & General & $\begin{array}{l}\text { Dansi et al. 2008, Kumar et al. } \\
\text { 2007, Lingaraju et al. } 2013\end{array}$ \\
\hline Heliotropium marifolium J. Koenig ex Retz. & Herb & - & Snakebite & General & Dey and De 2012 \\
\hline Heliotropium ovalifolium Forssk. & Herb & Leaves & Insect stings & General & Raju 2008 \\
\hline Rotula aquatica Lour. & $\begin{array}{l}\text { Woody } \\
\text { shrub }\end{array}$ & Bark, root & Shivering; urinary problems; kidney stones; body prickles & General & Udayan et al. 2005 \\
\hline Trichodesma indicum (L.) Lehm. & Herb & Whole plant & $\begin{array}{l}\text { Snake bites; cough; diarrhoea; dysentery; influenza; } \\
\text { swelling of joints; wound; ear pain }\end{array}$ & General & Kumar et al. 2007 \\
\hline
\end{tabular}

Published 11 February 2019

http://dx.doi.org/10.32859/era.18.4.1-112 


\begin{tabular}{|c|c|c|c|c|c|}
\hline Trichodesma zeylanicum (Burm. f.) R. Br. & Herb & Root, leaves & $\begin{array}{l}\text { Skin diseases; dysentery; rheumatism; anthelmintic; } \\
\text { snakebite }\end{array}$ & General & Deepak and Gopal 2014 \\
\hline Heliotropium eichwaldii Steud. & Herb & Leaves & - & - & Chopda and Mahajan 2009 \\
\hline \multicolumn{6}{|l|}{ Brassicaceae } \\
\hline Brassica juncea (L.) Czern. & Herb & $\begin{array}{l}\text { Seed, } \\
\text { leaves, root }\end{array}$ & $\begin{array}{l}\text { Anti diabetic; fever and headache; dysentery; Gingival } \\
\text { wounds }\end{array}$ & General & $\begin{array}{l}\text { Bhat et al. 2012, Kadhirvel et al. } \\
2010\end{array}$ \\
\hline Brassica rapa L. & Herb & Whole plant & Wounds treatment & General & Dey and De 2012 \\
\hline Descurainia sophia (L.) Webb ex Prantl & Herb & $\begin{array}{l}\text { Flower, } \\
\text { leaves, seed }\end{array}$ & Chest complain; cough & General & Kayani et al. 2014 \\
\hline Lepidium sativum $\mathrm{L}$. & Small herb & Whole plant & Bronchitis; increases lactation & $\begin{array}{l}\text { General; } \\
\text { women }\end{array}$ & Nanjunda 2010 \\
\hline Lobularia maritima (L.) Desv. & Herb & $\begin{array}{l}\text { Flowered } \\
\text { aerial part }\end{array}$ & For stones in the kidney(renal lithotripter) & General & Parada et al. 2009 \\
\hline $\begin{array}{l}\text { Raphanus raphanistrum subsp. sativus } \\
\text { (L.) Domin }\end{array}$ & Herb & Seed & Amenorrhoea; abnormal menstruation & $\begin{array}{l}\text { General; } \\
\text { women }\end{array}$ & Patel 2010 \\
\hline Rorippa indica (L.) Hiern & Herb & Whole plant & Ear diseases & General & Puravankara and Gopal 2012 \\
\hline Sisymbrium erysimoides Desf. & Herb & Whole plant & Expectorant; bronchial disorders; sore throat & General & Kayani et al. 2014 \\
\hline \multicolumn{6}{|l|}{ Bryaceae } \\
\hline Rhodobryum giganteum (Schwägr.) Paris & Bryophyte & - & $\begin{array}{l}\text { Used to treat cardiovascular problem and nervous } \\
\text { prostration; to cure angina; anti-hypoxia; diuretic; } \\
\text { antipyretic; and antihypertensive }\end{array}$ & General & Chandra et al. 2016 \\
\hline Rhodobryum roseum (Hedw.) Limpr. & Bryophyte & - & $\begin{array}{l}\text { Used to treat nervous prostration and cardiovascular } \\
\text { diseases sedative }\end{array}$ & General & Chandra et al. 2016 \\
\hline \multicolumn{6}{|l|}{ Burseraceae } \\
\hline $\begin{array}{l}\text { Boswellia ovalifoliolata N.P. Balakr. and } \\
\text { A.N. Henry }\end{array}$ & Tree & $\begin{array}{l}\text { Gum, bark, } \\
\text { leaves, } \\
\text { stem, seed }\end{array}$ & $\begin{array}{l}\text { Ulcers; rheumatic pains; amoebic dysentery; hydrocoel in } \\
\text { testicle; mosquito repellent; ulcers; diarrhoea }\end{array}$ & General & $\begin{array}{l}\text { Muralidhara Rao and Pullaiah } \\
2007\end{array}$ \\
\hline Boswellia serrata Roxb. ex Colebr. & Tree & Whole plant & $\begin{array}{l}\text { Rheumatism; dog bite; scorpion sting; cough; diseases; } \\
\text { nervous and cutaneous disorders; impotency; antidote; } \\
\text { dysentery; arthritis; snake bite; menorrhegia }\end{array}$ & $\begin{array}{l}\text { Men; } \\
\text { General }\end{array}$ & $\begin{array}{l}\text { Chopda and Mahajan 2009, Jain } \\
\text { et al. 2004, Rao et al. } 2006\end{array}$ \\
\hline Canarium strictum Roxb. & Large tree & $\begin{array}{l}\text { Seed, resin, } \\
\text { bark }\end{array}$ & $\begin{array}{l}\text { On skin area exposed to poisonous hairs of caterpillar } \\
\text { larvae; heel cracks; mosquito repel; for resin }\end{array}$ & General & $\begin{array}{l}\text { Arjunan et al. 2016, Namsa et } \\
\text { al. } 2009\end{array}$ \\
\hline $\begin{array}{l}\text { Commiphora caudata (Wight and Arn.) } \\
\text { Engl. }\end{array}$ & Tree & $\begin{array}{l}\text { Bark, } \\
\text { leaves, } \\
\text { seed, gum }\end{array}$ & $\begin{array}{l}\text { Anti-viral;anti inflammatory; analgesic; scorpion sting; cuts; } \\
\text { boils }\end{array}$ & General & $\begin{array}{l}\text { Arjunan et al. 2016, Kanthasamy } \\
\text { Kalaichelvi } 2016\end{array}$ \\
\hline $\begin{array}{l}\text { Commiphora mukul (Hook. ex Stocks) } \\
\text { Engl. }\end{array}$ & Herb & Gum & Rheumatoid arthritis & General & Mallikarjuna 2015 \\
\hline Commiphora wightii (Arn.) Bhandari & Shrub & Gum & Cough; lung congestion & General & Meena and Yadav 2010 \\
\hline Garuga pinnata Roxb. & $\begin{array}{l}\text { Decidious } \\
\text { tree }\end{array}$ & $\begin{array}{l}\text { Bark, fruit, } \\
\text { leaves }\end{array}$ & $\begin{array}{l}\text { Wounds; asthma; diarrhea; conjunctivitis; and pulmonary } \\
\text { infections; dysentery; toothache; leucorrhea; burns; goiter }\end{array}$ & General & $\begin{array}{l}\text { Bhagat et al. 2016, Sreeramulu } \\
\text { et al. } 2013\end{array}$ \\
\hline \multicolumn{6}{|l|}{ Cactaceae } \\
\hline Cereus pterogonus Lem. & Shrub & Whole plant & Refrigerant; anti-periodic and antipyretic. & General & Kanthasamy Kalaichelvi 2016 \\
\hline Opuntia dillenii (Ker Gawl.) Haw. & Shrub & Whole plant & $\begin{array}{l}\text { Body sores; asthma; whooping cough; fever; constipation; } \\
\text { conjulcers; liver complaints; gastrointestinal disorders }\end{array}$ & $\begin{array}{l}\text { General; } \\
\text { women }\end{array}$ & Ayyanar and Ignacimuthu 2010 \\
\hline
\end{tabular}

Published 11 February 2019

http://dx.doi.org/10.32859/era.18.4.1-112 


\begin{tabular}{|c|c|c|c|c|c|}
\hline Opuntia elatior Mill. & Shrub & Whole plant & Increases lactation; edible & $\begin{array}{l}\text { General; } \\
\text { women }\end{array}$ & Kataria and Kaur 2013 \\
\hline Opuntia ficus-indica (L.) Mill. & Shrub & Fruit, leaves & Asthma; whooping cough; heat; inflammation; ulcers & General & Ram et al. 2016 \\
\hline Opuntia stricta (Haw.) Haw. & Shrub & $\begin{array}{l}\text { Stem, } \\
\text { leaves }\end{array}$ & Boils; blisters; snake bite & General & Rao et al. 2006 \\
\hline \multicolumn{6}{|l|}{ Calceolariaceae } \\
\hline Calceolaria mexicana Benth. & Herb & Whole plant & Antibacterial activity & General & Ranjitha et al. 2016 \\
\hline \multicolumn{6}{|l|}{ Calophyllaceae } \\
\hline Mesua ferrea L. & Large tree & $\begin{array}{l}\text { Flower, } \\
\text { leaves, } \\
\text { stamen }\end{array}$ & Postpartum health; snakebite & General & Dey and De 2012 \\
\hline Mammea longifolia Planch. and Triana & Tree & Bark & Antibacterial & General & Valsaraj et al. 1997 \\
\hline $\begin{array}{l}\text { Mammea suriga (Buch. -Ham. ex Roxb.) } \\
\text { Kosterm. }\end{array}$ & Tree & $\begin{array}{l}\text { Bark, flower } \\
\text { bud }\end{array}$ & Eczema & General & $\begin{array}{l}\text { Muralidhara Rao and Pullaiah } \\
2007\end{array}$ \\
\hline \multicolumn{6}{|l|}{ Campanulaceae } \\
\hline Codonopsis pilosula (Franch.) Nannf. & $\begin{array}{l}\text { Perennial } \\
\text { climber }\end{array}$ & - & Anti-microbial; anti-oxidant & General & Zhang et al. 2013 \\
\hline Lobelia chinensis Lour. & Shrub & - & Anti-cancer & General & Zhang et al. 2013 \\
\hline Lobelia heyneana Schult. & Herb & Leaves & Skin diseases & General & Ignacimuthu et al. 2008 \\
\hline Lobelia nicotianifolia Roth ex Schult. & Herb & $\begin{array}{l}\text { Leaves, } \\
\text { latex, root }\end{array}$ & $\begin{array}{l}\text { Snake bite; skin infections; insecticide; tooth decay; skin } \\
\text { diseases; boils }\end{array}$ & General & Lingaraju et al. 2013 \\
\hline Platycodon grandiflorus (Jacq.) A.DC. & Herb & - & Anti-inflammatory & General & Zhang et al. 2013 \\
\hline \multicolumn{6}{|l|}{ Cannabaceae } \\
\hline Cannabis sativa L. & $\begin{array}{l}\text { Tall erect } \\
\text { herb }\end{array}$ & $\begin{array}{l}\text { Leaves, } \\
\text { seed, bark, } \\
\text { flower }\end{array}$ & $\begin{array}{l}\text { to treat malaria and malaria-like symptoms; blood in } \\
\text { excreta; insecticidal; wormicidal; psychoactive drug; } \\
\text { Throat infection; chest problems; Veterinary; } \\
\text { Gynaecological disorders }\end{array}$ & $\begin{array}{l}\text { General; } \\
\text { animals; } \\
\text { women }\end{array}$ & $\begin{array}{l}\text { Bhardwaj et al. 2011, Kayani et } \\
\text { al. 2014, Padal et al. } 2014\end{array}$ \\
\hline Celtis australis L. & Tree & Whole plant & To low blood pressure; cough & General & Kayani et al. 2014 \\
\hline Celtis philippensis Blanco & Small tree & Bark & Cuts; wound healing; digestion problems & General & Prasad et al. 2008 \\
\hline Celtis timorensis Span. & Tree & Wood & Firewood & General & Jayakumar et al. 2010 \\
\hline Trema orientalis (L.) Blume & $\begin{array}{l}\text { Small tree or } \\
\text { large shrub }\end{array}$ & Whole plant & Diabetes; respiratory diseases; fever; vomiting; cough & General & Puravankara and Gopal 2012 \\
\hline \multicolumn{6}{|l|}{ Cannaceae } \\
\hline Canna indica L. & Herb & $\begin{array}{l}\text { Stem, tuber, } \\
\text { rhizome, } \\
\text { root }\end{array}$ & $\begin{array}{l}\text { Diuretic and digestion; dyspepsia; vegetable; urinary } \\
\text { diorder; fever }\end{array}$ & General & $\begin{array}{l}\text { Kalaiselvan and Gopalan 2014, } \\
\text { Padal et al. } 2010\end{array}$ \\
\hline \multicolumn{6}{|l|}{ Capparaceae } \\
\hline Cadaba fruticosa (L.) Druce & Shrub & Bark, leaves & Skin boils; Blisters; Cuts; dysentery & General & Rao et al. 2006 \\
\hline Cadaba trifoliata Wight and Arn. & Shrub & $\begin{array}{l}\text { Leaves, } \\
\text { stem, root }\end{array}$ & Anti-rheumatic; anthelmintic; antibacterial; viral infection & General & Kanthasamy Kalaichelvi 2016 \\
\hline Capparis cartilaginea Decne. & Shrub & $\begin{array}{l}\text { Latex, } \\
\text { leaves }\end{array}$ & Asthma & General & Kayani et al. 2014 \\
\hline Capparis decidua (Forssk.) Edgew. & Shrub & $\begin{array}{l}\text { Stem, root, } \\
\text { seed, fruit, } \\
\text { bark }\end{array}$ & $\begin{array}{l}\text { Pyorrhea and rheumatism; tumor; asthma; cough; } \\
\text { muscular injuries; antidote; Cough; Asthma; bronchial } \\
\text { problems }\end{array}$ & General & $\begin{array}{l}\text { Dey and De 2012, Kayani et al. } \\
2014\end{array}$ \\
\hline
\end{tabular}

Published 11 February 2019

http://dx.doi.org/10.32859/era.18.4.1-112 
Ethnobotany Research and Applications

\begin{tabular}{|c|c|c|c|c|c|}
\hline Capparis divaricata Lam. & $\begin{array}{l}\text { Shrub to } \\
\text { small tree }\end{array}$ & Bark, leaves & Dysentery; Stomach Problems & General & $\begin{array}{l}\text { Manikandan and Lakshmanan } \\
2014\end{array}$ \\
\hline Capparis diversifolia Wight and Arn. & Shrub & Leaves & Fever; Cold & General & Kanthasamy Kalaichelvi 2016 \\
\hline $\begin{array}{l}\text { Capparis nilgiriensis Subba Rao, Kumari } \\
\text { and V. Chandras. }\end{array}$ & Shrub & Leaves, root & Fever, to cure wounds of cattle & $\begin{array}{l}\text { General; } \\
\text { veterinary }\end{array}$ & $\begin{array}{l}\text { Muralidhara Rao and Pullaiah } \\
2007\end{array}$ \\
\hline Capparis olacifolia Hook. f. and Thomson & $\begin{array}{l}\text { Shrub to } \\
\text { small tree }\end{array}$ & Root & To cure pain and pus formation in ear & General & $\begin{array}{l}\text { Muralidhara Rao and Pullaiah } \\
2007\end{array}$ \\
\hline Capparis roxburghii DC. & Climber & Leaves & $\begin{array}{l}\text { Skin diseases; dropsy and gout; mumps; swelling of eyes; } \\
\text { blood purifier; stomachic; tonic and appetizer; cough and } \\
\text { toxemia; tumours; inflammation and diseases of the } \\
\text { muscles; treatment of aphthae; headache; eczema; } \\
\text { dandruff and to reduce the body heat skin disease }\end{array}$ & General & $\begin{array}{l}\text { Muralidhara Rao and Pullaiah } \\
2007\end{array}$ \\
\hline Capparis sepiaria L. & $\begin{array}{l}\text { Straggling } \\
\text { shrub }\end{array}$ & Leaves & Oral health care & General & Hebbar et al. 2004 \\
\hline Capparis zeylanica L. & $\begin{array}{l}\text { Straggling } \\
\text { shrub }\end{array}$ & $\begin{array}{l}\text { Bark, fruit, } \\
\text { leaves }\end{array}$ & $\begin{array}{l}\text { Snakebite; boils and swellings.; diabetes; ulcers; paralysis; } \\
\text { gastrointestinal disorder; dysentery; diarrhoea }\end{array}$ & General & $\begin{array}{l}\text { Reddy et al. 2008, Rao et al. } \\
2006 \text {, Tetali et al. } 2009\end{array}$ \\
\hline Capparis olacifolia Hook. f. and Thomson & $\begin{array}{l}\text { Shrub to } \\
\text { small tree }\end{array}$ & Root & Pain and pus formation in ear & General & $\begin{array}{l}\text { Muralidhara Rao and Pullaiah } \\
2007\end{array}$ \\
\hline Crateva adansonii DC. & Tree & Bark, leaves & Wound; joint pain; breast milk secretion; vegetable & $\begin{array}{l}\text { General; } \\
\text { women }\end{array}$ & Kanthasamy Kalaichelvi 2016 \\
\hline Crateva religiosa G. Forst. & Tree & $\begin{array}{l}\text { Flower, twig, } \\
\text { bark }\end{array}$ & Urinary complaints; snake bite; tympany; abortion & $\begin{array}{l}\text { General; } \\
\text { women; } \\
\text { pregnant } \\
\text { women }\end{array}$ & Kanthasamy Kalaichelvi 2016 \\
\hline Maerua oblongifolia (Forssk.) A. Rich. & $\begin{array}{l}\text { Woody } \\
\text { climber }\end{array}$ & Tuber & Fertility & General & Sreeramulu et al. 2013 \\
\hline \multicolumn{6}{|l|}{ Caprifoliaceae } \\
\hline Lonicera japonica Thunb. & Shrub & Stem, flower & Respiratory tract; skin diseases; tumors & General & Kanthasamy Kalaichelvi 2016 \\
\hline Nardostachys jatamansi (D. Don) DC. & Herb & $\begin{array}{l}\text { Rhizome, } \\
\text { root }\end{array}$ & Epilepsy & General & Sharma et al. 2013 \\
\hline Valeriana hardwickii Wall. & Herb & Whole plant & - & General & Sharma et al. 2013 \\
\hline Valeriana himalayana Grubov & Herb & Root & Cough; asthma & General & Kayani et al. 2014 \\
\hline Valeriana jatamansi Jones & Herb & Root & - & General & Sharma et al. 2013 \\
\hline \multicolumn{6}{|l|}{ Cardiopteridaceae } \\
\hline Gonocaryum lobbianum (Miers) Kurz & Shrub & Leaves & Treating obstetric diseases or body pain & General & Srithi et al. 2009 \\
\hline \multicolumn{6}{|l|}{ Caricaceae } \\
\hline Carica papaya L. & Tree & $\begin{array}{l}\text { Latex, fruit, } \\
\text { leaves, seed }\end{array}$ & $\begin{array}{l}\text { Used to gum; skin diseases; scorpion sting and snake bite; } \\
\text { edible; antibacterial; to prevent spontaneous abortion; } \\
\text { vitamin C source; abortifacient; inflammatory pain; malaria; } \\
\text { pimples; contraceptive; skin irritations; gynaecological } \\
\text { disorders }\end{array}$ & General & $\begin{array}{l}\text { Bhat et al. 2014, Dey et al. } \\
\text { 2012, Hebbar et al. 2004, } \\
\text { Valsaraj et al. } 1997\end{array}$ \\
\hline \multicolumn{6}{|l|}{ Caryophyllaceae } \\
\hline Drymaria cordata (L.) Willd. ex Schult. & Herb & Whole plant & Cuts; burns and wounds & General & $\begin{array}{l}\text { Kumar et al. 2007, } \\
\text { Ramachandran et al. } 2009\end{array}$ \\
\hline Polycarpaea aurea Wight and Arn. & Herb & Whole plant & Diabetes & General & Savithramma et al. 2014 \\
\hline
\end{tabular}

Published 11 February 2019

http://dx.doi.org/10.32859/era.18.4.1-112 
Ethnobotany Research and Applications

\begin{tabular}{|c|c|c|c|c|c|}
\hline Polycarpaea corymbosa (L.) Lam. & Herb & Whole plant & Liver diseases; jaundice; boils; inflammatory swellings & General & Jain 2004 \\
\hline Pseudostellaria heterophylla (Miq.) Pax & Herb & - & Anti-fungal & General & Zhang et al. 2013 \\
\hline \multicolumn{6}{|l|}{ Casuarinaceae } \\
\hline Casuarina equisetifolia L. & Tree & Bark & Skin diseases & General & Harsha et al. 2008 \\
\hline \multicolumn{6}{|l|}{ Celastraceae } \\
\hline Cassine glauca (Rottb.) Kuntze & Tree & $\begin{array}{l}\text { Bark, fruit, } \\
\text { root }\end{array}$ & Snake bite; fire wood; leucorrhoea & General & Sharma et al. 2013 \\
\hline Celastrus paniculatus Willd. & Climber & $\begin{array}{l}\text { Flowers, } \\
\text { leaves, root, } \\
\text { seed, bark, } \\
\text { seed oil }\end{array}$ & $\begin{array}{l}\text { Used as nerve stimulant and brain tonic; wound; body } \\
\text { pain; insect bite; leucorrhea; digestive stimulant; edible; } \\
\text { aphrodisiac; burning sensation; itching due to impure } \\
\text { blood }\end{array}$ & $\begin{array}{l}\text { General; } \\
\text { women }\end{array}$ & $\begin{array}{l}\text { Bhat et al. 2014, Reddy et al. } \\
2008, \text { Rao et al. 2006, Sharma } \\
\text { et al. } 2013\end{array}$ \\
\hline Euonymus cochinchinensis Pierre & Shrub & Aerial parts & Treating dysuria or body pain & General & Srithi et al. 2009 \\
\hline Gymnosporia emarginata (Willd.) Thwaites & Shrub & Bark, leaves & Snake bite; hepatitis & General & Padma et al. 2016 \\
\hline Gymnosporia montana (Roth) Benth. & Shrub & Leaves & Stomach ulcers & General & Savithramma et al. 2014 \\
\hline $\begin{array}{l}\text { Gymnosporia royleana Wall. ex M.A. } \\
\text { Lawson }\end{array}$ & Shrub & Root & - & - & Dey and De 2012 \\
\hline Gymnosporia senegalensis (Lam.) Loes. & Shrub & Aerial parts & Contraceptive; vegetable & $\begin{array}{l}\text { General; } \\
\text { women }\end{array}$ & Bhogaonkar and Kadam 2006 \\
\hline Parnassia nubicola Wall. ex Royle & Herb & Root & Wound & Veterinary & Dey and De 2012 \\
\hline Pleurostylia opposita (Wall.) Alston & Tree & - & Fodder plant; fire wood & General & Prabakaran et al. 2013 \\
\hline $\begin{array}{l}\text { Maytenus heyneana (Roth) D.C.S. Raju } \\
\text { and Babu }\end{array}$ & Shrub & Root, leaves & Dysentery & General & Rao et al. 2006 \\
\hline Salacia reticulata Wight & $\begin{array}{l}\text { Scandent } \\
\text { shrub }\end{array}$ & Whole plant & Girdle pain & General & Nizar et al. 2015 \\
\hline \multicolumn{6}{|l|}{ Ceratophyllaceae } \\
\hline Ceratophyllum demersum L. & Herb & Whole plant & $\begin{array}{l}\text { Used as purgative; astringent; constipating and antipyratic; } \\
\text { scorpion stings }\end{array}$ & General & $\begin{array}{l}\text { Al-Asmari et al. 2016, Chandra } \\
\text { et al. } 2016\end{array}$ \\
\hline \multicolumn{6}{|c|}{ 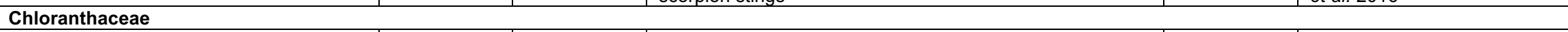 } \\
\hline Chloranthus elatior Link & Shrub & Stem & Rapid healing & General & Namsa et al. 2009 \\
\hline Sarcandra glabra (Thunb.) Nakai & Shrub & - & Anti-microbial & General & Zhang et al. 2013 \\
\hline \multicolumn{6}{|l|}{ Chlorellaceae } \\
\hline Chlorella vulgaris Beyerinck & Algae & - & On burnt skin due to fire and hot water & General & Namsa et al. 2009 \\
\hline \multicolumn{6}{|l|}{ Cistaceae } \\
\hline Cistus albidus $\mathrm{L}$. & Shrub & $\begin{array}{l}\text { Leaves, } \\
\text { flowered } \\
\text { aerial part }\end{array}$ & For cough & General & Parada et al. 2009 \\
\hline \multicolumn{6}{|l|}{ Cleomaceae } \\
\hline Cleome gynandra L. & Herb & $\begin{array}{l}\text { Leaves, } \\
\text { bark, seed, } \\
\text { root }\end{array}$ & $\begin{array}{l}\text { Ear ache; vegetable; insecticidal; wormicidal; wound; oral } \\
\text { health care; snakebite; diuretic; cough; malarial fevers; } \\
\text { cold }\end{array}$ & General & $\begin{array}{l}\text { Bhardwaj et al. 2011, Dansi et } \\
\text { al. } 2008\end{array}$ \\
\hline Cleome monophylla L. & Herb & Leaves & Headache & General & Ignacimuthu et al. 2008 \\
\hline Cleome viscosa L. & Herb & Whole plant & $\begin{array}{l}\text { Ear diseases; for flatulence; indigestion; stomach pain and } \\
\text { body pain; headache; boils; wounds; snake bite; scorpion } \\
\text { bite; boils; blisters; wounds; asthma; inflammations; cuts; } \\
\text { febrifuge }\end{array}$ & General & $\begin{array}{l}\text { Ignacimuthu et al. 2008, Jain et } \\
\text { al. 2009, Rao et al. } 2006\end{array}$ \\
\hline
\end{tabular}

Published 11 February 2019

http://dx.doi.org/10.32859/era.18.4.1-112 


\begin{tabular}{|c|c|c|c|c|c|}
\hline \multicolumn{6}{|l|}{ Clusiaceae } \\
\hline $\begin{array}{l}\text { Agasthiyamalaia pauciflora (Bedd.) S. } \\
\text { Rajkumar and Janarth. }\end{array}$ & Tree & - & Infectious diseases & General & Ghanthi and Manickam 2008 \\
\hline Calophyllum apetalum Willd. & Tree & Bark & Resin & General & Jayakumar et al. 2010 \\
\hline Calophyllum inophyllum L. & Tree & $\begin{array}{l}\text { Leaves, } \\
\text { seed, fruit, } \\
\text { bark }\end{array}$ & $\begin{array}{l}\text { Antibacterial; Antitumor; cytotoxic; antibacterial; analgesic; } \\
\text { scabies; stomach ache; edible }\end{array}$ & General & $\begin{array}{l}\text { Bhat et al. 2014, Rao et al. } \\
\text { 2006, Valsaraj et al. } 1997\end{array}$ \\
\hline Garcinia cowa Roxb. ex Choisy & Tree & Fruit & Laxative & General & Prasad et al. 2008 \\
\hline Garcinia gummi-gutta (L.) Roxb. & Tree & $\begin{array}{l}\text { Leaves, } \\
\text { fruit, stem }\end{array}$ & $\begin{array}{l}\text { Dysentery; diarrhea; antibacterial; pimples; boils; eczema; } \\
\text { cholesterol; souring agent }\end{array}$ & General & $\begin{array}{l}\text { Prasad et al. 2013, Valsaraj et } \\
\text { al. } 1997\end{array}$ \\
\hline Garcinia indica (Thouars) Choisy & Tree & Leaves, fruit & Cosmetic ingredients; edible & General & Bhagat et al. 2016 \\
\hline Garcinia talbotii Raizada ex Santapau & Tree & Fruit & Edible & General & Bhagat et al. 2016 \\
\hline $\begin{array}{l}\text { Garcinia xanthochymus Hook. f. ex T. } \\
\text { Anderson }\end{array}$ & Tree & Stem & Muscle pain & General & $\begin{array}{l}\text { Muralidhara Rao and Pullaiah } \\
2007\end{array}$ \\
\hline \multicolumn{6}{|l|}{ Colchicaceae } \\
\hline Colchicum luteum Baker & Herb & Whole plant & Bronchial diseases & General & Kayani et al. 2014 \\
\hline Gloriosa superba L. & Climber & Whole plant & $\begin{array}{l}\text { Asthma; sinusitis; problems during delivery; snake bite; } \\
\text { aphrodisiac; abortion; leprosy; cancer; skin disease; } \\
\text { gonorrhea; rheumatism; labour pain; larvicide; insecticidal; } \\
\text { wormicidal }\end{array}$ & $\begin{array}{l}\text { General; } \\
\text { pregnant } \\
\text { women }\end{array}$ & $\begin{array}{l}\text { Dey and De 2012, Jain 2004, } \\
\text { Lingaraju et al. } 2013\end{array}$ \\
\hline \multicolumn{6}{|l|}{ Combretaceae } \\
\hline $\begin{array}{l}\text { Anogeissus latifolia (Roxb. ex DC.) Wall. } \\
\text { ex Guillem. and Perr. }\end{array}$ & $\begin{array}{l}\text { Medium } \\
\text { sized tree }\end{array}$ & Whole plant & $\begin{array}{l}\text { Get rid of backpain and to cure the damaged tissue; snake } \\
\text { bite; scorpion bite; wound; nausea; insect bite; asthma; } \\
\text { cough; Dysentry; diaherroea; non medicinal purposes; } \\
\text { firewood; liver complaints; cough; aphrodisiac; sores; } \\
\text { stomach ache; }\end{array}$ & $\begin{array}{l}\text { Women; } \\
\text { General }\end{array}$ & $\begin{array}{l}\text { Gairola et al. 2013, Jain et al. } \\
\text { 2004, Reddy et al. 2008, } \\
\text { Sharma et al. } 2013\end{array}$ \\
\hline Combretum albidum G. Don & Climber & Leaves & Stomach ulcers & General & Savithramma et al. 2014 \\
\hline Combretum album Pers. & Climber & $\begin{array}{l}\text { Root, seed, } \\
\text { fruit }\end{array}$ & Antihelmintic to expel parasitic & General & Kanthasamy Kalaichelvi 2016 \\
\hline Combretum latifolium Blume & Climber & Leaves & Diarrhoea & General & Sreeramulu et al. 2013 \\
\hline Getonia floribunda Roxb. & Climber & Whole plant & $\begin{array}{l}\text { Rheumatoid arthritis; astringent; anthelmintic; depurative; } \\
\text { diaphoretic; fever; dysentery; leprosy; worms; jaundice; } \\
\text { ulcers; skin disease; wound; snake bite; fever; jaundice; } \\
\text { ringworm }\end{array}$ & General & $\begin{array}{l}\text { Murthy et al. 2008, Kshirsagar } \\
\text { and Singh 2001, Kumar et al. } \\
\text { 2007, Xavier et al. } 2014\end{array}$ \\
\hline Lumnitzera racemosa Willd. & $\begin{array}{l}\text { Shrub to } \\
\text { small tree }\end{array}$ & Whole plant & - & - & Dey and De 2012 \\
\hline Terminalia alata Wall. & Tree & Bark, leaves & Wounds; epilepsy & General & Dubey et al. 2012 \\
\hline $\begin{array}{l}\text { Terminalia arjuna (Roxb. ex DC.) Wight } \\
\text { and Arn. }\end{array}$ & Tree & $\begin{array}{l}\text { Bark, fruit, } \\
\text { root, leaves }\end{array}$ & $\begin{array}{l}\text { Abortifacient; abdominal pain; cardiac problems; } \\
\text { dysentery; influenza; gynaecological disorders; heart } \\
\text { diseases; dyspepsia; boils; blisters; wound; snakebite; } \\
\text { urinary problems; skin diseases; earache }\end{array}$ & $\begin{array}{l}\text { General; } \\
\text { pregnant } \\
\text { women; } \\
\text { women }\end{array}$ & $\begin{array}{l}\text { Ignacimuthu et al. } 2008, \\
\text { Kosalge and Fursule } 2009 \text {, } \\
\text { Reddy et al. } 2008\end{array}$ \\
\hline Terminalia bellirica (Gaertn.) Roxb. & Tree & $\begin{array}{l}\text { Fruit, stem } \\
\text { bark, } \\
\text { exocarp, }\end{array}$ & $\begin{array}{l}\text { Asthma; antibacterial; contraceptive; leucoderma; } \\
\text { diarrhoea; skin diseases; blisters; laxative; leprosy; eye } \\
\text { diseases; skin burns; stomach problems; diabetes; } \\
\text { infertility; fever; gout }\end{array}$ & General & $\begin{array}{l}\text { Das et al. 2014, Kumar et al. } \\
2011, \text { Valsaraj et al. } 1997\end{array}$ \\
\hline
\end{tabular}

Published 11 February 2019

http://dx.doi.org/10.32859/era.18.4.1-112 


\begin{tabular}{|c|c|c|c|c|c|}
\hline & & $\begin{array}{l}\text { seed, bark, } \\
\text { leaves }\end{array}$ & & & \\
\hline Terminalia bialata (Roxb.) Steud. & Tree & Bark & Rheumatism & General & Prasad et al. 2008 \\
\hline Terminalia catappa L. & Tree & $\begin{array}{l}\text { Leaves, } \\
\text { Fruit, gum }\end{array}$ & Cough; dysentry; liver diseases; prevents cancer & General & $\begin{array}{l}\text { Ayyanar and Ignacimuthu 2010, } \\
\text { Suganthi and Libina } 2015\end{array}$ \\
\hline Terminalia chebula Retz. & Tree & Whole plant & $\begin{array}{l}\text { Headache; antibacterial; asthma; indigestion; breast } \\
\text { cancer; cold; cough; contraceptive; fever; constipation; } \\
\text { aphrodisiac; diarrhoea; gastrointestinal disorders; heart } \\
\text { stimulant; acidity; vomiting }\end{array}$ & General & $\begin{array}{l}\text { Ayyanar 2013, Ayyanar and } \\
\text { lgnacimuthu 2010, Das et al. } \\
2014\end{array}$ \\
\hline Terminalia citrina Roxb. ex Fleming & Tree & Leaves & Wounds & General & Prasad et al. 2008 \\
\hline Terminalia crenulata Roth & Tree & Oil, bark & Dysentery; rheumatic pain; dandruff & General & Kshirsagar and Singh 2001 \\
\hline Terminalia cuneata Roth & Tree & Bark & Itching due to impure blood; bone fracture & General & Bhat et al. 2014 \\
\hline Terminalia glaucescens Planch. ex Benth. & Tree & Leaves & Vegetable & General & Dansi et al. 2008 \\
\hline Terminalia pallida Brandis & Tree & Fruit, root & Inflammation; stomachache; urinary disorders & General & Ram et al. 2004 \\
\hline Terminalia paniculata Roth & Tree & Flower & Cholera & General & Mahishi et al. 2005 \\
\hline Terminalia superba Engl. and Diels & Tree & Leaves & Vegetable & General & Dansi et al. 2008 \\
\hline Terminalia tomentosa Wight and Arn. & Tree & Bark & Intestinal problems & General & Nizar et al. 2015 \\
\hline \multicolumn{6}{|l|}{ Commelinaceae } \\
\hline Amischotolype mollissima (Blume) Hassk. & Herb & Whole plant & $\begin{array}{l}\text { Treating food poisoning; Treating obstetric diseases or } \\
\text { body pain }\end{array}$ & General & Srithi et al. 2009 \\
\hline Commelina benghalensis $\mathrm{L}$. & Herb & Whole plant & $\begin{array}{l}\text { Antiseptic Epilepsy; eyelid infection and skin rash; body } \\
\text { hotness; body pain; edible; helminthiasis; malaria; wound; } \\
\text { leprosy }\end{array}$ & General & Divya and Kalaichelvi \\
\hline Commelina communis L. & Forb/herb & Leaves & Anti-pyretic; anti-inflammatory and diuretic effect. & General & Suganthi and Libina 2015 \\
\hline Commelina erecta L. & $\begin{array}{l}\text { Medium } \\
\text { sized herb }\end{array}$ & Leaves & Sciatica & General & Rao et al. 2011 \\
\hline Commelina longifolia Lam. & Herb & Whole plant & Fever & General & Padal et al. 2010 \\
\hline Cyanotis clavata & Shrub & $\begin{array}{l}\text { Leaves, } \\
\text { roots }\end{array}$ & Cough; biliousness; diarrhea & General & Kanthasamy Kalaichelvi 2016 \\
\hline Cyanotis axillaris (L.) D. Don ex Sweet & Herb & Whole plant & Fever; Abdominal pains & General & Kanthasamy Kalaichelvi 2016 \\
\hline Murdannia edulis (Stokes) Faden & Herb & Root & Aphrodisiac; Spermatorrhoea & $\begin{array}{l}\text { General; } \\
\text { men }\end{array}$ & Malviya et al. 2011 \\
\hline Pollia secundiflora (Blume) Bakh. f. & Herb & $\begin{array}{l}\text { Leaves, } \\
\text { stem }\end{array}$ & Leg and stomach swelling & General & Gailea et al. 2016 \\
\hline \multicolumn{6}{|l|}{ Connaraceae } \\
\hline Cnestis ferruginea Vahl ex DC. & Shrub & $\begin{array}{l}\text { Bark, } \\
\text { leaves, sap, } \\
\text { fruit }\end{array}$ & $\begin{array}{l}\text { Periodontitis; headache; bronchitis; eye troubles; } \\
\text { dysmenorrhoea; all manner of pains; migraine; sinusitis; } \\
\text { toothache; conjunctivitis; anti-stress }\end{array}$ & General & Ishola et al. 2011 \\
\hline \multicolumn{6}{|l|}{ Convolvulaceae } \\
\hline Argyreia cuneata Ker Gawl. & Shrub & Leaves, root & Rheumatism; fever; skin cuts; Anthelmintic; diabetes & General & $\begin{array}{l}\text { Kshirsagar and Singh 2001, } \\
\text { Lingaraju et al. } 2013\end{array}$ \\
\hline Argyreia elliptica Choisy & Climber & Leaves, root & Eye injuries and infections of cattle; stomachache & $\begin{array}{l}\text { General; } \\
\text { cattle }\end{array}$ & Kshirsagar and Singh 2001 \\
\hline Argyreia nervosa (Burm. f.) Bojer & Climber & $\begin{array}{l}\text { Root, } \\
\text { leaves, fruit }\end{array}$ & $\begin{array}{l}\text { Tympany; herpes; leucoderma; blisters; boils; } \\
\text { Hydrocele;Piles;Post-partum problem; edible; tuberculosis }\end{array}$ & General & $\begin{array}{l}\text { Bhagat et al. 2016, Padal et al. } \\
2010, \text { Rao et al. } 2006\end{array}$ \\
\hline
\end{tabular}

Published 11 February 2019

http://dx.doi.org/10.32859/era.18.4.1-112 


\begin{tabular}{|c|c|c|c|c|c|}
\hline Argyreia pomacea Sweet & Climber & Root & $\begin{array}{l}\text { Jaundice; Fever and headache; tuberculosis; acidity; piles; } \\
\text { rheumatism }\end{array}$ & General & Kanthasamy Kalaichelvi 2016 \\
\hline Cuscuta reflexa Roxb. & Climber & Whole plant & $\begin{array}{l}\text { Contraceptive; abortifacient; liver disease; hepatitis; } \\
\text { bronchitis; antioxidant; dandruff; epilepsy; hair fall; piles }\end{array}$ & $\begin{array}{l}\text { General; } \\
\text { pregnant } \\
\text { women }\end{array}$ & $\begin{array}{l}\text { Jain 2004, Murty and Venkaiah } \\
2010, \text { Pushpangadan and Atal } \\
1984\end{array}$ \\
\hline Dichondra repens J.R. Forst. and G. Forst. & Creeper & Leaves & Snake bite & General & Owuor et al. 2005 \\
\hline Evolvulus alsinoides (L.) L. & Herb & Whole plant & $\begin{array}{l}\text { Malaria; veneral diseases; brain disorders; epilepsy; } \\
\text { dysentery; rheumatism; purifies blood; strenghtning of } \\
\text { heart; diarrhoea; syphilis; boils; blisters; fever; improves } \\
\text { memory power; eye sores; increases sperm count; } \\
\text { snakebite; hair growth; jaundice; gynaecological disorders }\end{array}$ & $\begin{array}{l}\text { General; } \\
\text { men; } \\
\text { women }\end{array}$ & $\begin{array}{l}\text { Ayyanar and Ignacimuthu 2005, } \\
\text { Chopda and Mahajan 2009, } \\
\text { Padma et al. 2016, Sharma et } \\
\text { al. } 2013\end{array}$ \\
\hline Evolvulus nummularius (L.) L. & Herb & Whole plant & Fever; burns & General & Padal et al. 2010 \\
\hline Ipomoea alba L. & Herb & \begin{tabular}{|l|} 
Leaves \\
\end{tabular} & Skin diseases & General & Deepak and Gopal 2014 \\
\hline Ipomoea aquatica Forssk. & Herb & $\begin{array}{l}\text { Flower, } \\
\text { shoot, } \\
\text { leaves, root }\end{array}$ & $\begin{array}{l}\text { Diarrhoea; laxative; blood purifier; black ring around the } \\
\text { eye; indigestion; vegetable; hemorrhoids }\end{array}$ & General & $\begin{array}{l}\text { Chander et al. 2015, Dansi et al. } \\
\text { 2008, Prashanth Kumar and } \\
\text { Shiddamallayya 2015, } \\
\text { Shanmugam et al. 2012 }\end{array}$ \\
\hline Ipomoea batatas (L.) Lam. & Herb & $\begin{array}{l}\begin{array}{l}\text { Leaves, } \\
\text { tuber }\end{array} \\
\end{array}$ & Anti diabetic; vegetable & General & Ruba and Mohan 2016 \\
\hline Ipomoea cairica (L.) Sweet & Climber & Whole plant & Fever & General & $\begin{array}{l}\text { Dey and De 2012, Lingaraju et } \\
\text { al. 2013, Padal et al. 2010, } \\
\text { Padal et al. } 2014\end{array}$ \\
\hline Ipomoea carnea Jacq. & Shrub & Whole plant & Tonsils; Neck pain & General & Sreeramulu et al. 2013 \\
\hline Ipomoea cheirophylla O'Donell & Herb & Root & To increase lactation & Women & Sayed et al. 2007 \\
\hline Ipomoea cordifolia Carey ex Voigt & Herb & Root & Skin diseases & General & Kanthasamy Kalaichelvi 2016 \\
\hline Ipomoea eriocarpa R. Br. & Climber & Whole plant & Hysteria; rheumatism; skin diseases; epilepsy; vegetable & General & $\begin{array}{l}\text { Pushpangadan and Atal 1984, } \\
\text { Sharma et al. } 2013\end{array}$ \\
\hline Ipomoea fistulosa Mart. ex Choisy & Shrub & Leaves & dogbite & General & Sharma et al. 2010 \\
\hline Ipomoea hederifolia L. & Climber & Root, leaves & Cataract; tonic & General & Rao et al. 2011 \\
\hline Ipomoea lacunosa L. & Herb & Leaves & Bone fractures & General & Bosco and Arumugam 2012 \\
\hline Ipomoea marginata (Desr.) Verdc. & $\begin{array}{l}\text { Twining } \\
\text { herb }\end{array}$ & Root, leaves & Snake bite (viper); intestinal motility enhancing property & General & Majumder et al. 2013 \\
\hline Ipomoea mauritiana Jacq. & Climber & Root & Diabetes; sexually transmitted diseases; vegetable & General & Dansi et al. 2008 \\
\hline Ipomoea obscura (L.) Ker Gawl. & $\begin{array}{l}\text { Twining } \\
\text { herb }\end{array}$ & Whole plant & $\begin{array}{l}\text { Stomach ulcer; cough; asthma; cold; pain; induces } \\
\text { conception; rheumatoid arthritis; wound; sprain; } \\
\text { stomachache }\end{array}$ & General & $\begin{array}{l}\text { Ayyanar and Ignacimuthu } 2005, \\
\text { Ignacimuthu et al. } 2008\end{array}$ \\
\hline Ipomoea pes-caprae (L.) R. Br. & Herb & Leaves & Rheumatism & General & Sarvalingam et al. 2011 \\
\hline Ipomoea pes-tigridis $\mathrm{L}$. & Climber & $\begin{array}{l}\text { Leaves, } \\
\text { seed, root }\end{array}$ & Antidote; dog bites; boils; pimples; wound & General & $\begin{array}{l}\text { Dey and De 2012, Sarvalingam } \\
\text { et al. } 2011\end{array}$ \\
\hline Ipomoea staphylina Roem. and Schult. & $\begin{array}{l}\text { Straggling } \\
\text { herb }\end{array}$ & $\begin{array}{l}\text { Leaves, } \\
\text { latex }\end{array}$ & Edema; joint pain; foot cracks & General & Sarvalingam et al. 2011 \\
\hline Ipomoea triloba L. & Climber & Leaves & Vegetable & General & Dansi et al. 2008 \\
\hline Ipomoea turbinata Lag. ex Choisy & Herb & Leaves & Constipation & General & Sreeramulu et al. 2013 \\
\hline
\end{tabular}

Published 11 February 2019

http://dx.doi.org/10.32859/era.18.4.1-112 


\begin{tabular}{|c|c|c|c|c|c|}
\hline Ipomoea vagans Baker & $\begin{array}{l}\text { Trailing or } \\
\text { semi erect } \\
\text { herb }\end{array}$ & Leaves & Vegetable & General & Dansi et al. 2008 \\
\hline $\begin{array}{l}\text { Jacquemontia paniculata (Burm. f.) Hallier } \\
\text { f. }\end{array}$ & Climber & Root & - & General & Chopda and Mahajan 2009 \\
\hline Jacquemontia tamnifolia (L.) Griseb. & Herb & Leaves & Vegetable & General & Dansi et al. 2008 \\
\hline $\begin{array}{l}\text { Lepistemon owariense (P. Beauv.) Hallier } \\
\text { f. }\end{array}$ & Herb & Leaves & Vegetable & General & Dansi et al. 2008 \\
\hline Merremia aegyptia (L.) Urb. & Climber & Leaves & Fever; cough; jaundice & General & Kanthasamy Kalaichelvi 2016 \\
\hline Merremia emarginata (Burm. f.) Hallier f. & Herb & Whole plant & Boils; blisters; cuts; cough; cold & General & $\begin{array}{l}\text { Shanmugam et al. 2012, } \\
\text { Sreeramulu et al. } 2013\end{array}$ \\
\hline Merremia gangetica Cufod. & Herb & Root & Snake bite & General & Naidu et al. 2013 \\
\hline Merremia hederacea (Burm. f.) Hallier f. & Climber & Whole plant & Hair tonic; oral health care & General & Hebbar et al. 2004 \\
\hline Merremia tridentata (L.) Hallier $\mathrm{f}$. & Herb & Whole plant & $\begin{array}{l}\text { Intestinal worms; constipation; hair growth; rheumatism; } \\
\text { piles; urinary disorders; diabetes; tooth problems }\end{array}$ & General & $\begin{array}{l}\text { Rao et al. 2006, Shanmugam et } \\
\text { al. } 2012\end{array}$ \\
\hline Operculina turpethum (L.) Silva Manso & Climber & Whole plant & Fever; earache & General & Chopda and Mahajan 2009 \\
\hline Porana paniculata Roxb. & $\begin{array}{l}\text { Twining } \\
\text { shrub }\end{array}$ & $\begin{array}{l}\text { Leaves, } \\
\text { bark }\end{array}$ & Jaundice & $\begin{array}{l}\text { Women; } \\
\text { new born } \\
\text { child }\end{array}$ & Jain et al. 2004 \\
\hline Rivea hypocrateriformis Choisy & Climber & $\begin{array}{l}\text { Stem, } \\
\text { leaves, resin }\end{array}$ & $\begin{array}{l}\text { Diarrhoea; tooth-ache; piles; cough; headache; fever; skin } \\
\text { diseases }\end{array}$ & General & $\begin{array}{l}\text { Rao et al. 2006, Sarvalingam et } \\
\text { al. } 2011\end{array}$ \\
\hline \multicolumn{6}{|l|}{ Cornaceae } \\
\hline Alangium salviifolium (L.f.) Wangerin & Tree & $\begin{array}{l}\text { Fruit, root, } \\
\text { bark }\end{array}$ & $\begin{array}{l}\text { Bone fractures; diabetes; snake bite; oedema; labour } \\
\text { pains; leucorrhea; anthelmintic; boils; blisters; wounds; } \\
\text { skin disease; paralysis }\end{array}$ & General & $\begin{array}{l}\text { Madhu and Suvartha 2009, } \\
\text { Ratnam and Raju 2008, Samy et } \\
\text { al. } 2008\end{array}$ \\
\hline \multicolumn{6}{|l|}{ Costaceae } \\
\hline $\begin{array}{l}\text { Cheilocostus speciosus (J. Koenig) C.D. } \\
\text { Specht }\end{array}$ & Herb & Whole plant & $\begin{array}{l}\text { Anti-inflammatory; anthelmintic; antifungal; treatment of } \\
\text { rheumatism; diabetes; fever; respiratory tract illnesses; } \\
\text { gastrointestinal disorders; leprosy; infertility; pain; urinary } \\
\text { disorders; helminthiasis; eye and ear infections; hepatic } \\
\text { problems; sexually transmitted diseases; snake bite; } \\
\text { tumor; constipation; stomachache; ulcers; abortion; } \\
\text { diabetes; asthma; malaria }\end{array}$ & General & $\begin{array}{l}\text { Bosco and Arumugam 2012, } \\
\text { Padal et al. } 2014, \text { Ramya 2008, } \\
\text { Rao et al. } 2006\end{array}$ \\
\hline Costus pictus D. Don & Herb & - & Diabetes & General & Chithra et al. 2016 \\
\hline \multicolumn{6}{|l|}{ Crassulaceae } \\
\hline Bryophyllum pinnatum (Lam.) Oken & Herb & Whole plant & $\begin{array}{l}\text { Malaria; stomach ulcer; skin burn; burning sensational } \\
\text { urination; scabies; leucoderma; boils; kidney stone; } \\
\text { eczema; pruritus; snake bite }\end{array}$ & General & $\begin{array}{l}\text { Manikandan 2005, Sharma et al. } \\
2013\end{array}$ \\
\hline Kalanchoe laciniata (L.) DC. & Herb & Leaves & Tonic; post-labor tonic; skin diseases & $\begin{array}{l}\text { General; } \\
\text { women }\end{array}$ & Srithi et al. 2009 \\
\hline Sedum roseum (L.) Scop. & Herb & Whole plant & Cognitive improvement; anti- aging; altitude sickness & General & Acharya and Kaphle 2015 \\
\hline \multicolumn{6}{|l|}{ Cucurbitaceae } \\
\hline Cayaponia laciniosa (L.) C. Jeffrey & Climber & - & Impotency & $\begin{array}{l}\text { Men; } \\
\text { women }\end{array}$ & Malviya et al. 2011 \\
\hline Ceratosanthes palmata (L.) Urb. & Climber & Fruit & Acute colic or stomachache. & General & Ignacimuthu et al. 2008 \\
\hline
\end{tabular}

Published 11 February 2019

http://dx.doi.org/10.32859/era.18.4.1-112 


\begin{tabular}{|c|c|c|c|c|c|}
\hline Citrullus colocynthis (L.) Schrad. & Climber & Whole plant & $\begin{array}{l}\text { Purgative; jaundice; rheumatism; snake bite; scorpion } \\
\text { sting; bowel complaints; epilepsy; inflammation of breasts; } \\
\text { hair growth; malaria; hepatitis; hysteria }\end{array}$ & $\begin{array}{l}\text { General; } \\
\text { women; } \\
\text { cow; goat }\end{array}$ & $\begin{array}{l}\text { Chopda and Mahajan 2009, } \\
\text { Dansi et al. 2008, Jain et al. } \\
2009\end{array}$ \\
\hline $\begin{array}{l}\text { Citrullus lanatus (Thunb.) Matsum. and } \\
\text { Nakai }\end{array}$ & Climber & Fruit, leaves & Cooling agent; vegetable & General & Dansi et al. 2008 \\
\hline Coccinia grandis (L.) Voigt & Climber & Whole plant & $\begin{array}{l}\text { Diabetes; ulcer; ear pain; scorpion sting; Dysentery; } \\
\text { Tympany; Boils; Blisters; Cuts; dysuria; Skin diseases and } \\
\text { bronchitis; antidote; diabetes; gastrointestinal disorders }\end{array}$ & General & $\begin{array}{l}\text { Bhat et al. 2014, Chopda and } \\
\text { Mahajan 2009, Kadhirvel et al. } \\
\text { 2010, Kshirsagar and Singh } \\
\text { 2001, Pradheeps and Poyyamoli } \\
2013\end{array}$ \\
\hline Corallocarpus epigaeus (Rottler) Hook. f. & Climber & $\begin{array}{l}\text { Root, tuber, } \\
\text { rhizome, } \\
\text { fruit }\end{array}$ & $\begin{array}{l}\text { Joint pains; typhoid; diabetes; rheumatism; leprosy; } \\
\text { headache; dysentery; edible }\end{array}$ & General & $\begin{array}{l}\text { Basha et al. 2011, Ignacimuthu } \\
\text { et al. } 2008\end{array}$ \\
\hline Cucumeropsis mannii Naudin & Climber & Leaves & Vegetable & General & Dansi et al. 2008 \\
\hline $\begin{array}{l}\text { Cucumis leiospermus (Wight and Arn.) } \\
\text { Ghebret. and Thulin }\end{array}$ & Climber & Fruit & Edible & General & Bhagat et al. 2016 \\
\hline Cucumis melo L. & Climber & Root, leaves & Purgative; snake bite; less irritation; stomachache & General & Kanthasamy Kalaichelvi 2016 \\
\hline Cucumis prophetarum L. & Climber & Fruit & Emetic & General & Lingaraju et al. 2013 \\
\hline Cucumis sativus $\mathrm{L}$. & Climber & Fruit & Antimalaria & General & Gohain et al. 2015 \\
\hline Cucurbita maxima Duchesne & Climber & Aerial parts & Inflammations; burns; vegetable & General & $\begin{array}{l}\text { Dansi et al. 2008, Prashanth } \\
\text { Kumar and Shiddamallayya } \\
2015\end{array}$ \\
\hline Cucurbita moschata Duchesne & Climber & Leaves & Vegetable & General & Dansi et al. 2008 \\
\hline Cucurbita pepo L. & Climber & Leaves & Vegetable & General & Dansi et al. 2008 \\
\hline Diplocyclos palmatus (L.) C. Jeffrey & Climber & Whole plant & $\begin{array}{l}\text { Body pain; dysentery; cough; rheumatic pain; fever; } \\
\text { ovulation enhancement; headache; fertility; taken daily for } \\
7 \text { days before ovulation period for conceiving a male child }\end{array}$ & $\begin{array}{l}\text { General; } \\
\text { women }\end{array}$ & $\begin{array}{l}\text { Ignacimuthu et al. 2008, Padal } \\
\text { et al. } 2010, \text { Pushpangadan and } \\
\text { Atal } 1984\end{array}$ \\
\hline Hodgsonia macrocarpa (Blume) Cogn. & Climber & Root, seed & Typhoid & General & Porte 2014 \\
\hline Kedrostis foetidissima (Jacq.) Cogn. & Climber & Root, leaves & Dermatitis; eczema; stomach problems & General & Sarvalingam et al. 2011 \\
\hline Lagenaria breviflora (Benth.) Roberty & Climber & Leaves & Vegetable & General & Dansi et al. 2008 \\
\hline Lagenaria siceraria (Molina) Standl. & Climber & $\begin{array}{l}\text { Leaves, } \\
\text { root, flower, } \\
\text { fruit }\end{array}$ & $\begin{array}{l}\text { Liver diseases; constipation; foot; mouth disease; reduce } \\
\text { baldness; headache; antidiabetic; anthelmintic }\end{array}$ & $\begin{array}{l}\text { General; } \\
\text { cattle }\end{array}$ & Dansi et al. 2008 \\
\hline Luffa acutangula (L.) Roxb. & Climber & $\begin{array}{l}\text { Fruit, } \\
\text { leaves, } \\
\text { tendril, seed }\end{array}$ & Liver disease; snakebite; jaundice; vegetable & General & $\begin{array}{l}\text { Bhagat et al. 2016, Dey and De } \\
2012\end{array}$ \\
\hline Luffa cylindrica (L.) M. Roem. & Climber & Leaves & Indigestion; abdominal pain; vegetable & General & $\begin{array}{l}\text { Chander et al. 2015, Dansi et al. } \\
2008\end{array}$ \\
\hline Luffa echinata Roxb. & Climber & Fruit & Liver diseases & General & Jain 2004 \\
\hline Momordica balsamina L. & Climber & $\begin{array}{l}\text { Seed, fruit, } \\
\text { leaves }\end{array}$ & $\begin{array}{l}\text { Anthelmintic; fever and extreme uterine bleeding; to treat } \\
\text { syphilis; rheumatism; hepatitis; and skin disorders; } \\
\text { stomach and intestinal complaints; to help the mother to } \\
\text { regenerate her lost blood during labor and to purify her } \\
\text { breast milk }\end{array}$ & $\begin{array}{l}\text { General; } \\
\text { women }\end{array}$ & Bharathi and John 2013 \\
\hline
\end{tabular}

Published 11 February 2019

http://dx.doi.org/10.32859/era.18.4.1-112 


\begin{tabular}{|c|c|c|c|c|c|}
\hline Momordica charantia L. & Climber & Whole plant & $\begin{array}{l}\text { Rheumatism; gout; piles; leprosy; disease of the liver and } \\
\text { spleen; diabetes; sores; infections; hepatitis; fever; } \\
\text { measles; malaria; menstural problems; jaundice; } \\
\text { abortifacient; skin disease; vermifuge; snake bite; chest } \\
\text { pain(children); piles; contraceptive; Post-natal complaints; } \\
\text { anthelmintic; increases lactation }\end{array}$ & $\begin{array}{l}\text { General; } \\
\text { children }\end{array}$ & $\begin{array}{l}\text { Dansi et al. 2008, Ignacimuthu } \\
\text { et al. 2008, Joseph and Antony } \\
2008 \text {, Samy et al. } 2008\end{array}$ \\
\hline Momordica cissoides Planch. ex Benth. & Climber & Leaves & Vegetable & General & Dansi et al. 2008 \\
\hline $\begin{array}{l}\text { Momordica cochinchinensis (Lour.) } \\
\text { Spreng. }\end{array}$ & Climber & $\begin{array}{l}\text { Fruit, seed, } \\
\text { oil }\end{array}$ & $\begin{array}{l}\text { To promote longevity; vitality; and health; to treat infantile } \\
\text { rachitis; xerophthalmia; and night blindness; to improve } \\
\text { growth; liver and spleen disorders; chest complaints; } \\
\text { abdominal pains; dysentery; wounds; hemorrhoids; } \\
\text { bruises; swelling; and pus; for treating anemia and arthritis }\end{array}$ & $\begin{array}{l}\text { General; } \\
\text { children }\end{array}$ & Bharathi and John 2013 \\
\hline Momordica cymbalaria Hook. f. & Climber & Whole plant & $\begin{array}{l}\text { Liver and spleen diseases; abortifacient; diabetes; } \\
\text { rheumatism; ulcer; skin disease; diarrhea; hypoglycemic; } \\
\text { anti implantation; anti-ovulatory; antidiarrhoeal; cancer; } \\
\text { hepatoprotective; antimicrobial; nephroprotective; anti- } \\
\text { ulcer; anticonvulsant; stomachache }\end{array}$ & $\begin{array}{l}\text { General; } \\
\text { women }\end{array}$ & Bharathi and John 2013 \\
\hline Momordica dioica Roxb. ex Willd. & Climber & Whole plant & $\begin{array}{l}\text { Piles; snake bite; cancer; contraceptive; boils; } \\
\text { hypoglycemic; hepatoprotective; chest problems; } \\
\text { antibacterial; intestinal ulcer; migrane; kidney stones; eye } \\
\text { diseases; diarrhoea; fever; antibacteria; malaria; nerve } \\
\text { injuries; antiinflammatory }\end{array}$ & General & $\begin{array}{l}\text { Bhat et al. 2014, Dey and De } \\
\text { 2012, Lingaraju et al. } 2013\end{array}$ \\
\hline $\begin{array}{l}\text { Momordica sahyadrica Kattuk. and V.T. } \\
\text { Antony }\end{array}$ & Climber & $\begin{array}{l}\text { Fruit, tuber, } \\
\text { leaves, twig }\end{array}$ & $\begin{array}{l}\text { Asthmatic and intestinal ulcer; anaemia; anti-inflammatory; } \\
\text { painful eruptions; swellings; breast inflammations; } \\
\text { abortifacient; pain eruptions }\end{array}$ & $\begin{array}{l}\text { General; } \\
\text { women; } \\
\text { pregnant } \\
\text { women; } \\
\text { cows }\end{array}$ & Joseph and Antony 2008 \\
\hline Momordica subangulata Blume & Climber & Aerial parts & Jaundice; edible & General & Bharathi and John 2013 \\
\hline Mukia maderaspatana (L.) M. Roem. & Climber & Leaves, root & Skin-eruption; diabetes & General & Jain et al. 2009 \\
\hline Sechium edule (Jacq.) Sw. & Climber & Fruit & Vitamin C source & General & Devi et al. 2010 \\
\hline Solena amplexicaulis (Lam.) Gandhi & Climber & $\begin{array}{l}\text { Fruit, stem, } \\
\text { root }\end{array}$ & Promotes conception; increases lactation & $\begin{array}{l}\text { General; } \\
\text { women }\end{array}$ & $\begin{array}{l}\text { Bhagat et al. 2016, Ignacimuthu } \\
\text { et al. } 2008\end{array}$ \\
\hline Telfairia occidentalis Hook.f. & Climber & Leaves & Malaria; vegetable & General & $\begin{array}{l}\text { Chenniappan and Kadarkarai } \\
\text { 2010, Dansi et al. } 2008\end{array}$ \\
\hline Trichosanthes cucumerina L. & Climber & Fruit, leaves & Respiratory disorder; skin disease & General & Wagh and Jain 2015 \\
\hline Trichosanthes dioica Roxb. & Climber & $\begin{array}{l}\text { Fruit, } \\
\text { leaves, stem }\end{array}$ & Edible & General & Bhagat et al. 2016 \\
\hline Trichosanthes perrottetiana Cogn. & Climber & Leaves & Flatulence and biliousness & General & Ignacimuthu et al. 2008 \\
\hline Trichosanthes tricuspidata Lour. & Climber & Whole plant & Earache; gonorrhea; snakebite & General & $\begin{array}{l}\text { Dey and De 2012, Padal et al. } \\
2010\end{array}$ \\
\hline $\begin{array}{l}\text { Zehneria maysorensis (Wight and Arn.) } \\
\text { Arn. }\end{array}$ & Climber & Leaves & To kill stomach worms & General & Ayyanar and Ignacimuthu 2005 \\
\hline \multicolumn{6}{|l|}{ Cupressaceae } \\
\hline Cupressus sempervirens L. & Fern/Tree & Fruit & Piles & General & Parada et al. 2009 \\
\hline
\end{tabular}

Published 11 February 2019

http://dx.doi.org/10.32859/era.18.4.1-112 


\begin{tabular}{|c|c|c|c|c|c|}
\hline Juniperus communis $\mathrm{L}$. & $\begin{array}{l}\text { Shrub/ small } \\
\text { tree }\end{array}$ & Fruit & For bad breath & General & Parada et al. 2009 \\
\hline Platycladus orientalis (L.) Franco & Tree & Stem & - & General & Chopda and Mahajan 2009 \\
\hline Thuja occidentalis $\mathrm{L}$. & Tree & Whole plant & Rituals & General & Ruba and Mohan 2016 \\
\hline \multicolumn{6}{|l|}{ Cycadaceae } \\
\hline Cycas beddomei Dyer & Tree & Leaves & Scabies; eye sores & General & Rao et al. 2006 \\
\hline Cycas circinalis L. & Tree & $\begin{array}{l}\text { Bark, seed, } \\
\text { fruit }\end{array}$ & $\begin{array}{l}\text { Swelling; edible; postpartum health; rheumatism; increses } \\
\text { sperm production }\end{array}$ & $\begin{array}{l}\text { General; } \\
\text { men }\end{array}$ & $\begin{array}{l}\text { Das et al. 2013, Udayan et al. } \\
2007\end{array}$ \\
\hline \multicolumn{6}{|l|}{ Cyperaceae } \\
\hline Cyperus javanicus Houtt. & Herb & Root & Anorexia; bone fracture & General & Chander et al. 2015 \\
\hline Cyperus metel L. Pers. & Herb & Root & Malarial treatment & General & Pani et al. 2014 \\
\hline Cyperus rotundus $\mathrm{L}$. & Herb & Whole plant & $\begin{array}{l}\text { Malaria; intestinal colic; pain; indigestion; vomiting; } \\
\text { diarrhoea and flatulence; fever; antibacterial; jaundice; } \\
\text { cough; irregular mensturaton; urinary problems; } \\
\text { gastrointestinal disorders; increase lactation }\end{array}$ & $\begin{array}{l}\text { General; } \\
\text { children; } \\
\text { women }\end{array}$ & $\begin{array}{l}\text { Shanmugam et al. 2012, Zhang } \\
\text { et al. } 2013\end{array}$ \\
\hline Fimbristylis cymosa R.Br. & Herb & Root & Dysentery & General & Shanmugam et al. 2012 \\
\hline Lepironia articulata (Retz.) Domin & Herb & Aerial parts & Treating pain or stimulating appetite & General & Srithi et al. 2009 \\
\hline Rhynchospora colorata (L.) H. Pfeiff. & Herb & $\begin{array}{l}\text { Rhizome, } \\
\text { tuber }\end{array}$ & Diarrhea; diuretic and to cure fever. & General & Jayanthi et al. 2012 \\
\hline Scirpoides holoschoenus (L.) Soják & Herb & Stem & For gingival problems in newborns & \begin{tabular}{|l|} 
Children \\
\end{tabular} & Parada et al. 2009 \\
\hline Scleria corymbosa Roxb. & Herb & Leaves & Blood purifier & General & Sreeramulu et al. 2013 \\
\hline Scleria levis Retz. & Herb & Root & Dysentery & General & Rajendran et al. 2000 \\
\hline \multicolumn{6}{|l|}{ Dennstaedtiaceae } \\
\hline Pteridium aquilinum (L.) Kuhn & Fern & Root & - & General & Chopda and Mahajan 2009 \\
\hline \multicolumn{6}{|l|}{ Dicranaceae } \\
\hline Leucobryum bowringii Mitt. & $\begin{array}{l}\text { Non } \\
\text { vascular }\end{array}$ & - & During body pain & General & Chandra et al. 2016 \\
\hline $\begin{array}{l}\text { Oreas martiana (Hoppe and Hornsch.) } \\
\text { Brid. }\end{array}$ & $\begin{array}{l}\text { Non } \\
\text { vascular }\end{array}$ & - & $\begin{array}{l}\text { Pain; hemostasis; external wounds; epilepsy; } \\
\text { menorrhagia; neurasthenia }\end{array}$ & General & Chandra et al. 2016 \\
\hline \multicolumn{6}{|l|}{ Dilleniaceae } \\
\hline $\begin{array}{l}\text { Dillenia alata (R. Br. ex DC.) Banks ex } \\
\text { Martelli }\end{array}$ & $\begin{array}{l}\text { Non } \\
\text { vascular }\end{array}$ & Leaves & Piles & General & Prabakaran et al. 2013 \\
\hline Dillenia andamanica C.E. Parkinson & $\begin{array}{l}\text { Non } \\
\text { vascular }\end{array}$ & Bark & Leucoderma & General & Prasad et al. 2008 \\
\hline Dillenia indica L. & $\begin{array}{l}\text { Non } \\
\text { vascular }\end{array}$ & $\begin{array}{l}\text { Fruit, bark, } \\
\text { leaves, oil }\end{array}$ & $\begin{array}{l}\text { Abdominal pain; abscess; relieve pain; stimulate appetite; } \\
\text { to prevent dandruff; fever; constipation; dysentery; skin } \\
\text { disease; cough; piles; laxative; malaria }\end{array}$ & $\begin{array}{l}\text { General; } \\
\text { women }\end{array}$ & $\begin{array}{l}\text { Goswami et al. 2016, Prasad et } \\
\text { al. 2008, Rao et al. } 2006\end{array}$ \\
\hline Dillenia pentagyna Roxb. & $\begin{array}{l}\text { Non } \\
\text { vascular }\end{array}$ & $\begin{array}{l}\text { Bark, } \\
\text { leaves, root, } \\
\text { fruit }\end{array}$ & $\begin{array}{l}\text { Rheumatism; dysentery; bone fracture; bronchitis; wound; } \\
\text { piles; diabetes; diarrhoea; delivery; body pain; cuts; burns; } \\
\text { eczema }\end{array}$ & General & $\begin{array}{l}\text { Bhagat et al. 2016, Bhat et al. } \\
\text { 2014, Reddy et al. } 2008\end{array}$ \\
\hline Tetracera sarmentosa (L.) Vahl & $\begin{array}{l}\text { Non } \\
\text { vascular }\end{array}$ & Root & Blood purifier & General & Prasad et al. 2008 \\
\hline
\end{tabular}




\begin{tabular}{|c|c|c|c|c|c|}
\hline Dioscorea alata L. & Climber & Tuber, bulb & $\begin{array}{l}\text { Aphrodisiac; piles; gonorrhea; leprosy; postpartum health; } \\
\text { laxative; vermifuge; gastritis; symptoms of mouth cancer }\end{array}$ & $\begin{array}{l}\text { General; } \\
\text { women }\end{array}$ & $\begin{array}{l}\text { Bhuvaneswari et al. 2015, } \\
\text { Sheikh et al. 2013, Sreeramulu } \\
\text { et al. } 2013\end{array}$ \\
\hline Dioscorea bulbifera L. & Climber & Whole plant & $\begin{array}{l}\text { Indigestion; bone fracture; dysentery; bee stings; } \\
\text { impotency; abdominal pain; contraceptive; abortifacient; } \\
\text { piles; intestinal worms; retention of urine }\end{array}$ & $\begin{array}{l}\text { General; } \\
\text { women; } \\
\text { men }\end{array}$ & $\begin{array}{l}\text { Gairola et al. 2013, Kosalge and } \\
\text { Fursule 2009, Ratnam and Raju } \\
2008\end{array}$ \\
\hline Dioscorea esculenta (Lour.) Burkill & Climber & Tuber & Retention of urine & General & Bhuvaneswari et al. 2015 \\
\hline Dioscorea glabra Roxb. & Climber & Tuber & Edible & General & Sheikh et al. 2013 \\
\hline Dioscorea hispida Dennst. & Climber & Bulb, tuber & Debility; wound & General & $\begin{array}{l}\text { Kosalge and Fursule 2009, } \\
\text { Udayan et al. } 2007\end{array}$ \\
\hline Dioscorea oppositifolia L. & Climber & $\begin{array}{l}\text { Leaves, } \\
\text { flower, } \\
\text { tuber, } \\
\text { rhizome, } \\
\text { root }\end{array}$ & $\begin{array}{l}\text { Snakebite; for increasing body vigour; stomach ulcer; } \\
\text { toothache; aphthae; uterus cancer; fractures; itching; } \\
\text { scorpion bite }\end{array}$ & General & Raju 2008 \\
\hline Dioscorea pentaphylla L. & Climber & $\begin{array}{l}\text { Aerial parts, } \\
\text { root }\end{array}$ & $\begin{array}{l}\text { Rheumatism; Cough; piles; ovulation enhancement; } \\
\text { menorrhegia; rheumatism; intestinal worms; antidiarrhoeal; } \\
\text { increases immunity }\end{array}$ & $\begin{array}{l}\text { General; } \\
\text { women }\end{array}$ & $\begin{array}{l}\text { Sheikh et al. 2013, Tetali et al. } \\
2009\end{array}$ \\
\hline Dioscorea pubera Blume & Climber & $\begin{array}{l}\text { Tuberous } \\
\text { rhizome, } \\
\text { Bulbil }\end{array}$ & ( & General & Sheikh et al. 2013 \\
\hline $\begin{array}{l}\text { Dioscorea tomentosa J. Koenig ex } \\
\text { Spreng. }\end{array}$ & Climber & Tuber & Fever; cough; cold and body swelling; digestion problems & General & $\begin{array}{l}\text { Kanthasamy Kalaichelvi 2016, } \\
\text { Pradheeps and Poyyamoli } 2013\end{array}$ \\
\hline Tacca integrifolia Ker Gawl. & Herb & Rhizome & Contraceptive & General & Das et al. 2014 \\
\hline Tacca leontopetaloides (L.) Kuntze & Herb & $\begin{array}{l}\text { Tuber, } \\
\text { tuber, root }\end{array}$ & Menorrhegia; piles; boils & $\begin{array}{l}\text { General; } \\
\text { women }\end{array}$ & Bhogaonkar and Kadam 2006 \\
\hline \multicolumn{6}{|l|}{ Dipterocarpaceae } \\
\hline Hopea odorata Roxb. & Tree & Bark & Neck Pains & General & Prasad et al. 2008 \\
\hline Shorea robusta Gaertn. & Tree & $\begin{array}{l}\text { Gum, bark, } \\
\text { resin, fruit, } \\
\text { stem, seed }\end{array}$ & $\begin{array}{l}\text { Dysentry; diarrhoea; wound; poor digestion; poor sexual } \\
\text { desire; gonorrhea; skin disease; rheumatic pains; skin } \\
\text { irritation }\end{array}$ & General & $\begin{array}{l}\text { Dey et al. 2012, Gairola et al. } \\
2013\end{array}$ \\
\hline Shorea roxburghii G. Don & Tree & - & Thatching & General & Prabakaran et al. 2013 \\
\hline Shorea tumbuggaia Roxb. & Tree & $\begin{array}{l}\text { Bark, } \\
\text { leaves, resin }\end{array}$ & Stomach ulcers; dysentery; ulcers & General & $\begin{array}{l}\text { Muralidhara Rao and Pullaiah } \\
2007\end{array}$ \\
\hline Vateria indica $\mathrm{L}$. & Tree & Bark & Cough; skin eruption & General & Chopda and Mahajan 2009 \\
\hline \multicolumn{6}{|l|}{ Ditrichaceae } \\
\hline Ditrichum pallidum (Hedw.) Hampe & $\begin{array}{l}\text { Non } \\
\text { vascular }\end{array}$ & - & Used for convulsions & $\begin{array}{l}\text { General; } \\
\text { infants }\end{array}$ & Chandra et al. 2016 \\
\hline \multicolumn{6}{|l|}{ Droseraceae } \\
\hline Drosera burmanni Vahl & Herb & Whole plant & Subjugation; abortion & $\begin{array}{l}\text { General; } \\
\text { men }\end{array}$ & Murty and Venkaiah 2010 \\
\hline \multicolumn{6}{|l|}{ Dryopteridaceae } \\
\hline Dryopteris cochleata (D. Don) C. Chr. & Herb & Rhizome & - & - & Sharma et al. 2013 \\
\hline \multicolumn{6}{|l|}{ Dumortieraceae } \\
\hline Dumortiera hirsuta (Sw.) Nees & Ferns & - & Used as source for antibiotics & General & Chandra et al. 2016 \\
\hline
\end{tabular}

Published 11 February 2019

http://dx.doi.org/10.32859/era.18.4.1-112 


\begin{tabular}{|c|c|c|c|c|c|}
\hline \multicolumn{6}{|l|}{ Ebenaceae } \\
\hline Diospyros candolleana Wight & Tree & Whole plant & Menstrual problems & Women & Prasad et al. 2014 \\
\hline Diospyros chloroxylon Roxb. & Shrub & Flower, root & Snake bite & General & Sharma et al. 2013 \\
\hline Diospyros ebenum J. Koenig ex Retz. & Tree & Whole plant & $\begin{array}{l}\text { Non medicinal commercial purposes; stamina; strengthen } \\
\text { body; body ache }\end{array}$ & General & $\begin{array}{l}\text { Ayyanar and lgnacimuthu 2005, } \\
\text { Rao et al. } 2006\end{array}$ \\
\hline Diospyros malabarica (Desr.) Kostel. & Tree & Bark, fruit & Malarial treatment; edible & General & Bhagat et al. 2016 \\
\hline Diospyros marmorata R. Parker & Tree & Bark & Antiseptic & General & Prasad et al. 2008 \\
\hline Diospyros melanoxylon Roxb. & Tree & $\begin{array}{l}\text { Leaves, } \\
\text { flower, seed, } \\
\text { root, bark, } \\
\text { fruit }\end{array}$ & $\begin{array}{l}\text { Urinary disorders; wound; asthma; bronchitis; chest pain; } \\
\text { antimicrobial; diuretic; skin diseases; boils; diarrhoea; } \\
\text { laxative; anti inflammation }\end{array}$ & General & $\begin{array}{l}\text { Kumar et al. 2007, Rao et al. } \\
2006 \text {, Ratnam and Raju } 2008\end{array}$ \\
\hline Diospyros montana Roxb. & Tree & $\begin{array}{l}\text { Bark, fruit, } \\
\text { leaves }\end{array}$ & $\begin{array}{l}\text { Boils; burns; anorexia; sores; bone fracture; paralysis; joint } \\
\text { pains; wounds; jaundice; fish poisoning }\end{array}$ & General & $\begin{array}{l}\text { Kshirsagar and Singh 2001, } \\
\text { Lingaraju et al. } 2013\end{array}$ \\
\hline Diospyros pyrrhocarpa Miq. & Tree & Leaves & Insect sting & General & Prasad et al. 2008 \\
\hline Diospyros vera (Lour.) A. Chev. & Tree & $\begin{array}{l}\text { Leaves, } \\
\text { fruit, wood }\end{array}$ & Energy stimulant; edible; fire wood & General & Savithramma et al. 2014 \\
\hline \multicolumn{6}{|l|}{ Elaeagnaceae } \\
\hline Elaeagnus angustifolia L. & $\begin{array}{l}\text { Shrub/ small } \\
\text { tree }\end{array}$ & $\begin{array}{l}\text { Fruit, flower, } \\
\text { gum }\end{array}$ & Sore throat & General & Kayani et al. 2014 \\
\hline Elaeagnus conferta Roxb. & Climber & Fruit, leaves & Antidesma; body pain; edible & General & Kanthasamy Kalaichelvi 2016 \\
\hline Elaeagnus latifolia L. & Climber & Fruit & Edible & General & Bhagat et al. 2016 \\
\hline Elaeagnus rhamnoides (L.) A. Nelson & Tree & Fruit & Whooping cough & General & Kayani et al. 2014 \\
\hline Elaeagnus umbellata Thunb. & Shrub & Flower, fruit & Cough and chest pain & General & Kayani et al. 2014 \\
\hline \multicolumn{6}{|l|}{ Elaeocarpaceae } \\
\hline Elaeocarpus lanceifolius Roxb. & Tree & Leaves, root & Malarial fever; cuts; wounds and skin diseases & General & $\begin{array}{l}\text { Muralidhara Rao and Pullaiah } \\
2007\end{array}$ \\
\hline Elaeocarpus oblongus Gaertn. & Tree & Fruit & $\begin{array}{l}\text { Leprosy; pneumonia; rheumatism; ulcers; piles; and } \\
\text { dropsy; theurapetic purpose }\end{array}$ & General & Muthuswamya and Rb 2014 \\
\hline Elaeocarpus serratus L. & Tree & Fruit & Edible & General & Binu 2010 \\
\hline Elaeocarpus tuberculatus Roxb. & Tree & Bark & Antibacterial & General & Valsaraj et al. 1997 \\
\hline \multicolumn{6}{|l|}{ Elatinaceae } \\
\hline Bergia ammannioides Roxb. ex Roth & Undershrub & Root & Stomach-ache & General & Rao et al. 2006 \\
\hline \multicolumn{6}{|l|}{ Entodontaceae } \\
\hline Entodon flavescens (Hook.) A. Jaeger & Moss & - & Earache; cold & General & Chandra et al. 2016 \\
\hline \multicolumn{6}{|l|}{ Ephedraceae } \\
\hline Ephedra gerardiana Wall. Ex Stapf & Shrub & $\begin{array}{l}\text { Leaves, } \\
\text { stem }\end{array}$ & Hay fever; cold; asthma; tuberculosis; bronchitis & General & Kayani et al. 2014 \\
\hline $\begin{array}{l}\text { Ephedra intermedia Schrenk and C.A. } \\
\text { Mey. }\end{array}$ & Shrub & Fruit & Asthma; tuberculosis; chest infections; cough & General & Kayani et al. 2014 \\
\hline Ephedra major Host & Shrub & Stem & - & - & Chopda and Mahajan 2009 \\
\hline \multicolumn{6}{|l|}{ Equisetaceae } \\
\hline Equisetum ramosissimum Desf. & Herb & Aerial parts & Wound healing & General & Latheef et al. 2014 \\
\hline $\begin{array}{l}\text { Hippochaete debilis (Roxb. ex Vaucher) } \\
\text { Ching }\end{array}$ & Herb & Root & Dysentery & General & Rao et al. 2011 \\
\hline
\end{tabular}




\begin{tabular}{|c|c|c|c|c|c|}
\hline \multicolumn{6}{|l|}{ Ericaceae } \\
\hline Gaultheria fragrantissima Wall. & Shrub & Whole plant & Prenatal care and as a health tonic. & $\begin{array}{l}\text { Women, } \\
\text { children }\end{array}$ & Pushpangadan and Atal 1984 \\
\hline Lyonia ovalifolia (Wall.) Drude & Shrub & Seed & Wounds; boils & General & Kumar et al. 2011 \\
\hline Rhododendron anthopogon D. Don & Shrub & $\begin{array}{l}\text { Leaves, } \\
\text { flower }\end{array}$ & High blood pressure & General & Acharya and Kaphle 2015 \\
\hline Rhododendron arboreum Sm. & Shrub & Flower, bark & $\begin{array}{l}\text { Dysentery; diarrhoea; throat clearance when fish bones } \\
\text { get stuck in the gullet; disgestive and respiratory disorders }\end{array}$ & General & $\begin{array}{l}\text { Kumar et al. 2011, Ruba and } \\
\text { Mohan } 2016\end{array}$ \\
\hline Rhododendron lepidotum Wall. ex G. Don & Shrub & $\begin{array}{l}\text { Leaves, } \\
\text { flower }\end{array}$ & Blood purification & General & Acharya and Kaphle 2015 \\
\hline \multicolumn{6}{|l|}{ Erythroxylaceae } \\
\hline Erythroxylum monogynum Roxb. & $\begin{array}{l}\text { Shrub to } \\
\text { small tree }\end{array}$ & $\begin{array}{l}\text { Oil, bark, } \\
\text { fruit }\end{array}$ & Skin disorder; bone fracture; diarrhoea; fire wood & General & Rajan et al. 1997 \\
\hline \multicolumn{6}{|l|}{ Eucommiaceae } \\
\hline Eucommia ulmoides Oliv. & Tree & - & Anti-fungal & General & Zhang et al. 2013 \\
\hline \multicolumn{6}{|l|}{ Euphorbiaceae } \\
\hline Acalypha alnifolia Klein ex Willd. & Undershrub & Leaves & Dysentery; fever & General & Rao et al. 2006 \\
\hline Acalypha brachystachya Hornem. & Herb & Whole plant & Cuts and wounds for fast healing and to expell worms & $\begin{array}{l}\text { Children; } \\
\text { General }\end{array}$ & Pushpangadan and Atal 1984 \\
\hline Acalypha fruticosa Forssk. & Shrub & Whole plant & $\begin{array}{l}\text { Febrifuge; gonorrhoea; whooping cough; constipation; eye } \\
\text { infection; tooth ache; wound; dysentery; reduces breathing } \\
\text { trouble; cold; allergy in lungs; skin disease; to control } \\
\text { worms }\end{array}$ & General & Thirunarayanan 2013 \\
\hline Acalypha indica L. & Herb & Whole plant & $\begin{array}{l}\text { As a laxative and for gastro-intestinal ailments; cough; } \\
\text { snake bite; scorpion sting; scabies; diarrhoea; skin } \\
\text { problems; epilepsy; asthma; bronchitis; cuts; wound; } \\
\text { insect bite; diuretic; tooth and ear ache; dog bite }\end{array}$ & $\begin{array}{l}\text { Children; } \\
\text { General }\end{array}$ & $\begin{array}{l}\text { Ayyanar 2013, Ayyanar and } \\
\text { Ignacimuthu 2011, Bosco and } \\
\text { Arumugam 2012, Ignacimuthu } \\
\text { 2008, Shanmugam et al. 2009, } \\
\text { Sharma et al. } 2013\end{array}$ \\
\hline Acalypha paniculata Miq. & Herb & Leaves & Loose motion & General & Ramya 2008 \\
\hline Acalypha siamensis Oliv. ex Gage & Shrub & Leaves & To treat malaria and malaria-like symptoms & General & $\begin{array}{l}\text { Chenniappan and Kadarkarai } \\
2010\end{array}$ \\
\hline $\begin{array}{l}\text { Alchornea cordifolia (Schumach. and } \\
\text { Thonn.) Müll. Arg. }\end{array}$ & $\begin{array}{l}\text { Shrub to } \\
\text { small tree }\end{array}$ & Whole plant & Piles & General & Nanjunda 2010 \\
\hline Alysicarpus rugosus (Willd.) DC. & Herb & Root & Scorpion sting & General & Ravishankar 2007 \\
\hline Alysicarpus vaginalis (L.) DC. & Herb & Whole plant & Venereal diseases; antifertility; stomach pain & General & Ayyanar and Ignacimuthu 2005 \\
\hline Aporosa cardiosperma (Gaertn.) Merr. & Tree & Fruit, leaves & Burns treatment & General & Kumar et al. 2007 \\
\hline Astraea lobata (L.) Klotzsch & Herb & Leaves & Vegetable & General & Dansi et al. 2008 \\
\hline $\begin{array}{l}\text { Baliospermum solanifolium (Burm.) } \\
\text { Suresh }\end{array}$ & Shrub & Whole plant & $\begin{array}{l}\text { Treating obstetric diseases or body pain; skin diseases; } \\
\text { body swelling }\end{array}$ & General & $\begin{array}{l}\text { Bhandary et al. 1995, Jain } \\
\text { 2004, Kosalge and Fursule } \\
\text { 2009, Reddy et al. } 2008\end{array}$ \\
\hline Butea monosperma (Lam.) Taub. & $\begin{array}{l}\text { Medium } \\
\text { sized tree }\end{array}$ & $\begin{array}{l}\text { Seed, stem, } \\
\text { bark, gum, } \\
\text { flower, } \\
\text { leaves }\end{array}$ & $\begin{array}{l}\text { Leucorrhoea; stop excessive bleeding after child birth; } \\
\text { ringworm; snake bite; diarrhoea; dysentery; asthma; boils; } \\
\text { wound; abdomen in pain; parasitic infection; rheumatic } \\
\text { swelling; piles; menorrhegia; birth control; abortifacient; }\end{array}$ & $\begin{array}{l}\text { General; } \\
\text { women }\end{array}$ & $\begin{array}{l}\text { Bhat et al. 2014, Gairola et al. } \\
\text { 2013, Jain et al. 2004, Kayani et } \\
\text { al. 2014, Sharma et al. } 2013\end{array}$ \\
\hline
\end{tabular}

Published 11 February 2019

http://dx.doi.org/10.32859/era.18.4.1-112 


\begin{tabular}{|c|c|c|c|c|c|}
\hline & & & $\begin{array}{l}\text { bone fracture; vermifuge; malaria; gynaecological } \\
\text { disorders }\end{array}$ & & \\
\hline Butea superba Roxb. & $\begin{array}{l}\begin{array}{l}\text { Scandent } \\
\text { shrub }\end{array} \\
\end{array}$ & Whole plant & $\begin{array}{l}\text { Piles; stomach; Aphrodisiac pain; jaundice; snake bite; } \\
\text { labour pains; scorpion sting; insect bite; }\end{array}$ & General & $\begin{array}{l}\text { Dey and De 2012, Sreeramulu } \\
\text { et al. } 2013\end{array}$ \\
\hline Cajanus cajan (L.) Millsp. & Subshrub & $\begin{array}{l}\text { Leaves, } \\
\text { seed, fruit }\end{array}$ & $\begin{array}{l}\text { Jaundice; antidiabetic; contraceptive; galactagogus; tooth } \\
\text { ache; vegetable }\end{array}$ & General & $\begin{array}{l}\text { Dansi et al. 2008, Kadhirvel et } \\
\text { al. 2010, Rao et al. } 2006\end{array}$ \\
\hline Cajanus crassus (King) Maesen & Climber & Bark & Dysentry and stomach pain & General & Sharma and Mujundar 2003 \\
\hline Cajanus scarabaeoides (L.) Thouars & Shrub & Whole plant & Skin diseases; contraceptive; dysentery; spermatorrhoea & $\begin{array}{l}\text { General; } \\
\text { men }\end{array}$ & $\begin{array}{l}\text { Malviya et al. 2011, Rao et al. } \\
2006\end{array}$ \\
\hline Calliandra haematocephala Hassk. & Shrub & Leaves & Insecticidal; wormicidal & General & Bhardwaj et al. 2011 \\
\hline Croton argyratus Blume & Tree & Latex & Dental caries & General & Chander et al. 2015 \\
\hline Croton bonplandianus Baill. & Herb & Whole plant & $\begin{array}{l}\text { Scabies; cataract; bronchitis; asthma; itch; wound; cuts; } \\
\text { stops bleeding; joint inflammations }\end{array}$ & General & $\begin{array}{l}\text { Jain et al. } 2009 \text {, Kumar and } \\
\text { Chaturvedi } 2010\end{array}$ \\
\hline Croton malabaricus Bedd. & Tree & Aerial parts & Inflammatory joints and rheumatic arthritis. & General & Pushpangadan and Atal 1984 \\
\hline Croton scabiosus Bedd. & Tree & Bark & Snake bite & General & Padma et al. 2016 \\
\hline Endospermum diadenum (Miq.) Airy Shaw & Tree & Root & Tooth-ache & General & Prasad et al. 2008 \\
\hline Euphorbia antiquorum L. & Shrub & $\begin{array}{l}\text { Root, latex, } \\
\text { stem, fruit }\end{array}$ & $\begin{array}{l}\text { Edema; bone fractures; body pain; gout; anti diabetic; } \\
\text { purgative; nerve disorder; bronchitis; dysentery; } \\
\text { dyspepsia; ulcers; asthma }\end{array}$ & General & Ratnam and Raju 2008 \\
\hline Euphorbia caducifolia Haines & Shrub & Root & Abortifacient & General & Jain et al. 2004 \\
\hline Euphorbia dendroides L. & Shrub & Whole plant & Skin diseases; boils & General & Kshirsagar and Singh 2001 \\
\hline Euphorbia dracunculoides Lam. & Herb & Leaves & 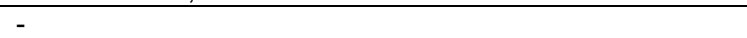 & - & Sharma et al. 2013 \\
\hline $\begin{array}{l}\text { Euphorbia fusiformis Buch. -Ham. ex D. } \\
\text { Don }\end{array}$ & Herb & $\begin{array}{l}\text { Root, } \\
\text { rhizome, } \\
\text { latex, } \\
\text { Leaves, } \\
\text { tuber }\end{array}$ & $\begin{array}{l}\text { Increases secretion of mother's milk; joint pain and } \\
\text { rheumatism; diarrhea; cracks; skin diseases; headache; } \\
\text { liver disorder; fever }\end{array}$ & General & $\begin{array}{l}\text { Anusuya et al. 2010, Wagh anc } \\
\text { Jain } 2015\end{array}$ \\
\hline Euphorbia heterophylla L. & Herb & Whole plant & $\begin{array}{l}\text { Diarrhoea; dysentery; urinary disorders; itches; } \\
\text { gonorrhoea; improves lactation in cattle; wound; skin } \\
\text { diseases }\end{array}$ & $\begin{array}{l}\text { General; } \\
\text { cattle }\end{array}$ & $\begin{array}{l}\text { Kumar and Chaturvedi 2010, } \\
\text { Sharma and Mujundar } 2003\end{array}$ \\
\hline Euphorbia hirta L. & Herb & Whole plant & $\begin{array}{l}\text { Asthma; respiratory infections; stomachache; wounds; } \\
\text { cough; dysentery; snakebite; jaundice; warts removal; } \\
\text { scabies; burns; antiseptic; diarrhoea; pimples; leucorrhea; } \\
\text { nerve bleeding; sores; gastric troubles; increases lactation; } \\
\text { malaria; skin boils; blisters; cuts }\end{array}$ & General & $\begin{array}{l}\text { Jain 2004, Samy et al. 2008, } \\
\text { Sreeramulu et al. } 2013\end{array}$ \\
\hline Euphorbia meenae S. Carter & Herb & Leaves & Boils; Blisters; Cuts & General & Sreeramulu et al. 2013 \\
\hline Euphorbia neriifolia L. & $\begin{array}{l}\text { Shrub to } \\
\text { small tree }\end{array}$ & $\begin{array}{l}\text { Leaves, } \\
\text { stem, latex, } \\
\text { root, bark }\end{array}$ & $\begin{array}{l}\text { Abotifacient; cough; joint pain; burns; bone fracture; } \\
\text { earache; liver diseases }\end{array}$ & $\begin{array}{l}\text { General; } \\
\text { children; } \\
\text { pregnant } \\
\text { women }\end{array}$ & $\begin{array}{l}\text { Jain et al. 2004, Namsa et al. } \\
2009\end{array}$ \\
\hline Euphorbia nivulia Buch. -Ham. & Shrub & Stem, latex & Cure bone fractures; antiseptic & General & Kumar and Chaturvedi 2010 \\
\hline Euphorbia perbracteata Gage & Herb & Whole plant & Insecticide & General & Kumar and Chaturvedi 2010 \\
\hline Euphorbia pilosa L. & Herb & Latex & - & - & Chopda and Mahajan 2009 \\
\hline Euphorbia pulcherrima Willd. ex Klotzsch & Undershrub & Whole plant & $\begin{array}{l}\text { Cure rhuematic pain; Relieve joint pains; antiinflammatory } \\
\text { agent; }\end{array}$ & General & Kumar and Chaturvedi 2010 \\
\hline
\end{tabular}

Published 11 February 2019

http://dx.doi.org/10.32859/era.18.4.1-112 


\begin{tabular}{|c|c|c|c|c|c|}
\hline Euphorbia serpens Kunth & Climber & Leaves & Anticancer; antimicrobial & General & Dhivya and Kalaichelvi 2015 \\
\hline Euphorbia thymifolia L. & Herb & Whole plant & $\begin{array}{l}\text { Snake bite; astringent; anathelmatic; ring worm; laxative; } \\
\text { intestinal inflammation; dysentery; diarrhoea; indigestion; } \\
\text { purgative }\end{array}$ & $\begin{array}{l}\text { General; } \\
\text { children; } \\
\text { infants }\end{array}$ & $\begin{array}{l}\text { Chopda and Mahajan 2009, } \\
\text { Pushpangadan and Atal } 1984\end{array}$ \\
\hline Euphorbia tirucalli L. & $\begin{array}{l}\text { Shrub to } \\
\text { small tree }\end{array}$ & $\begin{array}{l}\text { Stem, } \\
\text { leaves, } \\
\text { latex, bark, } \\
\text { twigs }\end{array}$ & $\begin{array}{l}\text { Remove warts; skin diseases; fish poison; rheumatism; } \\
\text { bone fractures; migrane; headache; abortion }\end{array}$ & $\begin{array}{l}\text { General; } \\
\text { pregnant } \\
\text { women }\end{array}$ & $\begin{array}{l}\text { Ratnam and Raju 2008, } \\
\text { Sreeramulu et al. } 2013\end{array}$ \\
\hline Euphorbia tithymaloides L. & $\begin{array}{l}\text { Succulent } \\
\text { shrub }\end{array}$ & Whole plant & Cure headache; skin disease; urinary problems; snakebite & General & $\begin{array}{l}\text { Dey and De 2012, Kumar and } \\
\text { Chaturvedi } 2010\end{array}$ \\
\hline Excoecaria agallocha $\mathrm{L}$. & Tree & $\begin{array}{l}\text { Flower, root, } \\
\text { oil }\end{array}$ & $\begin{array}{l}\text { Eye diseases; Tooth ache; intestinal worms; Joint pain; } \\
\text { leprosy }\end{array}$ & General & Sivalingam et al. 2016 \\
\hline Falconeria insignis Royle & Tree & Latex & - & General & Sharma et al. 2013 \\
\hline Givotia moluccana (L.) Sreem. & Tree & Bark, fruit & Breathing problems; diabetes & General & Pradheeps and Poyyamoli 2013 \\
\hline Homonoia riparia Lour. & Shrub & $\begin{array}{l}\text { Leaves, } \\
\text { bark }\end{array}$ & Skin bulging; blood clotting; muscle fracture & General & Rajendran et al. 2012 \\
\hline Jatropha curcas L. & Shrub & Whole plant & $\begin{array}{l}\text { Arthritis; pain; inflammation; itched and blistered skin; } \\
\text { toothache; purgative; earache; mouth ulcer; tumors; } \\
\text { digestion; wound; snakebite; mouth foetid smell; scabies; } \\
\text { bio diesel; malaria; cold; fever; antiseptic; aphrodisiac; } \\
\text { boils; skin diseases; insecticidal; wormicidal; fish } \\
\text { poisoning; chronic dysentery; vegetable; jaundice; } \\
\text { constipation; liver troubles; antidiarrhoeal }\end{array}$ & General & Chopda and Mahajan 2009 \\
\hline Jatropha glandulifera Roxb. & Shrub & Latex, tuber & Toothache; gum infection; antidiabetic & General & Hills 2010 \\
\hline Jatropha gossypiifolia L. & Shrub & Whole plant & $\begin{array}{l}\text { Typhoid; jaundice; uterus diseases; skin burns and } \\
\text { irritation; cuts; wormicidal; insecticidal; oral health care; } \\
\text { antibiotic; anticancer }\end{array}$ & Women & $\begin{array}{l}\text { Jain et al. } 2004, \text { Kosalge and } \\
\text { Fursule } 2009\end{array}$ \\
\hline Jatropha multifida L. & Herb & $\begin{array}{l}\text { Leaves, } \\
\text { latex, seed, } \\
\text { exudates }\end{array}$ & Boils; wounds; ulcers; purgative & General & Srithi et al. 2009 \\
\hline Macaranga peltata (Roxb.) Müll. Arg. & Tree & $\begin{array}{l}\text { Gum, } \\
\text { leaves, } \\
\text { stem, wood }\end{array}$ & Sores; boils; wound; stops bleeding; timber & General & Bhat et al. 2014 \\
\hline Mallotus philippensis (Lam.) Müll. Arg. & Tree & Whole plant & $\begin{array}{l}\text { Skin diseases; shivering; leucorrhea; aphrodisiac; skin } \\
\text { burns; hydrocele; stomachache; fire wood }\end{array}$ & General & $\begin{array}{l}\text { Kosalge and Fursule 2009, } \\
\text { Padal et al. } 2014\end{array}$ \\
\hline Manihot carthaginensis (Jacq.) Müll. Arg. & Shrub & Leaves & Vegetable & General & Dansi et al. 2008 \\
\hline Manihot esculenta Crantz & $\begin{array}{l}\text { Shrub to } \\
\text { small tree }\end{array}$ & $\begin{array}{l}\text { Leaves, } \\
\text { flower, } \\
\text { rhizome }\end{array}$ & Vegetable; terminate early pregnancy & $\begin{array}{l}\text { General; } \\
\text { women }\end{array}$ & Arjunan et al. 2016 \\
\hline Micrococca mercurialis (L.) Benth. & Herb & Leaves & Fever; head ache & General & Kanthasamy Kalaichelvi 2016 \\
\hline Ricinus communis $\mathrm{L}$. & Shrub & Whole plant & $\begin{array}{l}\text { Abortifacient; epilepsy; antifertility; body pain; } \\
\text { inflammation; swelling; boils; constipation; dog bite; } \\
\text { dysentery; rheumatism; laxative; skin diseases; typhoid; } \\
\text { arthritis; skin cuts; eye complaints(live stock) }\end{array}$ & $\begin{array}{l}\text { General; } \\
\text { pregnant } \\
\text { women; } \\
\text { veterinary }\end{array}$ & $\begin{array}{l}\text { Jain 2004, Muralidhara Rao and } \\
\text { Pullaiah 2007, Sayed et al. } 2007\end{array}$ \\
\hline Tragia involucrata L. & Herb & Whole plant & Epilepsy; constipation; cough; bronchitis; fever; snakebite & General & Udayan et al. 2007 \\
\hline
\end{tabular}

Published 11 February 2019

http://dx.doi.org/10.32859/era.18.4.1-112 
Ethnobotany Research and Applications

\begin{tabular}{|c|c|c|c|c|c|}
\hline Tragia plukenetii Radcl. -Sm. & $\begin{array}{l}\text { Climbing } \\
\text { shrub }\end{array}$ & Leaves & Scabies & General & Ignacimuthu et al. 2008 \\
\hline \multicolumn{6}{|l|}{ Fabaceae } \\
\hline Abrus precatorius $\mathrm{L}$. & Climber & Whole plant & $\begin{array}{l}\text { Bone fracture; typhoid; Retention of placenta; bronchitis; } \\
\text { fever; leucoderma; rheumatism; skin diseases; veneral } \\
\text { diseases; hair growth; to increase sexual vigour; } \\
\text { abortifacient; abortion; gynaecological disorders; diabetes; } \\
\text { chronic nephritiss; worm infection; gonorrhea; swellings; } \\
\text { paralysis }\end{array}$ & $\begin{array}{l}\text { General; } \\
\text { pregnant } \\
\text { women; } \\
\text { women }\end{array}$ & Upadhya et al. 2012 \\
\hline Abrus pulchellus Thwaites & Climber & Seed, root & Abortifacient; skin diseases & $\begin{array}{l}\text { General; } \\
\text { pregnant } \\
\text { women }\end{array}$ & Prasad et al. 2008 \\
\hline Acacia auriculiformis Benth. & Tree & Seed & Spermicidal and anti HIV properties. & $\begin{array}{l}\text { Men; } \\
\text { General }\end{array}$ & Suganthi and Libina 2015 \\
\hline Acacia caesia (L.) Willd. & Climber & Bark, flower & Wounds healing; skin disease; menstrual problems; & $\begin{array}{l}\text { General; } \\
\text { women }\end{array}$ & Ramya 2008, Rao et al. 2006 \\
\hline Acacia catechu (L.f.) Willd. & Tree & $\begin{array}{l}\text { Gum, bark, } \\
\text { latex, } \\
\text { leaves, fruit, } \\
\text { stem }\end{array}$ & $\begin{array}{l}\text { Expulsion of placenta; abortifacient; dysentery; diarrhoea; } \\
\text { cold; cough; ulcer; chest pain; leprosy }\end{array}$ & $\begin{array}{l}\text { General; } \\
\text { women }\end{array}$ & $\begin{array}{l}\text { Bhat et al. 2012, Bhat et al. } \\
2014 \text {, Jain et al. } 2004\end{array}$ \\
\hline Acacia chundra (Rottler) Willd. & Tree & Whole plant & Asthma; Wounds; Fever; blisters; boils; dysentery & General & $\begin{array}{l}\text { Chopda and Mahajan 2009, } \\
\text { Padal et al. } 2010\end{array}$ \\
\hline Acacia concinna (Willd.) DC. & Tree & Leaves, fruit & Chest pain; fish poison & General & Lingaraju et al. 2013 \\
\hline Acacia farnesiana (L.) Willd. & Tree & $\begin{array}{l}\text { Fruit, bark, } \\
\text { stem, gum }\end{array}$ & $\begin{array}{l}\text { Dog bite; stomach ache; fainting; fever; gastro intestinal } \\
\text { disorders }\end{array}$ & General & $\begin{array}{l}\text { Kshirsagar and Singh 2001, } \\
\text { Sreeramulu et al. } 2013\end{array}$ \\
\hline Acacia ferruginea DC. & Tree & Gum, bark & Fainting; fever; gastro intestinal disorders & General & $\begin{array}{l}\text { Prashanth Kumar and } \\
\text { Shiddamallayya } 2015\end{array}$ \\
\hline Acacia intsia (L.) Willd. & Climber & Bark, fruit & Detergent; edible & General & $\begin{array}{l}\text { Jayakumar et al. 2010, } \\
\text { Prabakaran et al. } 2013\end{array}$ \\
\hline Acacia leucophloea (Roxb.) Willd. & $\begin{array}{l}\text { Moderate } \\
\text { size tree }\end{array}$ & Gum, bark & $\begin{array}{l}\text { Demulcent; to increase sexual potency; tuberculosis; } \\
\text { contraceptive; menstrual complaints; bone fracture; snake } \\
\text { bite; cuts; burms; skin diseases; fever; swellings }\end{array}$ & $\begin{array}{l}\text { Women; } \\
\text { men; } \\
\text { General }\end{array}$ & $\begin{array}{l}\text { Jain et al. 2004, Kshirsagar and } \\
\text { Singh } 2001 \text {, Ramya 2008, Samy } \\
\text { et al. } 2008\end{array}$ \\
\hline Acacia modesta Wall. & $\begin{array}{l}\text { Moderate } \\
\text { size tree }\end{array}$ & $\begin{array}{l}\text { Leaves, } \\
\text { gum, bark, } \\
\text { flower, seed, } \\
\text { stem }\end{array}$ & Cough; respiratory tract problems; tonsillitis & General & Kayani et al. 2014 \\
\hline Acacia nilotica (L.) Delile & $\begin{array}{l}\text { Moderate } \\
\text { size tree }\end{array}$ & $\begin{array}{l}\text { Leaves, } \\
\text { gum, bark, } \\
\text { flower, seed, } \\
\text { pod, stem, } \\
\text { legumes, } \\
\text { fruit }\end{array}$ & $\begin{array}{l}\text { Oralcavity diseases; toothache; asthma; bronchitis; } \\
\text { dysentery; leucorrhea; aphrodisiac; expulsion of placenta; } \\
\text { blood in urine; wound; conjuctivitis; burns; diabetes; snake } \\
\text { bite; headache }\end{array}$ & $\begin{array}{l}\text { General; } \\
\text { women }\end{array}$ & $\begin{array}{l}\text { Arumugam 2012, Bosco and } \\
\text { Kadhirvel et al. 2010, Gairola } \\
\text { 2013, Hebbar et al. 2004, } \\
\text { Kayani et al. } 2014\end{array}$ \\
\hline Acacia pennata (L.) Willd. & Climber & $\begin{array}{l}\text { Leaves, } \\
\text { bark }\end{array}$ & Fits; fever; gastric problems; wound; epilepsy & General & $\begin{array}{l}\text { Kanthasamy Kalaichelvi } 2016 \text {, } \\
\text { Sharma et al. } 2013\end{array}$ \\
\hline Acacia planifrons Wight and Arn. & Tree & Leaves & Skin allergy; cancer & General & Kanthasamy Kalaichelvi 2016 \\
\hline
\end{tabular}

Published 11 February 2019

http://dx.doi.org/10.32859/era.18.4.1-112 
Ethnobotany Research and Applications

\begin{tabular}{|c|c|c|c|c|c|}
\hline Acacia sinuata (Lour.) Merr. & Climber & $\begin{array}{l}\text { Bark, } \\
\text { leaves, fruit }\end{array}$ & Pneumonia in animals; edible; ophthalmic & $\begin{array}{l}\text { General; } \\
\text { animals }\end{array}$ & Kennedy 2006 \\
\hline Acacia torta (Roxb.) Craib & $\begin{array}{l}\text { Rambling } \\
\text { shrub }\end{array}$ & $\begin{array}{l}\text { Stem, bark, } \\
\text { seed, leaves }\end{array}$ & $\begin{array}{l}\text { Cough and dysentery; labour pain; fever; python bite; } \\
\text { stomachache; the treatment of menstrual disorders; } \\
\text { cough; bronchitis }\end{array}$ & $\begin{array}{l}\text { General; } \\
\text { women; } \\
\text { pregnant } \\
\text { women }\end{array}$ & $\begin{array}{l}\text { Kshirsagar and Singh 2001, } \\
\text { Padal et al. 2010, Sreeramulu et } \\
\text { al. } 2013\end{array}$ \\
\hline $\begin{array}{l}\text { Adenanthera microsperma Teijsm. and } \\
\text { Binn. }\end{array}$ & Tree & Bark & Malaria tratement & General & Prasad et al. 2008 \\
\hline Adenanthera pavonina $\mathrm{L}$. & Tree & $\begin{array}{l}\text { Leaves, } \\
\text { seed }\end{array}$ & $\begin{array}{l}\text { Blood pressure; cholera; General paralysis; diarrhoea and } \\
\text { dysentery; rheumatism; toys }\end{array}$ & $\begin{array}{l}\text { General; } \\
\text { children }\end{array}$ & Dhivya and Kalaichelvi 2015 \\
\hline Aeschynomene aspera L. & Shrub & Leaves & Hair fall & General & Das et al. 2013 \\
\hline Aeschynomene indica L. & Herb & Leaves & Snakebite; kidney stones and urinary disorders & General & $\begin{array}{l}\text { Aruna et al. 2012, Dey and De } \\
2012\end{array}$ \\
\hline Albizia amara (Roxb.) B. Boivin & Tree & $\begin{array}{l}\text { Leaves, } \\
\text { bark }\end{array}$ & $\begin{array}{l}\text { Externally affected areas; dandruff; For hair growth; Cuts } \\
\text { and burns; fire wood }\end{array}$ & General & $\begin{array}{l}\text { Arjunan et al. 2016, Dey and De } \\
\text { 2012, Pradheeps and Poyyamoli } \\
2013\end{array}$ \\
\hline Albizia lebbeck (L.) Benth. & Tree & $\begin{array}{l}\text { Bark, } \\
\text { leaves, } \\
\text { seed, seed } \\
\text { oil, resinous } \\
\text { gum, twig, } \\
\text { wood, } \\
\text { flower, stem }\end{array}$ & $\begin{array}{l}\text { Toothache; Antidode; Eye diseases; snake bite; diarrhoea; } \\
\text { wound of rat bite; night blindness; leucoderma; to retard or } \\
\text { slow down aging symptoms; improve complexion; voice } \\
\text { and vitality; leucorrhoea and other venereal diseases in } \\
\text { women; antidote; wheezing protectants; hemorrhoids }\end{array}$ & $\begin{array}{l}\text { Women; } \\
\text { General }\end{array}$ & $\begin{array}{l}\text { Dey and De 2012, Gairola et al. } \\
2013 \text {, Kayani et al. 2014, Padma } \\
\text { et al. } 2016\end{array}$ \\
\hline Albizia odoratissima (L.f.) Benth. & Tree & $\begin{array}{l}\text { Leaves, } \\
\text { bark, wood }\end{array}$ & $\begin{array}{l}\text { Eye diseases; body pains; stomach ache; bronchitis; } \\
\text { diabetes and burning sensation; ulcers; leprosy; skin } \\
\text { diseases; cough }\end{array}$ & General & $\begin{array}{l}\text { Kshirsagar and Singh 2001, } \\
\text { Padal et al. } 2010\end{array}$ \\
\hline Albizia procera (Roxb.) Benth. & Tree & Bark, leaves & Hair growth; dyspepsia; piles; bronchitis & General & $\begin{array}{l}\text { Kayani et al. 2014, Prasad et al. } \\
2008\end{array}$ \\
\hline Bauhinia malabarica Roxb. & Tree & Bark, leaves & Ephemeral fever; Diarrhoea & General & $\begin{array}{l}\text { Lingaraju et al. } 2013, \\
\text { Sreeramulu et al. } 2013\end{array}$ \\
\hline Bauhinia purpurea L. & \begin{tabular}{|l|} 
Moderate \\
size tree
\end{tabular} & $\begin{array}{l}\text { Flower, fruit, } \\
\text { bark }\end{array}$ & $\begin{array}{l}\text { Ulcers; wounds; swollen glands and stomach tumors; } \\
\text { bone fracture; dysentery }\end{array}$ & General & $\begin{array}{l}\text { Ignacimuthu et al. } 2008, \\
\text { Suganthi and Libina } 2015\end{array}$ \\
\hline Bauhinia racemosa Lam. & Small tree & $\begin{array}{l}\text { Bark, flower, } \\
\text { leaves, } \\
\text { legumes, } \\
\text { root }\end{array}$ & Dysentery; diarrhoea; fever; epilepsy; gonorrhea & $\begin{array}{l}\text { General; } \\
\text { veterinary }\end{array}$ & $\begin{array}{l}\text { Padal et al. 2010, Rao et al. } \\
\text { 2006, Reddy et al. 2008, } \\
\text { Sharma et al. } 2013\end{array}$ \\
\hline Bauhinia reticulata DC. & Tree & Leaves & Vegetable & General & Dansi et al. 2008 \\
\hline Bauhinia semla Wunderlin & Tree & Bark & Tonsils; Neck pain & General & Sreeramulu et al. 2013 \\
\hline Bauhinia thonningii Schum. & Shrub/ tree & Leaves & Vegetable & General & Dansi et al. 2008 \\
\hline Bauhinia vahlii Wight and Arn. & Climber & $\begin{array}{l}\text { Seed, root, } \\
\text { leaves }\end{array}$ & $\begin{array}{l}\text { Indigestion; Anthelmintic; drowsiness; uterine stimulant; } \\
\text { dysentery }\end{array}$ & $\begin{array}{l}\text { General; } \\
\text { women }\end{array}$ & $\begin{array}{l}\text { Reddy et al. 2008, Sarvalingam } \\
\text { et al. } 2011\end{array}$ \\
\hline Bauhinia variegata $\mathrm{L}$. & $\begin{array}{l}\text { Moderate } \\
\text { size tree }\end{array}$ & Whole plant & $\begin{array}{l}\text { Diarrhoea; ulcers and leprosy; dysentery; snakebite; skin } \\
\text { infection; piles }\end{array}$ & $\begin{array}{l}\text { General; } \\
\text { infants }\end{array}$ & $\begin{array}{l}\text { Dey and De 2012, Gairola et al. } \\
2013\end{array}$ \\
\hline Caesalpinia bonduc (L.) Roxb. & $\begin{array}{l}\text { Straggling } \\
\text { shrub }\end{array}$ & $\begin{array}{l}\text { Oil, } \\
\text { endosperm, }\end{array}$ & $\begin{array}{l}\text { Pimples; vomiting; bleeding; jaundice; stomach pain; bone } \\
\text { fractures; abortion; diabetes; rheumatism; hydrocele; }\end{array}$ & $\begin{array}{l}\text { women; } \\
\text { General }\end{array}$ & $\begin{array}{l}\text { Chopda and Mahajan 2009, } \\
\text { Dansi et al. 2008, Lingaraju et }\end{array}$ \\
\hline
\end{tabular}

Published 11 February 2019

http://dx.doi.org/10.32859/era.18.4.1-112 


\begin{tabular}{|c|c|c|c|c|c|}
\hline & & $\begin{array}{l}\text { leaves, } \\
\text { seed, fruit }\end{array}$ & $\begin{array}{l}\text { fever; ulcer; acidity; snake bite; vegetable; epilepsy; } \\
\text { Swelling testicles; Anti-rabies; sexual debility; skin disease }\end{array}$ & & $\begin{array}{l}\text { al. 2013, Ratnam and Raju } \\
\text { 2008, Samy et al. } 2008\end{array}$ \\
\hline Caesalpinia coriaria (Jacq.) Willd. & $\begin{array}{l}\text { Medium } \\
\text { sized tree }\end{array}$ & Fruit & Oral health care & General & Hebbar et al. 2004 \\
\hline Caesalpinia crista L. & Climber & Oil, seed & Pimples; scurf; skin diseases & General & Harsha et al. 2003 \\
\hline Caesalpinia decapetala (Roth) Alston & Shrub & - & Antidiarrhoeal & General & Tetali et al. 2009 \\
\hline Caesalpinia mimosoides Lam. & Climber & $\begin{array}{l}\text { Leaves, } \\
\text { tender parts }\end{array}$ & Skin boils & General & $\begin{array}{l}\text { Bhat et al. 2014, Sharma et al. } \\
2013\end{array}$ \\
\hline Caesalpinia pulcherrima (L.) Sw. & Shrub & $\begin{array}{l}\text { Leaves, } \\
\text { bark, seed }\end{array}$ & Abortion; malaria; prolonged labour & $\begin{array}{l}\text { Pregnant } \\
\text { women, } \\
\text { General }\end{array}$ & Murty and Venkaiah 2010 \\
\hline Caesalpinia sappan L. & Tree & Stem, seed & Menstural problems; Blood tonic; post-labor tonic & $\begin{array}{l}\text { Women, } \\
\text { General }\end{array}$ & $\begin{array}{l}\text { Chopda and Mahajan 2009, } \\
\text { Srithi et al. } 2009\end{array}$ \\
\hline Canavalia cathartica Thouars & $\begin{array}{l}\text { Medium } \\
\text { sized } \\
\text { climbing } \\
\text { shrub }\end{array}$ & Root & Venereal diseases & General & Sreeramulu et al. 2013 \\
\hline Canavalia gladiata (Jacq.) DC. & Climber & $\begin{array}{l}\text { Seed, bud, } \\
\text { root, leaves, } \\
\text { legume, } \\
\text { bark }\end{array}$ & $\begin{array}{l}\text { Enlargement of liver; rheumatism; food; edible; epilepsy; } \\
\text { earache }\end{array}$ & General & $\begin{array}{l}\text { Rao et al. 2006, Sreeramulu et } \\
\text { al. } 2013\end{array}$ \\
\hline Cassia fistula L. & $\begin{array}{l}\text { Medium } \\
\text { sized tree }\end{array}$ & $\begin{array}{l}\text { Fruit, stem, } \\
\text { bark, seed, } \\
\text { leaves, root, } \\
\text { wood }\end{array}$ & $\begin{array}{l}\text { Oral ulcers; sore mouth; epilepsy; foetid smell of mouth; } \\
\text { snake bite; dysentery; diarrhoea; tympany; bone fractures; } \\
\text { stomach tumor; purjative; constipation; anthelmintic; insect } \\
\text { bite; constipation; leprosy; chest pain; laxative }\end{array}$ & General & $\begin{array}{l}\text { Bhandary et al. 1995, Bhat et al. } \\
\text { 2014, Ratnam and Raju 2008, } \\
\text { Sharma et al. } 2013\end{array}$ \\
\hline Cassia leschenaultiana DC. & Shrub & $\begin{array}{l}\text { Leaves, } \\
\text { stem }\end{array}$ & $\begin{array}{l}\text { Constipation; cold; fever;intestinal disorder; aguesia and } \\
\text { skin diseases }\end{array}$ & General & Kanthasamy Kalaichelvi 2016 \\
\hline $\begin{array}{l}\text { Chamaecrista absus (L.) H.S. Irwin and } \\
\text { Barneby }\end{array}$ & Herb & $\begin{array}{l}\text { Leaves, } \\
\text { seed }\end{array}$ & $\begin{array}{l}\text { Insecticide; bone fractures; Astringent; cough remedy } \\
\text { Cathartic; skin infections }\end{array}$ & General & $\begin{array}{l}\text { Kanthasamy Kalaichelvi 2016, } \\
\text { Wagh and Jain } 2015\end{array}$ \\
\hline Chamaecrista mimosoides (L.) Greene & Herb & Seed, root & Aphrodisiac; stomachache & General & Savithramma et al. 2014 \\
\hline Cicer arietinum $\mathrm{L}$. & Herb & Seed, stem & Contraceptive; liver diseases & $\begin{array}{l}\text { General; } \\
\text { women }\end{array}$ & Bhogaonkar and Kadam 2006 \\
\hline Clitoria ternatea L. & Slender vine & Whole plant & $\begin{array}{l}\text { Cold; asthma and small pox; syphilis; rheumatism; } \\
\text { stomachache; wounds; indigestion; diabetes and throat } \\
\text { pain; leucoderma; leprosy; hemicrania; amentia; } \\
\text { pulmonary tuberculosis; ophthalmology; insect bites; } \\
\text { snake bites; dysentery; swellings on legs; urinary } \\
\text { disorders; throat pain }\end{array}$ & $\begin{array}{l}\text { Children; } \\
\text { General; } \\
\text { men }\end{array}$ & $\begin{array}{l}\text { Kshirsagar and Singh } 2001 \text {, } \\
\text { Pushpangadan and Atal 1984, } \\
\text { Rao et al. 2006, Xavier et al. } \\
2014\end{array}$ \\
\hline Codariocalyx motorius (Houtt.) H. Ohashi & Shrub & - & Asthma & General & Chithra et al. 2016 \\
\hline Crotalaria albida Roth & Herb & Root & Labour pains & General & Sreeramulu et al. 2013 \\
\hline Crotalaria burhia Benth. & Herb & Whole plant & - & General & Kataria and Kaur 2013 \\
\hline Crotalaria calycina Schrank & Herb & Rhizome & Paronychia & General & Rao et al. 2006 \\
\hline Crotalaria filipes Benth. & Herb & Whole plant & Antibacterial activity & General & Ranjitha et al. 2016 \\
\hline Crotalaria juncea L. & Herb & Flowers & Constipation; blood disorder & General & $\begin{array}{l}\text { Prashanth Kumar and } \\
\text { Shiddamallayya } 2015\end{array}$ \\
\hline
\end{tabular}

Published 11 February 2019

http://dx.doi.org/10.32859/era.18.4.1-112 


\begin{tabular}{|c|c|c|c|c|c|}
\hline Crotalaria laburnifolia L. & Herb & $\begin{array}{l}\text { Root, tuber, } \\
\text { leaves }\end{array}$ & Snake bites; cuts; diarrhoea; throat infections & General & Rao et al. 2006 \\
\hline Crotalaria madurensis Wight and Arn. & Herb & Leaves & Scabies; antibacterial & General & Bhakshu et al. 2008 \\
\hline Crotalaria medicaginea Lam. & Herb & Whole plant & Scabies; impetigo toxaemia & General & Jain et al. 2009 \\
\hline Crotalaria pallida Aiton & Herb & $\begin{array}{l}\text { Leaves, } \\
\text { root, seed }\end{array}$ & Skin diseases; wound; bronchitis; vermifuge; fever & General & Ayyanar and Ignacimuthu 2005 \\
\hline Crotalaria prostrata Willd. & Herb & Leaves & Cuts & General & Kumar et al. 2007 \\
\hline Crotalaria ramosissima Roxb. & Herb & Flower & Eczema & General & Ram et al. 2004 \\
\hline Crotalaria retusa L. & Herb & Whole plant & Cough; fever; eczema; dyspepsia; swelling of the testicle & General & Dhivya and Kalaichelvi 2015 \\
\hline Crotalaria verrucosa $\mathrm{L}$. & Herb & $\begin{array}{l}\text { Leaves, } \\
\text { root, bark, } \\
\text { Seed }\end{array}$ & $\begin{array}{l}\text { Ephemeral fever; Insect bite; Fits; abortion; sores; } \\
\text { toothache; throat infections }\end{array}$ & General & $\begin{array}{l}\text { Rao et al. 2006, Vedavathy et al. } \\
1997\end{array}$ \\
\hline Cullen corylifolium (L.) Medik. & Herb & $\begin{array}{l}\text { Fruit, seed, } \\
\text { oil, roots, } \\
\text { leaves }\end{array}$ & $\begin{array}{l}\text { Leucoderma; antibacterial; skin diseases; frequent } \\
\text { urination; premature ejaculation; impotence; back pains; } \\
\text { promotes sexual vigour; snakebite }\end{array}$ & $\begin{array}{l}\text { General; } \\
\text { men; } \\
\text { women }\end{array}$ & Jain 2004, Chopra et al. 2013 \\
\hline Dalbergia coromandeliana Prain & Shrub & Stem & Antibacterial & General & Edayadulla and Ramesh 2012 \\
\hline Dalbergia lanceolaria L.f. & Tree & $\begin{array}{l}\text { Bark, wood, } \\
\text { leaves, bark }\end{array}$ & Antidiarrhoel; firewood; dysentery; dysmennorhea & General & $\begin{array}{l}\text { Dubey et al. 2012, Mujumdar et } \\
\text { al. } 2005\end{array}$ \\
\hline Dalbergia latifolia Roxb. & Tree & Wood, Bark & Stomachache; skin diseases; lazy grazing animals & $\begin{array}{l}\text { General; } \\
\text { cattle }\end{array}$ & Sreeramulu et al. 2013 \\
\hline Dalbergia melanoxylon Guill. and Perr. & Tree & Bark & Snakebite & General & Samy et al. 2008 \\
\hline Dalbergia pinnata (Lour.) Prain & Tree & Bark & Malarial treatment & General & Chander et al. 2015 \\
\hline Dalbergia saxatilis Hook. f. & Shrub & Leaves & Vegetable & General & Dansi et al. 2008 \\
\hline Dalbergia sissoo DC. & Tree & $\begin{array}{l}\text { Leaves, } \\
\text { bark }\end{array}$ & $\begin{array}{l}\text { Urine inflammation; diarrhoea; bleeding piles; skin } \\
\text { diseases; dysentery; diarrhoea; ulcer; leucoderma; } \\
\text { analgesic; antipyretic }\end{array}$ & General & Tetali et al. 2009 \\
\hline Dalbergia volubilis Roxb. & Shrub & $\begin{array}{l}\text { Bark, root, } \\
\text { leaves }\end{array}$ & Heart pain; menorrhagia; post-partum problem & $\begin{array}{l}\text { General; } \\
\text { women }\end{array}$ & Rao et al. 2011 \\
\hline Daniellia oliveri (Rolfe) Hutch. and Dalziel & Tree & Leaves & Vegetable & General & Dansi et al. 2008 \\
\hline Delonix elata (L.) Gamble & Tree & Bark & Fever & General & Padal et al. 2010 \\
\hline Delonix regia (Hook.) Raf. & Tree & $\begin{array}{l}\text { Flowers, } \\
\text { seed }\end{array}$ & Dysmenorrhoea; edible & General & Padal et al. 2014 \\
\hline Dendrolobium triangulare (Retz.) Schindl. & Shrub & Leaves & Prevents falling of hair & General & Ayyanar and Ignacimuthu 2005 \\
\hline Derris indica (Lam.) Bennet & Tree & Bark & Peripheral Neuritis & General & Padal et al. 2014 \\
\hline Derris scandens (Roxb.) Benth. & Tree & Root, bark & Tumours; snakebite and as fish poison; impaction & General & Dey et al. 2012, Raju 2008 \\
\hline Desmodium biflorum DC. & Herb & Whole plant & Jaundice & General & Asha and Pushpangadan 2002 \\
\hline Desmodium elegans DC. & Herb & Root, leaves & Cough; asthma & General & Kayani et al. 2014 \\
\hline Desmodium gangeticum (L.) DC. & Herb & Whole plant & $\begin{array}{l}\text { Anti-amnesic; immunomodulator; anti-diabetic; antioxidant; } \\
\text { cardioprotective; hepatoprotective; anti-inflammatory; } \\
\text { premature ejaculation; ulcer; eczema; gynecological } \\
\text { disorders; to cure piles; boils; blisters; scabies; ringworm; } \\
\text { toothache; headache; sedative; antipyretic; } \\
\text { antinociceptive; asthma; antidote of snake venom; health } \\
\text { tonic; typhoid; causes sterility in women; rheumatism; } \\
\text { diarrhoea; dysentery; piles }\end{array}$ & $\begin{array}{l}\text { General; } \\
\text { men; } \\
\text { women }\end{array}$ & $\begin{array}{l}\text { Bhattacharjee et al. 2013, } \\
\text { Chopda and Mahajan 2009, } \\
\text { Kosalge and Fursule 2009, } \\
\text { Reddy et al. 2008, Singh et al. } \\
2015\end{array}$ \\
\hline
\end{tabular}

Published 11 February 2019

http://dx.doi.org/10.32859/era.18.4.1-112 


\begin{tabular}{|c|c|c|c|c|c|}
\hline Desmodium laxiflorum DC. & Herb & Whole plant & $\begin{array}{l}\text { Stomach pain and fever; menstrual cycle irregularity; } \\
\text { uterus infection; jaundice }\end{array}$ & $\begin{array}{l}\text { General; } \\
\text { women }\end{array}$ & Singh et al. 2015 \\
\hline Desmodium oojeinense (Roxb.) H. Ohashi & Herb & Gum, bark & $\begin{array}{l}\text { Dysentry; diaherroea; to increase fertility and gain } \\
\text { pregnancy }\end{array}$ & $\begin{array}{l}\text { General; } \\
\text { women }\end{array}$ & Jain et al. 2004 \\
\hline Desmodium triflorum (L.) DC. & Herb & Whole plant & $\begin{array}{l}\text { Bone fracture; malaria; stomachache; piles; laxative; } \\
\text { bechic; epilepsy; dysentery; wound and abscesses; } \\
\text { antidiarrhoeal; galactogenic; sores; liver complaints; } \\
\text { dysentery; diarrhoea; increases lactation; carminative; } \\
\text { tonic; diuretic; gynaecological disorders }\end{array}$ & $\begin{array}{l}\text { General; } \\
\text { women }\end{array}$ & $\begin{array}{l}\text { Das et al. 2013, Lingaraju et al. } \\
2013 \text {, Shanmugam et al. 2012, } \\
\text { Singh et al. } 2015\end{array}$ \\
\hline Dialium guineense Willd. & Herb & Leaves & Vegetable & General & Dansi et al. 2008 \\
\hline Dicerma biarticulatum (L.) DC. & Herb & Leaves & Boils & General & Padal et al. 2014 \\
\hline Dichrostachys cinerea (L.) Wight and Arn. & Tree & $\begin{array}{l}\text { Root, } \\
\text { leaves, } \\
\text { flower, bark }\end{array}$ & $\begin{array}{l}\text { Boils; blisters; ulcer; indigestion; allergy and pains due to } \\
\text { wounds; snakebite; rheumatism; toothache; chicken pox; } \\
\text { hair tonic; reduces menstural flow; vomiting; }\end{array}$ & $\begin{array}{l}\text { General; } \\
\text { women }\end{array}$ & $\begin{array}{l}\text { Padal et al. 2010, Rao et al. } \\
\text { 2006, Tetali et al. } 2009\end{array}$ \\
\hline Dolichos aciphyllus R. Wilczek & $\begin{array}{l}\text { Herb or } \\
\text { shrub }\end{array}$ & Whole plant & Swelling & General & Nizar et al. 2015 \\
\hline Dolichos trilobus L. & $\begin{array}{l}\text { Herb or } \\
\text { shrub }\end{array}$ & Whole plant & Abortifacient & $\begin{array}{l}\text { Pregnant } \\
\text { women }\end{array}$ & Krishnaraj et al. 2012 \\
\hline Entada africana Guill. and Perr. & Tree & Bark & Symptoms of chest pain & General & Nanjunda 2010 \\
\hline Entada phaseoloides (L.) Merr. & Tree & Endosperm & Rheumatism & General & Sudhadevi 1999 \\
\hline Entada rheedii Spreng. & Tree & $\begin{array}{l}\text { Seed, bark, } \\
\text { leaves, root, } \\
\text { endosperm }\end{array}$ & $\begin{array}{l}\text { Rheumatism; stomachache; fever; pain; cold; scabies; } \\
\text { contraceptive; intestinal worms; skin disease; infantile } \\
\text { cold; epilepsy; snake bite; hypertension; dysentery }\end{array}$ & $\begin{array}{l}\text { General; } \\
\text { women; } \\
\text { children }\end{array}$ & $\begin{array}{l}\text { Padal et al. 2014, Sai Vishnu } \\
\text { Priya and Rao } 2008\end{array}$ \\
\hline Erythrina excelsa Baker & Tree & Bark & Snakebite & General & Samy et al. 2008 \\
\hline Erythrina suberosa Roxb. & Tree & Seed, root & Leucorrhoea; dysentery; cattle wounds & $\begin{array}{l}\text { General; } \\
\text { cattle }\end{array}$ & $\begin{array}{l}\text { Kshirsagar and Singh 2001, } \\
\text { Sreeramulu et al. } 2013\end{array}$ \\
\hline Erythrina variegata $\mathrm{L}$. & Tree & Aerial parts & $\begin{array}{l}\text { Snake bite; gall bladder stones; vermifuge; anthelmintic; } \\
\text { aphrodisiac; laxative; diuretic; galactagogue; rheumatic } \\
\text { joints; fever; malaria; burns; regulates menstrual flow; } \\
\text { paralysis; asthma; eczema; ear problems; leprosy; } \\
\text { epilepsy; gynaecological disorders; aching of mammary } \\
\text { glands; impaction; cold; cough }\end{array}$ & $\begin{array}{l}\text { General; } \\
\text { women }\end{array}$ & Pushpangadan and Atal 1984 \\
\hline Flemingia macrophylla (Willd.) Merr. & Shrub & Root & - & General & Sharma et al. 2013 \\
\hline Flemingia procumbens Roxb. & Shrub & Root & - & General & Sharma et al. 2013 \\
\hline Flemingia stricta Roxb. & Shrub & Aerial parts & Obstetric diseases; body pain; cold & General & Srithi et al. 2009 \\
\hline Flemingia strobilifera (L.) W.T. Aiton & Shrub & $\begin{array}{l}\text { Root, } \\
\text { leaves, } \\
\text { flower }\end{array}$ & Malaria; body pain; swelling; epilepsy & General & $\begin{array}{l}\text { Reddy et al. 2008, Sharma et al. } \\
2013\end{array}$ \\
\hline Galactia longifolia (Jacq.) Benth. & Herb & Leaves & Cuts & General & Rajendran et al. 2000 \\
\hline Galactia tenuiflora (Willd.) Wight and Arn. & Herb & Legume & Edible & General & Bhagat et al. 2016 \\
\hline Gliricidia sepium (Jacq.) Walp. & Tree & Root & Snake bite & General & Chander et al. 2015 \\
\hline Glycine max (L.) Merr. & Herb & Seed & Snakebite & General & Samy et al. 2008 \\
\hline Glycyrrhiza glabra L. & $\begin{array}{l}\text { Perennial } \\
\text { shrub }\end{array}$ & Whole plant & Cough; bronchitis; constipation; asthma; cold; throat pain & General & $\begin{array}{l}\text { Chopda and Mahajan 2009, } \\
\text { Sharma et al. } 2013\end{array}$ \\
\hline
\end{tabular}

Published 11 February 2019

http://dx.doi.org/10.32859/era.18.4.1-112 


\begin{tabular}{|c|c|c|c|c|c|}
\hline Gymnocladus assamicus P.C. Kanjilal & Tree & Pods & Detergent (soap); religious and veterinary & $\begin{array}{l}\text { General; } \\
\text { veterinary }\end{array}$ & Ruba and Mohan 2016 \\
\hline Humboldtia decurrens Oliv. & Tree & Root & Anti-snake venom & General & Sulochana et al. 2015 \\
\hline Indigofera aspalathoides DC. & Herb & Whole plant & Skin diseases; toothache & General & Kumar et al. 2007 \\
\hline Indigofera cassioides DC. & Herb & Flower & Stomachache & General & Kshirsagar and Singh 2001 \\
\hline Indigofera linifolia (L.f.) Retz. & Herb & Whole plant & Skin diseases & General & $\begin{array}{l}\text { Prashantkumar and Vidyasagar } \\
2008\end{array}$ \\
\hline Indigofera linnaei Ali & Herb & Whole plant & Antifertility & General & Sharma et al. 2013 \\
\hline Indigofera mysorensis DC. & Herb & Leaves & Cuts and skin diseases & General & Rao et al. 2006 \\
\hline Indigofera nummulariifolia (L.) Alston & Herb & Whole plant & Liver complaints & General & Jayanthi et al. 2012 \\
\hline Indigofera tinctoria L. & Herb & Whole plant & $\begin{array}{l}\text { Liver diseases; snake bites; allopatia; bronchitis; cough; } \\
\text { respiratory infections; tuberculosis; jaundice }\end{array}$ & General & $\begin{array}{l}\text { Dey and De 2012, Padal et al. } \\
2010\end{array}$ \\
\hline Indigofera trita L.f. & Herb & $\begin{array}{l}\text { Seed, } \\
\text { leaves }\end{array}$ & Impaction; nutritive tonic & General & Sreeramulu et al. 2013 \\
\hline Isoberlinia doka Craib and Stapf & Tree & Leaves & Vegetable & General & Dansi et al. 2008 \\
\hline Kingiodendron pinnatum (DC.) Harms & Tree & Gum & Gonorrhea & General & Prasad et al. 2014 \\
\hline $\begin{array}{l}\text { Kunstleria keralensis C.N. Mohanan and } \\
\text { N.C. Nair }\end{array}$ & $\begin{array}{l}\text { Climber or } \\
\text { liana }\end{array}$ & Bark & Body pain & General & Binu 2011 \\
\hline Lablab purpureus (L.) Sweet & Forb/herb & Leaves & Ring worm & General & Umapriya et al. 2011 \\
\hline Leucaena leucocephala (Lam.) de Wit & Tree & Legume & Edible & General & Bhagat et al. 2016 \\
\hline Macrotyloma uniflorum (Lam.) Verdc. & Beans & Seed & Abortifacient & $\begin{array}{l}\text { Pregnant } \\
\text { women }\end{array}$ & Rajan et al. 1997 \\
\hline Milletia elliptica (Roxb.) Steud. & Tree & $\begin{array}{l}\text { Flower, } \\
\text { leaves, bark }\end{array}$ & Diabetes; bone fracture; fish poison & General & SM and Vidyasagar 2013 \\
\hline Mimosa hamata Willd. & Shrub & $\begin{array}{l}\text { Flower, root, } \\
\text { bark }\end{array}$ & $\begin{array}{l}\text { Fertility; leucorrhoea and menorrhagia; stomach ache after } \\
\text { delivery }\end{array}$ & $\begin{array}{l}\text { General; } \\
\text { women }\end{array}$ & $\begin{array}{l}\text { Jain et al. 2004, Sreeramulu et } \\
\text { al. } 2013\end{array}$ \\
\hline Mimosa pudica L. & Shrub & Whole plant & $\begin{array}{l}\text { Asthma; blood purifier; diarrhoea; dysentery; snake bite; } \\
\text { scorpion sting; wound; body pain; jaundice; cuts; piles; } \\
\text { stomachache; muscular pains; psoriasis; stops bleeding; } \\
\text { abortifacient; blood in breast milk; insomnia; fever; } \\
\text { dysmenorrhea; urinary disorder; skin problems; fistula; for } \\
\text { inducing sleep in children }\end{array}$ & $\begin{array}{l}\text { General; } \\
\text { children; } \\
\text { women }\end{array}$ & $\begin{array}{l}\text { Pushpangadan and Atal 1984, } \\
\text { Samy et al. 2008, Sharma et al. } \\
2013 \text {, Udayan et al. 2005, } \\
\text { Udayan et al. } 2007\end{array}$ \\
\hline Mucuna gigantea (Willd.) DC. & $\begin{array}{l}\text { Climbing } \\
\text { liana }\end{array}$ & Seed & Sexual problems & General & Prasad et al. 2014 \\
\hline Mucuna nigricans (Lour.) Steud. & $\begin{array}{l}\text { Climbing } \\
\text { liana }\end{array}$ & Seed & Genital ulcer & General & Nanjunda 2010 \\
\hline Mucuna pruriens (L.) DC. & $\begin{array}{l}\text { Climbing } \\
\text { liana }\end{array}$ & Whole plant & $\begin{array}{l}\text { Diarrhea; snakebite; sexual disorder; amenorrhea; } \\
\text { abnormal mensturation; boils; blisters; wound; } \\
\text { anthelmintic; allergy; rheumatoid arthritis; earache; } \\
\text { intestinal worms; gastrointestinal disorders; seminal } \\
\text { weakness; kidney stones }\end{array}$ & $\begin{array}{l}\text { General; } \\
\text { men }\end{array}$ & $\begin{array}{l}\text { Bhaskar and Samant 2012, } \\
\text { Gayakvad et al. } 2014\end{array}$ \\
\hline Mundulea sericea (Willd.) A. Chev. & $\begin{array}{l}\text { Climbing } \\
\text { liana }\end{array}$ & Leaves & Whooping cough & General & Rao et al. 2006 \\
\hline Ormocarpum sennoides (Willd.) DC. & Shrub & Leaves & Vegetable & General & Dansi et al. 2008 \\
\hline
\end{tabular}

Published 11 February 2019

http://dx.doi.org/10.32859/era.18.4.1-112 


\begin{tabular}{|c|c|c|c|c|c|}
\hline Peltophorum pterocarpum (DC.) K. Heyne & Tree & Bark, flower & $\begin{array}{l}\text { Wound; dysentery; eye diseases; muscular pains; sores; } \\
\text { antibacterial; insomnia; induces sleep; toothache; pain }\end{array}$ & General & Duraipandiyan et al. 2006 \\
\hline Phaseolus vulgaris $\mathrm{L}$. & Herb/Bean & Leaves & Vegetable & General & Dansi et al. 2008 \\
\hline Phyllodium pulchellum (L.) Desv. & Shrub & Leaves & Menorrhagia & Women & Lingaraju et al. 2013 \\
\hline Piliostigma malabaricum (Roxb.) Benth. & Tree & Whole plant & Fodder & Animals & Gavali and Sharma 2004 \\
\hline Pisum sativum $\mathrm{L}$. & Herb & Leaves & - & General & Chopda and Mahajan 2009 \\
\hline Pithecellobium dulce (Roxb.) Benth. & Tree & Whole plant & Dysentery; intestinal disorders; edible & \begin{tabular}{|l|} 
General \\
\end{tabular} & Bhagat et al. 2016 \\
\hline Pongamia pinnata (L.) Pierre & Tree & $\begin{array}{l}\text { Bark, wood, } \\
\text { shoot, } \\
\text { flower, } \\
\text { leaves, oil, } \\
\text { twig, root, } \\
\text { seed }\end{array}$ & $\begin{array}{l}\text { Skin diseases; piles; worm infection; rheumatism; asthma; } \\
\text { antidiarrhoeal; malaria; scabies; herpes; stomach ulcers; } \\
\text { diabetes; headache; ringworm; elephant scratches; } \\
\text { snakebite; centipede bite; headache; itching }\end{array}$ & General & $\begin{array}{l}\text { Rao et al. 2006, Umapriya et al. } \\
2011\end{array}$ \\
\hline Prosopis cineraria (L.) Druce & Tree & $\begin{array}{l}\text { Bark, flower, } \\
\text { leaves, gum }\end{array}$ & $\begin{array}{l}\text { Snakebite; to develop complete sterility; abortion; } \\
\text { toothache; cold; cough; fever; prevents miscarriage; boils; } \\
\text { skin diseases; rheumatism; dysentery; cataract; } \\
\text { dyspepsia; earache; fever }\end{array}$ & $\begin{array}{l}\text { General; } \\
\text { women; } \\
\text { pregnant } \\
\text { women } \\
\end{array}$ & Jain et al. 2004 \\
\hline Prosopis juliflora (Sw.) DC. & Tree & $\begin{array}{l}\text { Bark, } \\
\text { leaves, pod, } \\
\text { flower, fruit }\end{array}$ & $\begin{array}{l}\text { Oral health care; dyspepsia; earache; mouth infections; } \\
\text { scorpion stings; snake bites; asthma; edible; joint pain }\end{array}$ & General & $\begin{array}{l}\text { Bhagat et al. 2016, Kayani et al. } \\
2014\end{array}$ \\
\hline Pseudarthria viscida (L.) Wight and Arn. & Shrub & Whole plant & $\begin{array}{l}\text { Fever; asthma; diabetes; helminthiasis; piles; strangury; } \\
\text { asthma; cardiovascular diseases; nerve disorder; snake } \\
\text { bite; ulcer; bronchitis; hepatic disorder; cold; cough; } \\
\text { postpartum health; prolapse of uterus }\end{array}$ & $\begin{array}{l}\text { General; } \\
\text { women }\end{array}$ & $\begin{array}{l}\text { Ayyanar and Ignacimuthu 2005, } \\
\text { Padal et al. } 2014\end{array}$ \\
\hline Psophocarpus palustris Desv. & Shrub & Leaves & Vegetable & General & Dansi et al. 2008 \\
\hline Pterocarpus marsupium Roxb. & Tree & Whole plant & $\begin{array}{l}\text { Diabetes; labour pains; fertility; jaundice; menorrhegia; } \\
\text { stomachache; menstural problems; body pain; toothache; } \\
\text { mouth ulcers; skin diseases; fever }\end{array}$ & General & $\begin{array}{l}\text { Chopda and Mahajan 2009, Jain } \\
2004\end{array}$ \\
\hline Pterocarpus santalinoides DC. & Tree & Leaves & Vegetable & General & Dansi et al. 2008 \\
\hline Pterocarpus santalinus L.f. & Tree & $\begin{array}{l}\text { Bark, } \\
\text { leaves, } \\
\text { wood, bark }\end{array}$ & $\begin{array}{l}\text { Rheumatism; blood purifier; diuretic; boils; blisters; } \\
\text { snakebite }\end{array}$ & General & $\begin{array}{l}\text { Chopda and Mahajan 2009, } \\
\text { Padma et al. } 2016\end{array}$ \\
\hline $\begin{array}{l}\text { Pterolobium hexapetalum (Roth) } \\
\text { Santapau and Wagh }\end{array}$ & Shrub & $\begin{array}{l}\text { Leaves, } \\
\text { bark, wood }\end{array}$ & $\begin{array}{l}\text { Reduces delivery pain; fever; tooth ache; chest pain; } \\
\text { delivery pains; antidote to dog bite; wound healing; } \\
\text { constipation; piles; ulcers; skin infections; venereal } \\
\text { diseases; jaundice; diarrhoea; fire wood }\end{array}$ & General & Duraipandiyan et al. 2006 \\
\hline Pueraria tuberosa (Willd.) DC. & Climber & $\begin{array}{l}\text { Leaves, } \\
\text { tuber, bulb, } \\
\text { root }\end{array}$ & $\begin{array}{l}\text { Joint disease; insecticidal; wormicidal; rheumatism; fever; } \\
\text { headache; heart pain; increases lactation; amenorrhegia; } \\
\text { impotency }\end{array}$ & $\begin{array}{l}\text { General; } \\
\text { women }\end{array}$ & $\begin{array}{l}\text { Bhardwaj et al. 2011, Padal et } \\
\text { al. 2010, Sayed et al. 2007, } \\
\text { Sreeramulu et al. } 2013\end{array}$ \\
\hline Rhynchosia beddomei Baker & $\begin{array}{l}\text { Perennial } \\
\text { non-climbing } \\
\text { shrub }\end{array}$ & Leaves & Skin disease & General & Ram et al. 2004 \\
\hline Rhynchosia bracteata Baker & $\begin{array}{l}\text { Perennial } \\
\text { non-climbing } \\
\text { shrub }\end{array}$ & Root & Dysentery & General & Kosalge and Fursule 2009 \\
\hline
\end{tabular}

Published 11 February 2019

http://dx.doi.org/10.32859/era.18.4.1-112 
Ethnobotany Research and Applications

\begin{tabular}{|c|c|c|c|c|c|}
\hline Rhynchosia cana (Willd.) DC. & $\begin{array}{l}\text { Perennial } \\
\text { non-climbing } \\
\text { shrub }\end{array}$ & Seed & Anti-inflammation and antipyretic & General & Kanthasamy Kalaichelvi 2016 \\
\hline Rhynchosia densiflora (Roth) DC. & $\begin{array}{l}\text { Perennial } \\
\text { non-climbing } \\
\text { shrub }\end{array}$ & Root & Dysentery & General & Sarvalingam et al. 2011 \\
\hline Rhynchosia rufescens (Willd.) DC. & $\begin{array}{l}\text { Perennial } \\
\text { non-climbing } \\
\text { shrub }\end{array}$ & Leaves & Abortiacient & $\begin{array}{l}\text { Pregnant } \\
\text { women }\end{array}$ & Krishnaraj et al. 2012 \\
\hline Saraca asoca (Roxb.) Willd. & Tree & $\begin{array}{l}\text { Bark, flower, } \\
\text { seed, stem }\end{array}$ & $\begin{array}{l}\text { Abortifacient; bed sore; menstural disorders; skin disease; } \\
\text { dysentery; urinary disorder }\end{array}$ & $\begin{array}{l}\text { General; } \\
\text { women; } \\
\text { pregnant } \\
\text { women }\end{array}$ & $\begin{array}{l}\text { Bhat et al. 2014, Das et al. } \\
\text { 2014, Padal et al. } 2014\end{array}$ \\
\hline Saraca indica L. & Tree & Bark & Urinary problems & General & Chopda and Mahajan 2009 \\
\hline Senna alata (L.) Roxb. & Shrub & $\begin{array}{l}\text { Leaves, } \\
\text { bark, flower, } \\
\text { root }\end{array}$ & $\begin{array}{l}\text { Skin diseases; fractures; abortifacient; contraceptive; } \\
\text { scabies; eczema and leucoderma; Cattle alopecia }\end{array}$ & $\begin{array}{l}\text { General; } \\
\text { cattle }\end{array}$ & $\begin{array}{l}\text { Kshirsagar and Singh } 2001 \text {, } \\
\text { Samy et al. } 2008 \text {, Sripathi and } \\
\text { Sankari } 2010\end{array}$ \\
\hline Senna alexandrina Mill. & Herb & $\begin{array}{l}\text { Leaves, } \\
\text { bark }\end{array}$ & $\begin{array}{l}\text { Dysentery; piles; constipation; sour throat; head ache and } \\
\text { giddiness; laxative; diarrhoea }\end{array}$ & General & $\begin{array}{l}\text { Jaganathan et al. 2016, Rao et } \\
\text { al. } 2006\end{array}$ \\
\hline Senna auriculata (L.) Roxb. & Herb & Whole plant & $\begin{array}{l}\text { Dandruff; Stop drinking; body ache; excessive menstural } \\
\text { flow; diabetes; eye infections; constipation; liver disease; } \\
\text { urinary tract diseases; scorpion bite; ulcers; leprosy; bone } \\
\text { fractures; scabies; blood purifier; strengtening of heart; } \\
\text { anthelmintic; conjuctivitis }\end{array}$ & $\begin{array}{l}\text { Women; } \\
\text { General }\end{array}$ & Bosco and Arumugam 2012 \\
\hline $\begin{array}{l}\text { Senna candolleana (Vogel) H.S. Irwin and } \\
\text { Barneby }\end{array}$ & Herb & $\begin{array}{l}\text { Leaves, } \\
\text { stem, fruit }\end{array}$ & Cure various ailments; stomachache & General & Ignacimuthu et al. 2008 \\
\hline Senna hirsuta (L.) H.S. Irwin and Barneby & Shrub & Leaves, root & Snakebite; oral health care & General & Hebbar et al. 2004 \\
\hline Senna italica Mill. & Herb & $\begin{array}{l}\text { Leaves, } \\
\text { whole plant }\end{array}$ & Gastrointestinal disorders; constipation; diabetes & General & $\begin{array}{l}\text { SM et al. } 2013 \text {, Sreeramulu et } \\
\text { al. } 2013\end{array}$ \\
\hline Senna montana (Roth) V. Singh & Herb & Leaves & Rheumatic pains & General & Savithramma et al. 2014 \\
\hline $\begin{array}{l}\text { Senna multiglandulosa (Jacq.) H.S. Irwin } \\
\text { and Barneby }\end{array}$ & Herb & Leaves & Antifungal; antimicrobial; eczema and intestinal parasities & General & Kanthasamy Kalaichelvi 2016 \\
\hline $\begin{array}{l}\text { Senna obtusifolia (L.) H.S. Irwin and } \\
\text { Barneby }\end{array}$ & Herb & Leaves & Insect bite; vegetable & General & Sreeramulu et al. 2013 \\
\hline Senna occidentalis (L.) Link & Herb & Whole plant & $\begin{array}{l}\text { To treat malaria and malaria-like symptoms; skin troubles; } \\
\text { bone fractures; toothache; insect bite; swelling; antidote; } \\
\text { snakebite; tonsilitis; sore throat; blood tonic; rheumatoid } \\
\text { arthritis; cutaneous diseases }\end{array}$ & General & $\begin{array}{l}\text { Pradheeps and Poyyamoli } 2013 \\
\text { Rao et al. 2006, Ratnam and } \\
\text { Raju } 2008\end{array}$ \\
\hline Senna occidentalis (L.) Link & Herb & Leaves & Rheumatism; vegetable & General & Dansi et al. 2008 \\
\hline $\begin{array}{l}\text { Senna siamea (Lam.) H.S. Irwin and } \\
\text { Barneby }\end{array}$ & Herb & - & Fire wood & General & Prabakaran et al. 2013 \\
\hline Senna sophera (L.) Roxb. & Herb & Whole plant & $\begin{array}{l}\text { Diabetes; bronchitis; ulcers; Bronchial asthma; respiratory } \\
\text { dyspnoea; fever; cold; cough; influenza; malaria }\end{array}$ & General & $\begin{array}{l}\text { Jain 2004, Kanthasamy } \\
\text { Kalaichelvi 2016, Padal et al. } \\
2010\end{array}$ \\
\hline
\end{tabular}

Published 11 February 2019

http://dx.doi.org/10.32859/era.18.4.1-112 


\begin{tabular}{|c|c|c|c|c|c|}
\hline Senna tora (L.) Roxb. & Herb & Whole plant & $\begin{array}{l}\text { Typhoid; leprosy and ulcers; skin diseases; eczema; } \\
\text { snake bite; diarrhoea; antibacterial; insecticidal; } \\
\text { wormicidal; indigestion; ringworm; laxative }\end{array}$ & General & $\begin{array}{l}\text { Bhat et al. 2014, Hebbar et al. } \\
2004 \text {, Pushpangadan and Atal } \\
1984\end{array}$ \\
\hline Sesbania bispinosa (Jacq.) W. Wight & Tree & Flower & Nose bleeding & General & Patil and Ahirrao 2011 \\
\hline Sesbania grandiflora (L.) Pers. & Tree & $\begin{array}{l}\text { Bark, flower, } \\
\text { leaves }\end{array}$ & $\begin{array}{l}\text { Antibacterial; malaria; headache; improves eye vision; } \\
\text { eliminates worms; dysentery; stomachache; mouth ulcer; } \\
\text { canker sores }\end{array}$ & General & $\begin{array}{l}\text { Padal et al. 2014, Valsaraj et al } \\
1997\end{array}$ \\
\hline Sesbania javanica Miq. & Tree & Leaves & Toothache & General & Ignacimuthu et al. 2008 \\
\hline Sesbania sesban (L.) Merr. & Tree & $\begin{array}{l}\text { Seed, } \\
\text { leaves }\end{array}$ & Dysentry; diaherroea; fever & General & Gairola et al. 2013 \\
\hline Shuteria involucrata (Wall.) Wight and Arn. & Herb & Leaves & Boils & General & Deepak and Gopal 2014 \\
\hline Smithia conferta Sm. & Herb & Whole plant & $\begin{array}{l}\text { To make women fertile and become pregnant; preserves } \\
\text { youth; removes wrinkles and makes the body tender; root } \\
\text { for impotency and seminal weakness in men. }\end{array}$ & $\begin{array}{l}\text { General; } \\
\text { women; } \\
\text { men }\end{array}$ & Pushpangadan and Atal 1984 \\
\hline Smithia sensitiva Aiton & Herb & Whole plant & Antibacterial & General & Ranjitha et al. 2016 \\
\hline Sophora flavescens Aiton & Herb & - & Anti-inflammatory & General & Zhang et al. 2013 \\
\hline Spatholobus suberectus Dunn & Herb & - & Anti-bacterial & General & Zhang et al. 2013 \\
\hline Stylosanthes fruticosa (Retz.) Alston & Herb & Whole plant & Hip pain & General & Ignacimuthu et al. 2008 \\
\hline Styphnolobium japonicum (L.) Schott & Herb & - & Anti-tumour & General & Zhang et al. 2013 \\
\hline Tamarindus indica $\mathrm{L}$. & Tree & Whole plant & $\begin{array}{l}\text { Abortifacient; piles; bone fracture; burns; eye infection; } \\
\text { herpes; skin disease; snakebite; scorpion sting; malaria; } \\
\text { oral ulcers; sore mouth; indigestion; laxative }\end{array}$ & $\begin{array}{l}\text { General; } \\
\text { pregnant } \\
\text { women }\end{array}$ & $\begin{array}{l}\text { Bhagat et al. 2016, Chander et } \\
\text { al. 2015, Pradheeps and } \\
\text { Poyyamoli } 2013\end{array}$ \\
\hline Tephrosia purpurea (L.) Pers. & $\begin{array}{l}\text { Perennial } \\
\text { erect herb }\end{array}$ & Whole plant & $\begin{array}{l}\text { Diabetes; asthma; snakebite; tumors; ulcers; liver } \\
\text { diseases; fever; kidney diseases; scorpion sting; cough; } \\
\text { stomach pain; urinary disorders; comiting; post natal } \\
\text { complication of women; skin diseases }\end{array}$ & $\begin{array}{l}\text { General; } \\
\text { women }\end{array}$ & $\begin{array}{l}\text { Chopda and Mahajan 2009, } \\
\text { Dhal et al. 2011, Jain } 2004\end{array}$ \\
\hline Tephrosia villosa (L.) Pers. & $\begin{array}{l}\text { Perennial } \\
\text { erect herb }\end{array}$ & Root, leaves & Typhoid; skin disease & General & Porte 2014 \\
\hline Teramnus labialis (L.f.) Spreng. & Herb & Legume & Edible & General & Bhagat et al. 2016 \\
\hline $\begin{array}{l}\text { Trichopodium zeylanicum (Gaertn.) } \\
\text { Thwaites }\end{array}$ & Herb & Whole plant & $\begin{array}{l}\text { Immunomodulatory; antibacterial; hepatoprotective; anti- } \\
\text { ulcer; aphrodisiac; veneral diseases; improves body vigour }\end{array}$ & General & $\begin{array}{l}\text { Ayyanar and Ignacimuthu } 2005 \\
\text { Udayan et al. } 2007\end{array}$ \\
\hline Trifolium pratense $\mathrm{L}$. & Herb & Flower & Coughs; sore throat & General & Kayani et al. 2014 \\
\hline Trigonella foenum-graecum $\mathrm{L}$. & Herb & Seed & Diabetes; increases lactation; body cooling & $\begin{array}{l}\text { General; } \\
\text { women }\end{array}$ & Ignacimuthu et al. 2008 \\
\hline Uraria lagopodoides (L.) DC. & Herb & Whole plant & Abortion & $\begin{array}{l}\text { Pregnant } \\
\text { women }\end{array}$ & Murty and Venkaiah 2010 \\
\hline Uraria picta (Jacq.) DC. & Herb & $\begin{array}{l}\text { Flower, } \\
\text { leaves, seed }\end{array}$ & Respiratory disorder; expells tapeworm & General & Wagh and Jain 2015 \\
\hline Vigna aconitifolia (Jacq.) Marechal & $\begin{array}{l}\text { Erect } \\
\text { herb/Beans }\end{array}$ & Fruit & Skin diseases & General & Savithramma et al. 2014 \\
\hline $\begin{array}{l}\text { Vigna khandalensis (Santapau) } \\
\text { Sundararagh. and Wadhw }\end{array}$ & Erect herb & Seed & Edible & General & Bhagat et al. 2016 \\
\hline Vigna radiata (L.) R. Wilczek & Erect herb & Root, fruit & Boils; digestion; constipation & General & Bhat et al. 2014 \\
\hline Vigna trilobata (L.) Verdc. & Erect herb & Whole plant & Fever & General & Chopda and Mahajan 2009 \\
\hline Vigna unguiculata (L.) Walp. & Erect herb & Leaves & Vegetable & General & Dansi et al. 2008 \\
\hline
\end{tabular}

Published 11 February 2019

http://dx.doi.org/10.32859/era.18.4.1-112 


\begin{tabular}{|c|c|c|c|c|c|}
\hline Vigna vexillata (L.) A. Rich. & Erect herb & Seed & Edible & General & Bhagat et al. 2016 \\
\hline Xylia xylocarpa (Roxb.) Taub. & Tree & Seed, bark & Diarrhoea; edible; leprosy; rheumatoid arthritis & General & Mahishi et al. 2005 \\
\hline Zornia diphylla (L.) Pers. & Herb & Whole plant & Stomach ulcer & General & Ayyanar and Ignacimuthu 2005 \\
\hline \multicolumn{6}{|l|}{ Fagaceae } \\
\hline Castanopsis indica (Roxb. ex Lindl.) A.DC. & Tree & $\begin{array}{l}\text { Leaves, } \\
\text { stem bark }\end{array}$ & Fish poison and raw seeds are eaten & General & Ruba and Mohan 2016 \\
\hline Quercus oblongata D. Don & $\begin{array}{l}\text { Shrub to } \\
\text { small tree }\end{array}$ & Gum, bark & Asthma; gonorrheal; digestive disorders & General & Kumar et al. 2011 \\
\hline Quercus robur $\mathrm{L}$. & Tree & Seed, fruit & Asthma & General & Kayani et al. 2014 \\
\hline \multicolumn{6}{|l|}{ Fissidentaceae } \\
\hline Fissidens nobilis Griff. & Epiphyte & - & Diuretic; hair growth & General & Chandra et al. 2016 \\
\hline \multicolumn{6}{|l|}{ Frullaniaceae } \\
\hline Frullania ericoides (Nees ex Mart.) Mont. & Liverwort & Whole plant & Nourishment of hair & General & Chandra et al. 2016 \\
\hline \multicolumn{6}{|l|}{ Gentianaceae } \\
\hline Canscora alata (Roth) Wall. & Herb & Leaves & Nervine tonic & General & Wagh and Jain 2015 \\
\hline $\begin{array}{l}\text { Canscora diffusa (Vahl) R. Br. ex Roem. } \\
\text { and Schult. }\end{array}$ & Herb & Whole plant & Malarial treatment & General & Jain 2004 \\
\hline $\begin{array}{l}\text { Enicostema axillare (Poir. ex Lam.) A. } \\
\text { Raynal }\end{array}$ & Herb & Whole plant & $\begin{array}{l}\text { Loose motions; vomiting; fever; malaria; blood purification; } \\
\text { diabetes; insect poisoning; diuretic; snake and scorpion } \\
\text { bite; boils; blisters; wound; antibacterial }\end{array}$ & General & $\begin{array}{l}\text { Padal et al. 2010, Sharma and } \\
\text { Mujundar 2003, Wagh and Jain } \\
2015\end{array}$ \\
\hline Exacum tetragonum Roxb. & Herb & Whole plant & Asthma; sores of leech bite & General & Lingaraju et al. 2013 \\
\hline Gentiana olivieri Griseb. & Herb & Whole plant & Cough; chest problems; throat problems & General & Kayani et al. 2014 \\
\hline $\begin{array}{l}\text { Swertia angustifolia Buch. -Ham. ex D. } \\
\text { Don }\end{array}$ & Herb & Whole plant & Fever; indigestion; diarrhea; scabies & General & Acharya and Kaphle 2015 \\
\hline Swertia chirata Buch. -Ham. ex Wall. & Tree & $\begin{array}{l}\text { Leaves, } \\
\text { stem }\end{array}$ & Fever & General & Chopda and Mahajan 2009 \\
\hline $\begin{array}{l}\text { Swertia chirayita (Roxb.) Buch. -Ham. ex } \\
\text { C.B. Clarke }\end{array}$ & Herb & Whole plant & $\begin{array}{l}\text { Malaria; liver disorders; nausea; fevers; indigestion; } \\
\text { scorpion bite; skin diseases; gastrointestinal infections; } \\
\text { diabetes }\end{array}$ & General & $\begin{array}{l}\text { Dhal et al. 2011, Eneh et al. } \\
2013\end{array}$ \\
\hline Swertia lawii Burkill & Herb & Whole plant & Antibacterial & General & Ranjitha et al. 2016 \\
\hline Swertia multicaulis D. Don & Tree & Whole plant & Cuts and wounds & General & Acharya and Kaphle 2015 \\
\hline Swertia petiolata D. Don & Herb & Whole plant & Pneumonia fever; throat problems; bronchitis; asthma & General & Kayani et al. 2014 \\
\hline $\begin{array}{l}\text { Swertia racemosa (Wall. ex Griseb.) C.B. } \\
\text { Clarke }\end{array}$ & Herb & Whole plant & Fever; malaria; jaundice; diabetes; cold; cough; headache & General & Acharya and Kaphle 2015 \\
\hline \multicolumn{6}{|l|}{ Geraniaceae } \\
\hline Geranium mascatense Boiss. & Herb & Whole plant & Liver troubles and fever & General & Kumar et al. 2011 \\
\hline \multicolumn{6}{|l|}{ Gesneriaceae } \\
\hline Henckelia incana (Vahl) Spreng. & Herb & Leaves & Ear pain & General & Hills 2010 \\
\hline \multicolumn{6}{|l|}{ Ginkgoaceae } \\
\hline Ginkgo biloba L. & Tree & - & - & - & Ross 2003 \\
\hline \multicolumn{6}{|l|}{ Gisekiaceae } \\
\hline Gisekia pharnaceoides L. & Herb & Whole plant & Insecticidal; wormicidal & General & Bhardwaj et al. 2011 \\
\hline
\end{tabular}




\begin{tabular}{|c|c|c|c|c|c|}
\hline Dicranopteris linearis (Burm. f.) Underw. & Herb & Leaves & $\begin{array}{l}\text { To reduce body temperature and to control fever; to treat } \\
\text { external wounds; ulcers; and boils; to treat asthma and } \\
\text { women's sterility }\end{array}$ & $\begin{array}{l}\text { General; } \\
\text { women }\end{array}$ & Zakaria et al. 2008 \\
\hline \multicolumn{6}{|l|}{ Hernandiaceae } \\
\hline Gyrocarpus americanus Jacq. & Tree & Bark & Spider bite & General & Ratnam and Raju 2008 \\
\hline \multicolumn{6}{|l|}{ Hydrocharitaceae } \\
\hline Ottelia alismoides (L.) Pers. & $\begin{array}{l}\text { Aquatic } \\
\text { submerged } \\
\text { herb }\end{array}$ & Leaves & Fractures; snake bite & General & Rao et al. 2006 \\
\hline Vallisneria natans (Lour.) H. Hara & Herb & Bark & Skin diseases; cuts & General & Rao et al. 2006 \\
\hline \multicolumn{6}{|l|}{ Hydroleaceae } \\
\hline Hydrolea glabra Schumach. and Thonn. & Herb & Leaves & Vegetable & General & Dansi et al. 2008 \\
\hline Hydrolea zeylanica (L.) Vahl & Herb & Leaves, root & Wound healing; ulcers & General & Kanthasamy Kalaichelvi 2016 \\
\hline \multicolumn{6}{|l|}{ Hypericaceae } \\
\hline Hypericum gaitii Haines & Shrub & Leaves & Skin diseases like eczema and herpes & General & $\begin{array}{l}\text { Muralidhara Rao and Pullaiah } \\
2007\end{array}$ \\
\hline Hypericum perforatum L. & Herb & Root & - & \begin{tabular}{|l|} 
General \\
\end{tabular} & Kataria and Kaur 2013 \\
\hline \multicolumn{6}{|l|}{ Hypoxidaceae } \\
\hline Curculigo orchioides Gaertn. & $\begin{array}{l}\text { Rhizomatic } \\
\text { herb }\end{array}$ & Whole plant & $\begin{array}{l}\text { Skin diseases; piles; blood related disorders; bronchitis; } \\
\text { indigestion; vomiting; diarrhoea; gonorrhea; hydrophobia; } \\
\text { joint pains; promotes lactation; insecticidal; wormicidal; } \\
\text { gynaecological disorders; jaundice; syphilis; asthma; } \\
\text { abortifacient; aphrodisiac; tuberculosis; galactogogue; } \\
\text { impotency; foetal health; spider bite; dysentery }\end{array}$ & Foetus & $\begin{array}{l}\text { Dey and De 2012, Dhal et al. } \\
2011 \text {, Madhu and Swamy 2010, } \\
\text { Xavier et al. } 2014\end{array}$ \\
\hline \multicolumn{6}{|c|}{ 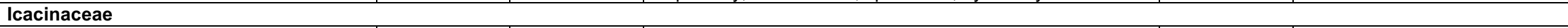 } \\
\hline $\begin{array}{l}\text { Nothapodytes nimmoniana (J. Graham) } \\
\text { Mabb. }\end{array}$ & $\begin{array}{l}\text { Shrub to } \\
\text { small tree }\end{array}$ & Leaves & Psoriasis; Scabies; cancerous wound; epilepsy & General & Prasad et al. 2013 \\
\hline \multicolumn{6}{|l|}{ Iridaceae } \\
\hline Crocus sativus L. & Herb & Flower & - & General & $\begin{array}{l}\text { Chopda and Mahajan 2009, } \\
\text { Dansi et al. 2008, Jain et al. } \\
2009\end{array}$ \\
\hline Iris × germanica L. & Herb & Stem & - & General & Chopda and Mahajan 2009 \\
\hline \multicolumn{6}{|l|}{ Juglandaceae } \\
\hline Juglans regia L. & Tree & $\begin{array}{l}\text { Leaves, } \\
\text { fruit, root, } \\
\text { bark }\end{array}$ & Sore throat & General & Kayani et al. 2014 \\
\hline \multicolumn{6}{|l|}{ Lamiaceae } \\
\hline Ajuga $\times$ adulterina Wallr. & Herb & Whole plant & Throat infection; tonsillitis & General & Kayani et al. 2014 \\
\hline Anisochilus carnosus (L.f.) Wall. & Herb & Aerial parts & $\begin{array}{l}\text { To eliminate the ticks of the domestic dogs; deep cut by } \\
\text { iron; cough; stomachache; fever; chronic blisters; evil } \\
\text { spirits }\end{array}$ & General & RamaChandran et al. 2009 \\
\hline Anisomeles indica (L.) Kuntze & Herb & Whole plant & $\begin{array}{l}\text { Rheumatism; fever; snake bite; epilepsy; Astringent; } \\
\text { sores; ulcers; cough; dyspepsia; malarial fevers; chest } \\
\text { congestion; bronchial troubles }\end{array}$ & General & $\begin{array}{l}\text { Padal et al. 2014, Rao et al. } \\
2006\end{array}$ \\
\hline
\end{tabular}

Published 11 February 2019

http://dx.doi.org/10.32859/era.18.4.1-112 


\begin{tabular}{|c|c|c|c|c|c|}
\hline Anisomeles malabarica (L.) R. Br. ex Sims & Shrub & Whole plant & $\begin{array}{l}\text { Snakebite; carminative; astringent; stomachic; } \\
\text { rheumatism; fever; colic dyspepsia and catarrhal } \\
\text { affections; wounds; anti-inflammatory and anti-pyretic; } \\
\text { dysentery }\end{array}$ & General & $\begin{array}{l}\text { Dey and De 2012, Ramya 2008, } \\
\text { Valsaraj et al. } 1997\end{array}$ \\
\hline Callicarpa arborea Roxb. & Tree & Stem, bark & Cuts and wound healing & General & Kumar et al. 2007 \\
\hline Callicarpa tomentosa (L.) L. & $\begin{array}{l}\text { Shrub to } \\
\text { small tree }\end{array}$ & Leaves & Skin boils; eczema & General & Bhat et al. 2014 \\
\hline Clerodendrum chinense (Osbeck) Mabb. & Shrub & Seed & Rheumatism; constipation & General & Rao et al. 2006 \\
\hline Clerodendrum glandulosum Lindl. & Shrub & Leaves & High blood pressure; stomach disorder & General & Ruba and Mohan 2016 \\
\hline Clerodendrum infortunatum $\mathrm{L}$. & $\begin{array}{l}\text { Shrub to } \\
\text { small tree }\end{array}$ & Leaves, root & $\begin{array}{l}\text { Wounds and cuts; scabies; skin disease; antibaterial; } \\
\text { diarrhoea; dysentery; food poisoning; effective against } \\
\text { tape worm; hook worm infections; amoebic dysentery; for } \\
\text { easy delivery }\end{array}$ & $\begin{array}{l}\text { General; } \\
\text { pregnant } \\
\text { women }\end{array}$ & $\begin{array}{l}\text { Bhat et al. 2014, Harsha et } \\
\text { al.2003, Pushpangadan and Atal } \\
1984\end{array}$ \\
\hline Clerodendrum phlomidis L.f. & Shrub & Leaves & Rheumatism; headache & General & Sreeramulu et al. 2013 \\
\hline Clerodendrum viscosum Vent. & $\begin{array}{l}\text { Shrub to } \\
\text { small tree }\end{array}$ & $\begin{array}{l}\text { Root, aerial } \\
\text { parts }\end{array}$ & $\begin{array}{l}\text { Epilepsy; rheumatism; menorrhegia; tonsilitis; scorpion } \\
\text { stings; skin disease }\end{array}$ & $\begin{array}{l}\text { General; } \\
\text { women }\end{array}$ & $\begin{array}{l}\text { Chander et al. 2015, Sharma et } \\
\text { al. } 2013\end{array}$ \\
\hline Clerodendrum volubile P. Beauv. & Liana & Leaves & Constipation & Cattle & Sharma and Mujundar 2003 \\
\hline Clinopodium umbrosum (M. Bieb.) Kuntze & Herb & Whole plant & $\begin{array}{l}\text { Relieves pain and set the stomach and uterus in right } \\
\text { position. }\end{array}$ & Women & Pushpangadan and Atal 1984 \\
\hline Colebrookea oppositifolia Sm. & Shrub & Root, leaves & A remedy for epilepsy; wound; cuts & General & Sharma et al. 2013 \\
\hline Endostemon viscosus (Roth) M.R. Ashby & Shrub & Whole plant & Skin diseases and to prevent falling of hair & General & Ignacimuthu et al. 2008 \\
\hline Gmelina arborea Roxb. & $\begin{array}{l}\text { Medium } \\
\text { sized tree }\end{array}$ & $\begin{array}{l}\text { Leaves, } \\
\text { wood, bark, } \\
\text { fruit, root, } \\
\text { stem }\end{array}$ & $\begin{array}{l}\text { Bone fracture; bone strengthening; pain relieving and } \\
\text { inflammation reduction; piles; scorpion bite; cardiac } \\
\text { disorders; antipyretic; stomachache; eczema; vegetable; } \\
\text { tinea pedis; wound; impotency }\end{array}$ & $\begin{array}{l}\text { General; } \\
\text { women; } \\
\text { men }\end{array}$ & $\begin{array}{l}\text { Dansi et al. 2008, Ramya 2008, } \\
\text { Ratnam and Raju } 2008\end{array}$ \\
\hline Gmelina asiatica L. & $\begin{array}{l}\text { Shrub to } \\
\text { small tree }\end{array}$ & $\begin{array}{l}\text { Fruit, } \\
\text { leaves, root }\end{array}$ & $\begin{array}{l}\text { Eczema; epistaxis; used as soap; dandruff; throat } \\
\text { infection; cough; diabetes }\end{array}$ & General & $\begin{array}{l}\text { Ragupathy and Newmaster } \\
2009 \text {, Sreeramulu et al. } 2013\end{array}$ \\
\hline Gmelina elliptica Sm. & $\begin{array}{l}\text { Shrub to } \\
\text { small tree }\end{array}$ & Root & Blood purifier & General & Prasad et al. 2008 \\
\hline $\begin{array}{l}\text { Gomphostemma heyneanum Wall. ex } \\
\text { Benth. }\end{array}$ & Herb & Leaves & Dysentery; diarrhea & General & Prasad et al. 2013 \\
\hline $\begin{array}{l}\text { Gomphostemma javanicum (Blume) } \\
\text { Benth. }\end{array}$ & Herb & Leaves & Skin diseases & General & Prasad et al. 2008 \\
\hline Hoslundia opposita Vahl & Shrub & Leaves & Vegetable & General & Dansi et al. 2008 \\
\hline Hyptis lanceolata Poir. & Herb & Leaves & Vegetable & General & Dansi et al. 2008 \\
\hline Hyptis suaveolens (L.) Poit. & Shrub & Whole plant & $\begin{array}{l}\text { Rheumatic pain; wound; gastrointestinal disorders; } \\
\text { respiratory tract and uterus infections; skin diseases; } \\
\text { cancers; tumours; parasitical; cutaneous; headache; } \\
\text { stomach-ache; nose bleeding; leuchorrea; antidiarrhoeal; } \\
\text { fever }\end{array}$ & General & $\begin{array}{l}\text { Dey and De 2012, Lingaraju et } \\
\text { al. 2013, Padal et al. 2010, } \\
\text { Padal et al. } 2014\end{array}$ \\
\hline Isodon rubescens (Hemsl.) H. Hara & Herb & - & Anti-inflammatory & General & Zhang et al. 2013 \\
\hline Isodon rugosus (Wall. ex Benth.) Codd & Shrub & Leaves & - & General & Dey and De 2012 \\
\hline Lavandula stoechas L. & Shrub & $\begin{array}{l}\text { Flower } \\
\text { heads }\end{array}$ & To relieve blue spots due to knocks & General & Parada et al. 2009 \\
\hline
\end{tabular}

Published 11 February 2019

http://dx.doi.org/10.32859/era.18.4.1-112 


\begin{tabular}{|c|c|c|c|c|c|}
\hline Leonotis nepetifolia (L.) R. Br. & Shrub & Whole plant & $\begin{array}{l}\text { Paralysis; skin disease; rheumatism; post natal swelling; } \\
\text { regulates periods; diarrhoea; febrifuge; scalds; burns; } \\
\text { wound; night blindness; eczema; malaria; antihelminthic; } \\
\text { scorpion sting; jaundice; sores; pre natal vomiting; urinary } \\
\text { diseases; intoxication; fever; epilepsy; asthma; anticancer }\end{array}$ & General & $\begin{array}{l}\text { Kumar et al. 2007, } \\
\text { Prashantkumar and Vidyasagar } \\
\text { 2008, Wagh and Jain } 2015\end{array}$ \\
\hline Leonurus japonicus Houtt. & Herb & - & Anti-tumour & General & Zhang et al. 2013 \\
\hline Leonurus sibiricus L. & Herb & Whole plant & Leucorrhoea & General & Lingaraju et al. 2013 \\
\hline Leucas aspera (Willd.) Link & Herb & Whole plant & $\begin{array}{l}\text { Dysentery; headache; fever; toothache; asthma; cold; skin } \\
\text { eruptions; nasal congestion; snake bite; ulcers; scabies; } \\
\text { scorpion bite; bronchitis; psoriasis; swelling; rheumatism; } \\
\text { skin problems; jaundice; antibacterial; throat infection; } \\
\text { abortifacient; diabetes; gastrointestinal disorders; oral } \\
\text { health care; anthelmintic; gum infection }\end{array}$ & $\begin{array}{l}\text { Children; } \\
\text { cattle; } \\
\text { general }\end{array}$ & $\begin{array}{l}\text { Harsha et al. 2003, Kshirsagar } \\
\text { and Singh 2001, Lingaraju et al. } \\
\text { 2013, Raghupathy and } \\
\text { Newmaster 2009, Sharma et al. } \\
2013\end{array}$ \\
\hline Leucas biflora (Vahl) Sm. & Herb & Whole plant & Skin irritations; headache; fever & General & $\begin{array}{l}\text { Deepak and Gopal 2014, } \\
\text { Ignacimuthu et al. } 2013\end{array}$ \\
\hline Leucas cephalotes (Roth) Spreng. & Herb & Whole plant & Dermatitis; snakebite & General & Samy et al. 2008 \\
\hline Leucas lanata Benth. & Herb & Leaves & Wound healing; anti-microbial; anti-oxidant & General & Kanthasamy Kalaichelvi 2016 \\
\hline Leucas lavandulifolia Sm. & Herb & $\begin{array}{l}\text { Leaves, } \\
\text { flowers }\end{array}$ & Arthritis; snake bite & General & Dey and De 2012 \\
\hline Leucas pubescens Benth. & $\begin{array}{l}\text { Prostrate } \\
\text { branching } \\
\text { herb }\end{array}$ & Leaves & Antipyretic; antifungal; antimicrobial & General & Dhivya and Kalaichelvi 2015 \\
\hline Leucas urticifolia (Vahl) Sm. & Herb & Whole plant & Asthma; fever; astringent bitter & General & Kanthasamy Kalaichelvi 2016 \\
\hline Leucas zeylanica (L.) W.T. Aiton & Herb & Leaves & Scorpion sting & General & Savithramma et al. 2014 \\
\hline Lycopus europaeus $\mathrm{L}$. & Herb & Leaves & - & - & Chopda and Mahajan 2009 \\
\hline Marrubium vulgare $\mathrm{L}$. & Subshrub & Aerial parts & For stones in the kidney or in the bile bladder & General & Parada et al. 2009 \\
\hline Mentha arvensis L. & Creeper & $\begin{array}{l}\text { Stem, } \\
\text { leaves }\end{array}$ & $\begin{array}{l}\text { Paralysis; headache; toothache; congestive disorder; } \\
\text { stomachic; hyperacidity }\end{array}$ & General & $\begin{array}{l}\text { Lingaraju et al. 2013, Prashanth } \\
\text { Kumar and Shiddamallayya } \\
2015\end{array}$ \\
\hline Mentha spicata L. & Herb & Leaves & $\begin{array}{l}\text { Digestive; for stomach ache; to stabilize the belly; scabies; } \\
\text { intestinal worms }\end{array}$ & General & $\begin{array}{l}\text { Kosalge and Fursule 2009, } \\
\text { Parada et al. } 2009\end{array}$ \\
\hline Meriandra strobilifera Benth. & Shrub & Leaves & - & General & Chopda and Mahajan 2009 \\
\hline Ocimum americanum $\mathrm{L}$. & Herb & Whole plant & $\begin{array}{l}\text { Malaria; fever; digestive; cardio tonic; cold; cough; fever; } \\
\text { pain; ear infections; skin eruptions; boils; fumigation; } \\
\text { bronchitis; increases sexual potency; insecticidal; } \\
\text { wormicidal; dandruff; gastrointestinal problems }\end{array}$ & General & $\begin{array}{l}\text { Ayyanar and Ignacimuthu 2005, } \\
\text { Bosco and Arumugam } 2012\end{array}$ \\
\hline Ocimum basilicum $\mathrm{L}$. & Herb & Whole plant & $\begin{array}{l}\text { Bone fracture; antipyretic; diaphoretic; chronic } \\
\text { constipation; diuretic; dysentery; cold; cough; fever; } \\
\text { malaria; stomachache; earache; insect bites; scars; skin } \\
\text { rashes; corneal opacity; pimples; insecticidal; wormicidal; } \\
\text { urinary problems; vermifuge }\end{array}$ & General & $\begin{array}{l}\text { Ayyanar and Ignacimuthu } 2005 \text {, } \\
\text { Lingaraju et al. 2013, Padma et } \\
\text { al. } 2016\end{array}$ \\
\hline Ocimum filamentosum Forssk. & Herb & Whole plant & Snakebite & General & Dey and De 2012 \\
\hline Ocimum gratissimum L. & Shrub & Whole plant & $\begin{array}{l}\text { Rheumatism; diuretic; anti septic; cough; head ache; } \\
\text { dysentery; urinary disorders; breathing problems; seminal }\end{array}$ & General & $\begin{array}{l}\text { Dansi et al. 2008, Savithramma } \\
\text { et al. 2014, Sharma et al. } 2013\end{array}$ \\
\hline
\end{tabular}

Published 11 February 2019

http://dx.doi.org/10.32859/era.18.4.1-112 


\begin{tabular}{|c|c|c|c|c|c|}
\hline & & & $\begin{array}{l}\text { weakness; fever; vegetable; cough; typhoid; skin } \\
\text { infections; health tonic }\end{array}$ & & \\
\hline Ocimum tenuiflorum $\mathrm{L}$. & Herb & Whole plant & $\begin{array}{l}\text { Bronchitis; asthma; malaria; diarrhoea; dysentery; skin } \\
\text { diseases; arthritis; fever; insect bite; indigestion; cough; } \\
\text { cold; hysteria; headache; unconsciousness; cholera; } \\
\text { abortion; fits; scorpion sting; jaundice; malaria; tumor; } \\
\text { bronchitis; constipation; piles; anti diabetic; tuberculosis; } \\
\text { increases sperm count; wound; cuts; snakebite; scorpion } \\
\text { sting }\end{array}$ & $\begin{array}{l}\text { General; } \\
\text { children; } \\
\text { pregnant } \\
\text { women; } \\
\text { men }\end{array}$ & $\begin{array}{l}\text { Dey et al. 2012, Hebbar et al. } \\
\text { 2004, Harsha et al. 2003, Jain } \\
\text { 2004, Mahishi et al. } 2005\end{array}$ \\
\hline Origanum vulgare $\mathrm{L}$. & Herb & Flower & Good for everything & General & Parada et al. 2009 \\
\hline Orthosiphon thymiflorus (Roth) Sleesen & Herb & Leaves & Wound healing; ulcer; skin eruptions & General & Kanthasamy Kalaichelvi 2016 \\
\hline Platostoma africanum P. Beauv. & Herb & Leaves & Vegetable & General & Dansi et al. 2008 \\
\hline Plectranthus amboinicus (Lour.) Spreng. & Shrub & $\begin{array}{l}\text { Aerial parts, } \\
\text { root }\end{array}$ & Skin allergy; epilepsy; wound; skin diseases & General & $\begin{array}{l}\text { Harsha et al. 2003, Lingaraju et } \\
\text { al. } 2013\end{array}$ \\
\hline Plectranthus barbatus Andrews & Herb & Leaves & Eye problems; for reduced eye pressure; weight loss & General & Jayanthi et al. 2012 \\
\hline Plectranthus fruticosus L'Hér. & Shrub & Leaves & For boils and blisters & General & Rajendran 2000 \\
\hline Plectranthus glabratus (Benth.) Alston & Shrub & Leaves & Skin disease; burns; body pain & General & Ramya 2008 \\
\hline $\begin{array}{l}\text { Plectranthus malabaricus (Benth.) R.H. } \\
\text { Willemse }\end{array}$ & Herb & Leaves & Asthma & General & Puravankara and Gopal 2012 \\
\hline Plectranthus mollis (Aiton) Spreng. & Herb & Whole plant & Wounds & General & Sreeramulu et al. 2013 \\
\hline Plectranthus rotundifolius (Poir.) Spreng. & Herb & Tuber & Itching; boils on the skin & General & Puravankara and Gopal 2012 \\
\hline Plectranthus scutellarioides (L.) R. Br. & Herb & - & Applied on skin during scorpion bite & General & Namsa et al. 2009 \\
\hline Pogostemon auricularius (L.) Hassk. & Herb & $\begin{array}{l}\text { Leaves, } \\
\text { stem }\end{array}$ & Cough & General & Gailea et al. 2016 \\
\hline $\begin{array}{l}\text { Pogostemon benghalensis (Burm. f.) } \\
\text { Kuntze }\end{array}$ & Shrub & Whole plant & Wounds; fever & General & Kumar et al. 2007 \\
\hline Pogostemon cablin (Blanco) Benth. & Herb & - & Asthma; antifungal & General & Zhang et al. 2013 \\
\hline Pogostemon myosuroides (Roth) Kuntze & Herb & Leaves & Blood purifier & General & Savithramma et al. 2014 \\
\hline Premna mollissima Roth & $\begin{array}{l}\text { Shrub to } \\
\text { small tree }\end{array}$ & Bark, leaves & Ringworm; oedema & General & Ram et al. 2004 \\
\hline Premna serratifolia L. & $\begin{array}{l}\text { Shrub to } \\
\text { small tree }\end{array}$ & - & Cardiac disorders & General & Chopda and Mahajan 2004 \\
\hline Premna tomentosa Willd. & $\begin{array}{l}\text { Moderate } \\
\text { size tree }\end{array}$ & Bark, fruit & Stomach disorders; edible & General & Savithramma et al. 2014 \\
\hline Prunella vulgaris $\mathrm{L}$. & Herb & Whole plant & $\begin{array}{l}\text { To disinfect wounds; antibacterial; respiratory difficulties; } \\
\text { cough }\end{array}$ & General & Zhang et al. 2013 \\
\hline Rosmarinus officinalis $\mathrm{L}$. & Herb & - & $\begin{array}{l}\text { To calm pain and deflate knocks; for colds; for knocks; for } \\
\text { bones and joints pain; to wash wounds; for joints and } \\
\text { knees pain; it helps to digest; to enhance circulation; for } \\
\text { laziness; for muscular pain }\end{array}$ & General & Parada et al. 2009 \\
\hline Rotheca serrata (L.) Steane and Mabb. & $\begin{array}{l}\text { Tree or } \\
\text { rambling } \\
\text { shrub }\end{array}$ & Leaves, root & $\begin{array}{l}\text { Typhoid; rheumatoid arthritis; cancer; malaria; stomach } \\
\text { upset; labour pain; high fever; cough rheumatism; } \\
\text { fractures; antibacterial; skin disease; asthma; piles }\end{array}$ & $\begin{array}{l}\text { General; } \\
\text { pregnant } \\
\text { women }\end{array}$ & $\begin{array}{l}\text { Ignacimuthu et al. 2008, Padal } \\
\text { et al. 2014, Rao et al. 2006, } \\
\text { Valsarai et al. } 1997\end{array}$ \\
\hline Salvia canariensis $\mathrm{L}$. & Shrub & Flower, root & Cough; cold & General & Kayani et al. 2014 \\
\hline Salvia miltiorrhiza Bunge & Small shrub & - & Anti-tumour & General & Zhang et al. 2013 \\
\hline
\end{tabular}

Published 11 February 2019

http://dx.doi.org/10.32859/era.18.4.1-112 
Ethnobotany Research and Applications

\begin{tabular}{|c|c|c|c|c|c|}
\hline Salvia moorcroftiana Wall. ex Benth. & Herb & $\begin{array}{l}\text { Leaves, } \\
\text { seed, root }\end{array}$ & Cough; cold & General & Chopda and Mahajan 2009 \\
\hline Salvia officinalis $\mathrm{L}$. & Subshrub & Leaves & For menopause problems; diabetes & $\begin{array}{l}\text { General; } \\
\text { women }\end{array}$ & Parada et al. 2009 \\
\hline Scutellaria baicalensis Georgi & Herb & - & Anti-tumour & General & Zhang et al. 2013 \\
\hline Scutellaria barbata D. Don & Herb & - & Anti-microbial & General & Zhang et al. 2013 \\
\hline Scutellaria violacea B. Heyne ex Benth. & Herb & $\begin{array}{l}\text { Flower, } \\
\text { leaves }\end{array}$ & Asthma; cough and fever & General & Dhivya and Kalaichelvi 2015 \\
\hline $\begin{array}{l}\text { Sideritis sventenii (G. Kunkel) Mend.- } \\
\text { Heuer }\end{array}$ & Shrub & Leaves & For troubles linked to wounds & General & Parada et al. 2009 \\
\hline $\begin{array}{l}\text { Sphenodesme involucrata (C. Presl) B.L. } \\
\text { Rob. }\end{array}$ & Climber & Leaves & Body pain & General & Binu 2011 \\
\hline Symphorema polyandrum Wight & $\begin{array}{l}\text { Climbing } \\
\text { shrub }\end{array}$ & Seed, root & Snake bite & General & Nanjunda 2010 \\
\hline Tectona grandis L.f. & Tree & Whole plant & $\begin{array}{l}\text { Ovulation enhancement; skin diseases; cholera; cough; } \\
\text { ulcers; filariasis; ringworm; dysmennorhea; kidney stone; } \\
\text { headache; leprosy; leucorrhea; skin burns; to ease child } \\
\text { birth; to mitigate abdominal pain during labour }\end{array}$ & $\begin{array}{l}\text { General; } \\
\text { pregnant } \\
\text { women; } \\
\text { women }\end{array}$ & $\begin{array}{l}\text { Jain } 2004 \text {, Jain et al. } 2004, \\
\text { Kshirsagar and Singh } 2001, \\
\text { Sreeramulu et al. } 2013\end{array}$ \\
\hline Thymus mongolicus (Ronniger) Ronniger & Herb & Whole plant & Cough; cold; flu; sore throat; bronchitis; chest infections & General & Sharma et al. 2013 \\
\hline Thymus vulgaris $\mathrm{L}$. & Herb & Whole plant & $\begin{array}{l}\text { Wound; bellyache; mouth sores; sore throat; sore eyes; } \\
\text { laxative; toothache; gums inflammation; cold; urine } \\
\text { infection; snakebite }\end{array}$ & $\begin{array}{l}\text { General; } \\
\text { women }\end{array}$ & $\begin{array}{l}\text { Parada et al. 2009, Samy et al. } \\
2008\end{array}$ \\
\hline Vitex agnus-castus $\mathrm{L}$. & Shrub & $\begin{array}{l}\text { Flowers, } \\
\text { leaves }\end{array}$ & For warts; to low sugar & General & Parada et al. 2009 \\
\hline Vitex altissima L.f. & Tree & Root, bark & Wounds; snakebite & General & Dey and De 2012 \\
\hline Vitex doniana Sweet & Tree & Leaves & Vegetable & General & Dansi et al. 2008 \\
\hline Vitex leucoxylon L.f. & Tree & Bark, leaves & Leprosy; headache; antibacterial & General & Valsaraj et al. 1997 \\
\hline Vitex negundo L. & $\begin{array}{l}\text { Shrub to } \\
\text { small tree }\end{array}$ & Whole plant & $\begin{array}{l}\text { Abortifacient; epilepsy; antibacterial; antidiarrhoeal; } \\
\text { asthma; headache; cold; cough; wound; diabetes; cardiac } \\
\text { disorders; intestinal worms; fever; spleen disorder; oral } \\
\text { health care; mouth sores; repells mosquitoes; rheumatism; } \\
\text { retained placenta; insecticide; increases lactation; goiter; } \\
\text { epilepsy; typhoid }\end{array}$ & $\begin{array}{l}\text { General; } \\
\text { women; } \\
\text { pregnant } \\
\text { women }\end{array}$ & $\begin{array}{l}\text { Ramya 2008, Samy et al. 2008, } \\
\text { Sreeramulu et al. 2013, Tetali et } \\
\text { al. 2009, Valsaraj et al. } 1997\end{array}$ \\
\hline Vitex peduncularis Wall. ex Schauer & Tree & $\begin{array}{l}\text { Bark, aerial } \\
\text { parts, stem, } \\
\text { leaves }\end{array}$ & $\begin{array}{l}\text { Malaria; typhoid; edema; obestric diseases; wound; } \\
\text { antiinflammatory; wound }\end{array}$ & General & Kanthasamy Kalaichelvi 2016 \\
\hline Vitex trifolia L. & Shrub & Whole plant & Hair fall; malaria; rheumatic swelling & General & Mahishi et al. 2005 \\
\hline Volkameria inermis L. & Shrub & $\begin{array}{l}\text { Leaves, } \\
\text { root, stem }\end{array}$ & $\begin{array}{l}\text { Anthelmintic; diabetes; snake bite; malaria; clot blood in } \\
\text { cut wounds; swellings; pains and inflammation; diabetes }\end{array}$ & General & $\begin{array}{l}\text { Mahishi et al. 2005, SM and } \\
\text { Vidyasagar } 2013, \text { Sripathi and } \\
\text { Sankari } 2010\end{array}$ \\
\hline \multicolumn{6}{|l|}{ Lardizabalaceae } \\
\hline Akebia quinata (Houtt.) Decne. & Climber & - & Anti-bacterial & General & Zhang et al. 2013 \\
\hline \multicolumn{6}{|l|}{ Lauraceae } \\
\hline Cassytha filiformis $\mathrm{L}$. & Climber & Whole plant & $\begin{array}{l}\text { Jaundice; Bone fracture; scorpion stings; ulcers; } \\
\text { hydrocele; rheumatism }\end{array}$ & General & $\begin{array}{l}\text { Al-Asmari et al. 2016, Rao et al. } \\
2006\end{array}$ \\
\hline
\end{tabular}

Published 11 February 2019

http://dx.doi.org/10.32859/era.18.4.1-112 


\begin{tabular}{|c|c|c|c|c|c|}
\hline Cinnamomum camphora (L.) J. Presl & Tree & Latex & Rheumatoid arthritis & General & Arjunan et al. 2016 \\
\hline Cinnamomum iners Reinw. ex Blume & Tree & Bark & Jaundice & General & Lingaraju et al. 2013 \\
\hline $\begin{array}{l}\text { Cinnamomum malabatrum (Burm. f.) J. } \\
\text { Presl }\end{array}$ & Tree & Bark & Rheumatism & General & $\begin{array}{l}\text { Prashanth Kumar and } \\
\text { Shiddamallayya } 2015\end{array}$ \\
\hline $\begin{array}{l}\text { Cinnamomum tamala (Buch. -Ham.) T. } \\
\text { Nees and Eberm. }\end{array}$ & Small tree & Bark & $\begin{array}{l}\text { Indigestion; fever; nourishment; chicken pox; vomiting; } \\
\text { cough }\end{array}$ & General & Chander et al. 2015 \\
\hline Cinnamomum verum J. Presl & Tree & Bark & $\begin{array}{l}\text { Diabetes mellitus; rheumatoid arthritis; antiallergic; } \\
\text { antiulcerogenic; antipyretic and anaesthetic; infertility in } \\
\text { male; boils; edible }\end{array}$ & $\begin{array}{l}\text { General; } \\
\text { men }\end{array}$ & $\begin{array}{l}\text { Bhat et al. 2014, Das et al. } \\
\text { 2013, Mallikarjuna } 2015\end{array}$ \\
\hline Cinnamomum wightii Meisn. & Tree & Bark & Expectorant; bone fracture & General & Upadhya et al. 2012 \\
\hline Lindera neesiana (Wall. ex Nees) Kurz & Shrub & Fruit, seed & Anthelmintic; diarrhoea; scabies; vegetable oils & General & Ruba and Mohan 2016 \\
\hline Litsea cubeba (Lour.) Pers. & Tree & Fruit & Condiments; eczema; heart disease and stomach disorder & General & Ruba and Mohan 2016 \\
\hline Litsea deccanensis Gamble & Tree & Bark, leaves & Body pains; scabies; hard boils; testis in pains & $\begin{array}{l}\text { Men; } \\
\text { general }\end{array}$ & Rao et al. 2006, Rao et al. 2011 \\
\hline Litsea floribunda Gamble & Tree & Bark & Malarial treatment & General & Lingaraju et al. 2013 \\
\hline Litsea glutinosa (Lour.) C.B. Rob. & Tree & Whole plant & Labour pains; bone fracture; boils; wound & General & Kumar et al. 2007 \\
\hline Litsea ligustrina (Nees) Fern. -Vill. & Tree & $\begin{array}{l}\text { Leaves, } \\
\text { bark, flower }\end{array}$ & Snake and scorpion bites & General & Dey and De 2012 \\
\hline Litsea scrobiculata Meisn. & Tree & Bark, leaves & Diarrhea; dysentery; fractured limbs & General & Kanthasamy Kalaichelvi 2016 \\
\hline Persea americana Mill. & Tree & Fruit & Edible & General & Rekka and Kumar 2014 \\
\hline Persea macrantha (Nees) Kosterm. & Tree & Stem, bark & Bone fracture and dislocation & General & Upadhya et al. 2004 \\
\hline \multicolumn{6}{|l|}{ Lecythidaceae } \\
\hline Barringtonia acutangula (L.) Gaertn. & Tree & $\begin{array}{l}\text { Bark, } \\
\text { leaves, fruit }\end{array}$ & Rheumatoid arthritis; rheumatism; dysentery; lactation & $\begin{array}{l}\text { General, } \\
\text { women, } \\
\text { cattle }\end{array}$ & Mallikarjuna 2015 \\
\hline Barringtonia racemosa (L.) Spreng. & Tree & $\begin{array}{l}\text { Aerial parts, } \\
\text { bark }\end{array}$ & $\begin{array}{l}\text { Dog bite; snakebite; snake repellent; anorexia; skin allergy } \\
\text { septic }\end{array}$ & General & $\begin{array}{l}\text { Bhat et al. 2012, Dey and De } \\
2012\end{array}$ \\
\hline Careya arborea Roxb. & Tree & $\begin{array}{l}\text { Bark, flower, } \\
\text { fruit }\end{array}$ & $\begin{array}{l}\text { Tooth ache; debility; labour pains; infertility; piles; scorpion } \\
\text { sting; dysentery; leech repellent; coughand cold; snake } \\
\text { bite; sores; malaria }\end{array}$ & General & $\begin{array}{l}\text { Bhat et al. 2014, Kshirsagar and } \\
\text { Singh 2001, Mahishi et al. } 2005\end{array}$ \\
\hline Couroupita guianensis Aubl. & Tree & $\begin{array}{l}\text { Leaves, } \\
\text { fruit, flower }\end{array}$ & $\begin{array}{l}\text { Malaria; skin diseases; toothache; anthelmintic; cold; } \\
\text { stomachache }\end{array}$ & General & Alagesaboopathi 2012 \\
\hline Planchonia valida (Blume) Blume & Tree & Root & Diarrhoea & General & Prasad et al. 2008 \\
\hline \multicolumn{6}{|l|}{ Liliaceae } \\
\hline Cardiocrinum giganteum (Wall.) Makino & Herb & Leaves & - & General & $\begin{array}{l}\text { Chopda and Mahajan 2009, } \\
\text { Srithi et al. } 2009\end{array}$ \\
\hline Tulipa albanica Kit Tan and Shuka & $\begin{array}{l}\text { Tree and } \\
\text { shrub }\end{array}$ & Tuber & Bone fracture & General & Bhuvaneswari et al. 2015 \\
\hline Hugonia serrata Lam. & Herb & $\begin{array}{l}\text { Leaves, } \\
\text { bark }\end{array}$ & $\begin{array}{l}\text { Toothache; inflammations in the stomach; vomiting; } \\
\text { stomach pain; indigestion; snake bite; dysentery }\end{array}$ & General & Pushpangadan and Atal 1984 \\
\hline Linum usitatissimum L. & Herb & Seed, oil & - & General & Chopda and Mahajan 2009 \\
\hline \multicolumn{6}{|l|}{ Linderniaceae } \\
\hline Lindernia caespitosa (Blume) Panigrahi & $\begin{array}{l}\text { Prostrate or } \\
\text { diffuse herb }\end{array}$ & Whole plant & Fever; cough; cold; antiinflammatory & General & Dhivya and Kalaichelvi 2015 \\
\hline
\end{tabular}

Published 11 February 2019

http://dx.doi.org/10.32859/era.18.4.1-112 


\begin{tabular}{|c|c|c|c|c|c|}
\hline $\begin{array}{l}\text { Mitreola petiolata (J.F. Gmel.) Torr. and A. } \\
\text { Gray }\end{array}$ & Herb & Leaves & Urinary disorder & General & Wagh and Jain 2015 \\
\hline Spigelia anthelmia L. & Forb/herb & Leaves & Anthelmintic; vegetable & General & Wagh and Jain 2015 \\
\hline Strychnos nux-vomica L. & Tree & Seed & $\begin{array}{l}\text { Abortion; diarrhoea; rheumatism; piles; dysentery; arthritis; } \\
\text { boils; cough; facial paralysis; leucoderma; insect bite; post } \\
\text { natal problems; snakebite; spermatorrhea }\end{array}$ & $\begin{array}{l}\text { General; } \\
\text { men; } \\
\text { pregnant } \\
\text { women }\end{array}$ & Parijatham and Sujatha 2016 \\
\hline Strychnos potatorum L.f. & Tree & Whole plant & $\begin{array}{l}\text { Scorpion sting; snake bite; peptic ulcers; urinary and } \\
\text { kidney problems }\end{array}$ & General & $\begin{array}{l}\text { Padma et al. 2016, Sharma et } \\
\text { al. } 2013\end{array}$ \\
\hline Strychnos spinosa Lam. & Tree & Leaves & Vegetable & General & Dansi et al. 2008 \\
\hline Strychnos wallichiana Steud. ex A. DC. & Tree & Bark & Malarial treatment & General & Chander et al. 2015 \\
\hline \multicolumn{6}{|l|}{ Loranthaceae } \\
\hline Dendrophthoe falcata (L.f.) Ettingsh. & Herb & Whole plant & $\begin{array}{l}\text { Astringent; aphrodiasic; narcotic; diuretic; tuberculosis; } \\
\text { asthma; menstrual disorders; swelling wounds; ulcers; } \\
\text { renal and vesical calculi; antifertility agent; anticancerous; } \\
\text { skin diseases; asthma; abortion; migrane; muscle pains }\end{array}$ & $\begin{array}{l}\text { General; } \\
\text { women; } \\
\text { pregnant } \\
\text { women }\end{array}$ & $\begin{array}{l}\text { Lingaraju et al. 2013, } \\
\text { Ragupathy and Newmaster } \\
\text { 2009, Sharma et al. } 2013\end{array}$ \\
\hline Loranthus longiflorus Desr. & Tree & $\begin{array}{l}\text { Leaves, } \\
\text { flower }\end{array}$ & Antioxidant & General & Kanthasamy Kalaichelvi 2016 \\
\hline $\begin{array}{l}\text { Phragmanthera kamerunensis (Engl.) } \\
\text { Balle }\end{array}$ & Shrub & Leaves & Vegetable & General & Dansi et al. 2008 \\
\hline Scurrula parasitica L. & Herb & Leaves & Reduce swelling; lower blood pressure; liver diseases & General & Kanthasamy Kalaichelvi 2016 \\
\hline Scurrula stocksii Danser & Herb & Fruit & Edible & General & Bhagat et al. 2016 \\
\hline Taxillus chinensis (DC.) Danser & $\begin{array}{l}\text { Parasitic } \\
\text { shrub }\end{array}$ & - & Anti-virus & General & Zhang et al. 2013 \\
\hline \multicolumn{6}{|l|}{ Lycopodiaceae } \\
\hline Lycopodiella cernua (L.) Pic. Serm. & Ferns & Whole plant & Treating convulsion or voice loss; obstetric diseases & General & Srithi et al. 2009 \\
\hline Lycopodium clavatum L. & Herb & Aerial parts & Muscle pain and fatigue & General & Namsa et al. 2009 \\
\hline \multicolumn{6}{|l|}{ Lygodiaceae } \\
\hline Lygodium flexuosum (L.) Sw. & Ferns & Whole plant & $\begin{array}{l}\text { Contraceptive; abortifacient; rheumatism; sprains; scabies; } \\
\text { eczema; cut; wounds; jaundice; cancer; foot and mouth } \\
\text { diseases in animals; ulcer; respiratory disorders; muscle } \\
\text { sprain; fertility; skin disease; spermatorrohea; } \\
\text { dysmenorrhea; expectorant }\end{array}$ & $\begin{array}{l}\text { General; } \\
\text { women; } \\
\text { men }\end{array}$ & $\begin{array}{l}\text { Chopda and Mahajan 2009, } \\
\text { Rao et al. 2006, Sreeramulu et } \\
\text { al. 2013, Wills and Asha } 2006\end{array}$ \\
\hline \multicolumn{6}{|l|}{ Lythraceae } \\
\hline Ammannia baccifera L. & Herb & Whole plant & $\begin{array}{l}\text { Typhoid; malaria; skin disease; eczema; muscular pain; } \\
\text { snake and scorpion bite; wound }\end{array}$ & General & Ram et al. 2004, Rao et al. 2006 \\
\hline Lagerstroemia hypoleuca Kurz & Tree & Leaves & Abdomen pain & General & Chander et al. 2015 \\
\hline Lagerstroemia microcarpa Hance & Tree & Bark & Skin burns & General & Kshirsagar and Singh 2001 \\
\hline Lagerstroemia parviflora Roxb. & Tree & $\begin{array}{l}\text { Leaves, } \\
\text { bark }\end{array}$ & $\begin{array}{l}\text { Boils; blisters; cuts; scabies; skin diseases; abdominal } \\
\text { pain }\end{array}$ & General & Kosalge and Fursule 2009 \\
\hline Lagerstroemia speciosa (L.) Pers. & Tree & - & Postpartum health & General & Chithra et al. 2016 \\
\hline Lawsonia inermis $\mathrm{L}$. & $\begin{array}{l}\text { Glabrous } \\
\text { shrub/small } \\
\text { tree }\end{array}$ & $\begin{array}{l}\text { Leaves, } \\
\text { seed, } \\
\text { rhizome }\end{array}$ & $\begin{array}{l}\text { Jaundice; pain; removes excess of bile content; foot } \\
\text { cracks; piles; migrane; headache; skin burns; } \\
\text { spermatorrhea; pimples; gonorrhea; hair tonic; }\end{array}$ & General & $\begin{array}{l}\text { Rao et al. 2006, Sharma et al. } \\
\text { 2013, Sreeramulu et al. } 2013\end{array}$ \\
\hline
\end{tabular}

Published 11 February 2019

http://dx.doi.org/10.32859/era.18.4.1-112 


\begin{tabular}{|c|c|c|c|c|c|}
\hline & & & $\begin{array}{l}\text { conjuctivitis; debility; leucoderma; skin diseases; } \\
\text { contraceptive; wound; boils; cuts; swelling }\end{array}$ & & \\
\hline Punica granatum $\mathrm{L}$. & $\begin{array}{l}\text { Shrub or } \\
\text { small tree. }\end{array}$ & Whole plant & $\begin{array}{l}\text { Anti diabetic; epilepsy; antidiarrhoeal; antimicrobial; } \\
\text { dysentery; diarrhoea; stomach pain; gastrointestinal } \\
\text { disorders; tuberculosis; insecticidal; wormicidal; } \\
\text { indigestion }\end{array}$ & General & $\begin{array}{l}\text { Bhardwaj et al. 2011, Padal et } \\
\text { al. } 2014\end{array}$ \\
\hline $\begin{array}{l}\text { Rotala rotundifolia (Buch. -Ham. ex Roxb.) } \\
\text { Koehne }\end{array}$ & $\begin{array}{l}\text { Perennial } \\
\text { aquatic plant }\end{array}$ & Whole plant & Cough & General & Rao et al. 2011 \\
\hline Woodfordia fruticosa (L.) Kurz & Shrub & $\begin{array}{l}\text { Leaves, } \\
\text { flower, stem } \\
\text { bark, fruit } \\
\end{array}$ & $\begin{array}{l}\text { Blood purifier; diarrhoea; leucoderma; leprosy; burning } \\
\text { sensation; antibacterial; dysentery; cuts; wounds; jaundice }\end{array}$ & General & $\begin{array}{l}\text { Dubey et al. 2012, Rao et al. } \\
2011\end{array}$ \\
\hline \multicolumn{6}{|l|}{ Magnoliaceae } \\
\hline Magnolia champaca (L.) Baill. ex Pierre & Tree & $\begin{array}{l}\text { Bark, } \\
\text { leaves, } \\
\text { flower, root }\end{array}$ & $\begin{array}{l}\text { Cold; fever; stomach ache; urinary problems; skin } \\
\text { diseases; purgative; scorpion bite; worm infections; } \\
\text { amennorhea; prevents pregnancy }\end{array}$ & $\begin{array}{l}\text { General; } \\
\text { women }\end{array}$ & $\begin{array}{l}\text { Muralidhara Rao and Pullaiah } \\
2007\end{array}$ \\
\hline Magnolia nilagirica (Zenker) Figlar & Tree & Flowers & - & General & Kennedy 2006 \\
\hline \multicolumn{6}{|l|}{ Malpighiaceae } \\
\hline Aspidopterys tomentosa (Blume) A. Juss. & Tree & Leaves & Eczema & General & Dubey et al. 2012 \\
\hline Hiptage benghalensis (L.) Kurz & $\begin{array}{l}\text { Climbing } \\
\text { liana/large } \\
\text { shrub }\end{array}$ & Leaves & $\begin{array}{l}\text { Narcotics; hepatitis; insecticide; scabies; asthma and } \\
\text { various skin diseases }\end{array}$ & General & $\begin{array}{l}\text { Prabakaran et al. } 2013, \\
\text { Savithramma et al. } 2014\end{array}$ \\
\hline \multicolumn{6}{|l|}{ Malvaceae } \\
\hline Abelmoschus crinitus Wall. & $\begin{array}{l}\text { Perennial } \\
\text { herb }\end{array}$ & Leaves, fruit & Memory power; dysentery & General & Padal et al. 2012 \\
\hline Abelmoschus esculentus (L.) Moench & Forb/herb & Fruit, seed & Cough; difficulty in breathing like Asthma & General & Dansi et al. 2008 \\
\hline Abelmoschus ficulneus (L.) Wight and Arn. & Erect shrub & \begin{tabular}{|l|l|}
- \\
\end{tabular} & Contraceptive & Women & Bhogaonkar and Kadam 2006 \\
\hline Abelmoschus manihot (L.) Medik. & $\begin{array}{l}\text { Perennial } \\
\text { shrub }\end{array}$ & Root & Skin burns & General & Mali and Bhadane 2011 \\
\hline Abelmoschus moschatus Medik. & $\begin{array}{l}\text { Perennial } \\
\text { shrub }\end{array}$ & $\begin{array}{l}\text { Fruit, seed, } \\
\text { mucilage, } \\
\text { root }\end{array}$ & $\begin{array}{l}\text { Treat muscle diseases externally; abdominal pain; } \\
\text { Purgative; aphrodisiac; abortifacient }\end{array}$ & $\begin{array}{l}\text { General; } \\
\text { pregnant } \\
\text { women }\end{array}$ & $\begin{array}{l}\text { Muralidhara Rao and Pullaiah } \\
2007\end{array}$ \\
\hline Abutilon hirtum (Lam.) Sweet & Shrub & Leaves & Leucorrhoea & General & Kshirsagar and Singh 2001 \\
\hline Abutilon indicum (L.) Sweet & Shrub & Whole plant & $\begin{array}{l}\text { Piles; skin diseases; rheumatism; diabetes; jaundice; } \\
\text { dysentery; urinary troubles;; stomach ulcer; malarial } \\
\text { fevers; cough; cold and chest pain; }\end{array}$ & General & $\begin{array}{l}\text { Jain et al. 2004, Kumar and } \\
\text { Bharati 2013, Lingaraju et al. } \\
2013 \text {, Samy et al. } 2008\end{array}$ \\
\hline Abutilon persicum (Burm. f.) Merr. & Shrub & Dry fruits & $\begin{array}{l}\text { To cure infections and obstructions in the genito-urinary } \\
\text { system and kidney. }\end{array}$ & General & Pushpangadan and Atal 1984 \\
\hline Aglaia elaeagnoidea (A. Juss.) Benth. & Tree & \begin{tabular}{|l|} 
Leaves, \\
seed
\end{tabular} & Snake and scorpion bites & General & $\begin{array}{l}\text { Ayyanar and Ignacimuthu 2005, } \\
\text { Dey and De } 2012\end{array}$ \\
\hline Aglaia lawii (Wight) C.J. Saldanha & Tree & Bark & Dyspepsia & General & Prasad et al. 2008 \\
\hline Althaea officinalis $\mathrm{L}$. & Herb & Whole plant & Asthma; bronchitis; pneumonia; snake bite & General & $\begin{array}{l}\text { Dey and De 2012, Kayani et al. } \\
2014\end{array}$ \\
\hline Bombax ceiba L. & Tree & $\begin{array}{l}\text { Flower bud, } \\
\text { bark, flower, } \\
\text { fruit, root, }\end{array}$ & $\begin{array}{l}\text { Gynaecological disorders; tumor; regularize menstural } \\
\text { cycle; hydrocele; leucorrhoea; gonorrhoea; sexual }\end{array}$ & $\begin{array}{l}\text { Women, } \\
\text { General }\end{array}$ & $\begin{array}{l}\text { Gairola et al. 2013, Jain et al. } \\
\text { 2004, Namsa et al. } 2009\end{array}$ \\
\hline
\end{tabular}

Published 11 February 2019

http://dx.doi.org/10.32859/era.18.4.1-112 


\begin{tabular}{|c|c|c|c|c|c|}
\hline & & $\begin{array}{l}\text { seed, calyx, } \\
\text { gum, flower }\end{array}$ & $\begin{array}{l}\text { weakness; antidiarrhoeal; abortion; chicken pox; boils; } \\
\text { bums and sores; Diarrhoea }\end{array}$ & & \\
\hline Bombax costatum Pellegr. and Vuillet & Tree & Leaves & Vegetable & General & Dansi et al. 2008 \\
\hline Byttneria andamanensis Kurz & $\begin{array}{l}\text { Climbing } \\
\text { shrub }\end{array}$ & Bark & Hair tonic & General & Prasad et al. 2008 \\
\hline Byttneria herbacea Roxb. & $\begin{array}{l}\text { Climbing } \\
\text { shrub }\end{array}$ & $\begin{array}{l}\text { Leaves, } \\
\text { root, tuber }\end{array}$ & Dysentery; Impaction; wounds; diarrhoea; nerve disorders & General & $\begin{array}{l}\text { Dey et al. 2012, Sreeramulu et } \\
\text { al. } 2013\end{array}$ \\
\hline Cipadessa baccifera (Roth) Miq. & Shrub & $\begin{array}{l}\text { Leaves, } \\
\text { stem, root, } \\
\text { bark }\end{array}$ & $\begin{array}{l}\text { Scorpion; insect and snake bites; diarrhoea; wound; } \\
\text { snakebite; fever; headache; indigestion; infertility; Allegry; } \\
\text { Aphrodisiac; Fever }\end{array}$ & General & $\begin{array}{l}\text { Ayyanar and Ignacimuthu } 2005 \text {, } \\
\text { Kshirsagar and Singh } 2001, \\
\text { Ram et al. 2004, Ramya 2008, } \\
\text { Rao et al. } 2006\end{array}$ \\
\hline Corchorus aestuans L. & Herb & Whole plant & $\begin{array}{l}\text { Anticancer; cardiotonic; chest congestion; obstruction of } \\
\text { abdominal viscera; antipyretic; stomachic; vegetable; } \\
\text { malaria; asthma }\end{array}$ & General & $\begin{array}{l}\text { Dansi et al. 2008, Jayanthi et al } \\
\text { 2012, Pushpangadan and Atal } \\
1984\end{array}$ \\
\hline Corchorus capsularis L. & Herb & Leaves & Chronic cystitis; gonorrhea; dysuria; laxative; health tonic & General & Jayanthi et al. 2012 \\
\hline Corchorus depressus (L.) Stocks & Herb & Whole plant & $\begin{array}{l}\text { pain; fever; sexual dysfunction and to treat gonorrhea; } \\
\text { asthma; cooling medicine; to set-up menstrual disorder } \\
\text { and also as a tonic; to gain sexual vitality }\end{array}$ & $\begin{array}{l}\text { Women; } \\
\text { General }\end{array}$ & Jain et al.2004 \\
\hline Corchorus fascicularis Lam. & Herb & Stem & sex tonic & General & Kosalge and Fursule 2009 \\
\hline Corchorus olitorius L. & Herb & $\begin{array}{l}\text { Seed, } \\
\text { leaves }\end{array}$ & Fever & General & Dansi et al. 2008 \\
\hline Decaschistia crotonifolia Wight and Arn. & Shrub & Fruit & Fracture & General & Rao et al. 2006 \\
\hline $\begin{array}{l}\text { Decaschistia cuddapahensis T.K. Paul } \\
\text { and M.P. Nayar }\end{array}$ & Shrub & - & Fiber yielding plant & General & $\begin{array}{l}\text { Muralidhara Rao and Pullaiah } \\
2007\end{array}$ \\
\hline Eriolaena lushingtonii Dunn & Tree & Whole plant & Antidote for snake bite and scorpion sting & General & $\begin{array}{l}\text { Muralidhara Rao and Pullaiah } \\
2007\end{array}$ \\
\hline Firmiana simplex (L.) W. Wight & Tree & $\begin{array}{l}\text { Gum, wood, } \\
\text { bark }\end{array}$ & $\begin{array}{l}\text { Dysentery; heel cracks; bone fractures; contraceptive; } \\
\text { earache; heart troubles; timber; regularises mensturation; } \\
\text { typhoid }\end{array}$ & $\begin{array}{l}\text { General; } \\
\text { women }\end{array}$ & $\begin{array}{l}\text { Jain et al. 2004, Ratnam and } \\
\text { Raju } 2008\end{array}$ \\
\hline Gossypium herbaceum L. & $\begin{array}{l}\text { Perennial } \\
\text { shrub }\end{array}$ & Root, seed & Contraceptive; oral health care & General & Hebbar et al. 2004 \\
\hline Gossypium hirsutum L. & $\begin{array}{l}\text { Perennial } \\
\text { shrub }\end{array}$ & Leaves & - & General & Xavier et al. 2014 \\
\hline Grewia abutilifolia Vent. ex Juss. & Tree & Fruit, leaves & Wounds and ulcers & Cattle & Rao et al. 2006 \\
\hline Grewia carpinifolia Juss. & Tree & Leaves & Vegetable & General & Dansi et al. 2008 \\
\hline Grewia damine Gaertn. & Tree & Root, leaves & Stomach ulcer; skin disease; boils & General & Kshirsagar and Singh 2001 \\
\hline Grewia flavescens Juss. & Tree & $\begin{array}{l}\text { Bark, wood, } \\
\text { fruit, leaves }\end{array}$ & $\begin{array}{l}\text { Debility; dysentry; opium poisoning; diarrhoea; stomach } \\
\text { pain; skin diseases }\end{array}$ & General & Savithramma et al. 2014 \\
\hline Grewia gamblei J.R. Drumm. & Tree & $\begin{array}{l}\text { Leaves, } \\
\text { bark }\end{array}$ & Snakebite & General & Dey and De 2012 \\
\hline Grewia heterotricha Mast. & Tree & Stem & Cough & General & Sarvalingam et al. 2011 \\
\hline Grewia hirsuta Vahl & Tree & Fruit, bark & Braintonic; antiacidic; dysentery; bronchitis & General & Rao et al. 2006 \\
\hline Grewia lasiodiscus K. Schum. & $\begin{array}{l}\text { Shrub/small } \\
\text { tree }\end{array}$ & Leaves & Vegetable & General & Dansi et al. 2008 \\
\hline
\end{tabular}

Published 11 February 2019

http://dx.doi.org/10.32859/era.18.4.1-112 
Ethnobotany Research and Applications

\begin{tabular}{|c|c|c|c|c|c|}
\hline Grewia mollis Juss. & $\begin{array}{l}\text { Shrub/small } \\
\text { tree }\end{array}$ & Leaves & Vegetable & General & Dansi et al. 2008 \\
\hline Grewia orientalis L. & $\begin{array}{l}\text { Shrub/small } \\
\text { tree }\end{array}$ & Root, leaves & To develop sterility in women; manure; cattle feed & $\begin{array}{l}\text { Women; } \\
\text { cattle }\end{array}$ & Jain et al. 2004 \\
\hline Grewia serrulata DC. & $\begin{array}{l}\text { Shrub/small } \\
\text { tree }\end{array}$ & Fruit & Edible & General & Rekka and Kumar 2014 \\
\hline Grewia tenax (Forssk.) Fiori & $\begin{array}{l}\text { Shrub/small } \\
\text { tree }\end{array}$ & Whole plant & Digestion; reduce body weight & General & Chopda and Mahajan 2009 \\
\hline Grewia tiliifolia Vahl & $\begin{array}{l}\text { Shrub/small } \\
\text { tree }\end{array}$ & $\begin{array}{l}\text { Fruit, bark, } \\
\text { stem, root }\end{array}$ & $\begin{array}{l}\text { Antiasthmatic; digestive disorders; hair fall; diarrhoea; } \\
\text { ulcerative; colitis; expelling placenta }\end{array}$ & $\begin{array}{l}\text { General; } \\
\text { women }\end{array}$ & $\begin{array}{l}\text { Chopda and Mahajan 2009, } \\
\text { Kumar et al. } 2016\end{array}$ \\
\hline Grewia villosa Willd. & $\begin{array}{l}\text { Shrub/small } \\
\text { tree }\end{array}$ & Fruit & Antiinflammatory & General & Kanthasamy Kalaichelvi 2016 \\
\hline Guazuma ulmifolia Lam. & $\begin{array}{l}\text { Shrubby } \\
\text { tree }\end{array}$ & Root & Jaundice & General & Kanthasamy Kalaichelvi 2016 \\
\hline Helicteres isora $\mathrm{L}$. & $\begin{array}{l}\text { Small } \\
\text { tree/large } \\
\text { shrub }\end{array}$ & Whole plant & $\begin{array}{l}\text { Dysentry; diarrhoea; hair growth; snakebite; hair fall; } \\
\text { tympany; insect bite; earache; inflammatory swelling; } \\
\text { intestinal problems; diabetes; cuts; rheumatoid arthritis; }\end{array}$ & General & $\begin{array}{l}\text { Ayyanar and Ignacimuthu 2010, } \\
\text { Padma et al. } 2016\end{array}$ \\
\hline Herissantia crispa (L.) Brizicky & Forb/herb & Fruit & Piles & General & Ignacimuthu et al. 2008 \\
\hline Hibiscus angulosus Steud. ex Mast. & Shrub & Leaves & Cosmetic & General & Das et al. 2013 \\
\hline Hibiscus asper Hook. f. & Shrub & Leaves & Vegetable & General & Dansi et al. 2008 \\
\hline Hibiscus cannabinus L. & Shrub & $\begin{array}{l}\text { Flower } \\
\text { buds, seed, } \\
\text { bark }\end{array}$ & Abortion; non medicinal commercial purposes & General & Ayyanar and Ignacimuthu 2010 \\
\hline Hibiscus hispidissimus Griff. & Shrub & - & Fever & General & Nizar et al. 2015 \\
\hline Hibiscus lobatus (Murray) Kuntze & Shrub & Whole plant & Debility; spermatorrhoea & Men & Malviya et al. 2011 \\
\hline Hibiscus micranthus L.f. & Shrub & $\begin{array}{l}\text { Leaves, } \\
\text { roots, fruit }\end{array}$ & Asthma; diuretic; swelling; edible; urination problems & General & $\begin{array}{l}\text { Kanthasamy Kalaichelvi 2016, } \\
\text { Pradheeps and Poyyamoli } 2013\end{array}$ \\
\hline Hibiscus panduriformis Burm. f. & Shrub & Leaves & Bone fractures & General & Ratnam and Raju 2008 \\
\hline Hibiscus rosa-sinensis L. & Shrub & Whole plant & $\begin{array}{l}\text { Diabetes; menstrual problems; abortion; male reproductive } \\
\text { disorder; jaundice; for good hair; leucorrhea; urinary } \\
\text { disorders; gynaecological disorders; abortifacient; } \\
\text { aphrodisiac; burns; menstural problems; heart problems; } \\
\text { epilepsy; bronchitis; boils; antidiabetic; wound }\end{array}$ & $\begin{array}{l}\text { General; } \\
\text { men; } \\
\text { women }\end{array}$ & Deepak and Gopal 2014 \\
\hline Hibiscus sabdariffa L. & Shrub & $\begin{array}{l}\text { Seed, } \\
\text { flower, } \\
\text { leaves }\end{array}$ & Dysentry; diarrhoea; vegetable & General & Gairola et al. 2013 \\
\hline Hibiscus vitifolius L. & Shrub & Leaves & Diarrhoea & General & Shanmugam 2012 \\
\hline Kavalama urens (Roxb.) Raf. & Tree & Leaves & Menstruation pain & Women & Sreeramulu et al. 2013 \\
\hline Kleinhovia hospita L. & Tree & Leaves & - & General & Chopda and Mahajan 2009 \\
\hline Malva sylvestris $\mathrm{L}$. & Herb & Leaves & Snakebite & General & Dey and De 2012 \\
\hline Malvastrum coromandelianum (L.) Garcke & $\begin{array}{l}\text { Forb/herb/S } \\
\text { ubshrub }\end{array}$ & Leaves & Headache; blisters & General & Ignacimuthu et al. 2008 \\
\hline $\begin{array}{l}\text { Malvaviscus penduliflorus Moc. and Sessé } \\
\text { ex DC. }\end{array}$ & Shrub & Flower & Gynaecological disorders & Women & $\begin{array}{l}\text { Ajesh and Kumuthakalavalli } \\
2012\end{array}$ \\
\hline Melochia corchorifolia L. & Shrub & Leaves, root & Stomachache; vegetable; dysentery & General & Prasad et al. 2008 \\
\hline
\end{tabular}

Published 11 February 2019

http://dx.doi.org/10.32859/era.18.4.1-112 


\begin{tabular}{|c|c|c|c|c|c|}
\hline $\begin{array}{l}\text { Pavonia procumbens (Wight and Arn.) } \\
\text { Walp. }\end{array}$ & Shrub & Whole plant & Body pain; ulcer & General & Ignacimuthu et al. 2008 \\
\hline Pavonia zeylanica (L.) Cav. & Shrub & Root, leaves & $\begin{array}{l}\text { Antipyretic; haemorrhage; dysentery; inflammations; } \\
\text { intestinal problems; eye problems; ulcer }\end{array}$ & General & $\begin{array}{l}\text { Dhivya and Kalaichelvi 2015, } \\
\text { Rao et al. } 2006\end{array}$ \\
\hline Pterocymbium tinctorium Merr. & Tree & Bark & Appetizer & General & Prasad et al. 2008 \\
\hline Pterospermum acerifolium (L.) Willd. & Tree & Bark, flower & Bone fracture & General & Prasad et al. 2008 \\
\hline $\begin{array}{l}\text { Pterospermum rubiginosum B.Heyne ex } \\
\text { Wall. }\end{array}$ & Tree & Bark & Bone fracture; wounds & General & Latheef et al. 2014 \\
\hline Pterospermum xylocarpum (Gaertn.) Oken & Tree & Leaves & Leucorrhoea & General & Savithramma et al. 2014 \\
\hline Pterygota alata (Roxb.) R. Br. & Tree & Bark & Skin diseases & General & Prasad et al. 2008 \\
\hline Sida acuta Burm. f. & $\begin{array}{l}\text { Perennial } \\
\text { herb/shrub }\end{array}$ & Whole plant & $\begin{array}{l}\text { Rheumatism; stomach ache; diuretic; impotency; } \\
\text { insecticidal; wormicidal; skin diseases; snakebite; prevents } \\
\text { infections; vegetable }\end{array}$ & $\begin{array}{l}\text { General; } \\
\text { pregnant } \\
\text { women }\end{array}$ & $\begin{array}{l}\text { Bhardwaj et al. 2011, Chopda } \\
\text { and Mahajan 2009, Dey and De } \\
2012\end{array}$ \\
\hline Sida cordata (Burm. f.) Borss.Waalk. & $\begin{array}{l}\text { Perennial } \\
\text { herb/shrub }\end{array}$ & $\begin{array}{l}\text { Flower, fruit, } \\
\text { leaves, root }\end{array}$ & $\begin{array}{l}\text { Cuts; wounds; diarrhoea; burning sensation in stomach; } \\
\text { rheumatism; boils }\end{array}$ & General & $\begin{array}{l}\text { Dey et al. 2012, Kosalge and } \\
\text { Fursule } 2009\end{array}$ \\
\hline Sida cordifolia L. & $\begin{array}{l}\text { Perennial } \\
\text { herb/shrub }\end{array}$ & Whole plant & $\begin{array}{l}\text { Asthmatic bronchitis; nasel congestion; ulcer; aphrodisiac; } \\
\text { insecticidal; wormicidal; diuretic; dysentery; urinary } \\
\text { trouble; earache; fever; facial paralysis inflammation; ring } \\
\text { worm; rheumatism; nervous problems }\end{array}$ & General & $\begin{array}{l}\text { Chopda and Mahajan 2009, } \\
\text { Dey and De 2012, Suganthi and } \\
\text { Libina } 2015\end{array}$ \\
\hline Sida rhombifolia L. & $\begin{array}{l}\text { Perennial } \\
\text { herb/shrub }\end{array}$ & Whole plant & $\begin{array}{l}\text { Snakebite; skin troubles; diuretic; rheumatism; } \\
\text { tuberculosis; stomachache; wound; headache; eyes } \\
\text { inflammation; debility; arthritis; antibacterial }\end{array}$ & General & $\begin{array}{l}\text { Dansi et al. 2008, Dey and De } \\
\text { 2012, Kumar et al. } 2007\end{array}$ \\
\hline Sida spinosa L. & $\begin{array}{l}\text { Perennial } \\
\text { herb/shrub }\end{array}$ & Root & - & - & Chopda and Mahajan 2009 \\
\hline Sterculia foetida $\mathrm{L}$. & Tree & Fruit & Fumigant & General & Priya and Gopalan 2014 \\
\hline Sterculia guttata Roxb. ex G. Don & Tree & Seed & Edible & General & Bhagat et al. 2016 \\
\hline Sterculia populifolia DC. & Tree & - & Rope making & General & $\begin{array}{l}\text { Muralidhara Rao and Pullaiah } \\
2007\end{array}$ \\
\hline Sterculia setigera Delile & Tree & Leaves & Vegetable & General & Dansi et al. 2008 \\
\hline Sterculia tragacantha Lindl. & Tree & Leaves & Vegetable & General & Dansi et al. 2008 \\
\hline Sterculia villosa Roxb. & Tree & $\begin{array}{l}\text { Root, bark, } \\
\text { gum, seed }\end{array}$ & $\begin{array}{l}\text { Dysentery; facilitates delivery; arthritis; throat problems; } \\
\text { heart problem; asthma; pain; inflammation }\end{array}$ & $\begin{array}{l}\text { General; } \\
\text { pregnant } \\
\text { women }\end{array}$ & Namsa et al. 2009 \\
\hline Theobroma cacao L. & Tree & Fruit & Edible & General & Prabakaran et al. 2013 \\
\hline Thespesia lampas (Cav.) Dalzell & Herb & $\begin{array}{l}\text { Bark, root, } \\
\text { leaves }\end{array}$ & Nervine tonic; antibacterial; jaundice & General & Reddy et al. 2008 \\
\hline Thespesia populnea (L.) Sol. ex Corrêa & Herb & Whole plant & Hepatoprotective; detergent; scabies & General & Ayyanar and Ignacimuthu 2005 \\
\hline Triplochiton scleroxylon K. Schum. & Tree & Leaves & Vegetable & General & Dansi et al. 2008 \\
\hline Triumfetta malebarica J. Koenig ex Rottb. & $\begin{array}{l}\text { Annual herb } \\
\text { to large } \\
\text { shrub }\end{array}$ & Stalk & Impotency & General & Bhogaonkar and Kadam 2006 \\
\hline Triumfetta rhomboidea Jacq. & Shrub & Root, leaves & $\begin{array}{l}\text { Skin eruptions; dysentry; diarrhoea; rheumatism; foot } \\
\text { cracks }\end{array}$ & General & $\begin{array}{l}\text { Kshirsagar and Singh 2001, Rao } \\
\text { et al. } 2006\end{array}$ \\
\hline Triumfetta rotundifolia Lam. & Shrub & Leaves & Migrane & General & Kosalge and Fursule 2009 \\
\hline
\end{tabular}

Published 11 February 2019

http://dx.doi.org/10.32859/era.18.4.1-112 


\begin{tabular}{|c|c|c|c|c|c|}
\hline Urena lobata L. & $\begin{array}{l}\text { Erect, } \\
\text { ascendant } \\
\text { under shrub }\end{array}$ & Whole plant & $\begin{array}{l}\text { Sexual disorder; snakebite; bone fracture; inflammation; } \\
\text { pain releiving; worm infestation; bone strengthening }\end{array}$ & General & $\begin{array}{l}\text { Dey and De 2012, Wagh and } \\
\text { Jain } 2015\end{array}$ \\
\hline Waltheria indica $\mathrm{L}$. & Shrub & Root & Purgative & General & Rao et al. 2006 \\
\hline \multicolumn{6}{|l|}{ Marantaceae } \\
\hline Donax canniformis (G. Forst.) K. Schum. & Shrub & Bark & Fever & General & Prasad et al. 2008 \\
\hline Maranta arundinacea L. & Herb & Leaves, root & Leucorrhoea; antibacterial & General & Valsaraj et al. 1997 \\
\hline \multicolumn{6}{|l|}{ Marattiaceae } \\
\hline Angiopteris evecta (G. Forst.) Hoffm. & Giant fern & Leaves & Hepatitis & General & Udayan et al. 2005 \\
\hline \multicolumn{6}{|l|}{ Marsileaceae } \\
\hline Marsilea minuta L. & Ferns & Whole plant & Anticonvulsive; nervous system & General & $\begin{array}{l}\text { Chakraborty et al. 2012, Sharma } \\
\text { et al. } 2013\end{array}$ \\
\hline Marsilea quadrifolia L. & Ferns & Whole plant & $\begin{array}{l}\text { Fever; diuretic; febrifuge; snakebite; anti-inflammatory; } \\
\text { diuretic; depurative; refrigerant; diabetes; psychiatric } \\
\text { diseases; eye diseases; and diarrhoea and skin disease; } \\
\text { cough; bronchitis; vegetable }\end{array}$ & General & $\begin{array}{l}\text { Ragupathy and Newmaster } \\
\text { 2009, Shanmugam et al. } 2012\end{array}$ \\
\hline \multicolumn{6}{|l|}{ Martyniaceae } \\
\hline Martynia annua L. & Herb & Whole plant & $\begin{array}{l}\text { Boils; blisters; pridontal disorders; epilepsy; sore throat; } \\
\text { alexiteric; inflammations; snakebite; tuberculosis; scabies; } \\
\text { painful urination; gum inflammations }\end{array}$ & General & Jain 2004, Sharma et al. 2013 \\
\hline \multicolumn{6}{|l|}{ Melanthiaceae } \\
\hline Paris polyphylla Sm. & Herb & - & Anti-fungal & General & Zhang et al. 2013 \\
\hline \multicolumn{6}{|l|}{ Melastomataceae } \\
\hline Memecylon umbellatum Burm. f. & Tree & Leaves & Leucorrhoea; diabetes; fish poison; snake bite & General & Kshirsagar and Singh 2001 \\
\hline Melastoma malabathricum L. & Herb & Aerial parts & Cuts; wound; swellings; diarrhoea; dysentery & General & Reddy et al. 2008 \\
\hline $\begin{array}{l}\text { Melastomastrum segregatum (Benth.) A. } \\
\text { Fern. and R. Fern. }\end{array}$ & Shrub & Leaves & Vegetable & General & Dansi et al. 2008 \\
\hline Memecylon gracilis & Herb & Leaves & Fertility in men; affected places of nail & $\begin{array}{l}\text { Men; } \\
\text { general }\end{array}$ & Ayyanar and Ignacimuthu 2005 \\
\hline Memecylon edule Roxb. & Tree & - & Fire wood & General & Prabakaran et al. 2013 \\
\hline Osbeckia zeylanica Steud. ex Naudin & Herb & Whole plant & Disease resistance & General & Ayyanar and Ignacimuthu 2005 \\
\hline Sonerila tinnevelliensis C.E.C. Fisch. & $\begin{array}{l}\text { Erect } \\
\text { subshrub }\end{array}$ & Leaves & Body swelling & General & Rajendran et al. 2000 \\
\hline $\begin{array}{l}\text { Memecylon jadhavii K.N. Reddy, C.S. } \\
\text { Reddy and V.S. Raju }\end{array}$ & Erect Herb & Stem & Fuel & General & Reddy 2006 \\
\hline \multicolumn{6}{|l|}{ Meliaceae } \\
\hline Azadirachta indica A. Juss. & Tree & Whole plant & $\begin{array}{l}\text { Diabetes; malaria; contraceptive; toothache; rheumatism; } \\
\text { chicken pox; wound; cut; jaundice; cough; stomach } \\
\text { worms; leprosy; liver complaints; skin problems; tumors; } \\
\text { spleen; leucoderma; diabetes; fever; dog bite }\end{array}$ & General & $\begin{array}{l}\text { Bhat et al. 2012, Kayani et al. } \\
\text { 2014, Kshirsagar and Singh } \\
\text { 2001, Ragupathy and } \\
\text { Newmaster 2009, Samy et al. } \\
2008\end{array}$ \\
\hline Heynea trijuga Roxb. ex Sims & Tree & $\begin{array}{l}\text { Tender } \\
\text { branches }\end{array}$ & Aphrodisiac & General & Rao et al. 2011 \\
\hline Melia azedarach L. & Tree & $\begin{array}{l}\text { Leaves, } \\
\text { gum, bark, }\end{array}$ & $\begin{array}{l}\text { Small pox; viral fever; skin infections; to control women } \\
\text { hormone problems; antiseptic; antibiotic; stomachache; }\end{array}$ & $\begin{array}{l}\text { General; } \\
\text { women }\end{array}$ & $\begin{array}{l}\text { Dey and De 2012, Khan et al. } \\
2011\end{array}$ \\
\hline
\end{tabular}

Published 11 February 2019

http://dx.doi.org/10.32859/era.18.4.1-112 


\begin{tabular}{|c|c|c|c|c|c|}
\hline & & $\begin{array}{l}\text { stem, root, } \\
\text { flower, seed, } \\
\text { oil, fruit }\end{array}$ & $\begin{array}{l}\text { fertilizer; body pain; heart diseases; elephantisis; asthma; } \\
\text { enlargement; anthelmintic; sores; hysteria }\end{array}$ & & \\
\hline Naregamia alata Wight and Arn. & Shrub & Whole plant & Eczema; pruritus; scabies; jaundice & General & Asha and Pushpangadan 2002 \\
\hline Soymida febrifuga (Roxb.) A. Juss. & Tree & $\begin{array}{l}\text { Bark, } \\
\text { leaves, seed }\end{array}$ & $\begin{array}{l}\text { Reduces pain; regularization mensturation; blood purifier; } \\
\text { gout; shivering; corneal opacity; spleen and liver } \\
\text { enlargement; scorpion sting; malaria }\end{array}$ & $\begin{array}{l}\text { General; } \\
\text { women }\end{array}$ & $\begin{array}{l}\text { Jain et al. 2004, Namsa et al. } \\
2009\end{array}$ \\
\hline Swietenia macrophylla King & Tree & - & $\begin{array}{l}\text { Hypertension; malaria; diabetes; wounds; and skin } \\
\text { ailments }\end{array}$ & General & Eneh et al. 2013 \\
\hline \multicolumn{6}{|l|}{ Menispermaceae } \\
\hline Anamirta cocculus (L.) Wight and Arn. & $\begin{array}{l}\text { Climbing } \\
\text { shrub }\end{array}$ & $\begin{array}{l}\text { Bark, fruit, } \\
\text { leaves, stalk }\end{array}$ & $\begin{array}{l}\text { Convulsive poison and as insecticide; for easy delivery; } \\
\text { fish poison; skin diseases; abortifacient; ear ache; fever; } \\
\text { cough; diarrhoea; Jaundice; vomiting; influenza; lithiasis; } \\
\text { blenorrhagia }\end{array}$ & $\begin{array}{l}\text { General; } \\
\text { pregnant } \\
\text { women }\end{array}$ & $\begin{array}{l}\text { Rahmatullah et al. 2012, } \\
\text { Udayan et al. } 2007\end{array}$ \\
\hline Chasmanthera cordifolia (DC.) Baill. & Climber & Leaves & Diabetes & General & Jaganathan et al. 2016 \\
\hline Cissampelos pareira L. & Shrub & Whole plant & $\begin{array}{l}\text { Headache; fever; burning sensation in chest; Fistula; } \\
\text { Antidode; Blood purification; dysentery; diarrhoea; } \\
\text { snakebite; cough; asthma; liver diseases; fishpoison; } \\
\text { anthelmintic; body pain; antifertility }\end{array}$ & General & $\begin{array}{l}\text { Dey and De 2012, Gairola et al. } \\
2013 \text {, Rao et al. 2006, Valsaraj } \\
\text { et al. } 1997\end{array}$ \\
\hline Cocculus acuminatus DC. & $\begin{array}{l}\text { Climbing } \\
\text { Shrub }\end{array}$ & Stem & Anti-snake venom & General & Sulochana et al. 2015 \\
\hline Cocculus cordifolius DC. & $\begin{array}{l}\text { Climbing } \\
\text { Shrub }\end{array}$ & Leaves & Diabetes & General & Jaganathan et al. 2016 \\
\hline Cocculus hirsutus (L.) W. Theob. & $\begin{array}{l}\text { Climbing } \\
\text { Shrub }\end{array}$ & $\begin{array}{l}\text { Root, } \\
\text { leaves, } \\
\text { rhizome, } \\
\text { stem }\end{array}$ & $\begin{array}{l}\text { Antipyretic and stomachache problems; eczema; } \\
\text { Dysentry; diaherroea; nervine tonic; night blindness; body } \\
\text { cooling; diabetes; epitaxis; urinary problems; laxative; } \\
\text { Sterility development; snake bite; skin infections and } \\
\text { poisonous insects bite }\end{array}$ & General & $\begin{array}{l}\text { Jain et al. 2004, Rao et al. 2006, } \\
\text { Tetali et al. } 2009\end{array}$ \\
\hline Coscinium fenestratum (Goetgh.) Colebr. & $\begin{array}{l}\text { Climbing } \\
\text { Shrub }\end{array}$ & Stem, root & Jaundice; joint pain; headache & General & $\begin{array}{l}\text { Udayan et al. 2005, Xavier et al. } \\
2014\end{array}$ \\
\hline $\begin{array}{l}\text { Cyclea peltata (Lam.) Hook. f. and } \\
\text { Thomson }\end{array}$ & $\begin{array}{l}\text { Climbing } \\
\text { shrub }\end{array}$ & Whole plant & $\begin{array}{l}\text { Fever; stomach pain and bleeding; leucorrhea; snakebite; } \\
\text { boils; blisters; antiulcer; post partum health; antidote; } \\
\text { leprosy; jaundice }\end{array}$ & General & $\begin{array}{l}\text { Abraham and Thomas 2012, } \\
\text { Bosco and Arumugam 2012, } \\
\text { Muralidhara Rao and Pullaiah } \\
2007\end{array}$ \\
\hline Diploclisia glaucescens (Blume) Diels & $\begin{array}{l}\text { Climbing } \\
\text { shrub }\end{array}$ & Root & Herpes; inter trigo; pruritus; scabies & General & Bhat et al. 2014 \\
\hline Menispermum canadense L. & Climber & Root & Skin diseases & General & Suganthi and Libina 2015 \\
\hline Pachygone ovata (Poir.) Diels & $\begin{array}{l}\text { Climbing } \\
\text { shrub }\end{array}$ & $\begin{array}{l}\text { Leaves, } \\
\text { seed }\end{array}$ & Snakebite; to reduce body heat & General & Rajendran et al. 2000 \\
\hline Parabaena sagittata Miers & Climber & Leaves & Incision of snakebite & General & Dey and De 2012 \\
\hline Stephania glandulifera Miers & Climber & Tuber & Rheumatic arthritis; wound healer & General & Namsa et al. 2009 \\
\hline Stephania hernandiifolia (Willd.) Walp. & Climber & Whole plant & - & General & Dey and De 2012 \\
\hline Stephania japonica (Thunb.) Miers & Climber & $\begin{array}{l}\text { Leaves, } \\
\text { root, tuber }\end{array}$ & $\begin{array}{l}\text { Contraceptive; abortion; cuts; wound; scabies; sore; } \\
\text { spermatorrhea; piles; dysentery; ringworm; skin rashes }\end{array}$ & $\begin{array}{l}\text { General; } \\
\text { men; }\end{array}$ & $\begin{array}{l}\text { Kumar et al. 2007, Murty and } \\
\text { Venkaiah } 2010\end{array}$ \\
\hline
\end{tabular}

Published 11 February 2019

http://dx.doi.org/10.32859/era.18.4.1-112 
Ethnobotany Research and Applications

\begin{tabular}{|c|c|c|c|c|c|}
\hline & & & & $\begin{array}{l}\text { pregnant } \\
\text { women }\end{array}$ & \\
\hline Stephania pierrei Diels & Climber & Leaves, root & Blood tonic & General & Srithi et al. 2009 \\
\hline Tiliacora racemosa Colebr. & $\begin{array}{l}\text { Climbing } \\
\text { shrub }\end{array}$ & Leaves, root & Snakebite & General & $\begin{array}{l}\text { Ragupathy and Newmaster } \\
2009\end{array}$ \\
\hline $\begin{array}{l}\text { Tillaea schimperi (Fisch. and C.A. Mey.) } \\
\text { M.G. Gilbert, H. Ohba and K.T. Fu }\end{array}$ & Climber & Leaves & Wounds & General & Puravankara and Gopal 2012 \\
\hline $\begin{array}{l}\text { Tinospora crispa (L.) Hook. f. and } \\
\text { Thomson }\end{array}$ & Climber & Seed & Intoxication due to drugs or alcohol & General & Srithi et al. 2009 \\
\hline Tinospora sinensis (Lour.) Merr. & Climber & $\begin{array}{l}\text { Leaves, } \\
\text { stem, root, } \\
\text { bark }\end{array}$ & $\begin{array}{l}\text { Antiallergic; antibacterial; antidiarrhoeal; aphrodisiac; } \\
\text { asthma; leucorrhea; blood purifier; lactation; menstural } \\
\text { disorder; dysentery; pain; inflammation; insectbite; } \\
\text { jaundice; urinary disorders; joint pain; diabetes; eye sores; } \\
\text { wound; impaction; piles; rheumatism; snakebite; malaria; } \\
\text { typhoid; improves immune system; syphilis }\end{array}$ & $\begin{array}{l}\text { General; } \\
\text { men; } \\
\text { women }\end{array}$ & $\begin{array}{l}\text { Kshirsagar and Singh 2001, } \\
\text { Dey and De 2012, Padma et al. } \\
2016 \text {, Ragupathy and } \\
\text { Newmaster } 2009\end{array}$ \\
\hline \multicolumn{6}{|l|}{ Menyanthaceae } \\
\hline Nymphoides indica (L.) Kuntze & $\begin{array}{l}\text { Perennial, } \\
\text { freshwater } \\
\text { aquatic }\end{array}$ & Leaves, root & Tonsillitis & General & Mukti and Rahmatullah 2013 \\
\hline \multicolumn{6}{|l|}{ Mniaceae } \\
\hline $\begin{array}{l}\text { Plagiomnium cuspidatum (Hedw.) T.J. } \\
\text { Kop. }\end{array}$ & $\begin{array}{l}\text { Exotic auatic } \\
\text { plant }\end{array}$ & - & Used for hemostasis; nose bleeding & General & Chandra et al. 2016 \\
\hline \multicolumn{6}{|l|}{ Molluginaceae } \\
\hline Glinus lotoides L. & Herb & Whole plant & Kidney stones; urinary disorder; insecticidal; wormicidal & General & Bhardwaj et al. 2011 \\
\hline Mollugo cerviana (L.) Ser. & Herb & Whole plant & Amenorrhoea; malaria; abnormal menstruation & $\begin{array}{l}\text { General; } \\
\text { women }\end{array}$ & $\begin{array}{l}\text { Chenniappan and Kadarkarai } \\
2010, \text { Patel } 2010\end{array}$ \\
\hline Mollugo nudicaulis Lam. & Herb & Whole plant & Fever; cough; headache; boils & General & $\begin{array}{l}\text { Ignacimuthu et al. 2008, Kumar } \\
\text { et al. } 2007\end{array}$ \\
\hline Mollugo pentaphylla L. & Herb & Whole plant & $\begin{array}{l}\text { Anti inflammatory; analgesic; antipyretic; antiseptic; sores; } \\
\text { skin diseases; eye diseases }\end{array}$ & General & Ayyanar and Ignacimuthu 2005 \\
\hline \multicolumn{6}{|c|}{ sis } \\
\hline Antiaris toxicaria Lesch. & Tree & Bark & Rheumatic problems; bone fracture & General & Manithottam and Francis 2008 \\
\hline Artocarpus heterophyllus Lam. & Tree & $\begin{array}{l}\text { Root, } \\
\text { leaves, } \\
\text { latex, bark, } \\
\text { fruit, wood }\end{array}$ & $\begin{array}{l}\text { Skin diseases; Ulcer; Asthma; rheumatoid arthritis; } \\
\text { glandular swellings; non medicinal commercial purposes; } \\
\text { antibacterial; Gingival wounds; scabies; ulcer; fever }\end{array}$ & General & $\begin{array}{l}\text { Arjunan et al. 2016, Bhat et al. } \\
\text { 2012, Valsaraj et al. 1997, } \\
\text { Xavier et al. } 2014\end{array}$ \\
\hline Artocarpus hirsutus Lam. & Tree & $\begin{array}{l}\text { Fruit, } \\
\text { leaves, bark, } \\
\text { latex, fruit }\end{array}$ & Snake bite; asthma & General & $\begin{array}{l}\text { Dey and De 2012, Xavier et al. } \\
2014\end{array}$ \\
\hline Brosimum gaudichaudii Trécul & Shrub & - & Mutagenic & General & Rodrigues and Carlini 2005 \\
\hline $\begin{array}{l}\text { Broussonetia papyrifera (L.) L'Hér. ex } \\
\text { Vent. }\end{array}$ & Tree & Bark, fruit & Cough & General & Kayani et al. 2014 \\
\hline Dorstenia brasiliensis Lam. & Herb & Root & Joint pain & General & Hills 2010 \\
\hline Ficus abutilifolia (Miq.) Miq. & Tree & Leaves & Vegetable & General & Dansi et al. 2008 \\
\hline
\end{tabular}

Published 11 February 2019

http://dx.doi.org/10.32859/era.18.4.1-112 


\begin{tabular}{|c|c|c|c|c|c|}
\hline Ficus arnottiana (Miq.) Miq. & Tree & $\begin{array}{l}\text { Leaves, } \\
\text { stem }\end{array}$ & - & - & Chopda and Mahajan 2009 \\
\hline Ficus artocarpoides Warb. & Tree & Leaves & Vegetable & General & Dansi et al. 2008 \\
\hline Ficus asperifolia Miq. & Tree & Leaves & Vegetable & General & Dansi et al. 2008 \\
\hline Ficus benghalensis L. & Tree & $\begin{array}{l}\text { Stem, } \\
\text { leaves, } \\
\text { latex, bark, } \\
\text { root, fruit, } \\
\text { seed }\end{array}$ & $\begin{array}{l}\text { Piles; diabetes; rheumatism; wounds; dental and gum } \\
\text { disorders and toothache; to make semen thick and to } \\
\text { regain sexual potentiality; snakebite; bone fracture; } \\
\text { gonorrhea; psoriasis; mouth ulcer; asthma; flu }\end{array}$ & $\begin{array}{l}\text { General; } \\
\text { men }\end{array}$ & $\begin{array}{l}\text { Ayyanar 2013, Chopda and } \\
\text { Mahajan } 2009\end{array}$ \\
\hline Ficus benjamina $\mathrm{L}$. & Tree & Whole plant & Ulcers; leprosy & General & Kanthasamy Kalaichelvi 2016 \\
\hline Ficus carica L. & Tree & Figs & Warts & General & Shah et al. 2011 \\
\hline Ficus exasperata Vahl & Tree & $\begin{array}{l}\text { Bark, } \\
\text { leaves, root }\end{array}$ & Eczema; pruritus; ringworm; scabies; skin diseases & General & $\begin{array}{l}\text { Bhat et al. 2014, Harsha et al. } \\
2003\end{array}$ \\
\hline Ficus hirta Vahl & Tree & Root, fruit & Wounds caused by snakebites & General & Dey and De 2012 \\
\hline Ficus hispida L.f. & Tree & $\begin{array}{l}\text { Fruit, bark, } \\
\text { latex, } \\
\text { leaves, stem }\end{array}$ & $\begin{array}{l}\text { Boils; uterine infections; tuberculosis; leprosy; blood } \\
\text { purification; induses lactation; aphrodisiac }\end{array}$ & $\begin{array}{l}\text { General; } \\
\text { women }\end{array}$ & $\begin{array}{l}\text { Nizar et al. 2015, Reddy et al. } \\
2008\end{array}$ \\
\hline Ficus infectoria Willd. & Tree & Fruit & Edible & General & Puravankara and Gopal 2012 \\
\hline Ficus ingens (Miq.) Miq. & Tree & Leaves & Vegetable & General & Dansi et al. 2008 \\
\hline Ficus lacor Buch.-Ham. & Tree & Stem & - & General & Chopda and Mahajan 2009 \\
\hline Ficus microcarpa L.f. & Tree & Fruit & Dandruff; wounds & General & Rao et al. 2006 \\
\hline Ficus mollis Vahl & Tree & Fruit, bark & Diabetes; urinary infections & General & Savithramma et al. 2014 \\
\hline Ficus nervosa B. Heyne ex Roth & Tree & Bark & Diabetes & General & Savithramma et al. 2014 \\
\hline Ficus palmata Forssk. & Tree & Fruit & Lung diseases & General & Kayani et al. 2014 \\
\hline Ficus polita Vahl & Tree & Leaves & Vegetable & General & Dansi et al. 2008 \\
\hline $\begin{array}{l}\text { Ficus prostrata (Wall. ex Miq.) Buch. - } \\
\text { Ham. ex Miq. }\end{array}$ & Tree & Root & - & General & Dey and De 2012 \\
\hline Ficus racemosa $\mathrm{L}$. & Tree & $\begin{array}{l}\text { Leaves, } \\
\text { fruit, latex, } \\
\text { root, seed, } \\
\text { bark }\end{array}$ & $\begin{array}{l}\text { Diabetes; eye sight; typhoid; burning micturation; jaundice; } \\
\text { injury; male weakness; diarrhoea; antifertility drug; ulcres; } \\
\text { dysentery; sexual disability }\end{array}$ & $\begin{array}{l}\text { General; } \\
\text { men; } \\
\text { women; } \\
\text { children }\end{array}$ & $\begin{array}{l}\text { Dey and De 2012, Kumar et al. } \\
2007\end{array}$ \\
\hline Ficus religiosa L. & Tree & Whole plant & $\begin{array}{l}\text { Bone fracture; stops bleeding; fever; dullness(children); } \\
\text { obesity decreases; constipation; ear problems; leucorrhea; } \\
\text { alzeimer's disease; glandular disease; dysentery; } \\
\text { menorrhagia; spermatorrhea; swelling; gastrointestinnal } \\
\text { disorders; vomiting; asthma; antibaterial; gonorrhea; } \\
\text { contraceptive }\end{array}$ & $\begin{array}{l}\text { General; } \\
\text { men }\end{array}$ & $\begin{array}{l}\text { Bhat et al. 2014, Kayani et al. } \\
\text { 2014, Rao et al. 2006, Valsaraj } \\
\text { et al. } 1997\end{array}$ \\
\hline Ficus retusa $\mathrm{L}$. & Tree & Fruit, bark & $\begin{array}{l}\text { To cure sterility in men; for body stamina; swelling; } \\
\text { wounds }\end{array}$ & $\begin{array}{l}\text { General; } \\
\text { men }\end{array}$ & Ayyanar and Ignacimuthu 2005 \\
\hline Ficus sur Forssk. & Tree & Leaves & Vegetable & General & Dansi et al. 2008 \\
\hline Ficus sycomorus L. & Tree & Leaves & Vegetable & General & Dansi et al. 2008 \\
\hline Ficus talbotii King & Tree & Bark & Diarrhoea & General & Sreeramulu et al. 2013 \\
\hline $\begin{array}{l}\text { Ficus tinctoria subsp. gibbosa (Blume) } \\
\text { Corner }\end{array}$ & Tree & Stem & External injuries & General & Dey et al. 2012 \\
\hline Ficus variegata Blume & Tree & Fruit & Insecticidal; wormicidal & General & Bhardwaj et al. 2011 \\
\hline
\end{tabular}

Published 11 February 2019

http://dx.doi.org/10.32859/era.18.4.1-112 
Ethnobotany Research and Applications

\begin{tabular}{|c|c|c|c|c|c|}
\hline Ficus virens Aiton & Tree & $\begin{array}{l}\text { Leaves, } \\
\text { bark }\end{array}$ & Skin diseases & General & Sreeramulu et al. 2013 \\
\hline Milicia excelsa (Welw.) C.C. Berg & Tree & Leaves & Vegetable & General & Dansi et al. 2008 \\
\hline Morus alba L. & Tree & $\begin{array}{l}\text { Bark, } \\
\text { leaves, fruit }\end{array}$ & $\begin{array}{l}\text { Cancer; snakebite; purgative; vermifuge; antibacterial; } \\
\text { diaphoretic; hypoglycemic; cuts; wounds }\end{array}$ & General & $\begin{array}{l}\text { Bhagat et al. 2016, Samy et al. } \\
2008\end{array}$ \\
\hline Streblus asper Lour. & Tree & Stem & $\begin{array}{l}\text { Abortifacient; cold; irregular mensturation; anthelmintic; } \\
\text { toothache; oral health care }\end{array}$ & $\begin{array}{l}\text { General; } \\
\text { women; } \\
\text { pregnant } \\
\text { women }\end{array}$ & $\begin{array}{l}\text { Das et al. 2014, Prasad et al. } \\
2008\end{array}$ \\
\hline \multicolumn{6}{|l|}{ Moringaceae } \\
\hline Moringa triloba L. Dun & Tree & Leaves & Fever; cough; cold & General & Kanthasamy Kalaichelvi 2016 \\
\hline Moringa concanensis Nimmo & Tree & Bark, leaves & De-worming; dysentery; fever; cough & General & Sreeramulu et al. 2013 \\
\hline Moringa oleifera Lam. & Tree & Whole plant & $\begin{array}{l}\text { Piles; worm; cough; typhoid; bone fracture; diuretic; } \\
\text { rheumatism; body strengthens; synthesis of sexual } \\
\text { hormones; excretory problems; cuts; wound; snake bite; } \\
\text { dogbite; antibacterial; impotency in male; abdominal pain; } \\
\text { contraceptive; gastrointestinal disorder; anti inflammatory }\end{array}$ & $\begin{array}{l}\text { General; } \\
\text { men }\end{array}$ & $\begin{array}{l}\text { Bhat et al. 2014, Dey and De } \\
\text { 2012, Sharma et al. 2013, } \\
\text { Upadhya et al. } 2012\end{array}$ \\
\hline Moringa pterygosperma Gaertn. & Tree & Bark, leaves & Labour pains; gynaecological disorders & $\begin{array}{l}\text { Women; } \\
\text { pregnant } \\
\text { women }\end{array}$ & Xavier et al. 2014 \\
\hline \multicolumn{6}{|l|}{ Muntingiaceae } \\
\hline Muntingia calabura L. & Tree & Fruit & Edible & General & Bhagat et al. 2016 \\
\hline \multicolumn{6}{|l|}{ Musaceae } \\
\hline Ensete superbum (Roxb.) Cheesman & Erect shrub & $\begin{array}{l}\text { Roots, stem, } \\
\text { seed, bud, } \\
\text { leaves, } \\
\text { tuber, fruit }\end{array}$ & $\begin{array}{l}\text { Bone fracture; kidney stones; urinary infections; } \\
\text { lecucoderma spots; veneral diseases; mad dog bite; to } \\
\text { prevent pregnancy }\end{array}$ & $\begin{array}{l}\text { General; } \\
\text { women }\end{array}$ & $\begin{array}{l}\text { Kosalge and Fursule 2009, } \\
\text { Wagh and Jain } 2015\end{array}$ \\
\hline Musa $\times$ paradisiaca $\mathrm{L}$. & Herb & $\begin{array}{l}\text { Stem, } \\
\text { leaves, bark, } \\
\text { fruit }\end{array}$ & $\begin{array}{l}\text { Antidote; detoxification; snake bite; kidney stone; anti } \\
\text { diabetic; boils; urino genital diseases }\end{array}$ & General & $\begin{array}{l}\text { Bosco and Arumugam 2012, } \\
\text { Samy et al. } 2008\end{array}$ \\
\hline Musa acuminata Colla & Herb & Stem & Kidney stones & General & Jaganathan et al. 2016 \\
\hline Musa balbisiana Colla & Herb & $\begin{array}{l}\text { Root, fruit, } \\
\text { seed, leaves }\end{array}$ & $\begin{array}{l}\text { Dysentery; diarrhoea; bone fracture; contraceptive; } \\
\text { constipation; abortion }\end{array}$ & $\begin{array}{l}\text { General; } \\
\text { pregnant } \\
\text { women }\end{array}$ & Gairola et al. 2013 \\
\hline Musa ornata Roxb. & Herb & Root & Ear ache & General & Rao et al. 2011 \\
\hline \multicolumn{6}{|l|}{ Myricaceae } \\
\hline Myrica nagi Thunb. & Shrub & Stem & - & General & Chopda and Mahajan 2009 \\
\hline \multicolumn{6}{|l|}{ Myristicaceae } \\
\hline $\begin{array}{l}\text { Knema andamanica (Warb.) W.J. de } \\
\text { Wilde }\end{array}$ & Tree & Fruit & Nourishment; fever & General & Chander et al. 2015 \\
\hline Knema globularia (Lam.) Warb. & Tree & Bark & Intestinal affections & General & Prasad et al. 2008 \\
\hline Myristica andamanica Hook. f. & Tree & Nut & Stomachache & General & Prasad et al. 2008 \\
\hline Myristica dactyloides Gaertn. & Tree & Seed & Dysentery & General & Kumar et al. 2007 \\
\hline Myristica fragrans Houtt. & Tree & Leaves & Snake bite; stomach ache & General & Suganthi and Libina 2015 \\
\hline Myristica malabarica Lam. & Tree & Bark & Body pain & General & Binu 2011 \\
\hline
\end{tabular}

Published 11 February 2019

http://dx.doi.org/10.32859/era.18.4.1-112 


\begin{tabular}{|c|c|c|c|c|c|}
\hline \multicolumn{6}{|l|}{ Myrtaceae } \\
\hline Eucalyptus camaldulensis Dehnh. & Tree & Leaves, oil & $\begin{array}{l}\text { Antibacterial; cuts; skin- diseases; sore throat; body pain; } \\
\text { cold; nose infection; flu }\end{array}$ & General & $\begin{array}{l}\text { Jain et al. 2009, Padal et al. } \\
2010\end{array}$ \\
\hline Eucalyptus globulus Labill. & Tree & $\begin{array}{l}\text { Leaves, } \\
\text { stem, seed, } \\
\text { wood, oil }\end{array}$ & $\begin{array}{l}\text { Asthma; cold; cough; throat lozenges; non medicinal } \\
\text { purposes; refreshner; headache; fever; asthma; bronchitis; } \\
\text { migrane; diabetes; antiinflammatory; antiseptic; toothache }\end{array}$ & General & $\begin{array}{l}\text { Mahishi et al. 2005, Manikandan } \\
2005\end{array}$ \\
\hline $\begin{array}{l}\text { Eucalyptus polybractea F. Muell. ex R.T. } \\
\text { Baker }\end{array}$ & Tree & $\begin{array}{l}\text { Leaves, } \\
\text { bark }\end{array}$ & Skin diseases & General & Puravankara and Gopal 2012 \\
\hline Pimenta dioica (L.) Merr. & Tree & Oil & $\begin{array}{l}\text { Anesthetic; analgesic; antimicrobial; antioxidant; } \\
\text { antiseptic; acarcidal; carminative; muscle relaxant; } \\
\text { rubifacient; stimulant and tonic }\end{array}$ & General & Manorama 2015 \\
\hline Psidium guajava L. & Tree & $\begin{array}{l}\text { leaves, fruit, } \\
\text { bark, root, } \\
\text { stem }\end{array}$ & $\begin{array}{l}\text { Cough; antidiarrhoeal; antidysentery; haemorrhages; } \\
\text { headache; mouth freshner; oral health care; toothache; } \\
\text { bronchitis; gastrointestinal disorders; stomach ulcers; } \\
\text { malaria }\end{array}$ & $\begin{array}{l}\text { General; } \\
\text { children }\end{array}$ & $\begin{array}{l}\text { Chopda and Mahajan } 2009 \text {, } \\
\text { Rao et al. } 2006 \text {, Suganthi and } \\
\text { Libina } 2015\end{array}$ \\
\hline Syzygium alternifolium (Wight) Walp. & Tree & Seed, fruit & Diarrhoea; diabetes; wounds & General & Ram et al. 2004 \\
\hline $\begin{array}{l}\text { Syzygium aromaticum (L.) Merr. and L.M. } \\
\text { Perry }\end{array}$ & Tree & $\begin{array}{l}\text { Flower bud, } \\
\text { clove, bark }\end{array}$ & $\begin{array}{l}\text { Indigestion; fever; malaria; headache; diabetes; anorexia; } \\
\text { cough; body ache; nourishment; chicken pox; vomiting; } \\
\text { dandruff; pruritus; scabies; eczema; bed sore; toothache; } \\
\text { skin care; psoriasis }\end{array}$ & General & $\begin{array}{l}\text { Arjunan et al. 2016, Bhat et al. } \\
2014\end{array}$ \\
\hline $\begin{array}{l}\text { Syzygium australe (J.C. Wendl. ex Link) } \\
\text { B. Hyland }\end{array}$ & Tree & Fruit & Antibacterial & General & Sautron and Cock 2014 \\
\hline Syzygium cumini (L.) Skeels & Tree & Whole plant & $\begin{array}{l}\text { Diarrhoea; astringent; menstrual disorders; cold; } \\
\text { bronchitis; asthma; dysentery; blood impurities; ulcers; } \\
\text { indigestion; blood pressure; stomachache; diabetes; } \\
\text { epistaxis; liver disease; snakebite; spleen enlargement; } \\
\text { urine retention; sore throat; mouth ulcer; toothache; } \\
\text { anthelmintic }\end{array}$ & $\begin{array}{l}\text { General; } \\
\text { women }\end{array}$ & $\begin{array}{l}\text { Ayyanar 2013, Bosco and } \\
\text { Arumugam 2012, Chopda and } \\
\text { Mahajan 2009, Dey and De } \\
\text { 2012, Gairola et al. 2013, Jain } \\
\text { 2004, Samy et al. 2008, SM and } \\
\text { Vidyasagar 2013 }\end{array}$ \\
\hline $\begin{array}{l}\text { Syzygium densiflorum Wall. ex Wight and } \\
\text { Arn. }\end{array}$ & Tree & Fruit & Edible & General & Narayan et al. 2011 \\
\hline Syzygium jambos (L.) Alston & Tree & Fruit & Vitamin C source & General & Devi et al. 2010 \\
\hline Syzygium laetum (Buch. -Ham.) Gandhi & Tree & Leaves & Antibacterial & General & Ranjitha et al. 2016 \\
\hline Syzygium rubicundum Wight and Arn. & Tree & Fruit & Antidiarrhoeal; edible & General & Tetali et al. 2009 \\
\hline Syzygium salicifolium (Wight) J. Graham & Tree & Bark, fruit & Diabetes; edible & General & Wagh and Jain 2015 \\
\hline Syzygium zeylanicum (L.) DC. & Tree & Fruit & Strengthens body & General & Duraipandiyan et al. 2006 \\
\hline \multicolumn{6}{|l|}{ Nelumbonaceae } \\
\hline Nelumbo nucifera Gaertn. & $\begin{array}{l}\text { Aquatic } \\
\text { plants }\end{array}$ & Whole plant & $\begin{array}{l}\text { Ringworm; tuberculosis; anti diabetic; boils; bleeding piles; } \\
\text { heart disorders; chronic dysentery }\end{array}$ & General & Mukti and Rahmatullah 2013 \\
\hline \multicolumn{6}{|l|}{ Nepenthaceae } \\
\hline Nepenthes khasiana Hook. f. & Shrub & - & Cataract and night blindness & General & Dhal et al. 2011 \\
\hline \multicolumn{6}{|l|}{ Nephrolepidaceae } \\
\hline Nephrolepis auriculata Trimen & $\begin{array}{l}\text { Epiphytes or } \\
\text { terrestrials } \\
\text { with erect } \\
\text { rhizome }\end{array}$ & Rhizome & Diuretic & General & Rajendran et al. 2000 \\
\hline
\end{tabular}

Published 11 February 2019

http://dx.doi.org/10.32859/era.18.4.1-112 


\begin{tabular}{|c|c|c|c|c|c|}
\hline \multicolumn{6}{|l|}{ Nitrariaceae } \\
\hline Peganum harmala $\mathrm{L}$. & Shrub & Root & Insecticidal; wormicidal & General & Bhardwaj et al. 2011 \\
\hline \multicolumn{6}{|l|}{ Nyctaginaceae } \\
\hline Boerhavia diffusa L. & Herb & Whole plant & $\begin{array}{l}\text { Stomach problems; cough; jaundice and skin diseases; } \\
\text { rheumatism and asthma; boils; blisters; gonorrhea; sores; } \\
\text { swelling; snakebite; Anaemia; asthma; blood purifier; } \\
\text { fever; hastens delivery; inflammation of urinary tract; liver } \\
\text { disease; lactation; epilepsy; wound infections; to promote } \\
\text { pregnancy; cardiac disorders; debility; typhoid }\end{array}$ & $\begin{array}{l}\text { General; } \\
\text { women }\end{array}$ & $\begin{array}{l}\text { Dansi et al. 2008, Prasad et al. } \\
\text { 2008, Ramachandran et al. } \\
\text { 2009, Rao et al. 2006, Sharma } \\
\text { et al. } 2013\end{array}$ \\
\hline Boerhavia erecta L. & Herb & Whole plant & Fever; asthma; vegetable & General & $\begin{array}{l}\text { Dansi et al. 2008, Rao et al. } \\
2006\end{array}$ \\
\hline Boerhavia procumbens Banks ex Roxb. & Herb & Whole plant & Cough and asthma & General & Kayani et al. 2014 \\
\hline Boerhavia repens L. & Herb & Leaves & Anaemia & General & Jagtap et al. 2009 \\
\hline Mirabilis jalapa L. & Herb & Root, leaves & Cuts; wounds; spasmolytic; vegetable & General & Rajan et al. 1997 \\
\hline Pisonia grandis $\mathrm{R}$. Br. & Tree & Leaves & - & General & Xavier et al. 2014 \\
\hline $\begin{array}{l}\text { Pisonia umbellifera (J.R. Forst. and G. } \\
\text { Forst.) Seem. }\end{array}$ & Tree & Bark & Bone fracture; antidiabetic & General & Prasad et al. 2008 \\
\hline \multicolumn{6}{|l|}{ Nymphaeaceae } \\
\hline Euryale ferox Salisb. & $\begin{array}{l}\text { Lily/Aquatic } \\
\text { plant }\end{array}$ & $\begin{array}{l}\text { Thorn, } \\
\text { flower, } \\
\text { leaves }\end{array}$ & Infertility in women & Women & Mukti and Rahmatullah 2013 \\
\hline Nymphaea nouchali Burm. f. & $\begin{array}{l}\text { Aquatic } \\
\text { perennial } \\
\text { herb }\end{array}$ & $\begin{array}{l}\text { Tuber, } \\
\text { leaves } \\
\text { petiole, } \\
\text { flower, root }\end{array}$ & Constipation; hemorrhoids; dysentery; jaundice; dyspepsia & General & Mukti and Rahmatullah 2013 \\
\hline Nymphaea pubescens Willd. & $\begin{array}{l}\text { Aquatic } \\
\text { perennial } \\
\text { herb }\end{array}$ & $\begin{array}{l}\text { Leaves, } \\
\text { flower, root }\end{array}$ & Chronic leucorrhea; black and foul odor of menstrual blood & $\begin{array}{l}\text { General; } \\
\text { women }\end{array}$ & Mukti and Rahmatullah 2013 \\
\hline \multicolumn{6}{|l|}{ Ochnaceae } \\
\hline Ochna obtusata DC. & Shrub & $\begin{array}{l}\text { Root, bark, } \\
\text { leaves }\end{array}$ & Bone fracture; head-ache; skin diseases; snakebite & General & $\begin{array}{l}\text { Rao et al. 2006, Sreeramulu et } \\
\text { al. } 2013\end{array}$ \\
\hline \multicolumn{6}{|l|}{ Olacaceae } \\
\hline Olax scandens Roxb. & Tree & $\begin{array}{l}\text { Root, flower, } \\
\text { bark }\end{array}$ & Stomachache; diarrhoea; fever; cough & General & Sreeramulu et al. 2013 \\
\hline Ximenia americana L. & Shrub & $\begin{array}{l}\text { Bark, fruit, } \\
\text { root }\end{array}$ & Rheumatism; dogbite; oral health care & General & Hebbar et al. 2004 \\
\hline \multicolumn{6}{|l|}{ Oleaceae } \\
\hline Chionanthus ramiflorus Roxb. & $\begin{array}{l}\text { Shrub and } \\
\text { tree }\end{array}$ & Fruit & Sores & General & Rao et al. 2006 \\
\hline Chionanthus zeylanicus L. & $\begin{array}{l}\text { Shrub and } \\
\text { tree }\end{array}$ & $\begin{array}{l}\text { Leaves, } \\
\text { seed }\end{array}$ & Worm infestation & General & Rao et al. 2006 \\
\hline Jasminum angustifolium (L.) Willd. & $\begin{array}{l}\text { Climbing } \\
\text { shrub }\end{array}$ & Leaves & Skin diseases; ulcer; anti-toxic & General & Kanthasamy Kalaichelvi 2016 \\
\hline Jasminum arborescens Roxb. & Shrub & Leaves & Mammary glands secretion of milk stops & Women & Jain et al. 2004 \\
\hline
\end{tabular}

Published 11 February 2019

http://dx.doi.org/10.32859/era.18.4.1-112 


\begin{tabular}{|c|c|c|c|c|c|}
\hline Jasminum auriculatum Vahl & Shrub & $\begin{array}{l}\text { Leaves, } \\
\text { bark, stem, } \\
\text { root, flower }\end{array}$ & Tympany; Boils; Blisters; Wounds; Cough; bone fractures & General & $\begin{array}{l}\text { Chopda and Mahajan } 2009 \text {, } \\
\text { Sreeramulu et al. } 2013\end{array}$ \\
\hline Jasminum cuspidatum Rottl. and Willd. & Shrub & $\begin{array}{l}\text { Flowers, } \\
\text { leaves }\end{array}$ & Anti-septic; antispasmodic; fevers; urinary inflammation & General & Kanthasamy Kalaichelvi 2016 \\
\hline Jasminum grandiflorum $\mathrm{L}$. & Shrub & Whole plant & $\begin{array}{l}\text { Liver disease; dysentery; mental alertness treatment; } \\
\text { tumor; headache; boils; eczema }\end{array}$ & General & $\begin{array}{l}\text { Bhat et al. 2014, Chopda and } \\
\text { Mahajan } 2009\end{array}$ \\
\hline Jasminum humile L. & Shrub & Leaves & Sinusitis & General & Kayani et al. 2014 \\
\hline Jasminum malabaricum Wight & Shrub & Sap, leaves & Cataract; conjuctivitis; diabetes & General & Lingaraju et al. 2013 \\
\hline Jasminum officinale L. & Shrub & Leaves, root & Mouth ulcers & General & Chopda and Mahajan 2009 \\
\hline Jasminum sambac (L.) Aiton & Shrub & $\begin{array}{l}\text { Latex, stem, } \\
\text { leaves, seed }\end{array}$ & Diarrhoea & General & Chopda and Mahajan 2009 \\
\hline Ligustrum lucidum W.T. Aiton & $\begin{array}{l}\text { Shrub or } \\
\text { small tree }\end{array}$ & - & Anti-microbial & General & Zhang et al. 2013 \\
\hline Ligustrum vulgare L. & $\begin{array}{l}\text { Shrub or } \\
\text { small tree }\end{array}$ & $\begin{array}{l}\text { Leaves, } \\
\text { bark }\end{array}$ & Vulnerary antiseptic & General & Kanthasamy Kalaichelvi 2016 \\
\hline Nyctanthes arbor-tristis $\mathrm{L}$. & Shrub & Leaves, root & $\begin{array}{l}\text { Malaria; fits; bone fracture; cuts; wound; snakebite; rough } \\
\text { skin; urinary disorders }\end{array}$ & General & Jain 2004, Rao et al. 2006 \\
\hline Olea dioica Roxb. & Tree & Fruit & $\begin{array}{l}\text { Edible; hepatoprotection; anti-inflammation; } \\
\text { immunomodulation; anticarcinogenicity; anti-infertility }\end{array}$ & General & Bhagat et al. 2016 \\
\hline Olea europaea L. & Tree & Leaves & Fever; cough; cold; lowers pressure & General & Parada et al. 2009 \\
\hline Schrebera swietenioides Roxb. & Tree & Fruit & Eye diseases & General & Wagh and Jain 2015 \\
\hline \multicolumn{6}{|l|}{ Onagraceae } \\
\hline Ludwigia adscendens (L.) H. Hara & $\begin{array}{l}\text { Perennial } \\
\text { herb }\end{array}$ & Whole plant & Cattle wounds & General & Dey and De 2012 \\
\hline Ludwigia decurrens Walter & $\begin{array}{l}\text { Perennial } \\
\text { herb }\end{array}$ & Leaves & Vegetable & General & Dansi et al. 2008 \\
\hline Ludwigia octovalvis (Jacq.) P.H. Raven & $\begin{array}{l}\text { Perennial } \\
\text { herb }\end{array}$ & Whole plant & For venereal diseases & General & Manikandan 2005 \\
\hline Ludwigia perennis L. & $\begin{array}{l}\text { Perennial } \\
\text { herb }\end{array}$ & Leaves & Menstural disorder & General & Chander et al. 2015 \\
\hline Ludwigia peruviana (L.) H. Hara & $\begin{array}{l}\text { Perennial } \\
\text { herb }\end{array}$ & Whole plant & Dysentery; Purgative; vermifuge & General & Kanthasamy Kalaichelvi 2016 \\
\hline \multicolumn{6}{|l|}{ Ophioglossaceae } \\
\hline Helminthostachys zeylanica (L.) Hook. & Ferns & - & Hepatoprotective; Aphrodisiac & General & Rajasekharan and Latha \\
\hline \multicolumn{6}{|l|}{ Opiliaceae } \\
\hline Cansjera rheedii & Erect shrub & Leaves, fruit & Diabetes; edible & General & Savithramma et al. 2014 \\
\hline \multicolumn{6}{|l|}{ Orchidaceae } \\
\hline Aerides crispum Lindl. & Orchid & Whole plant & Ear deafness & General & Rajendran et al. 1997 \\
\hline Bulbophyllum fischeri Seidenf. & Orchid & Whole plant & Antibacterial activity & General & Ranjitha et al.2016 \\
\hline Coelogyne nervosa A. Rich. & Orchid & Whole plant & Antibacterial activity & General & Ranjitha et al. 2016 \\
\hline Crepidium acuminatum (D. Don) Szlach. & Orchid & Stem, bulb & Stomach pain; tuberculosis & General & Das et al. 2013 \\
\hline Cymbidium aloifolium (L.) Sw. & Orchid & Root, leaves & Ear drops to relief pain; bone fracture; foot cracks & General & $\begin{array}{l}\text { Rajendran et al. 1997, } \\
\text { Ragupathy and Newmaster } \\
2009\end{array}$ \\
\hline
\end{tabular}

Published 11 February 2019

http://dx.doi.org/10.32859/era.18.4.1-112 


\begin{tabular}{|c|c|c|c|c|c|}
\hline Dactylorhiza hatagirea (D. Don) Soó & Orchid & Tuber & Cuts and wounds & General & Kumar et al. 2007 \\
\hline Dendrobium herbaceum Lindl. & Orchid & Whole plant & Antibacterial activity; ear ache & General & Ranjitha et al. 2016 \\
\hline Dendrobium macrostachyum Lindl. & Orchid & Whole plant & Leg pains & General & Prasad et al. 2008 \\
\hline Dendrobium microbulbon A.Rich. & Orchid & Bulb & Edible & General & Gavali and Sharma 2004 \\
\hline Disa ovalifolia Sond. & Orchid & Wood & Firewood & General & Jayakumar et al. 2010 \\
\hline Eulophia dabia (D. Don) Hochr. & Orchid & Bulb & Impotency & General & Bhogaonkar and Kadam 2006 \\
\hline $\begin{array}{l}\text { Eulophia epidendraea (J. Koenig ex Retz.) } \\
\text { C.E.C. Fisch. }\end{array}$ & Orchid & $\begin{array}{l}\text { Rhizome, } \\
\text { tuber }\end{array}$ & Anorexia; boils & General & Rajendran et al. 1997 \\
\hline Eulophia spectabilis (Dennst.) Suresh & Orchid & Tuber, root & Snake bite; rheumatoid arthritis & General & Dey and De 2012 \\
\hline Habenaria andamanica Hook. f. & Orchid & Tuber & Venereal diseases & General & Prasad et al. 2008 \\
\hline $\begin{array}{l}\text { Habenaria commelinifolia (Roxb.) Wall. ex } \\
\text { Lindl. }\end{array}$ & Orchid & Bulb & Vegetable & General & Wagh and Jain 2015 \\
\hline Habenaria digitata Lindl. & Orchid & Bulb & Wound and cuts & General & Wagh and Jain 2015 \\
\hline Habenaria furcifera Lindl. & Orchid & Bulb & Leucorrhoea & General & Wagh and Jain 2015 \\
\hline Habenaria marginata Colebr. & Orchid & Bulb, tuber & Jaundice; tonic & General & Wagh and Jain 2015 \\
\hline Habenaria plantaginea Lindl. & Orchid & Tuber & Chest pain and stomach pain & General & Rajendran et al. 1997 \\
\hline Habenaria roxburghii Nicolson & Orchid & $\begin{array}{l}\text { Leaves, } \\
\text { tuber, root }\end{array}$ & $\begin{array}{l}\text { Tonic; snakebite; insect bite; cooling effect; dysentery; } \\
\text { breast cancer }\end{array}$ & General & Rajendran et al. 1997 \\
\hline Luisia macrantha Blatt. and McCann & Orchid & Whole plant & Antibacterial activity & General & Ranjitha et al. 2016 \\
\hline Malaxis rheedii B. Heyne ex Wallace & Orchid & Tuber & Insect bite & General & Reddy et al. 2005 \\
\hline $\begin{array}{l}\text { Mycaranthes pannea (Lindl.) S.C. Chen } \\
\text { and J.J. Wood }\end{array}$ & Orchid & Leaves & $\begin{array}{l}\text { Dislocated portion of joint to relieve severe pain; swelling; } \\
\text { dislocation and fracture }\end{array}$ & General & Namsa et al. 2009 \\
\hline Nervilia concolor (Blume) Schltr. & Orchid & Whole plant & Kidney stones; wound; lactation; menstural cycle problems & $\begin{array}{l}\text { General; } \\
\text { women }\end{array}$ & Udayan et al. 2005 \\
\hline Nervilia infundibulifolia Blatt. and McCann & Orchid & - & Epilepsy & General & Chithra et al. 2016 \\
\hline Oberonia brunoniana Wight & Orchid & Whole plant & Antibacterial activity & General & Ranjitha et al. 2016 \\
\hline Pecteilis susannae (L.) Raf. & Orchid & Tuber & Snake bite & General & Lingaraju et al. 2013 \\
\hline Pholidota imbricata Lindl. & Orchid & Whole plant & Antibacterial & General & Ranjitha et al. 2016 \\
\hline Pholidota pallida Lindl. & Orchid & Rhizome & Finger absus & General & Rajendran et al. 1997 \\
\hline Phyllorchis nilgherensis (Wight) Kuntze & Orchid & Whole plant & For scabies; leucoderma; antibacterial & General & Udayan et al. 2005 \\
\hline Pinalia mysorensis (Lindl.) Kuntze & Orchid & Whole plant & Antibacterial activity & General & Ranjitha et al. 2016 \\
\hline Pleione bulbocodioides (Franch.) Rolfe & Orchid & - & Anti-bacterial & General & Zhang et al. 2013 \\
\hline Rhynchostylis retusa (L.) Blume & Orchid & Leaves, fruit & Ear sore & General & Bhat et al. 2014 \\
\hline Vanda spathulata (L.) Spreng. & Orchid & Flower & Antiasthmatic & General & Kumar et al. 2016 \\
\hline Vanda tessellata (Roxb.) Hook. ex G. Don & Orchid & Whole plant & $\begin{array}{l}\text { Malaria; fever; scorpion bite; bone fracture; snakebite; } \\
\text { antibacterial }\end{array}$ & $\begin{array}{l}\text { General; } \\
\text { children }\end{array}$ & Rajendran et al. 1997 \\
\hline Vanda testacea (Lindl.) Rchb. f. & Orchid & Whole plant & Bone fracture & General & Rao et al. 2011 \\
\hline Zeuxine longilabris (Lindl.) Trimen & Orchid & Whole plant & Cough & General & Rajendran et al. 1997 \\
\hline \multicolumn{6}{|l|}{ Orobanchaceae } \\
\hline Striga angustifolia (D. Don) C.J. Saldanha & $\begin{array}{l}\text { Erect } \\
\text { scabrid herb }\end{array}$ & Whole plant & Contraceptive & Women & Bhogaonkar and Kadam 2006 \\
\hline Striga asiatica (L.) Kuntze & $\begin{array}{l}\text { Erect } \\
\text { scabrid herb }\end{array}$ & Fruit & Urinary disorders & General & Rao et al. 2006 \\
\hline
\end{tabular}

Published 11 February 2019

http://dx.doi.org/10.32859/era.18.4.1-112 
Ethnobotany Research and Applications

\begin{tabular}{|c|c|c|c|c|c|}
\hline Striga gesnerioides (Willd.) Vatke & $\begin{array}{l}\text { Erect } \\
\text { scabrid herb }\end{array}$ & Whole plant & Antibacterial activity & General & Ranjitha et al. 2016 \\
\hline \multicolumn{6}{|l|}{ Oxalidaceae } \\
\hline Averrhoa carambola L. & Tree & Fruit, bark & Vitamin C source; liver disease & General & Jain 2004 \\
\hline Biophytum reinwardtii (Zucc.) Klotzsch & Tree & Whole plant & Dysentery; scabies & General & Udayan et al. 2005 \\
\hline Biophytum sensitivum (L.) DC. & Tree & Whole plant & $\begin{array}{l}\text { Asthma; chest complaints; convulsions; diabetes; } \\
\text { inflammatory; stomachache; tumors; fever; snake bite; } \\
\text { boils; blisters; cuts; poisonous insect bite; leech bite; } \\
\text { hydrocele; body pain; Epilepsy; diabetes; veneral diseases }\end{array}$ & General & $\begin{array}{l}\text { Ayyanar 2013, Chopda and } \\
\text { Mahajan 2009, Dey and De } \\
\text { 2012, Ignacimuthu 2005, } \\
\text { Pushpangadan and Atal 1984, } \\
\text { Xavier et al. } 2014 \\
\end{array}$ \\
\hline Oxalis corniculata L. & $\begin{array}{l}\text { Perennial } \\
\text { herb }\end{array}$ & Whole plant & $\begin{array}{l}\text { Epilepsy; diarrhoea; dyspepsia; hemorrhoids; piles; } \\
\text { burning sensation; anaemia; gas trouble; insecticidal; } \\
\text { wormicidal; snakebite; syphilis; dysentery; stomach pain; } \\
\text { reduces body heat; antibacterial }\end{array}$ & General & $\begin{array}{l}\text { Bhardwaj et al. 2011, Rao et al. } \\
2006\end{array}$ \\
\hline Oxalis debilis Kunth & $\begin{array}{l}\text { Perennial } \\
\text { herb }\end{array}$ & Whole plant & - & - & Dey and De 2012 \\
\hline \multicolumn{6}{|l|}{ Paeoniaceae } \\
\hline Paeonia $\times$ suffruticosa Andrews & Herb & - & Anti-microbial & General & Zhang et al. 2013 \\
\hline Paeonia emodi Royle & Herb & Leaves, root & - & General & Sharma et al. 2013 \\
\hline Paeonia lactiflora Pall. & Herb & - & Anti-inflammatory & General & Zhang et al. 2013 \\
\hline \multicolumn{6}{|l|}{ Pandanaceae } \\
\hline Pandanus amaryllifolius Roxb. & $\begin{array}{l}\text { Erect, stout- } \\
\text { boled small } \\
\text { tree }\end{array}$ & $\begin{array}{l}\text { Aerial parts, } \\
\text { oil }\end{array}$ & - & General & Sharma et al. 2013 \\
\hline Pandanus dubius Spreng. & $\begin{array}{l}\text { Erect, stout- } \\
\text { boled small } \\
\text { tree }\end{array}$ & Root & Diarrhoea & General & Prasad et al. 2008 \\
\hline Pandanus odorifer (Forssk.) Kuntze & $\begin{array}{l}\text { Erect, stout- } \\
\text { boled small } \\
\text { tree }\end{array}$ & $\begin{array}{l}\text { Root, fruit, } \\
\text { leaves }\end{array}$ & Vegetable; fibre; non medicinal commercial purposes & General & $\begin{array}{l}\text { Ragupathy and Newmaster } \\
2009\end{array}$ \\
\hline Pandanus thwaitesii Martelli & $\begin{array}{l}\text { Erect, stout- } \\
\text { boled small } \\
\text { tree }\end{array}$ & $\begin{array}{l}\text { Apical } \\
\text { meristem }\end{array}$ & Jaundice & General & Rajendran et al. 2000 \\
\hline \multicolumn{6}{|l|}{ Papaveraceae } \\
\hline Argemone aenea Ownbey & Forb/herb & Whole plant & $\begin{array}{l}\text { Skin infection; ulcers; cracked foot; skin rashes and } \\
\text { disease; eye infection; jaundice; scabies; ophthalmia; } \\
\text { dysentery; leucoderma; Gonerrhea; conjuctivitis; liver } \\
\text { disease; liquid film in eye; rheumatism; eczema; piles; } \\
\text { headache; stomach complaints }\end{array}$ & General & $\begin{array}{l}\text { Alagesaboopathi 2012, Chopda } \\
\text { and Mahajan 2009, } \\
\text { Subramanian et al. } 2011\end{array}$ \\
\hline Argemone mexicana $\mathrm{L}$. & Forb/herb & Seed, root & Malaria; oral health care & General & Hebbar et al. 2004 \\
\hline $\begin{array}{l}\text { Corydalis yanhusuo (Y.H. Chou and Chun } \\
\text { C. Hsu) W.T. Wang ex Z.Y. Su and C.Y. } \\
\text { Wu }\end{array}$ & Herb & - & Anti-cancer & General & Zhang et al. 2013 \\
\hline Fumaria parviflora Lam. & Herb & Aerial parts & Insecticidal; wormicidal & General & Bhardwaj et al. 2011 \\
\hline Papaver somniferum L. & Herb & Seed & - & - & Chopda and Mahajan 2009 \\
\hline
\end{tabular}

Published 11 February 2019

http://dx.doi.org/10.32859/era.18.4.1-112 


\begin{tabular}{|c|c|c|c|c|c|}
\hline \multicolumn{6}{|l|}{ Passifloraceae } \\
\hline Adenia hondala (Gaertn.) W.J. de Wilde & Herb & $\begin{array}{l}\text { Leaves, } \\
\text { tuber, root }\end{array}$ & $\begin{array}{l}\text { Joint pain; Antiseptic; larvicidal; as body stimulant and for } \\
\text { lactation. }\end{array}$ & $\begin{array}{l}\text { women; } \\
\text { General }\end{array}$ & $\begin{array}{l}\text { Das et al. 2013, Lingaraju et al. } \\
2013\end{array}$ \\
\hline Decaloba leschenaultii (DC.) M. Roem. & $\begin{array}{l}\text { Slender } \\
\text { climbers }\end{array}$ & Leaves & Dysentery & General & Ignacimuthu et al. 2008 \\
\hline Passiflora calcarata Mast. & Herb & Whole plant & Skin diseases & General & Kennedy 2006 \\
\hline Passiflora edulis Sims & Herb & Leaves, fruit & Vitamin C source; swellings & General & Rekka and Kumar 2014 \\
\hline Passiflora foetida L. & Herb & Whole plant & Headache; arthritic problems; jaundice; asthma; vegetable & General & $\begin{array}{l}\text { Dansi et al. 2008, Rao et al. } \\
2006, \text { Sharma et al. } 2013\end{array}$ \\
\hline Passiflora subpeltata Ortega & Herb & Leaves, fruit & Jaundice & General & Lingaraju et al. 2013 \\
\hline \multicolumn{6}{|l|}{ Pedaliaceae } \\
\hline Ceratotheca sesamoides Endl. & Herb & Leaves & Vegetable & General & Dansi et al. 2008 \\
\hline Pedalium murex $\mathrm{L}$. & Herb & $\begin{array}{l}\text { Leaves, } \\
\text { root, fruit }\end{array}$ & $\begin{array}{l}\text { Fever(cow); diuretic; gonorrhea; for vigour in men; } \\
\text { diabetes; fertility in women; toothache; cold; cough; } \\
\text { diarrhoea }\end{array}$ & $\begin{array}{l}\text { General; } \\
\text { women; } \\
\text { men; cow }\end{array}$ & $\begin{array}{l}\text { Jain et al. 2004, Rao et al. 2006, } \\
\text { Shanmugam et al. } 2012\end{array}$ \\
\hline Sesamum alatum Thonn. & $\begin{array}{l}\text { Erect annual } \\
\text { herb }\end{array}$ & Whole plant & Boils; blisters; cuts & General & Sreeramulu et al. 2013 \\
\hline Sesamum indicum L. & $\begin{array}{l}\text { Erect annual } \\
\text { herb }\end{array}$ & $\begin{array}{l}\text { Leaves, } \\
\text { seed, oil, } \\
\text { flower, gum }\end{array}$ & $\begin{array}{l}\text { Diuretic; night blindness; abdominal pain; increases } \\
\text { lactation; reduces inflammation; rheumatism; dandruff }\end{array}$ & $\begin{array}{l}\text { General; } \\
\text { women }\end{array}$ & Bhat et al. 2014 \\
\hline $\begin{array}{l}\text { Sesamum radiatum Schumach. and } \\
\text { Thonn. }\end{array}$ & $\begin{array}{l}\text { Erect annual } \\
\text { herb }\end{array}$ & Leaves & Vegetable & General & Dansi et al. 2008 \\
\hline \multicolumn{6}{|l|}{ Pentaphylacaceae } \\
\hline Eurya japonica Thunb. & Tree & - & $\begin{array}{l}\text { Prepare a soft beverage with Cinnamomum zeylanicum } \\
\text { and fruits of Elettaria cardamomum }\end{array}$ & General & Pushpangadan and Atal 1984 \\
\hline \multicolumn{6}{|l|}{ Phrymaceae } \\
\hline Mazus pumilus (Burm. f.) Steenis & Forb/herb & Leaves & - & General & Sharma et al. 2013 \\
\hline \multicolumn{6}{|l|}{ Phyllanthaceae } \\
\hline Antidesma acidum Retz. & $\begin{array}{l}\text { Shrub or } \\
\text { small tree }\end{array}$ & Leaves & Riped are for eating; Sour unripe fruits are pickled & General & Horo and Topno 2015 \\
\hline Antidesma alexiteria $\mathrm{L}$. & $\begin{array}{l}\text { Shrub or } \\
\text { small tree }\end{array}$ & Leaves & Antidysenteric; Sunstroke; proper digestion & General & Dey and De 2012 \\
\hline Antidesma ghaesembilla Gaertn. & $\begin{array}{l}\text { Shrub or } \\
\text { small tree }\end{array}$ & Fruit & To treat malaria and malaria-like symptoms & General & Nazarudeen 2010 \\
\hline Baccaurea courtallensis (Wight) Müll. Arg. & Tree & Fruit & $\begin{array}{l}\text { The aril is sweetish sour and the rind is pickled.eat the ripe } \\
\text { fruits }\end{array}$ & General & Nazarudeen 2010 \\
\hline Bischofia javanica Blume & Tree & Bark & Skin diseases & General & Prasad et al. 2008 \\
\hline Breynia retusa (Dennst.) Alston & Tree & Leaves, root & Cough; antiasthmatic & General & Kumar et al. 2016 \\
\hline Breynia rhamnoides Müll. Arg. & $\begin{array}{l}\text { Perennial } \\
\text { tree }\end{array}$ & $\begin{array}{l}\text { Bark, stem } \\
\text { sap, leaves, } \\
\text { root }\end{array}$ & $\begin{array}{l}\text { White patches on the skin all over the body; cataract; eye } \\
\text { inflammation; memory power }\end{array}$ & General & Mahishi et al. 2005 \\
\hline Breynia vitis-idaea (Burm. f.) C.E.C. Fisch. & Tree & Leaves & Psoriasis; Scabies; indigestion & General & Prasad et al. 2013 \\
\hline Bridelia montana (Roxb.) Willd. & Tree & $\begin{array}{l}\text { Leaves, } \\
\text { bark, root }\end{array}$ & Boils; Blisters; Cuts & General & Padal et al. 2014 \\
\hline
\end{tabular}

Published 11 February 2019

http://dx.doi.org/10.32859/era.18.4.1-112 
Ethnobotany Research and Applications

\begin{tabular}{|c|c|c|c|c|c|}
\hline Bridelia retusa (L.) A. Juss. & $\begin{array}{l}\text { Large shrub } \\
\text { or tree }\end{array}$ & - & Fractured bones & General & Krishna et al. 2011 \\
\hline Bridelia stipularis (L.) Blume & Tree & $\begin{array}{l}\text { Stem, bark, } \\
\text { leaves, root }\end{array}$ & $\begin{array}{l}\text { Wounds healing; to remove wound scars; abdominal pain; } \\
\text { scabies; eczema; wound; bone fracture }\end{array}$ & General & $\begin{array}{l}\text { Udayan et al. 2005, Upadhya et } \\
\text { al. } 2012\end{array}$ \\
\hline $\begin{array}{l}\text { Cleistanthus collinus (Roxb.) Benth. ex } \\
\text { Hook. f. }\end{array}$ & Shrub & $\begin{array}{l}\text { Bark, } \\
\text { leaves, stem }\end{array}$ & $\begin{array}{l}\text { Skin boils; Blisters; Wounds; Antidote against snake bite; } \\
\text { Insecticide; fish poison; skin problem; wounds; tooth ache; } \\
\text { fertilizer }\end{array}$ & General & $\begin{array}{l}\text { Dubey et al. 2012, Kumar and } \\
\text { Chaturvedi 2010, Rao et al. } \\
2006\end{array}$ \\
\hline Flueggea leucopyrus Willd. & Shrub & Leaves, fruit & Boils; eczema; edible; sores & General & Bhat et al. 2014 \\
\hline $\begin{array}{l}\text { Glochidion heyneanum (Wight and Arn.) } \\
\text { Wight }\end{array}$ & $\begin{array}{l}\text { Small tree or } \\
\text { large shrub }\end{array}$ & Fruit, stem & Diabetes; fever; bone fracture & General & Savithramma et al. 2014 \\
\hline Glochidion zeylanicum (Gaertn.) A. Juss. & $\begin{array}{l}\text { Small tree or } \\
\text { large shrub }\end{array}$ & Bark, leaves & Snake bite; stomach ulcers & General & Das et al. 2013 \\
\hline Phyllanthus acidus (L.) Skeels & Tree & Fruit & $\begin{array}{l}\text { Remove dandruff; cure night blindness; antivomiting; } \\
\text { diabetes }\end{array}$ & General & Kumar and Chaturvedi 2010 \\
\hline $\begin{array}{l}\text { Phyllanthus amarus Schumach. and } \\
\text { Thonn. }\end{array}$ & Tree & Whole plant & $\begin{array}{l}\text { Abortion; jaundice; dysentery; diabetes; diarrhoea; } \\
\text { hepatitis; malaria; vegetable; wound }\end{array}$ & $\begin{array}{l}\text { General; } \\
\text { pregnant } \\
\text { women }\end{array}$ & Ayyanar 2013 \\
\hline Phyllanthus debilis Klein ex Willd. & Tree & Leaves & Jaundice & General & Pradheeps and Poyyamoli 2013 \\
\hline Phyllanthus emblica L. & Tree & Whole plant & $\begin{array}{l}\text { Anorexia; impaction; antibacterial; asthma; aphrodisiac; } \\
\text { jaundice; improves eyesight; bronchitis; mouth ulcer; blood } \\
\text { pressure; constipation; indigestion; snakebite; skin } \\
\text { disease; toothache; stomach disorder; diabetes; } \\
\text { inflammation; postpartum health; contraceptive; anaemia; } \\
\text { menorrhegia }\end{array}$ & General & $\begin{array}{l}\text { Ayyanar } 2013 \text {, Jain } 2004 \text {, } \\
\text { Prabakaran et al. 2013, Udayan } \\
\text { et al. } 2007\end{array}$ \\
\hline Phyllanthus fraternus G.L. Webster & Tree & Whole plant & Jaundice; gonorrhoea; tuberculosis; spermaturia & $\begin{array}{l}\text { General; } \\
\text { men }\end{array}$ & Dhanabal et al. 2015 \\
\hline Phyllanthus indofischeri Bennet & Tree & Fruit & Good tonic; diuretic & General & Kanthasamy Kalaichelvi 2016 \\
\hline Phyllanthus lawii J. Graham & Tree & Whole plant & Toothache and throat pain & General & Pushpangadan and Atal 1984 \\
\hline Phyllanthus niruri L. & Tree & Whole plant & $\begin{array}{l}\text { Bronchitis; leprosy; anemia; urinary discharge; anuria; } \\
\text { biliousness; asthma; jaundice; genitourinary system } \\
\text { problems; snakebite }\end{array}$ & General & $\begin{array}{l}\text { Lingaraju et al. 2013, Samy et } \\
\text { al. } 2008\end{array}$ \\
\hline Phyllanthus reticulatus Poir. & Tree & Whole plant & $\begin{array}{l}\text { Stimulant; astringent; anthelmatic; diarrhoea; diuretic; } \\
\text { snake bite; antimicrobial; skin diseases; bone fracture; } \\
\text { insect bite; psoriasis }\end{array}$ & General & $\begin{array}{l}\text { Ignacimuthu et al. 2008, Kumar } \\
\text { and Chaturvedi } 2010\end{array}$ \\
\hline Phyllanthus rheedei Wight & Tree & Whole plant & Liver diseases; jaundice & General & Suresh and Asha 2008 \\
\hline Phyllanthus virgatus G. Forst. & Tree & Leaves & Reduce excessive body heat; stomachache; jaundice & General & Ayyanar and Ignacimuthu 2005 \\
\hline \multicolumn{6}{|l|}{ Pinaceae } \\
\hline Abies pindrow (Royle ex D. Don) Royle & Tree & Leaves & Cough; phthisis; asthma; chronic & General & Kayani et al. 2014 \\
\hline Pinus gerardiana Wall. ex D. Don & Tree & Seed & & General & Chopda and Mahajan 2009 \\
\hline Pinus palustris Mill. & Tree & Leaves, oil & Ritual & General & Chopda and Mahajan 2009 \\
\hline Pinus wallichiana A.B. Jacks. & Tree & $\begin{array}{l}\text { Resin, root, } \\
\text { leaves }\end{array}$ & Resin; rituals; wound; fracture; tuberculosis & General & Ruba and Mohan 2016 \\
\hline \multicolumn{6}{|l|}{ Piperaceae } \\
\hline Peperomia pellucida (L.) Kunth & Herb & Fruit & Fever & General & Chander et al. 2015 \\
\hline
\end{tabular}

Published 11 February 2019

http://dx.doi.org/10.32859/era.18.4.1-112 
Ethnobotany Research and Applications

\begin{tabular}{|c|c|c|c|c|c|}
\hline $\begin{array}{l}\text { Peperomia tetraphylla (G. Forst.) Hook. } \\
\text { and Arn. }\end{array}$ & Herb & Whole plant & Sores on scalp; mouth sores; nasal blisters & General & $\begin{array}{l}\text { Rajendran et al. 2000, Rao et al. } \\
2011\end{array}$ \\
\hline Peperomia wightiana Wight & Herb & Leaves & Indigestion & Children & Kumar et al. 2007 \\
\hline Piper attenuatum Buch. -Ham. ex Miq. & $\begin{array}{l}\text { Climbing } \\
\text { shrub }\end{array}$ & $\begin{array}{l}\text { Fruit, stem, } \\
\text { root, wood }\end{array}$ & $\begin{array}{l}\text { Throat pain; cough; chest pain; fever cough; asthma or } \\
\text { other pulmonary infections. }\end{array}$ & General & Rao et al. 2006 \\
\hline Piper betle L. & $\begin{array}{l}\text { Climbing } \\
\text { shrub }\end{array}$ & $\begin{array}{l}\text { Nut, leaves, } \\
\text { petiole, root }\end{array}$ & $\begin{array}{l}\text { Abortifacient; bad breath; fermentation; constipation; } \\
\text { contraceptive; cuts; burns; diabetes; intestinal } \\
\text { disorders(cattle); wound; malaria; chicken pox; to prevent } \\
\text { thrist; gum bleeding; vomiting; indigestion; skin diseases }\end{array}$ & $\begin{array}{l}\text { General; } \\
\text { cattle; } \\
\text { pregnant } \\
\text { women; } \\
\text { children; } \\
\text { women }\end{array}$ & $\begin{array}{l}\text { Latheef et al. 2014, Nizar et al. } \\
\text { 2015, Ruba and Mohan } 2016\end{array}$ \\
\hline Piper cubeba Bojer & $\begin{array}{l}\text { Climbing } \\
\text { shrub }\end{array}$ & Leaves, root & Inflammatory joints; genito-urinary infections & $\begin{array}{l}\text { Women; } \\
\text { general }\end{array}$ & Pushpangadan and Atal 1984 \\
\hline Piper hymenophyllum (Miq.) Wight & $\begin{array}{l}\text { Climbing } \\
\text { shrub }\end{array}$ & Leaves & Mouth ulcers & General & Bhakshu et al. 2016 \\
\hline Piper longum L. & $\begin{array}{l}\text { Climbing } \\
\text { shrub }\end{array}$ & $\begin{array}{l}\text { Fruit, root, } \\
\text { spike, } \\
\text { leaves, } \\
\text { flower, seed }\end{array}$ & $\begin{array}{l}\text { Bronchitis; toothache; anaemia; antibacterial; } \\
\text { contraceptive; cough; fever; cold; respiratory infections; } \\
\text { tumors; asthma; headache }\end{array}$ & General & $\begin{array}{l}\text { Nizar et al. 2015, Udayan et al. } \\
2007\end{array}$ \\
\hline Piper mullesua Buch. -Ham. ex D. Don & $\begin{array}{l}\begin{array}{l}\text { Climbing } \\
\text { shrub }\end{array} \\
\end{array}$ & Seed & - & General & Kennedy 2006 \\
\hline Piper nigrum L. & $\begin{array}{l}\text { Climbing } \\
\text { shrub }\end{array}$ & $\begin{array}{l}\text { Seed, } \\
\text { leaves, fruit, } \\
\text { flower, root }\end{array}$ & $\begin{array}{l}\text { Antibacterial; cough; bronchial disorders; snake bite; } \\
\text { dandruff; scabies; eczema; bed sore; boils; throat } \\
\text { infection; malaria; tooth decay; cold; cough; dysentery; } \\
\text { fever; stomach problems }\end{array}$ & General & $\begin{array}{l}\text { Lingaraju et al. 2013, Sharma et } \\
\text { al. } 2013\end{array}$ \\
\hline Piper retrofractum Vahl & $\begin{array}{l}\text { Climbing } \\
\text { shrub }\end{array}$ & Leaves & Asthma & General & Rajagopal et al. 2016 \\
\hline Piper trioicum Roxb. & $\begin{array}{l}\text { Climbing } \\
\text { shrub }\end{array}$ & $\begin{array}{l}\text { Fruit, stem, } \\
\text { root }\end{array}$ & $\begin{array}{l}\text { Cough and chest pain; fever cough; asthma or other } \\
\text { pulmonary infections. }\end{array}$ & General & Pushpangadan and Atal 1984 \\
\hline Piper tuberculatum Jacq. & $\begin{array}{l}\text { Climbing } \\
\text { shrub }\end{array}$ & - & Hypotensive & General & Rodrigues and Carlini 2015 \\
\hline Piper wallichii (Miq.) Hand. -Mazz. & $\begin{array}{l}\text { Climbing } \\
\text { shrub }\end{array}$ & Fruit & - & General & Chopda and Mahajan 2009 \\
\hline \multicolumn{6}{|l|}{ Pittosporaceae } \\
\hline Pittosporum neelgherrense Wight and Arn. & $\begin{array}{l}\text { Tree, shrub } \\
\text { or } \\
\text { undershrub }\end{array}$ & Bark & Hepatoprotective; snake bite; liver problems & General & Prasad et al. 2013 \\
\hline Pittosporum tetraspermum Wight and Arn. & $\begin{array}{l}\text { Tree, shrub } \\
\text { or } \\
\text { undershrub }\end{array}$ & Stem, bark & Snakebite & General & Udayan et al. 2005 \\
\hline Pittosporum wightii A.K. Mukh. & $\begin{array}{l}\text { Tree, shrub } \\
\text { or } \\
\text { undershrub }\end{array}$ & Fruit & Sexual disorder & General & Wagh and Jain 2015 \\
\hline
\end{tabular}




\begin{tabular}{|c|c|c|c|c|c|}
\hline Bacopa monnieri (L.) Wettst. & Herb & Whole plant & $\begin{array}{l}\text { Snakebite; To prevent hair loss; brain tonic; nervous } \\
\text { disorder; epilepsy; Stammering; Anaemia; Polyuria; } \\
\text { Rejuvenator; memory capacity; free flow of urine; } \\
\text { constipation; stomach disorders; malaria; promotes } \\
\text { fertility; psycological disorder }\end{array}$ & $\begin{array}{l}\text { General; } \\
\text { children }\end{array}$ & $\begin{array}{l}\text { Dey and De 2012, Prasad et al. } \\
2008, \text { Rao et al. 2016, Sharma } \\
\text { et al. } 2013\end{array}$ \\
\hline Limnophila heterophylla (Roxb.) Benth. & Herb & Whole plant & Leprosy; dysentery; wound & General & Manikandan 2005 \\
\hline Plantago asiatica $\mathrm{L}$. & Herb & - & Anti-inflammatory & General & Zhang et al. 2013 \\
\hline Plantago lanceolata L. & Herb & $\begin{array}{l}\text { Leaves, } \\
\text { seed, fruit }\end{array}$ & Cough; whooping cough; bronchitis; boils & General & Kayani et al. 2014 \\
\hline Plantago major L. & Herb & Whole plant & $\begin{array}{l}\text { Stomach ache; stomach inflammation; wound healing; and } \\
\text { burns }\end{array}$ & $\begin{array}{l}\text { General; } \\
\text { veterinary }\end{array}$ & Ruba and Mohan 2016 \\
\hline Plantago ovata Forssk. & Herb & Seed, fruit & Cold; cough; abortion & General & $\begin{array}{l}\text { Pushpakarani and Natarajan } \\
2014\end{array}$ \\
\hline Rehmannia glutinosa (Gaertn.) DC. & Herb & - & Anti-tumour & General & Zhang et al. 2013 \\
\hline Scoparia dulcis $\mathrm{L}$. & Herb & Whole plant & $\begin{array}{l}\text { Diabetes; jaundice; antiseptic; hypertension; sickle cell } \\
\text { disease; fever; leucorrhea; kidney stones; increases } \\
\text { lactation; stomach problems; cuts; urinary tract infections; } \\
\text { respiratory disorders; menstural problems; malaria; } \\
\text { sexually transmitted diseases; cancer; dysentery; } \\
\text { diabetes; seminal disease }\end{array}$ & $\begin{array}{l}\text { General; } \\
\text { women; } \\
\text { men }\end{array}$ & $\begin{array}{l}\text { Ignacimuthu et al. 2008, } \\
\text { Lingaraju et al. 2013, Sayed et } \\
\text { al. 2007, Suganthi and Libina } \\
\text { 2015, Udayan et al. 2005, } \\
\text { Xavier et al. } 2014\end{array}$ \\
\hline Veronica beccabunga L. & Herb & Whole plant & - & General & Chopda and Mahajan 2009 \\
\hline \multicolumn{6}{|l|}{ Plumbaginaceae } \\
\hline Plumbago auriculata Lam. & Shrub & Root & Acidity & General & Sharma et al. 2013 \\
\hline Plumbago indica L. & Shrub & $\begin{array}{l}\text { Tuber, } \\
\text { leaves, root }\end{array}$ & Rheumatism; abortion; antibacterial; leprosy; eczema & $\begin{array}{l}\text { General; } \\
\text { pregnant } \\
\text { women }\end{array}$ & Murty and Venkaiah 2010 \\
\hline Plumbago zeylanica L. & Shrub & Whole plant & $\begin{array}{l}\text { Abortifacient; sexual; debility; antifertility; asthma; breast } \\
\text { cancer; fits; eczema; skin diseases; rheumatism; tympany; } \\
\text { jaundice; leucoderma; gastritis; typhoid; arithritis; } \\
\text { ringworm; gynaecological disorders; scorpion stings; sore } \\
\text { wounds; snakebite; stomachache; diuretic; diarrhoea; } \\
\text { pyrexia; }\end{array}$ & $\begin{array}{l}\text { General; } \\
\text { pregnant } \\
\text { women; } \\
\text { women }\end{array}$ & $\begin{array}{l}\text { Latheef et al. 2014, Murty and } \\
\text { Venkaiah 2010, Padma et al. } \\
\text { 2016, Ramya } 2008\end{array}$ \\
\hline \multicolumn{6}{|c|}{ ( } \\
\hline Andropogon abyssinicus R. Br. ex Fresen. & Grass & Root & To digest food and to increase secretion of milk & $\begin{array}{l}\text { Women; } \\
\text { General }\end{array}$ & Chopda and Mahajan 2009 \\
\hline Aristida setacea Retz. & Grass & Whole plant & As a thatching material & General & Dileep and Nair 2015 \\
\hline Arundinella setosa Trin. & Grass & Whole plant & Fodder; lactation & $\begin{array}{l}\text { Cattle; } \\
\text { General }\end{array}$ & Jayanthi et al. 2012 \\
\hline Axonopus compressus (Sw.) P. Beauv. & Grass & Whole plant & $\begin{array}{l}\text { As a permanent cover and turf to moist; low fertility soil; } \\
\text { particularly in shaded situations; to prevent soil erosions }\end{array}$ & General & Dileep and Nair 2015 \\
\hline Bambusa bambos (L.) Voss & Grass & Whole plant & $\begin{array}{l}\text { Post labor pains; nervous disorder; rheumatism; cuts; } \\
\text { tuberculosis; wasting diseases; non medicinal purposes; } \\
\text { abnormal mensturation; bone setting; anaemia; to clear } \\
\text { uterus after child birth; Wound healing; abortifacient; skin } \\
\text { disorders; obesity; ulcers }\end{array}$ & $\begin{array}{l}\text { General; } \\
\text { pregnant } \\
\text { women; } \\
\text { women }\end{array}$ & $\begin{array}{l}\text { Ayyanar 2013, Latheef et al. } \\
2014, \text { Ragupathy and } \\
\text { Newmaster } 2009\end{array}$ \\
\hline
\end{tabular}

Published 11 February 2019

http://dx.doi.org/10.32859/era.18.4.1-112 


\begin{tabular}{|c|c|c|c|c|c|}
\hline Bothriochloa pertusa (L.) A. Camus & Grass & Whole plant & For treating neuralgia; headache & General & Dileep and Nair 2015 \\
\hline Brachiaria ramosa (L.) Stapf & Grass & Whole plant & Used as animal fodder for cattle; goat; sheep and poultry & Animals & Dileep and Nair 2015 \\
\hline Cenchrus ciliaris L. & Grass & Root, leaves & $\begin{array}{l}\text { Kidney pain; Wound healing; tumors; insecticidal; } \\
\text { wormicidal }\end{array}$ & General & Bhardwaj et al. 2011 \\
\hline Cenchrus setiger Vahl & Grass & Root & Insecticidal; wormicidal & General & Bhardwaj et al. 2011 \\
\hline Chloris barbata Sw. & Grass & Leaves & Skin diseases; fever; diarrhea; Diabetes & General & Kanthasamy Kalaichelvi 2016 \\
\hline Chrysopogon aciculatus (Retz.) Trin. & Grass & $\begin{array}{l}\text { Rhizome, } \\
\text { root }\end{array}$ & $\begin{array}{l}\text { For stomach ache and gastric disorders; diarrhea and } \\
\text { dysentery; jaundice }\end{array}$ & General & Chander et al. 2015 \\
\hline Chrysopogon zizanioides (L.) Roberty & Grass & Whole plant & $\begin{array}{l}\text { Burns; epilepsy; snake bite; scorpion sting; mouth sores; } \\
\text { tooth ache; urinary tract infection; malaria; fever; sprains; } \\
\text { rheumatism }\end{array}$ & General & $\begin{array}{l}\text { Sharma et al. 2013, Sulochana } \\
\text { et al. } 2015\end{array}$ \\
\hline Coix lacryma-jobi L. & Grass & Whole plant & $\begin{array}{l}\text { For treating abdominal tumours;fever; diabetes; dysentery; } \\
\text { gastro intestinal and lung diseases; retarding aging; } \\
\text { poultry feed; treating skin diseases such as acne and } \\
\text { other swellings; post partum health; urinary disorders; } \\
\text { Uterine diseases; menstrual problems }\end{array}$ & $\begin{array}{l}\text { General; } \\
\text { women }\end{array}$ & $\begin{array}{l}\text { Chithra et al. 2016, Dileep and } \\
\text { Nair } 2015\end{array}$ \\
\hline Cymbopogon citratus (DC.) Stapf & Grass & Whole plant & $\begin{array}{l}\text { Muscular rheumatism; nasal bleeding; antibacterial; snake } \\
\text { bite; pimples }\end{array}$ & General & $\begin{array}{l}\text { Bosco and Arumugam 2012, } \\
\text { Sharma et al. } 2013\end{array}$ \\
\hline $\begin{array}{l}\text { Cymbopogon flexuosus (Nees ex Steud.) } \\
\text { W. Watson }\end{array}$ & Grass & Whole plant & $\begin{array}{l}\text { Headache; pesticide; cholera; flavouring and perfumery } \\
\text { agent; nebulisation }\end{array}$ & General & Manikandan 2005 \\
\hline Cymbopogon giganteus Chiov. & Grass & Leaves & Vegetable & General & Dansi et al. 2008 \\
\hline Cymbopogon jwarancusa (Jones) Schult. & Grass & Leaves & Insecticidal; wormicidal & General & Bhardwaj et al. 2011 \\
\hline Cymbopogon martini (Roxb.) W. Watson & Grass & Whole plant & Typhoid; on grey hair for blackening & General & Rajendran et al. 2002 \\
\hline Cymbopogon nardus (L.) Rendle & Grass & Stem & Bronchitis; rheumatism & General & $\begin{array}{l}\text { Prashanth Kumar and } \\
\text { Shiddamallayya } 2015\end{array}$ \\
\hline Cynodon dactylon (L.) Pers. & Grass & Whole plant & $\begin{array}{l}\text { Diabetes; blood pressure; skin problems; blood purifier; } \\
\text { wounds; dysentery; diarrhoea; snakebite; diuretic; syphilis; } \\
\text { urinary tract infections; toothache; vomiting; excessive } \\
\text { menstural discharge; dysuria; contraceptive; rheumatism; } \\
\text { asthma; headache; stomach problems; indigestion }\end{array}$ & General & $\begin{array}{l}\text { Dey and De 2012, Kanthasamy } \\
\text { Kalaichelvi 2016, Pradheeps } \\
\text { and Poyyamoli } 2013\end{array}$ \\
\hline Dactyloctenium aegyptium (L.) Willd. & Grass & Whole plant & $\begin{array}{l}\text { Dysentery; anti-inflammatory; worm infection; wounds; } \\
\text { kidney pain; ulcer }\end{array}$ & General & Dileep and Nair 2015 \\
\hline Dendrocalamus strictus (Roxb.) Nees & Grass & Leaves & $\begin{array}{l}\text { Abortion; dysentery; cough; oedema; indigestion; wound; } \\
\text { ulcers }\end{array}$ & $\begin{array}{l}\text { General; } \\
\text { women; } \\
\text { cow(dysente } \\
\text { ry) }\end{array}$ & $\begin{array}{l}\text { Padal et al. 2014, Rao et al. } \\
2006\end{array}$ \\
\hline Desmostachya bipinnata (L.) Stapf & Grass & Leaves & $\begin{array}{l}\text { Dysentery; Menorhhagia; diuretic; in geriatric care; } \\
\text { asthma; tuberculosis }\end{array}$ & $\begin{array}{l}\text { General; } \\
\text { women }\end{array}$ & $\begin{array}{l}\text { Dileep and Nair 2015, Kayani et } \\
\text { al. } 2014\end{array}$ \\
\hline Echinochloa colona (L.) Link & Grass & Whole plant & Edible & General & Dileep and Nair 2015 \\
\hline Eleusine coracana (L.) Gaertn. & Grass & Fruit, leaves & $\begin{array}{l}\text { Dysentery; for wound healing; measles; pleurisy and } \\
\text { smallpox fevers; ulcer; diabetes }\end{array}$ & General & $\begin{array}{l}\text { Latheef et al. 2014, Sreeramulu } \\
\text { et al. } 2013\end{array}$ \\
\hline Eleusine indica (L.) Gaertn. & Grass & Whole plant & $\begin{array}{l}\text { Snakebite; fever; for reducing blood sugar level; } \\
\text { dysentery; prevent soil erosion; antiabortion }\end{array}$ & $\begin{array}{l}\text { General; } \\
\text { pregnant } \\
\text { women }\end{array}$ & $\begin{array}{l}\text { Dey and De 2012, Srithi et al. } \\
2009\end{array}$ \\
\hline
\end{tabular}

Published 11 February 2019

http://dx.doi.org/10.32859/era.18.4.1-112 
Ethnobotany Research and Applications

\begin{tabular}{|c|c|c|c|c|c|}
\hline $\begin{array}{l}\text { Eragrostis unioloides (Retz.) Nees ex } \\
\text { Steud. }\end{array}$ & Grass & Whole plant & Asthma; wound & General & Dileep and Nair 2015 \\
\hline Hackelochloa granularis (L.) Kuntze & Grass & Whole plant & Bruise & General & Kshirsagar and Singh 2001 \\
\hline $\begin{array}{l}\text { Heteropogon contortus (L.) P. Beauv. ex } \\
\text { Roem. and Schult. }\end{array}$ & Grass & Whole plant & Used as a stimulant and diuretic; toothache; snake bite & General & Dileep and Nair 2015 \\
\hline Imperata cylindrica (L.) Raeusch. & Grass & Bark & Muscle pains & General & Rao et al. 2006 \\
\hline Isachne globosa (Thunb.) Kuntze & Grass & Whole plant & Sinusitis & General & Dileep and Nair 2015 \\
\hline $\begin{array}{l}\text { Ochlandra scriptoria (Dennst.) C.E.C. } \\
\text { Fisch. }\end{array}$ & Grass & Stem & Wounds & General & Udayan et al. 2005 \\
\hline Ochlandra travancorica (Bedd.) Gamble & Grass & Clums & Gynaecological disorders & Women & $\begin{array}{l}\text { Ajesh and Kumuthakalavalli } \\
2012\end{array}$ \\
\hline Oplismenus compositus (L.) P. Beauv. & Grass & Whole plant & Inscision of snakebite & General & Dey and De 2012 \\
\hline Oryza rufipogon Griff. & Grass & Seed & Diuretic; abdominal pain & General & $\begin{array}{l}\text { Prashanth Kumar and } \\
\text { Shiddamallayya } 2015\end{array}$ \\
\hline Oryza sativa L. & Grass & Husk, seed & Tooth cleaning & General & Deepa et al. 2011 \\
\hline Panicum maximum Jacq. & Grass & Whole plant & Antimalarial and analgesic activity & General & Suganthi and Libina 2015 \\
\hline Panicum repens $\mathrm{L}$. & Grass & Rhizome & Stomach ache and gastric disorders & General & $\begin{array}{l}\text { Dileep and Nair 2015, Hebbar et } \\
\text { al. } 2004\end{array}$ \\
\hline Panicum sumatrense Roth & Grass & - & To treat the shivers & General & Maloles et al. 2011 \\
\hline Paspalidium flavidum (Retz.) A. Camus & Grass & Seed & Substitute for rice & General & Dileep and Nair 2015 \\
\hline Paspalum scrobiculatum L. & Grass & $\begin{array}{l}\text { Stem, } \\
\text { rhizome, } \\
\text { root, seed }\end{array}$ & $\begin{array}{l}\text { Diuretic; diabetes; wound healing; bowel cleanser; } \\
\text { reduces inflammation; vitamin supplement }\end{array}$ & $\begin{array}{l}\text { General; } \\
\text { children }\end{array}$ & $\begin{array}{l}\text { Dileep and Nair 2015, Maloles et } \\
\text { al. } 2011\end{array}$ \\
\hline $\begin{array}{l}\text { Pennisetum hohenackeri Hochst. ex } \\
\text { Steud. }\end{array}$ & Grass & Whole plant & Hair growth; thatching material & General & Dileep and Nair 2015 \\
\hline Pennisetum purpureum Schumach. & Grass & $\begin{array}{l}\text { Stem, } \\
\text { leaves }\end{array}$ & Abnormal blood flow after menstruation & Women & Nanjunda 2010 \\
\hline Phragmites australis (Cav.) Trin. ex Steud. & Grass & Root & - & General & Chopda and Mahajan 2009 \\
\hline Saccharum arundinaceum Retz. & Grass & Root & Kidney stone & General & Dileep and Nair 2015 \\
\hline Saccharum bengalense Retz. & Grass & Leaves & Menstrual problem; remedy for abortion & $\begin{array}{l}\text { Women; } \\
\text { pregnant } \\
\text { women }\end{array}$ & Jain et al. 2004 \\
\hline Saccharum officinarum L. & Grass & Stem & Jaundice; laxative & $\begin{array}{l}\text { General; } \\
\text { women }\end{array}$ & Ruba and Mohan 2016 \\
\hline Saccharum spontaneum L. & Grass & Whole plant & $\begin{array}{l}\text { Aphrodisiac; galactogogue; mental illness; fever; fodder for } \\
\text { goats; blood related disorders; dyspepsia; burning } \\
\text { sensation; piles ; respiratory and gynaecological troubles }\end{array}$ & $\begin{array}{l}\text { General; } \\
\text { women }\end{array}$ & $\begin{array}{l}\text { Dileep and Nair 2015, Sharma } \\
\text { et al. } 2013\end{array}$ \\
\hline Sacciolepis interrupta (Willd.) Stapf & Grass & Root, seed & Respiratory disorders; grains are edible & General & Dileep and Nair 2015 \\
\hline Setaria italica (L.) P. Beauv. & Grass & Whole plant & $\begin{array}{l}\text { Shivering; diuretic; dyspepsia; poor digestion; bone } \\
\text { fracture }\end{array}$ & General & Maloles et al. 2011 \\
\hline Setaria viridis (L.) P. Beauv. & Grass & - & Shivering & General & Maloles et al. 2011 \\
\hline Sorghum bicolor (L.) Moench & Grass & - & Measles; pleurisy and smallpox fevers & General & Maloles et al. 2011 \\
\hline Sporobolus wallichii Munro ex Thwaites & Grass & Whole plant & To enrich the blood & General & Dileep and Nair 2015 \\
\hline Themeda cymbaria Hack. & Grass & - & Thatching & General & Prabakaran et al. 2013 \\
\hline Themeda triandra Forssk. & Grass & Whole plant & Wounds & General & Ayyanar and Ignacimuthu 2005 \\
\hline
\end{tabular}

Published 11 February 2019

http://dx.doi.org/10.32859/era.18.4.1-112 
Ethnobotany Research and Applications

\begin{tabular}{|c|c|c|c|c|c|}
\hline $\begin{array}{l}\text { Thysanolaena latifolia (Roxb. ex Hornem.) } \\
\text { Honda }\end{array}$ & Grass & Whole plant & Piles & General & Ruba and Mohan 2016 \\
\hline Triticum aestivum $\mathrm{L}$. & Grass & Fruit, seed & Eczema; boils & General & Bhat et al. 2014 \\
\hline \multicolumn{6}{|l|}{ Podocarpaceae } \\
\hline Podocarpus neriifolius D. Don & Tree & Leaves & Rheumatism & General & Prasad et al. 2008 \\
\hline \multicolumn{6}{|l|}{ Polygalaceae } \\
\hline Polygala arvensis Willd. & $\begin{array}{l}\text { Perennial } \\
\text { herb or } \\
\text { shrub }\end{array}$ & Whole plant & Snake bite & General & Suthari and Raju 2016 \\
\hline Polygala chinensis $\mathrm{L}$. & $\begin{array}{l}\text { Perennial } \\
\text { herb or } \\
\text { shrub }\end{array}$ & Leaves, root & Asthma; chronic bronchitis and catarrhal affection; fever & General & Thirunarayanan 2013 \\
\hline Polygala elongata Klein ex Willd. & $\begin{array}{l}\text { Perennial } \\
\text { herb or } \\
\text { shrub }\end{array}$ & Root, bark & Headache & General & Thirunarayanan 2013 \\
\hline Polygala senega L. & $\begin{array}{l}\text { Perennial } \\
\text { herb or } \\
\text { shrub }\end{array}$ & Leaves, root & - & General & Thirunarayanan 2013 \\
\hline Polygala tenuifolia Willd. & $\begin{array}{l}\text { Perennial } \\
\text { herb or } \\
\text { shrub }\end{array}$ & - & Anti-microbial & General & Zhang et al. 2013 \\
\hline Securidaca Iongipedunculata Fresen. & Tree & Leaves & Vegetable & General & Dansi et al. 2008 \\
\hline \multicolumn{6}{|l|}{ Polygonaceae } \\
\hline Calligonum polygonoides $\mathrm{L}$. & Shrub & Whole plant & Typhoid & General & Porte 2014 \\
\hline $\begin{array}{l}\text { Persicaria amplexicaulis (D. Don) Ronse } \\
\text { Decr. }\end{array}$ & Shrub & $\begin{array}{l}\text { Root, } \\
\text { leaves, } \\
\text { rhizome }\end{array}$ & Sore throats & General & Kayani et al. 2014 \\
\hline Persicaria chinensis (L.) H. Gross & Herb & $\begin{array}{l}\text { Root, } \\
\text { tubers, } \\
\text { leaves }\end{array}$ & Nerve damage; lymphnode inflammation; edible & General & Kanthasamy Kalaichelvi 2016 \\
\hline Persicaria glabra (Willd.) M. Gómez & Herb & Root, shoot & Piles; jaundice; constipation & General & Dhivya and Kalaichelvi 2015 \\
\hline Persicaria hydropiper (L.) Delarbre & Herb & Whole plant & Ringworm; fish poison; respiratory problems & General & $\begin{array}{l}\text { Jain 2004, Murty and Venkaiah } \\
2010\end{array}$ \\
\hline Persicaria punctata (Elliott) Small & Herb & Leaves & Swellings; head ache; body pain & General & Puravankara and Gopal 2012 \\
\hline Polygonum aviculare $\mathrm{L}$. & Herb & - & Anti-microbial & General & Zhang et al. 2013 \\
\hline Polygonum molle D. Don & Herb & Root & Tuberculosis & General & Dhanabal et al. 2015 \\
\hline Polygonum perfoliatum L. & Herb & Seed & - & General & Dey and De 2012 \\
\hline Polygonum plebeium $\mathrm{R} . \mathrm{Br}$. & Herb & Root & Inflammations & General & Shanmugam et al. 2012 \\
\hline Reynoutria japonica Houtt. & Herb & - & Anti-viral & General & Zhang et al. 2013 \\
\hline Rheum australe D. Don & Herb & $\begin{array}{l}\text { Stem, } \\
\text { rhizome, } \\
\text { root, leaves }\end{array}$ & Respiratory problems; asthma & General & Kayani et al. 2014 \\
\hline Rheum emodi Wall. & Herb & Root & Cuts and wounds & General & Kumar et al. 2007 \\
\hline Rheum officinale Baill. & Herb & - & Anti-tumour & General & Zhang et al. 2013 \\
\hline
\end{tabular}

Published 11 February 2019

http://dx.doi.org/10.32859/era.18.4.1-112 


\begin{tabular}{|c|c|c|c|c|c|}
\hline Rumex dentatus $\mathrm{L}$. & Herb & $\begin{array}{l}\text { Leaves, } \\
\text { root, stem }\end{array}$ & Cough; cold & General & Kayani et al. 2014 \\
\hline Rumex nepalensis Spreng. & Herb & Whole plant & Fracture; joint pain; edema; jaundice & General & Rajan et al. 1997 \\
\hline \multicolumn{6}{|l|}{ Polypodiaceae } \\
\hline Drynaria quercifolia (L.) J. Sm. & Fern & Whole plant & $\begin{array}{l}\text { Vomitng; arthralgia; migraine; rheumatism; typhoid; } \\
\text { anthelmintic; fever; dyspepsia; cough; typhoid; } \\
\text { abortifacient; fits; post partum problems; antibacterial }\end{array}$ & General & $\begin{array}{l}\text { Saravanan et al. 2013, Valsara } \\
\text { et al. } 1997\end{array}$ \\
\hline Microsorum punctatum (L.) Copel. & Fern & Leaves & Snakebite & General & Dey and De 2012 \\
\hline Pyrrosia heterophylla (L.) M.G. Price & $\begin{array}{l}\text { Epiphytes or } \\
\text { lithophytes } \\
\end{array}$ & Leaves, root & - & General & Xavier et al. 2014 \\
\hline Pyrrosia porosa (C. Presl) Hovenkamp & $\begin{array}{l}\text { Epiphytes or } \\
\text { lithophytes }\end{array}$ & $\begin{array}{l}\text { The scales } \\
\text { ground }\end{array}$ & Rheumatism & General & Rajendran et al. 2000 \\
\hline \multicolumn{6}{|l|}{ Polytrichaceae } \\
\hline Pogonatum macrophyllum Dozy and Molk. & Mosses & - & $\begin{array}{l}\text { Used to reduce inflammation and fever; also used as } \\
\text { detergent diuretic; laxative and hemostatic agent }\end{array}$ & $\begin{array}{l}\text { General; } \\
\text { women }\end{array}$ & Chandra et al. 2016 \\
\hline Polytrichum commune Hedw. & Mosses & - & $\begin{array}{l}\text { Used for hemostasis; wound healer; antipyretic; antidotal } \\
\text { activity; dissolve kidney and gall bladder stones; to speed } \\
\text { up labor process during child birth }\end{array}$ & $\begin{array}{l}\text { General; } \\
\text { pregnant } \\
\text { women }\end{array}$ & Chandra et al. 2016 \\
\hline Polytrichum juniperinum Hedw. & Mosses & - & $\begin{array}{l}\text { Used to treat prostate; uninary difficulties and skin } \\
\text { ailments }\end{array}$ & General & Chandra et al. 2016 \\
\hline \multicolumn{6}{|l|}{ Portulacaceae } \\
\hline Portulaca oleracea L. & Herb & Whole plant & $\begin{array}{l}\text { Mouth wound; vermifuge; liver and kidney stones; } \\
\text { cadiovascular diseases; earache; vegetable }\end{array}$ & General & $\begin{array}{l}\text { Dansi et al. 2008, Shanmugam } \\
\text { et al. } 2012\end{array}$ \\
\hline Portulaca pilosa L. & Herb & Whole plant & Diuretic & General & Jayanthi et al. 2012 \\
\hline Portulaca quadrifida L. & Herb & Whole plant & $\begin{array}{l}\text { Cold; flu; cough; cooling; diuretic; respiratory problems; } \\
\text { scorpion sting }\end{array}$ & General & Chopda and Mahajan 2009 \\
\hline $\begin{array}{l}\text { Portulaca wightiana Wall. ex Wight and } \\
\text { Arn. }\end{array}$ & Herb & Whole plant & Kidney problems; cardiovascular diseases & General & Kanthasamy Kalaichelvi 2016 \\
\hline \multicolumn{6}{|l|}{ Pottiaceae } \\
\hline Barbula convoluta Hedw. & Moss & Whole plant & - & General & Kennedy 2006 \\
\hline Hyophila involuta (Hook.) A. Jaeger & Moss & Leaves & Cold; cough and neck pain & General & Chandra et al. 2016 \\
\hline \multicolumn{6}{|l|}{ Primulaceae } \\
\hline Anagallis arvensis $\mathrm{L}$. & Herb & Whole plant & Skin disorder; fever; leprosy; cattle stomach & General & Wagh and Jain 2015 \\
\hline Ardisia solanacea (Poir.) Roxb. & Small tree & Root, bark & Cuts and wounds; Itching due to impure blood & General & Bhat et al. 2014 \\
\hline Embelia ribes Burm. $\mathrm{f}$. & Climber & Seed, fruit & Contraceptive; malaria & $\begin{array}{l}\text { General; } \\
\text { women }\end{array}$ & Chopda and Mahajan 2009 \\
\hline $\begin{array}{l}\text { Embelia tsjeriam-cottam (Roem. and } \\
\text { Schult.) A.DC. }\end{array}$ & Shrub & Leaves, root & Diarrhea; Mucolytic & General & Bhandary et al. 1995 \\
\hline Lysimachia christinae Hance & Herb & - & Anti-cancer & General & Zhang et al. 2013 \\
\hline Maesa indica (Roxb.) A.DC. & Shrub & $\begin{array}{l}\text { Leaves, } \\
\text { root, fruit }\end{array}$ & Disease resistance; hair growth & General & Bhagat et al. 2016 \\
\hline Primula macrophylla D. Don & Herb & $\begin{array}{l}\text { Flower, } \\
\text { leaves }\end{array}$ & Cough; asthma & General & Kayani et al. 2014 \\
\hline \multicolumn{6}{|l|}{ Proteaceae } \\
\hline Grevillea robusta A. Cunn. ex R. Br. & Tree & - & Agricultural tools & General & Prabakaran et al. 2013 \\
\hline
\end{tabular}

Published 11 February 2019

http://dx.doi.org/10.32859/era.18.4.1-112 


\begin{tabular}{|c|c|c|c|c|c|}
\hline \multicolumn{6}{|l|}{ Pteridaceae } \\
\hline Actiniopteris australis Link & Herb & Whole plant & Antibiotic; anti-fertility and antiseptic. & General & Jayanthi et al. 2012 \\
\hline Actiniopteris dichotoma Mett. & Fern & Whole plant & Typhoid & General & Porte 2014 \\
\hline Actiniopteris radiata (Sw.) Link & Fern & Whole plant & Bone fractures; rheumatism; asthma; psoriasis & General & $\begin{array}{l}\text { Jain et al. 2009, Rao et al. } \\
\text { 2006, Sharma et al. } 2013\end{array}$ \\
\hline Adiantum capillus-veneris $\mathrm{L}$. & Fern & Whole plant & Piles and jaundice; cold; Bronchial problems; cough & General & $\begin{array}{l}\text { Kayani et al. 2014, Rao et al. } \\
2006\end{array}$ \\
\hline Adiantum incisum Forssk. & Fern & Whole plant & Diabetes; migrane & General & Jayanthi et al. 2012 \\
\hline Adiantum lunulatum Burm. $\mathrm{f}$. & Fern & Whole plant & $\begin{array}{l}\text { Ovulation enhancement; abortifacient; Allergy; Cough; } \\
\text { Piles; Jaundice; epilepsy }\end{array}$ & General & $\begin{array}{l}\text { Bhogaonkar and Kadam 2006, } \\
\text { Das et al. 2014, Dey and De } \\
\text { 2012, Krishna et al. } 2011\end{array}$ \\
\hline Adiantum venustum D. Don & Fern & Whole plant & Lungs disorders & General & Kayani et al. 2014 \\
\hline Ceratopteris thalictroides (L.) Brongn. & Fern & Whole plant & Memory treatment; jaundice & General & $\begin{array}{l}\text { Asha and Pushpangadan 2002, } \\
\text { Sreeramulu et al. } 2013\end{array}$ \\
\hline Hemionitis arifolia (Burm. f.) T. Moore & Fern & Leaves & Skin disease; to cure rabies infection & General & Ayyanar and Ignacimuthu 2005 \\
\hline \multicolumn{6}{|l|}{ Putranjivaceae } \\
\hline $\begin{array}{l}\text { Drypetes assamica (Hook. f.) Pax and K. } \\
\text { Hoffm. }\end{array}$ & Tree & Leaves & - & - & Dey and De 2012 \\
\hline Putranjiva roxburghii Wall. & Tree & Bark & Cough & General & Sreeramulu et al. 2013 \\
\hline \multicolumn{6}{|l|}{ Ranunculaceae } \\
\hline Aconitum ferox Wall. ex Ser. & Herb & Tuber & Poison to kill rats and wild animals & General & Ruba and Mohan 2016 \\
\hline Aconitum heterophyllum Wall. ex Royle & Herb & Whole plant & Cold; cough; General & General & $\begin{array}{l}\text { Dey and De 2012, Kayani et al. } \\
2014\end{array}$ \\
\hline Aconitum violaceum Jacquem. ex Stapf & Herb & Root, flower & Cold; cough & General & Kayani et al. 2014 \\
\hline Aconitum ferox Wall. ex Ser. & Herb & Root & Malaria tratement & General & $\begin{array}{l}\text { Chenniappan and Kadarkarai } \\
2010\end{array}$ \\
\hline Actaea racemosa $\mathrm{L}$. & Herb & $\begin{array}{l}\text { Leaves, } \\
\text { root, fruit }\end{array}$ & $\begin{array}{l}\text { Bbronchitis; asthma and cough; sour throat; acidity; blood } \\
\text { purifier; pickled }\end{array}$ & General & Das and Swamy 2016 \\
\hline Actaea spicata L. & Herb & $\begin{array}{l}\text { Flower, root, } \\
\text { seed }\end{array}$ & 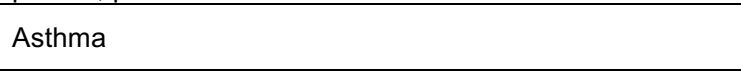 & General & Kayani et al. 2014 \\
\hline Clematis flammula L. & Climber & Leaves & To burn the warts & General & Parada et al. 2009 \\
\hline Clematis gouriana Roxb. ex DC. & Climber & Whole plant & $\begin{array}{l}\text { Skin diseases; wounds; lice killing; cuts; burns; worm } \\
\text { infection; veneral disease; skin burns; fever; musculo } \\
\text { skeletal disorder }\end{array}$ & General & Deepak and Gopal 2014 \\
\hline Clematis roylei Rehder & Climber & Leaves & Skin diseases like leucoderma & General & $\begin{array}{l}\text { Muralidhara Rao and Pullaiah } \\
2007\end{array}$ \\
\hline Clematis smilacifolia Wall. & Climber & $\begin{array}{l}\text { Rhizome, } \\
\text { leaves }\end{array}$ & $\begin{array}{l}\text { Rhizome paste mixed With breast milk; administered orally } \\
\text { to infants to cure stomach-ache; fever }\end{array}$ & Infants & $\begin{array}{l}\text { Muralidhara Rao and Pullaiah } \\
2007\end{array}$ \\
\hline Clematis wightiana Wall. & Climber & Leaves & Rheumatism & General & $\begin{array}{l}\text { Muralidhara Rao and Pullaiah } \\
2007\end{array}$ \\
\hline Naravelia zeylanica (L.) DC. & Climber & Whole plant & $\begin{array}{l}\text { Rheumatism; cold; headache; skin allergy; itch; skin } \\
\text { disease; chest pain; leprosy; buccal cavity sore; scabies; } \\
\text { eczema }\end{array}$ & General & Udayan et al. 2005 \\
\hline Nigella sativa $\mathrm{L}$. & Herb & Seed & To increase lactation & Women & Sayed et al. 2007 \\
\hline Ranunculus sceleratus L. & Herb & Whole plant & - & General & Chopda and Mahajan 2009 \\
\hline
\end{tabular}

Published 11 February 2019

http://dx.doi.org/10.32859/era.18.4.1-112 


\begin{tabular}{|c|c|c|c|c|c|}
\hline Thalictrum foliolosum DC. & Herb & Tuber & Hydrocele; Jaundice & General & Rao et al. 2011 \\
\hline \multicolumn{6}{|l|}{ Resedaceae } \\
\hline Ochradenus baccatus Delile & Shrub & Leaves & - & - & Chopda and Mahajan 2009 \\
\hline \multicolumn{6}{|l|}{ Rhamnaceae } \\
\hline Gouania leptostachya DC. & Climber & Root & Fever & General & Prasad et al. 2008 \\
\hline Gouania microcarpa DC. & Climber & Root & Body pain & General & Binu 2011 \\
\hline Paliurus spina-christi Mill. & Shrub & Fruit & To low cholesterol & General & Parada et al. 2009 \\
\hline Scutia myrtina (Burm.f.) Kurz & Climber & Leaves, fruit & Dysentery; edible & General & Kanthasamy Kalaichelvi 2016 \\
\hline Ventilago maderaspatana Gaertn. & Climber & Root, fruit & Stomach ache; fruit & General & Sreeramulu et al. 2013 \\
\hline Ziziphus abyssinica Hochst. ex A. Rich. & Shrub & Bark & Tuberculosis; anticancerous; antibiotic & General & Dhanabal et al. 2015 \\
\hline Ziziphus caracutta Buch. -Ham. ex Roxb. & $\begin{array}{l}\text { Erect shrub/ } \\
\text { small tree }\end{array}$ & Fruit & Edible & General & Bhagat et al. 2016 \\
\hline Ziziphus glabrata B. Heyne ex Roth & Tree & Fruit & Edible & General & Rekka and Kumar 2014 \\
\hline Ziziphus jujuba Mill. & Tree & Whole plant & $\begin{array}{l}\text { Cough; mouth cleanser; improves digestion; edible; } \\
\text { antidiarrhoeal; diarrhoea; dysentery; edible; wound; piles; } \\
\text { prolonged labour; dental caries; tuberculosis; ulcers; } \\
\text { asthma; constipation; diabetes }\end{array}$ & $\begin{array}{l}\text { General; } \\
\text { women }\end{array}$ & $\begin{array}{l}\text { Ayyanar and Ignacimuthu 2010, } \\
\text { Bhagat et al. 2016, Tetali et al. } \\
2009\end{array}$ \\
\hline $\begin{array}{l}\text { Ziziphus nummularia (Burm. f.) Wight and } \\
\text { Arn. }\end{array}$ & Climber & $\begin{array}{l}\text { Root, stem, } \\
\text { bark, leaves }\end{array}$ & $\begin{array}{l}\text { Rheumatism; fever; ulcer; inflammation; diarrhoea; } \\
\text { insecticidal; wormicidal; skin diseases }\end{array}$ & General & Bhardwaj et al. 2011 \\
\hline Ziziphus oenopolia (L.) Mill. & $\begin{array}{l}\text { Straggling or } \\
\text { climbing } \\
\text { shrub }\end{array}$ & $\begin{array}{l}\text { Fruit, bark, } \\
\text { root, leaves }\end{array}$ & $\begin{array}{l}\text { Amenorrhegia; dysentery; venereal diseases; diarrhoea; } \\
\text { wound; edible; gynaecological disorders }\end{array}$ & $\begin{array}{l}\text { General; } \\
\text { women }\end{array}$ & $\begin{array}{l}\text { Bhagat et al. 2016, Sreeramulu } \\
\text { et al. } 2013\end{array}$ \\
\hline Ziziphus rugosa Lam. & $\begin{array}{l}\text { Scandent } \\
\text { shrub }\end{array}$ & Whole plant & $\begin{array}{l}\text { Carbuncle; menorrhea; syphilis; ulcerated tongue; boils; } \\
\text { bone fracture; dysentery; edible; loose motions; treatment } \\
\text { of white discharge; diabetes }\end{array}$ & General & $\begin{array}{l}\text { Bhagat et al. 2016, Bhat et al. } \\
2014\end{array}$ \\
\hline Ziziphus xylopyrus (Retz.) Willd. & $\begin{array}{l}\text { Shrub to } \\
\text { small tree }\end{array}$ & $\begin{array}{l}\text { Leaves, } \\
\text { fruit, seed, } \\
\text { bark }\end{array}$ & $\begin{array}{l}\text { Antibacterial; antidiarrhoeal; to check oogenesis; } \\
\text { snakebite; fever; wound; boils; skin rashes }\end{array}$ & $\begin{array}{l}\text { General; } \\
\text { women }\end{array}$ & Prabakaran et al. 2013 \\
\hline \multicolumn{6}{|l|}{ Rhizophoraceae } \\
\hline Carallia brachiata (Lour.) Merr. & Tree & Bark & Menstrual problems & Women & Prasad et al. 2014 \\
\hline \multicolumn{6}{|l|}{ Rosaceae } \\
\hline Cydonia oblonga Mill. & Tree & Fruit & For bellyache & General & Parada et al. 2009 \\
\hline Duchesnea indica (Jacks.) Focke & Herb & - & Anti-cancer & General & Zhang et al. 2013 \\
\hline Fragaria indica Wall. & Runner & Whole plant & Sore throats; tuberculosis & General & Kayani et al. 2014 \\
\hline Potentilla nepalensis Hook. & Herb & Root & Burns & General & Kumar et al. 2007 \\
\hline Prunus armeniaca $\mathrm{L}$. & $\begin{array}{l}\text { Medium } \\
\text { sized tree }\end{array}$ & Fruit, seed & Vitamin deficiency & General & Acharya and Kaphle 2015 \\
\hline Prunus avium (L.) L. & Tree & Fruit & For bellyache & General & Parada et al. 2009 \\
\hline Prunus cerasoides Buch. -Ham. ex D. Don & Tree & Bark & Body swellings & General & Kumar et al. 2011 \\
\hline Prunus cerasus L. & Tree & Seed & - & General & Chopda and Mahajan 2009 \\
\hline Prunus dulcis (Mill.) D.A. Webb & Tree & Whole plant & Impotency & General & Bhogaonkar and Kadam 2006 \\
\hline Prunus mahaleb L. & Tree & Root & - & General & Chopda and Mahajan 2009 \\
\hline Prunus persica (L.) Batsch & Tree & Leaves & Dysentry; wound & General & Gairola et al. 2013 \\
\hline Pyrus communis $\mathrm{L}$. & Tree & Fruit & Edible & General & Rekka and Kumar 2014 \\
\hline
\end{tabular}

Published 11 February 2019

http://dx.doi.org/10.32859/era.18.4.1-112 


\begin{tabular}{|c|c|c|c|c|c|}
\hline Rosa $\times$ damascena Herrm. & Shrub & Flower & Pimples & General & Jenisha and Jeeva 2014 \\
\hline Rosa chinensis Jacq. & Shrub & $\begin{array}{l}\text { Fruit, } \\
\text { leaves, } \\
\text { flower }\end{array}$ & Epistaxis & General & Chopda and Mahajan 2009 \\
\hline Rosa indica L. & Shrub & Leaves & - & General & Patel 2014 \\
\hline Rosa multiflora Thunb. & Shrub & Fruit & - & General & Chopda and Mahajan 2009 \\
\hline Rubus ellipticus Sm. & Shrub & $\begin{array}{l}\text { Aerial parts, } \\
\text { root }\end{array}$ & $\begin{array}{l}\text { Fits; leucorrhoea; menorrhagia; stomach pain; urinary tract } \\
\text { infection; edible; peptic ulcers; diabetes; inflammations; } \\
\text { sores; ulcers; antibacterial; edible }\end{array}$ & $\begin{array}{l}\text { General; } \\
\text { women }\end{array}$ & $\begin{array}{l}\text { Ajesh et al. 2012, Rao et al. } \\
2011\end{array}$ \\
\hline Sanguisorba officinalis L. & Herb & - & Antimicrobial & General & Zhang et al. 2013 \\
\hline \multicolumn{6}{|l|}{ Rubiaceae } \\
\hline Benkara malabarica (Lam.) Tirveng. & Small tree & $\begin{array}{l}\text { Leaves, } \\
\text { root, bark, } \\
\text { fruit }\end{array}$ & $\begin{array}{l}\text { Abdominal pain and throat infection; wound healing; } \\
\text { asthma; rheumatic pains }\end{array}$ & General & Kanthasamy Kalaichelvi 2016 \\
\hline $\begin{array}{l}\text { Canthium coromandelicum (Burm. f.) } \\
\text { Alston }\end{array}$ & $\begin{array}{l}\text { Medium } \\
\text { sized shrub }\end{array}$ & $\begin{array}{l}\text { Root, } \\
\text { leaves, bark, } \\
\text { fruit }\end{array}$ & $\begin{array}{l}\text { Fever; Diarrhoea; Insect bite; snakebite; Astringent; } \\
\text { diuretic; febrifuge; anthelmintic; constipation }\end{array}$ & General & $\begin{array}{l}\text { Mahishi et al. 2005, Padal et al. } \\
\text { 2010, Pradheeps and Poyyamoli } \\
2013\end{array}$ \\
\hline Catunaregam nutans (Roxb.) Tirveng. & $\begin{array}{l}\text { Shrub to } \\
\text { small tree }\end{array}$ & Root & Malarial treatment & General & Jain 2004 \\
\hline Catunaregam spinosa (Thunb.) Tirveng. & $\begin{array}{l}\text { Shrub to } \\
\text { small tree }\end{array}$ & $\begin{array}{l}\text { Fruit, } \\
\text { leaves, root, } \\
\text { bark, seed }\end{array}$ & Skin diseases; skin boils; sprains; Antispasmobic; asthma & General & $\begin{array}{l}\text { Kanthasamy Kalaichelvi 2016, } \\
\text { Kshirsagar and Singh 2001, } \\
\text { Ragupathy and Newmaster } \\
2009\end{array}$ \\
\hline Ceriscoides turgida (Roxb.) Tirveng. & Tree & Bark, seed & Piles; tympany; diarrhoea; leucorrhoea; food poisoning & General & Sreeramulu et al. 2013 \\
\hline Chassalia curviflora (Wall.) Thwaites & Shrub & Leaves & Wounds caused by snakebites & General & Dey and De 2012 \\
\hline Coelospermum decipiens Baill. & $\begin{array}{l}\text { Climbing } \\
\text { shrub }\end{array}$ & Whole plant & - & - & Xavier et al. 2014 \\
\hline Fleroya stipulosa (DC.) Y.F. Deng & Tree & Leaves & Vegetable & General & Dansi et al. 2008 \\
\hline Galium aparine L. & Herb & Whole plant & Cuts & General & Kumar et al. 2007 \\
\hline Galium asperuloides Edgew. & Herb & Whole plant & Epilepsy & General & Sharma et al. 2013 \\
\hline Galium rotundifolium L. & Herb & Whole plant & - & General & Sharma et al. 2013 \\
\hline Gardenia gummifera L.f. & Tree & Gum, bark & $\begin{array}{l}\text { Antiasthmatic; stomachache; skin diseases; stomach } \\
\text { ulcers }\end{array}$ & General & $\begin{array}{l}\text { Kumar et al. 2016, Rao et al. } \\
2006\end{array}$ \\
\hline Gardenia jasminoides J. Ellis & Shrub & - & Anaemia & General & Chithra et al. 2016 \\
\hline Gardenia latifolia Aiton & Tree & $\begin{array}{l}\text { Bark, } \\
\text { leaves, fruit }\end{array}$ & Piles; Boils; Blisters; Wounds; fever & General & Sreeramulu et al. 2013 \\
\hline Gardenia ternifolia Schumach. and Thonn. & Shrub & Leaves & Vegetable & General & Dansi et al. 2008 \\
\hline Haldina cordifolia (Roxb.) Ridsdale & Tree & Bark & $\begin{array}{l}\text { Stops over periods in women; contraceptive; stomach } \\
\text { ache }\end{array}$ & $\begin{array}{l}\text { General; } \\
\text { women }\end{array}$ & Ayyanar and Ignacimuthu 2010 \\
\hline Hedyotis scandens Roxb. & Climber & $\begin{array}{l}\text { Leaves, } \\
\text { twigs }\end{array}$ & Gastritis; Beverages fermentation & General & Ruba and Mohan 2016 \\
\hline Hymenodictyon obovatum Wall. & Tree & Bark & Amenorrhegia & Women & Bhogaonkar and Kadam 2006 \\
\hline Hymenodictyon orixense (Roxb.) Mabb. & Tree & Bark & Bone fracture; menstrual pain & $\begin{array}{l}\text { General; } \\
\text { women }\end{array}$ & Sreeramulu et al. 2013 \\
\hline
\end{tabular}

Published 11 February 2019

http://dx.doi.org/10.32859/era.18.4.1-112 


\begin{tabular}{|c|c|c|c|c|c|}
\hline Ixora brachiata Roxb. & Tree & Bark & inflammation & General & $\begin{array}{l}\text { Manikandan and Lakshmanan } \\
2014\end{array}$ \\
\hline Ixora coccinea L. & Small shrub & $\begin{array}{l}\text { Leaves, } \\
\text { flower, fruit, } \\
\text { root }\end{array}$ & $\begin{array}{l}\text { Wounds; skin ulcers; sore throut; Bronchitis cough; } \\
\text { Asthma; Tumor; jaundice; skin diseases; hepatoprotective; } \\
\text { cholera; conception; leucorrhea; scabies; antimicrobial; } \\
\text { antitumor; oral cancer }\end{array}$ & Children & $\begin{array}{l}\text { Asha and Pushpangadan 2002, } \\
\text { Kshirsagar and Singh 2001, } \\
\text { Nizar et al. } 2015\end{array}$ \\
\hline Ixora pavetta Andr. & Tree & $\begin{array}{l}\text { Root, bark, } \\
\text { wood, } \\
\text { leaves }\end{array}$ & $\begin{array}{l}\text { Hair tonic; headache; firewood; chest pain; leucoderma; } \\
\text { wound }\end{array}$ & General & $\begin{array}{l}\text { Bhat et al. 2014, Sreeramulu et } \\
\text { al. } 2013\end{array}$ \\
\hline Macrosphyra longistyla (DC.) Hiern & Shrub & Leaves & Vegetable & General & Dansi et al. 2008 \\
\hline Meyna laxiflora Robyns & $\begin{array}{l}\text { Shrub to } \\
\text { small tree }\end{array}$ & Fruit & Edible & General & Bhagat et al. 2016 \\
\hline Meyna spinosa Roxb. ex Link & $\begin{array}{l}\text { Straggling } \\
\text { shrub }\end{array}$ & Fruit & Abortion & General & Murty and Venkaiah 2010 \\
\hline Mitracarpus hirtus (L.) DC. & Herb & Fruit, leaves & Skin diseases; wound healing; diarrhoea & General & Kanthasamy Kalaichelvi 2016 \\
\hline Mitragyna parvifolia (Roxb.) Korth. & Tree & $\begin{array}{l}\text { Leaves, } \\
\text { fruit, bark, } \\
\text { root, stem } \\
\text { bark }\end{array}$ & Snake bite; insecticidal; wormicidal; rheumatism & General & $\begin{array}{l}\text { Padma et al. 2016, Rao et al. } \\
2006\end{array}$ \\
\hline Morinda angustifolia Roxb. & $\begin{array}{l}\text { Medium } \\
\text { sized tree }\end{array}$ & Root, stem & Treating gastric ulcer; obstetric diseases & General & Srithi et al. 2009 \\
\hline Morinda citrifolia L. & Tree & Leaves & - & General & Chopda and Mahajan 2009 \\
\hline Morinda coreia Buch. -Ham. & Small tree & $\begin{array}{l}\text { Bark, fruit, } \\
\text { leaves }\end{array}$ & $\begin{array}{l}\text { Diabetes; cardiovascular disease; cuts; wound; diarrhoea; } \\
\text { ulcer }\end{array}$ & General & Bosco and Arumugam 2012 \\
\hline Morinda pubescens Sm. & Tree & Bark, leaves & Typhoid; earache; wound & General & Ignacimuthu et al. 2008 \\
\hline Mussaenda frondosa $\mathrm{L}$. & Shrub & Leaves & To induce sound sleep & General & Pushpangadan and Atal 1984 \\
\hline $\begin{array}{l}\text { Mussaenda hirsutissima (Hook. f.) Hutch. } \\
\text { ex Gamble }\end{array}$ & Shrub & $\begin{array}{l}\text { Leaves, } \\
\text { stem }\end{array}$ & $\begin{array}{l}\text { To cure heel cracks; to cure sterility in both men and } \\
\text { women }\end{array}$ & $\begin{array}{l}\text { General; } \\
\text { women; } \\
\text { men }\end{array}$ & Ayyanar and Ignacimuthu 2005 \\
\hline Neanotis indica (DC.) W.H. Lewis & Herb & Leaves & Scorpion and insect bites & General & Manikandan 2008 \\
\hline Neolamarckia cadamba (Roxb.) Bosser & Tree & $\begin{array}{l}\text { Stem, fruit, } \\
\text { bark, leaves }\end{array}$ & Snakebite; urinary tract infections; measles & General & Dubey et al. 2012 \\
\hline Neonauclea purpurea (Roxb.) Merr. & Tree & $\begin{array}{l}\text { Leaves, } \\
\text { bark }\end{array}$ & For sleeping & General & Dey and De 2012 \\
\hline $\begin{array}{l}\text { Oldenlandia affinis (Roem. and Schult.) } \\
\text { DC. }\end{array}$ & Herb & Whole plant & Antiageing; menstrual problems & $\begin{array}{l}\text { General; } \\
\text { women }\end{array}$ & Rajendran et al. 2002 \\
\hline Oldenlandia auricularia (L.) K. Schum. & Trailing herb & Whole plant & Cholera & General & Mahishi et al. 2005 \\
\hline Oldenlandia biflora L. & Herb & Whole plant & - & - & Chopda and Mahajan 2009 \\
\hline Oldenlandia corymbosa L. & Herb & Whole plant & Antibacterial & General & Valsaraj et al. 1997 \\
\hline Oldenlandia diffusa (Willd.) Roxb. & Herb & Whole plant & Antimicrobial; snakebite & General & Samy et al. 2008 \\
\hline Oldenlandia umbellata L. & Herb & Leaves, root & Snakebite; asthma; bronchitis; worms & General & $\begin{array}{l}\text { Kumar et al. 2007, Shanmugam } \\
\text { et al. } 2012\end{array}$ \\
\hline Ophiorrhiza mungos L. & Herb & Root & Snake bite & General & Samy et al. 2008 \\
\hline Paederia foetida L. & Shrub & Whole plant & Rheumatism; dysentery; stomachache & General & Sreeramulu et al. 2013 \\
\hline
\end{tabular}

Published 11 February 2019

http://dx.doi.org/10.32859/era.18.4.1-112 
Ethnobotany Research and Applications

\begin{tabular}{|c|c|c|c|c|c|}
\hline Pavetta breviflora DC. & $\begin{array}{l}\text { Shrub to } \\
\text { small tree }\end{array}$ & Bark & Snakebite & General & Raju 2008 \\
\hline Pavetta indica L. & $\begin{array}{l}\text { Shrub to } \\
\text { small tree }\end{array}$ & $\begin{array}{l}\text { Root, } \\
\text { leaves, bark, } \\
\text { fruit }\end{array}$ & $\begin{array}{l}\text { Ophthalmic diseases; epilepsy; jaundice; indigestion; } \\
\text { eczema; edible }\end{array}$ & General & $\begin{array}{l}\text { Padal et al. 2010, Ratna Manjula } \\
\text { et al. } 2011\end{array}$ \\
\hline $\begin{array}{l}\text { Pentodon pentandrus (Schumach. and } \\
\text { Thonn.) Vatke }\end{array}$ & $\begin{array}{l}\text { Straggling } \\
\text { herb }\end{array}$ & Leaves & Vegetable & General & Dansi et al. 2008 \\
\hline Psychotria dalzellii Hook. f. & $\begin{array}{l}\text { Shrub to } \\
\text { small tree }\end{array}$ & Stem & Pruritus & General & Bhat et al. 2014 \\
\hline Psychotria flavida Talbot & Shrub & Root & Snake-bite & General & Kshirsagar and Singh 2001 \\
\hline Psychotria platyneura Kurz & Shrub & Bark & Skin diseases & General & Prasad et al. 2008 \\
\hline Psydrax dicoccos Gaertn. & Tree & $\begin{array}{l}\text { Wood, } \\
\text { leaves, fruit, } \\
\text { bark }\end{array}$ & $\begin{array}{l}\text { Bone fractures; dandruff; burns; edible; fever; firewood; } \\
\text { diarrhoea }\end{array}$ & General & $\begin{array}{l}\text { Ram et al. 2004, Ratnam and } \\
\text { Raju } 2008\end{array}$ \\
\hline Psydrax umbellata (Wight) Bridson & Tree & Leaves & Kidney and bladder ailments & General & Kanthasamy Kalaichelvi 2016 \\
\hline Richardia scabra L. & Herb & Leaves & Skin diseases & General & Ayyanar and Ignacimuthu 2005 \\
\hline Rubia cordifolia L. & Climber & Whole plant & $\begin{array}{l}\text { Abortifacient; epilepsy; gynaecological disorders; jaundice; } \\
\text { leucorrhea; restorative tonic; skin diseases; liver diseases }\end{array}$ & $\begin{array}{l}\text { General; } \\
\text { pregnant } \\
\text { women; } \\
\text { women }\end{array}$ & $\begin{array}{l}\text { Chopda and Mahajan 2009, Jain } \\
2004\end{array}$ \\
\hline Spermacoce articularis L.f. & Herb & Leaves, fruit & Wound; ear-ache; sore legs & General & $\begin{array}{l}\text { Latheef et al. 2014, Rao et al. } \\
2006\end{array}$ \\
\hline Spermacoce hispida L. & Herb & Whole plant & $\begin{array}{l}\text { Malaria; kidney stones; nervous disorders; skin bulging; } \\
\text { reduces body weight; stomach problems; improves vitality; } \\
\text { purifies blood }\end{array}$ & General & $\begin{array}{l}\text { Pushpangadan and Atal } 1984, \\
\text { Wagh and Jain } 2015\end{array}$ \\
\hline Spermacoce neohispida Govaerts & $\begin{array}{l}\text { Herb to } \\
\text { small shrub }\end{array}$ & Leaves & Wound healing & General & Dey et al. 2012 \\
\hline Spermacoce ocymoides Burm. f. & Herb & Leaves & Wound healing; insect sting & General & $\begin{array}{l}\text { Ayyanar and Ignacimuthu 2005, } \\
\text { Rajendran et al. } 2000\end{array}$ \\
\hline Spermacoce verticillata $\mathrm{L}$. & Shrub & Root & Leucorrhoea & General & $\begin{array}{l}\text { Manikandan and Lakshmanan } \\
2014\end{array}$ \\
\hline $\begin{array}{l}\text { Tamilnadia uliginosa (Retz.) Tirveng. and } \\
\text { Sastre }\end{array}$ & Tree & Bark, fruit & Bone fractures; diarrhoea; dysentery; boils; edible & General & $\begin{array}{l}\text { Bhagat et al. 2016, Ratnam and } \\
\text { Raju } 2008\end{array}$ \\
\hline Tarenna asiatica (L.) Kuntze ex K. Schum. & $\begin{array}{l}\text { Shrub to } \\
\text { small tree }\end{array}$ & Root, leaves & Cuts and boils; fits & General & Sreeramulu et al. 2013 \\
\hline Tarenna odorata (Roxb.) B.L. Rob. & $\begin{array}{l}\text { Shrub to } \\
\text { small tree }\end{array}$ & Root & Snakebite & General & Dey and De 2012 \\
\hline $\begin{array}{l}\text { Uncaria rhynchophylla (Miq.) Miq. ex } \\
\text { Havil. }\end{array}$ & Climber & - & Antipyretic; anti-hypertensive; anticonvulsant & General & Zhang et al. 2013 \\
\hline Wendlandia thyrsoidea (Roth) Steud. & $\begin{array}{l}\text { Shrub to } \\
\text { small tree }\end{array}$ & Root & Skin disease & General & Kshirsagar and Singh 2001 \\
\hline \multicolumn{6}{|l|}{ Rutaceae } \\
\hline Aegle marmelos (L.) Corrêa & Tree & Whole plant & $\begin{array}{l}\text { For cooling of body; blood sugar reduction; gastric } \\
\text { problems; eye problems; fever; influenza; sexual debility; } \\
\text { skin boils; diabetes; Gastricproblems; Respiratory }\end{array}$ & General & $\begin{array}{l}\text { Bhardwaj et al. 2011, Harsha et } \\
\text { al. 2003, Jain et al. 2004, } \\
\text { Kosalge and Fursule 2009, }\end{array}$ \\
\hline
\end{tabular}

Published 11 February 2019

http://dx.doi.org/10.32859/era.18.4.1-112 


\begin{tabular}{|c|c|c|c|c|c|}
\hline & & & $\begin{array}{l}\text { disorder; dyspepsia; anti inflammatory; loose motions; } \\
\text { snake bite; liver disease; diarrhoea; laxative; eye disease; } \\
\text { to treat malaria and malaria-like symptoms }\end{array}$ & & $\begin{array}{l}\text { Mahishi et al. 2005, Reddy et al. } \\
2008 \text {, Samy et al. } 2008\end{array}$ \\
\hline Atalantia monophylla DC. & Tree & $\begin{array}{l}\text { Leaves, } \\
\text { root, fruit }\end{array}$ & $\begin{array}{l}\text { rheumatoid pain and glandular swelling; itch; skin } \\
\text { complaints; dysentery; blood purifier; paralytic limb; } \\
\text { stomach disorder; antiseptic; snake bite; cough; } \\
\text { rheumatism pain }\end{array}$ & General & $\begin{array}{l}\text { Arjunan et al. 2016, Kanthasamy } \\
\text { Kalaichelvi } 2016\end{array}$ \\
\hline Atalantia racemosa Wight ex Hook. & Tree & $\begin{array}{l}\text { Leaves, } \\
\text { root, fruit }\end{array}$ & Fever; allergy & General & Harsha et al. 2002 \\
\hline Chloroxylon swietenia DC. & Tree & Whole plant & $\begin{array}{l}\text { Scorpion sting; shivering; neck pain; itchy skin; firewood; } \\
\text { cuts; cattle wounds; chest pain; stomach pain; coagulation } \\
\text { plant; epilepsy; fever; tooth problems; arthritis }\end{array}$ & $\begin{array}{l}\text { General; } \\
\text { cattle }\end{array}$ & Raju 2008 \\
\hline Citrus $\times$ aurantium $\mathrm{L}$. & Small tree & Fruit & Immunity; vit C; diabetes & General & Lingaraju et al. 2013 \\
\hline Citrus aurantiifolia (Christm.) Swingle & $\begin{array}{l}\text { Shrub to } \\
\text { small tree }\end{array}$ & Fruit, leaves & $\begin{array}{l}\text { Skin irritation; nausea; scurvy; decreased appetite; } \\
\text { stomach diseases; biliousness; sore throat and eye } \\
\text { disorder; catarrhal fever; headache; edible; boils; } \\
\text { diarrhoea; low BP }\end{array}$ & General & $\begin{array}{l}\text { Bhat et al. 2014, Ignacimuthu et } \\
\text { al. } 2008\end{array}$ \\
\hline Citrus indica Yu. Tanaka & Tree & Fruit, seed & Face pimples removal & General & Ruba and Mohan 2016 \\
\hline Citrus limon (L.) Osbeck & Tree & Fruit & $\begin{array}{l}\text { Dandruff; skin rashes; to reduce obesity; bone fracture; } \\
\text { snake bite; vit C }\end{array}$ & General & $\begin{array}{l}\text { Samy et al. 2008, Upadhya et al. } \\
2012\end{array}$ \\
\hline Citrus maxima (Burm.) Merr. & Tree & $\begin{array}{l}\text { Bark, fruit, } \\
\text { oil }\end{array}$ & Vitamin C source; edible; cough & General & Kayani et al. 2014 \\
\hline Citrus medica L. & Tree & $\begin{array}{l}\text { Leaves, } \\
\text { fruit, stem } \\
\text { bark }\end{array}$ & $\begin{array}{l}\text { Wound healing; head ache; tooth ache; Vitamin C source; } \\
\text { joint pain; indigestion; headache; bone fracture; nail } \\
\text { infection; refreshner }\end{array}$ & General & $\begin{array}{l}\text { Kshirsagar and Singh 2001, } \\
\text { Muralidhara Rao and Pullaiah } \\
\text { 2007, Sharma et al. } 2013\end{array}$ \\
\hline Citrus reticulata Blanco & Tree & Leaves & Flu & General & Kayani et al. 2014 \\
\hline Citrus sinensis (L.) Osbeck & Tree & Seed & To treat malaria and malaria-like symptoms & General & $\begin{array}{l}\text { Chenniappan and Kadarkarai } \\
2010\end{array}$ \\
\hline $\begin{array}{l}\text { Clausena anisata (Willd.) Hook. f. ex } \\
\text { Benth. }\end{array}$ & Shrub & Leaves, fruit & Mosquitoe repellent; eczema; wound healing & General & $\begin{array}{l}\text { Muralidhara Rao and Pullaiah } \\
2007 \text {, Ramya } 2008\end{array}$ \\
\hline $\begin{array}{l}\text { Clausena austroindica B.C. Stone and } \\
\text { K.K.N. Nair }\end{array}$ & Small tree & Root & Ear infection & General & $\begin{array}{l}\text { Muralidhara Rao and Pullaiah } \\
2007\end{array}$ \\
\hline Glycosmis pentaphylla (Retz.) DC. & Shrub & Whole plant & $\begin{array}{l}\text { Stomachache; abdominal discomfort; antisnake venom; } \\
\text { leucorrhea; piles; cough; rheumatism; anaemia; jaundice; } \\
\text { dandruff; malaria; fits(pregnant women); convulsions }\end{array}$ & $\begin{array}{l}\text { General; } \\
\text { women; } \\
\text { pregnant } \\
\text { women }\end{array}$ & $\begin{array}{l}\text { Bhagat et al. 2016, } \\
\text { Pushpangadan and Atal 1984, } \\
\text { Savithramma et al. } 2014\end{array}$ \\
\hline Limonia acidissima Groff & Tree & $\begin{array}{l}\text { Leaves, } \\
\text { bark, fruit, } \\
\text { rhizome }\end{array}$ & $\begin{array}{l}\text { Insect bites; diarrhoea; dysentery; snake bite; diabetes; } \\
\text { rheumatoid arthritis; breathing trouble; cuts }\end{array}$ & General & $\begin{array}{l}\text { Arjunan et al. 2016, Rao et al. } \\
2006\end{array}$ \\
\hline $\begin{array}{l}\text { Luvunga scandens (Roxb.) Buch. -Ham. } \\
\text { ex Wight and Arn. }\end{array}$ & Shrub & Root & - & - & Chopda and Mahajan 2009 \\
\hline $\begin{array}{l}\text { Melicope lunu-ankenda (Gaertn.) T.G. } \\
\text { Hartley }\end{array}$ & Tree & Root & Asthma and bronchitis & General & $\begin{array}{l}\text { Muralidhara Rao and Pullaiah } \\
2007\end{array}$ \\
\hline Murraya koenigii (L.) Spreng. & $\begin{array}{l}\text { Medium } \\
\text { sized tree }\end{array}$ & Whole plant & $\begin{array}{l}\text { Dysentery; vomiting; febrifuge; tonic; skin diseases; } \\
\text { antidiabetic; inflammations; jaundice; stomach pain; }\end{array}$ & $\begin{array}{l}\text { General; } \\
\text { women }\end{array}$ & $\begin{array}{l}\text { Ayyanar 2013, Kadhirvel et al. } \\
\text { 2010, Ratna Manjula et al. } 2011\end{array}$ \\
\hline
\end{tabular}

Published 11 February 2019

http://dx.doi.org/10.32859/era.18.4.1-112 


\begin{tabular}{|c|c|c|c|c|c|}
\hline & & & $\begin{array}{l}\text { indigestion; hair loss; dogbite; cough; cold; eye problems; } \\
\text { rheumatism; menstural problems; conceptions; diarrhoea }\end{array}$ & & \\
\hline Murraya paniculata (L.) Jack & Shrub & $\begin{array}{l}\text { Leaves, } \\
\text { bark }\end{array}$ & $\begin{array}{l}\text { Dropsy; body ache; fever; inflammation; bone fracture; } \\
\text { paralysis; mouth ulcers; diabetes; antifertility; analgesic; } \\
\text { antibacterial }\end{array}$ & General & $\begin{array}{l}\text { Chakraborty et al. } 2012, \\
\text { Lingaraju et al. } 2013\end{array}$ \\
\hline Naringi crenulata (Roxb.) Nicolson & Small tree & $\begin{array}{l}\text { Root, stem, } \\
\text { bark, fruit, } \\
\text { leaves, seed }\end{array}$ & $\begin{array}{l}\text { Piles; cobrabite; bodypain; colic; vomiting and dysentery; } \\
\text { acne and anti aging; fever; pitta; sprain; mental disorders; } \\
\text { dysentry; digestive disorders; epilepsy; intestinal worms; } \\
\text { cnthelmintic; antidote for insect poison; cooling agent; leg } \\
\text { pains }\end{array}$ & General & Kumar et al. 2007 \\
\hline Paramignya monophylla Wight & Tree & Root & Boils & General & Bhat et al. 2014 \\
\hline $\begin{array}{l}\text { Pleiospermium alatum (Wight and Arn.) } \\
\text { Swingle }\end{array}$ & Small tree & Whole plant & Anti-inflammatory; anti-septic; chest pain & General & Pradheeps and Poyyamoli 2013 \\
\hline Ruta chalepensis $\mathrm{L}$. & Herb & Leaves & Abortion; protects from convulsions; cough; constipation & $\begin{array}{l}\text { General; } \\
\text { children; } \\
\text { pregnant } \\
\text { women }\end{array}$ & Parada et al. 2009 \\
\hline Ruta graveolens $\mathrm{L}$. & Herb & Leaves & Typhoid; antibacterial; rheumatic pain; fever; skin diseases & General & $\begin{array}{l}\text { Dey and De 2012, Sharma et al. } \\
2013\end{array}$ \\
\hline Toddalia asiatica (L.) Lam. & Climber & Whole plant & $\begin{array}{l}\text { Boils; blisters; cuts; diarrhoea; fever; headache; } \\
\text { indigestion; cough; influenza; lung diseases; rheumatism; } \\
\text { malaria; cholera; skin diseases; snakebite; stomachache; } \\
\text { toothache; antiinflammatory; asthma; cough }\end{array}$ & $\begin{array}{l}\text { General; } \\
\text { Cow; goat }\end{array}$ & $\begin{array}{l}\text { Duraipandiyan et al. 2006, } \\
\text { Kshirsagar and Singh } 2001\end{array}$ \\
\hline Zanthoxylum ovalifolium Tutcher & Tree & Root, seed & Constipation; swolen joints & General & $\begin{array}{l}\text { Muralidhara Rao and Pullaiah } \\
2007\end{array}$ \\
\hline Zanthoxylum rhetsa DC. & Tree & $\begin{array}{l}\text { Seed, bark, } \\
\text { fruit }\end{array}$ & Typhoid weakness; bone fracture; edible & General & Nanjunda 2010 \\
\hline \multicolumn{6}{|l|}{ Salicaceae } \\
\hline Casearia elliptica Tul. & Tree & $\begin{array}{l}\text { Root, bark, } \\
\text { leaves, fruit }\end{array}$ & $\begin{array}{l}\text { Corneal opacity; Ephemeral fever; Aphrodisiac; Muscular } \\
\text { pain; Anti helminthic }\end{array}$ & General & $\begin{array}{l}\text { Rao et al. 2011, Sreeramulu et } \\
\text { al. } 2013\end{array}$ \\
\hline Casearia tomentosa Roxb. & Tree & Leaves, fruit & Bone fracture; edible & General & Upadhya et al. 2012 \\
\hline Flacourtia indica (Burm. f.) Merr. & $\begin{array}{l}\text { Shrub to } \\
\text { small tree }\end{array}$ & $\begin{array}{l}\text { Stem, root, } \\
\text { leaves, fruit }\end{array}$ & $\begin{array}{l}\text { Boils; blisters; cuts; epilepsy; snakebite; liver diseases; } \\
\text { digestive problems }\end{array}$ & General & Savithramma et al. 2014 \\
\hline Flacourtia montana J. Graham & Tree & Fruit & Edible & General & Bhagat et al. 2016 \\
\hline Homalium nepalense Benth. & Tree & Bark & Puerperal fever & General & Rao et al. 2011 \\
\hline Salix tetrasperma Roxb. & Tree & Stem, flower & - & General & Chopda and Mahajan 2009 \\
\hline \multicolumn{6}{|l|}{ Salvadoraceae } \\
\hline Azima tetracantha Lam. & $\begin{array}{l}\text { Rambling } \\
\text { shrub }\end{array}$ & Bark, leaves & $\begin{array}{l}\text { Infant diseases; Rheumatism; fever; cold cough; wound; } \\
\text { oral health care }\end{array}$ & General & $\begin{array}{l}\text { Hebbar et al. 2004, Raghupathy } \\
\text { and Newmaster } 2009\end{array}$ \\
\hline Salvadora aculeate L. & Tree & Aerial parts & Astringent; antiseptic; anti-fungal & General & Kanthasamy Kalaichelvi 2016 \\
\hline Salvadora persica L. & $\begin{array}{l}\text { Shrub to } \\
\text { small tree }\end{array}$ & $\begin{array}{l}\text { Fruit, root, } \\
\text { bark, leaves, } \\
\text { flower }\end{array}$ & $\begin{array}{l}\text { Snakebite; rheumatism; scurvy; fever; skin irritation; } \\
\text { purgative; antitumor; cold; cough; body pain }\end{array}$ & General & $\begin{array}{l}\text { Kosalge and Fursule 2009, } \\
\text { Padma et al. 2016, Ragupathy } \\
\text { and Newmaster } 2009\end{array}$ \\
\hline \multicolumn{6}{|l|}{ Santalaceae } \\
\hline Osyris lanceolata Hochst. and Steud. & Shrub & Leaves & Wounds & General & Puravankara and Gopal 2012 \\
\hline
\end{tabular}

Published 11 February 2019

http://dx.doi.org/10.32859/era.18.4.1-112 


\begin{tabular}{|c|c|c|c|c|c|}
\hline Santalum album L. & Tree & $\begin{array}{l}\text { Bark, root, } \\
\text { wood, seed } \\
\text { oil, fruit }\end{array}$ & $\begin{array}{l}\text { Body cooling; diarrhoea; dysentery; pruritus; fever; } \\
\text { indigestion; giddiness; cough; gonorrhea; headache; joint } \\
\text { pains; herpes; pimples; urinary infections; skin diseases; } \\
\text { throat ulcers; blood purifier }\end{array}$ & General & $\begin{array}{l}\text { Ignacimuthu et al. 2008, Mahish } \\
\text { et al. } 2005, \text { Pushpangadan and } \\
\text { Atal } 1984\end{array}$ \\
\hline Scleropyrum pentandrum (Dennst.) Mabb. & Tree & $\begin{array}{l}\text { Stem, } \\
\text { leaves }\end{array}$ & Skin diseases & General & Ayyanar and Ignacimuthu 2005 \\
\hline Viscum album L. & Shrub & Whole plant & - & General & Chopda and Mahajan 2009 \\
\hline Viscum angulatum B. Heyne ex DC. & Shrub & Whole plant & Skin cuts & General & Kshirsagar and Singh 2001 \\
\hline Viscum articulatum Burm. f. & Herb & Whole plant & Abortion; snakebite & General & Murty and Venkaiah 2010 \\
\hline Viscum coloratum (Kom.) Nakai & Tree & - & Anti-tumour & General & Zhang et al. 2013 \\
\hline Viscum monoicum Roxb. ex DC. & Herb & - & Typhoid & General & Porte 2014 \\
\hline Viscum trilobatum Talbot & Herb & Leaves & Cough and cold & General & Dhivya and Kalaichelvi 2015 \\
\hline \multicolumn{6}{|l|}{ Sapindaceae } \\
\hline Aesculus assamica Griff. & Tree & Bark & Fish poison & General & Ruba and Mohan 2016 \\
\hline Cardiospermum corindum $\mathrm{L}$. & Herb & Whole plant & Joint pain; rheumatic pain; dysentery & General & Kennedy 2006 \\
\hline Cardiospermum halicacabum L. & Climber & Whole plant & $\begin{array}{l}\text { Waist pain; joint pain; rheumatism and jaundice; tonic; } \\
\text { swellings; ear pain; body pain; paralysis; gastric problem; } \\
\text { throat infection; eczema; herpes; antibacterial; hydrocele; } \\
\text { fever; burns; ovulation enhancement; skin disease }\end{array}$ & $\begin{array}{l}\text { General; } \\
\text { women }\end{array}$ & Sharma and Mujundar 2003 \\
\hline $\begin{array}{l}\text { Deinbollia pinnata (Poir.) Schumach. and } \\
\text { Thonn. }\end{array}$ & $\begin{array}{l}\text { Shrub to } \\
\text { small tree }\end{array}$ & Leaves & Vegetable & General & Dansi et al. 2008 \\
\hline Dodonaea viscosa (L.) Jacq. & $\begin{array}{l}\text { Shrub to } \\
\text { small tree }\end{array}$ & Whole plant & $\begin{array}{l}\text { Bone fracture; fits; wound; rheumatism; bursitis; epilepsy; } \\
\text { insecticidal; wormicidal; headache }\end{array}$ & $\begin{array}{l}\text { General; } \\
\text { animals }\end{array}$ & $\begin{array}{l}\text { Rao et al. 2006, Ratnam and } \\
\text { Raju } 2008\end{array}$ \\
\hline Ganophyllum falcatum Blume & Tree & Bark & Snake Bite & General & Prasad et al. 2008 \\
\hline Harpullia arborea (Blanco) Radlk. & $\begin{array}{l}\text { Subcanopy } \\
\text { tree }\end{array}$ & Stem & To prevent leech & General & Udayan et al. 2005 \\
\hline Lepisanthes tetraphylla Radlk. & Tree & Fruit & - & General & Kennedy 2006 \\
\hline Sapindus emarginatus Vahl & Tree & $\begin{array}{l}\text { Endosperm, } \\
\text { bark, nut, } \\
\text { seed, fruit, } \\
\text { flower }\end{array}$ & $\begin{array}{l}\text { Tooth problems; centipede bite; surfactants; reduces hair } \\
\text { loss; intestinal worms; detergent; snakebite }\end{array}$ & General & Pradheeps and Poyyamoli 2013 \\
\hline Sapindus laurifolius Balb. ex DC. & Tree & Fruit, leaves & Anti-inflammatory; rheumatism; snakebite; insecticides & General & $\begin{array}{l}\text { Dey and De 2012, Kanthasamy } \\
\text { Kalaichelvi } 2016\end{array}$ \\
\hline Sapindus mukorossi Gaertn. & Tree & Fruit, seed & Abortifacient; rabies & General & Das et al. 2014 \\
\hline Sapindus saponaria L. & Tree & Fruit & - & - & Sharma et al. 2013 \\
\hline Sapindus trifoliatus L. & $\begin{array}{l}\text { Medium } \\
\text { sized tree }\end{array}$ & Fruit, root & Pruritus; edible; stomachache & General & Bhat et al. 2014 \\
\hline Schleichera oleosa (Lour.) Merr. & $\begin{array}{l}\text { Medium } \\
\text { sized tree }\end{array}$ & $\begin{array}{l}\text { Fruit, bark, } \\
\text { tuber, seed }\end{array}$ & $\begin{array}{l}\text { Asthma; purifies blood; chest pain; joint pain; skin } \\
\text { diseases; snakebite; edible }\end{array}$ & General & $\begin{array}{l}\text { Jain 2004, Dey et al. 2012, } \\
\text { Wagh and Jain } 2015\end{array}$ \\
\hline \multicolumn{6}{|l|}{ Sapotaceae } \\
\hline Isonandra lanceolata Wight & $\begin{array}{l}\text { Shrub to } \\
\text { small tree }\end{array}$ & $\begin{array}{l}\text { Leaves, } \\
\text { fruit, root } \\
\text { bark }\end{array}$ & - & General & Dey and De 2012 \\
\hline
\end{tabular}

Published 11 February 2019

http://dx.doi.org/10.32859/era.18.4.1-112 


\begin{tabular}{|c|c|c|c|c|c|}
\hline $\begin{array}{l}\text { Madhuca longifolia (J. Koenig ex L.) J.F. } \\
\text { Macbr. }\end{array}$ & Tree & $\begin{array}{l}\text { Nut, flower, } \\
\text { fruit, bark, } \\
\text { seed, } \\
\text { leaves, oil }\end{array}$ & $\begin{array}{l}\text { Skin diseases; snake bite; dysentery; diarrhoea; diabetes; } \\
\text { bone strengthening; pain reliving; inflammation; wound; } \\
\text { rheumatic pain; burns; psoriasis; scabies }\end{array}$ & General & $\begin{array}{l}\text { Gairola et al. 2013, Krishna et } \\
\text { al. 2014, Kosalge and Fursule } \\
\text { 2009, Savithramma et al. } 2014\end{array}$ \\
\hline Manilkara hexandra (Roxb.) Dubard & Tree & $\begin{array}{l}\text { Fruit, latex, } \\
\text { bark }\end{array}$ & Hip pains; toothache; fever & General & Padal et al. 2010 \\
\hline Manilkara zapota (L.) P. Royen & Tree & Bark & Wounds & General & Rao et al. 2006 \\
\hline Mimusops elengi $\mathrm{L}$. & Tree & $\begin{array}{l}\text { Bark, flower, } \\
\text { seed, root, } \\
\text { fruit, leaves, } \\
\text { seed oil } \\
\end{array}$ & $\begin{array}{l}\text { Toothache; uterus problems; gum; teeth diseases; liver } \\
\text { complaints; headache; asthma; biliousness; aphrodisiac; } \\
\text { diuretic; astringent to the bowels; gonorrhea; dysentery; } \\
\text { antipyretic; constipation; antiseptic }\end{array}$ & $\begin{array}{l}\text { General; } \\
\text { women }\end{array}$ & $\begin{array}{l}\text { Chopda and Mahajan 2009, } \\
\text { Ragupathy and Newmaster } \\
\text { 2009, Rao et al. } 2006\end{array}$ \\
\hline Palaquium ellipticum (Dalzell) Baill. & Tree & Oil, fruit & Illumination; ripe fruits for eating & General & Nazarudeen 2010 \\
\hline $\begin{array}{l}\text { Planchonella longipetiolata (King and } \\
\text { Prain) H.J. Lam }\end{array}$ & Tree & Leaves & Appetizer & General & Prasad et al. 2008 \\
\hline \multicolumn{6}{|l|}{ Saururaceae } \\
\hline Houttuynia cordata Thunb. & Herb & Whole plant & Stomachache and diarrhoea & General & Ruba and Mohan 2016 \\
\hline \multicolumn{6}{|l|}{ Saxifragaceae } \\
\hline Bergenia ciliata (Haw.) Sternb. & Herb & \begin{tabular}{|l|}
$\begin{array}{l}\text { Root, } \\
\text { rhizome }\end{array}$ \\
\end{tabular} & Wound healing & General & Kumar et al. 2007 \\
\hline Saxifraga hirculus L. & Herb & Whole plant & Cough; chest complaints; pulmonary disorders & General & Kayani et al. 2014 \\
\hline \multicolumn{6}{|l|}{ Schisandraceae } \\
\hline Schisandra chinensis (Turcz.) Baill. & Climber & - & Anti-viral & General & Zhang et al. 2013 \\
\hline \multicolumn{6}{|l|}{ Scrophulariaceae } \\
\hline Buddleja asiatica Lour. & Shrub & $\begin{array}{l}\text { Leaves, } \\
\text { flower, twig, } \\
\text { root }\end{array}$ & $\begin{array}{l}\text { Diarrhoea; Beverages fermentation; skin disease; asthma; } \\
\text { coughing With blood }\end{array}$ & General & $\begin{array}{l}\text { Ignacimuthu et al. 2008, Kayani } \\
\text { et al. } 2014\end{array}$ \\
\hline Kickxia elatine (L.) Dumort. & Herb & Stem & Haemostatic; wound healing; bleeding & General & Dhivya and Kalaichelvi 2015 \\
\hline Scrophularia auriculata L. & Herb & Leaves & For troubles linked to wounds & General & Parada et al. 2009 \\
\hline Verbascum chinense (L.) Santapau & Herb & Leaves & - & General & Kataria and Kaur 2013 \\
\hline \multicolumn{6}{|l|}{ Selaginellaceae } \\
\hline Bryodesma indicum (Milde) Soják & Herb & Leaves & Chronic wound or ulcer; multiple abscess & General & Hota and Padhi 2003 \\
\hline Selaginella bryopteris Baker & Herb & Whole plant & Infant disease; leucorrhoea & General & Sreeramulu et al. 2013 \\
\hline $\begin{array}{l}\text { Selaginella delicatula (Desv. ex Poir.) } \\
\text { Alston }\end{array}$ & Herb & Whole plant & Wounds & General & Latheef et al. 2014 \\
\hline Selaginella kraussiana (Kunze) A. Braun & Creeper & Aerial parts & Wounds & General & Das et al. 2013 \\
\hline $\begin{array}{l}\text { Selaginella repanda (Desv. ex Poir.) } \\
\text { Spring }\end{array}$ & Herb & Root & Fever & General & Padal et al. 2010 \\
\hline \multicolumn{6}{|l|}{ Simaroubaceae } \\
\hline Ailanthus excelsa Roxb. & Tree & $\begin{array}{l}\text { Leaves, } \\
\text { root, bark }\end{array}$ & $\begin{array}{l}\text { Snake bite and scorpion sting; typhoid; chronic bronchitis; } \\
\text { asthma and dyspeptic complaints; healing after child birth; } \\
\text { jaundice; piles; anorexia; tympany; Skin diseases; } \\
\text { jaundice; anthelmintic; antiasthmic Broncho constriction }\end{array}$ & General & $\begin{array}{l}\text { Alagesaboopathi } 2011 \text {, Padal et } \\
\text { al. 2014, Raghupathy and } \\
\text { Newmaster } 2009\end{array}$ \\
\hline Brucea mollis Wall. ex Kurz & Shrub & Fruit, seed & Malarial treatment & General & Jain 2004 \\
\hline Brucea javanica (L.) Merr. & Shrub & Seed & Malarial treatment & General & $\begin{array}{l}\text { Chenniappan and Kadarkarai } \\
2010\end{array}$ \\
\hline
\end{tabular}

Published 11 February 2019

http://dx.doi.org/10.32859/era.18.4.1-112 
Ethnobotany Research and Applications

\begin{tabular}{|c|c|c|c|c|c|}
\hline \multicolumn{6}{|l|}{ Smilacaceae } \\
\hline Smilax glabra Roxb. & Climber & - & Anti-cancer & General & Zhang et al. 2013 \\
\hline Smilax lanceifolia Roxb. & Climber & Rhizome & - & General & Sharma et al. 2013 \\
\hline Smilax ovalifolia Roxb. ex D. Don & Climber & Whole plant & $\begin{array}{l}\text { Jaundice; skin problems; toothache; urinary complain; } \\
\text { muscular sprain; stomach pain; rheumatic arthritis; } \\
\text { venereal diseases; infertility; as sexual stimulant; in } \\
\text { abnormal semen discharge; uterine diseases; sores; } \\
\text { dysentery; malaria; tuberculosis; leucorrhoea; wound } \\
\text { healing; to increase appetite; gastric disorders; antibiotic; } \\
\text { antifungal; antiseptic; blood purifier; skin diseases; } \\
\text { obestetric diseases; scar }\end{array}$ & $\begin{array}{l}\text { General; } \\
\text { women; } \\
\text { men }\end{array}$ & $\begin{array}{l}\text { Jain } 2004 \text {, Shah 2015, Srithi et } \\
\text { al. } 2009\end{array}$ \\
\hline Smilax perfoliata Lour. & Climber & Tuber & Abortion & General & Sreeramulu et al. 2013 \\
\hline Smilax zeylanica L. & Climber & $\begin{array}{l}\text { Root, } \\
\text { leaves, } \\
\text { rhizome }\end{array}$ & Venereal disorders; diabetes & General & Sreeramulu et al. 2013 \\
\hline \multicolumn{6}{|l|}{ Solanaceae } \\
\hline $\begin{array}{l}\text { Brugmansia suaveolens (Humb. and } \\
\text { Bonpl. ex Willd.) Bercht. and J. Presl }\end{array}$ & $\begin{array}{l}\text { Shrub to } \\
\text { small tree }\end{array}$ & Fruit & Migrane & General & Rajendran et al. 2000 \\
\hline Capsicum annuum L. & Herb & Whole plant & $\begin{array}{l}\text { Rheumatism; snake bite; dog bite; vegetable; asthma; } \\
\text { woophing cough; cold; gynaecological disorders; } \\
\text { vermicide }\end{array}$ & $\begin{array}{l}\text { General; } \\
\text { women }\end{array}$ & $\begin{array}{l}\text { Dansi et al. 2008, Dey and De } \\
\text { 2012, Kayani et al. } 2014\end{array}$ \\
\hline Cyphomandra betacea (Cav.) Sendtn. & $\begin{array}{l}\text { Shrub to } \\
\text { small tree }\end{array}$ & Fruit & Tuberculosis; diuretic; cough and cold & General & $\begin{array}{l}\text { Dhanabal et al. 2015, Rekka } \\
\text { and Kumar } 2014\end{array}$ \\
\hline Datura innoxia Mill. & Shrub & $\begin{array}{l}\text { Seed, root, } \\
\text { leaves, fruit }\end{array}$ & Leprosy; asthma & General & Kayani et al. 2014 \\
\hline Datura metel L. & Herb & Whole plant & $\begin{array}{l}\text { Snake bite; antimicrobial; epilepsy; rheumatic arthritis; } \\
\text { hysteria; painful mensturation; wounds; respiratory } \\
\text { troubles; muscle pain; antiplasmodic; cattle wounds; } \\
\text { asthma; dog bite; chicken pox; hair fall; abortifacient; } \\
\text { malaria; cerebral complications; skin diseases; insanity; } \\
\text { cough; swellings }\end{array}$ & $\begin{array}{l}\text { General; } \\
\text { women; } \\
\text { pregnant } \\
\text { women }\end{array}$ & Puravankara and Gopal 2012 \\
\hline Datura stramonium L. & Herb & $\begin{array}{l}\text { Leaves, } \\
\text { seed, fruit, } \\
\text { root, flower }\end{array}$ & $\begin{array}{l}\text { To reduce inflamed wound and sores; pain; epilepsy; } \\
\text { herpes; leprosy; skin diseases; gynaecological disorders; } \\
\text { asthma; bronchitis; expelling worms }\end{array}$ & General & $\begin{array}{l}\text { Kayani et al. 2014, Sharma et al. } \\
2013\end{array}$ \\
\hline Hyoscyamus albus $\mathrm{L}$. & Herb & Seed & For toothache & General & Parada et al. 2009 \\
\hline Hyoscyamus niger $\mathrm{L}$. & Herb & $\begin{array}{l}\text { Leaves, } \\
\text { seed, fruit }\end{array}$ & Asthma; cough & General & Kayani et al. 2014 \\
\hline Lycopersicon esculentum Mill. & Herb & Leaves & Headache; vegetable & General & $\begin{array}{l}\text { Dansi et al. 2008, Puravankara } \\
\text { and Gopal } 2012\end{array}$ \\
\hline Nicandra physalodes (L.) Gaertn. & Shrub & $\begin{array}{l}\text { Seed, } \\
\text { leaves }\end{array}$ & Analgesic; intestinal pain & General & Kanthasamy Kalaichelvi 2016 \\
\hline Nicotiana tabacum $\mathrm{L}$. & Herb & Leaves & Scabies; wound; snakebite; tooth decay; tooth ache & General & Samy et al. 2008 \\
\hline Physalis angulata $\mathrm{L}$. & Herb & Fruit, leaves & Anti microbial; antifungal; vegetable & General & Dansi et al. 2008 \\
\hline
\end{tabular}

Published 11 February 2019

http://dx.doi.org/10.32859/era.18.4.1-112 


\begin{tabular}{|c|c|c|c|c|c|}
\hline Physalis minima $\mathrm{L}$. & Herb & $\begin{array}{l}\text { Fruit, } \\
\text { leaves, seed }\end{array}$ & $\begin{array}{l}\text { Leucorrhea; dropsy; constipation; enlargement of spleen; } \\
\text { colic complaints; jaundice; constipation; laxative; } \\
\text { abdominal troubles; gonorrhea }\end{array}$ & $\begin{array}{l}\text { General; } \\
\text { men; } \\
\text { women }\end{array}$ & $\begin{array}{l}\text { Bhagat et al. 2016, Kanthasamy } \\
\text { Kalaichelvi } 2016\end{array}$ \\
\hline Solanum aethiopicum $\mathrm{L}$. & Shrub & Leaves & Vegetable & General & Dansi et al. 2008 \\
\hline Solanum americanum Mill. & Herb & Whole plant & $\begin{array}{l}\text { Diabetes; lactagogue; antimicrobial; eczema; enhances } \\
\text { fertility; fever; diarrhoea; malaria; cough; insecticidal; } \\
\text { wormicidal; intestinal ulcers; oral health care; mouth sores; } \\
\text { post natal complaints; improves vision; stomachache; } \\
\text { ulcer; wound; blood spitting; fertility problems }\end{array}$ & General & $\begin{array}{l}\text { Bhagat et al. 2016, Jain 2004, } \\
\text { Kayani et al. 2014, Ramya 2008, } \\
\text { Shanmugam et al. } 2012\end{array}$ \\
\hline Solanum anguivi Lam. & Shrub & $\begin{array}{l}\text { Fruit, } \\
\text { leaves, root, } \\
\text { seed }\end{array}$ & $\begin{array}{l}\text { Leucorrhea; skin cuts; scorpion sting; cold; cough; fever; } \\
\text { intestinal worms; toothache; tooth decay; destroys round } \\
\text { worms }\end{array}$ & General & $\begin{array}{l}\text { Bhagat et al. 2016, Ragupathy } \\
\text { and Newmaster } 2009\end{array}$ \\
\hline Solanum capsicoides All. & $\begin{array}{l}\text { Herb/ } \\
\text { undershrub }\end{array}$ & Seed & Snakebite & General & Dey and De 2012 \\
\hline $\begin{array}{l}\text { Solanum dasyphyllum Schumach. and } \\
\text { Thonn. }\end{array}$ & Herb & Leaves & Vegetable & General & Dansi et al. 2008 \\
\hline Solanum erianthum D. Don & Shrub & $\begin{array}{l}\text { Leaves, } \\
\text { root, fruit }\end{array}$ & Dysentery; fever; diarrhoea; indigestion & General & Kanthasamy Kalaichelvi 2016 \\
\hline Solanum ferox $\mathrm{L}$. & Herb & Root, fruit & Itching of skin; nailside infection & General & Kshirsagar and Singh 2001 \\
\hline Solanum indicum L. & Shrub & $\begin{array}{l}\text { Root, fruit, } \\
\text { seed, leaves }\end{array}$ & $\begin{array}{l}\text { Snakebite; against blood vomiting; mouth ulcer; toothache; } \\
\text { anthelmintic; breverages fermentation }\end{array}$ & General & $\begin{array}{l}\text { Manikandan 2005, Ruba and } \\
\text { Mohan } 2016\end{array}$ \\
\hline Solanum Iyratum Thunb. & Climber & - & Anti-tumour & General & Zhang et al. 2013 \\
\hline Solanum macrocarpon $\mathrm{L}$. & Herb & Leaves & Vegetable & General & Dansi et al. 2008 \\
\hline Solanum melongena $\mathrm{L}$. & Shrub & - & Asthma & General & Rajagopal et al. 2016 \\
\hline Solanum rudepannum Dunal & Shrub & Fruit, leaves & Cough; cold; anti-inflammatory; fever & General & Kanthasamy Kalaichelvi 2016 \\
\hline Solanum scabrum Mill. & Shrub & Leaves & Vegetable & General & Dansi et al. 2008 \\
\hline Solanum sisymbriifolium Lam. & Herb & Root & For toothache & General & Puravankara and Gopal 2012 \\
\hline Solanum surattense Burm. f. & Shrub & $\begin{array}{l}\text { Seed, fruit, } \\
\text { root, leaves }\end{array}$ & $\begin{array}{l}\text { Leprosy; toothache; abortion; constipation; antiasthmatic; } \\
\text { vomiting; gastrointestinal disorders; menstural problems }\end{array}$ & $\begin{array}{l}\text { General; } \\
\text { women; } \\
\text { pregnant } \\
\text { women }\end{array}$ & $\begin{array}{l}\text { Hebbar et al. 2004, Kumar et al. } \\
2016\end{array}$ \\
\hline Solanum torvum Sw. & Shrub & $\begin{array}{l}\text { Root, fruit, } \\
\text { seed, leaves }\end{array}$ & $\begin{array}{l}\text { Snake bite; skin injuries; wounds; abortifacient; } \\
\text { antibacterial; edible; tuberculosis }\end{array}$ & $\begin{array}{l}\text { General; } \\
\text { pregnant } \\
\text { women }\end{array}$ & $\begin{array}{l}\text { Dey and De 2012, Samy et al. } \\
2008\end{array}$ \\
\hline Solanum trilobatum $\mathrm{L}$. & Shrub & Whole plant & Bronchitis; asthma; cold; cough & General & Padal et al. 2010, Ramya 2008 \\
\hline Solanum vagum Heyn. ex Nees & $\begin{array}{l}\text { Shrub/under } \\
\text { shrub }\end{array}$ & Leaves, root & To treat dry skin & General & Ayyanar and Ignacimuthu 2005 \\
\hline Solanum verbascifolium $\mathrm{L}$. & Shrub & Root, bark & Stomach pain; foe easy pregnancy and delivery & $\begin{array}{l}\text { General; } \\
\text { women }\end{array}$ & Puravankara and Gopal 2012 \\
\hline Solanum violaceum Ortega & Shrub & Fruit & Asthma; cardiac disorders; oral health care & General & Hebbar et al. 2004 \\
\hline Solanum virginianum $\mathrm{L}$. & Subshrub & Whole plant & $\begin{array}{l}\text { Cough; asthma; bronchitis and chest affections; sore } \\
\text { throat; diuretic; toothache; swelling; abortifacient; skin } \\
\text { diseases; laxative; rheumatism; diabetes; ulcers; heart } \\
\text { diseases }\end{array}$ & $\begin{array}{l}\text { General; } \\
\text { pregnant } \\
\text { women }\end{array}$ & Wagh and Jain 2015 \\
\hline Syringa pubescens Turcz. & Shrub & Fruit & Edible & General & RamaChandran 2007 \\
\hline
\end{tabular}

Published 11 February 2019

http://dx.doi.org/10.32859/era.18.4.1-112 


\begin{tabular}{|c|c|c|c|c|c|}
\hline Withania somnifera (L.) Dunal & Shrub & Whole plant & $\begin{array}{l}\text { Amenorrhegia; anthelmintic; rheumatic swelling; fever; } \\
\text { asthma; bronchitis; sexual potency; debility; aphrodisiac; } \\
\text { leucorrhea; ulcers; sex stimulant; skin disease; snakebite; } \\
\text { enhances sperm count; antiinflammatory; insecticidal; } \\
\text { wormicidal; abcesses; stomachache }\end{array}$ & $\begin{array}{l}\text { General; } \\
\text { women; } \\
\text { men }\end{array}$ & $\begin{array}{l}\text { Dey and De 2012, Mahishi et al } \\
\text { 2005, Naidu et al. } 2013\end{array}$ \\
\hline \multicolumn{6}{|l|}{ Sphagnaceae } \\
\hline Sphagnum sericeum Müll. Hal. & Shrub & - & $\begin{array}{l}\text { Used to dressing wounds; with anti-microbial properties for } \\
\text { skin ailments (insects bites; scabies; acne); haemorrhoids } \\
\text { and to treat eye diseases }\end{array}$ & General & Chandra et al. 2016 \\
\hline Sphagnum teres (Schimp.) Ångström & Moss & - & Used to treat eye diseases & General & Chandra et al. 2016 \\
\hline \multicolumn{6}{|l|}{ Sphenocleaceae } \\
\hline Sphenoclea zeylanica Gaertn. & Herb & Leaves & Vegetable & General & Dansi et al. 2008 \\
\hline \multicolumn{6}{|l|}{ Staphyleaceae } \\
\hline Staphylea emodi Wall. & Shrub & Whole plant & - & General & Dey and De 2012 \\
\hline \multicolumn{6}{|l|}{ Stemonaceae } \\
\hline Stemona tuberosa Lour. & Climber & $\begin{array}{l}\text { Root, tuber, } \\
\text { leaves }\end{array}$ & Dysentery; Fever; stomachache & General & Rao et al. 2006, Rao et al. 2011 \\
\hline \multicolumn{6}{|l|}{ Styracaceae } \\
\hline Styrax benzoin Dryand. & Tree & Sap & - & General & Kennedy 2006 \\
\hline \multicolumn{6}{|l|}{ Symplocaceae } \\
\hline Symplocos racemosa Roxb. & Tree & Root & Chest and back sprains & General & Natarajan and Paulsen 2000 \\
\hline \multicolumn{6}{|l|}{ Talinaceae } \\
\hline Talinum fruticosum (L.) Juss. & Shrub & Leaves & Vegetable & General & Dansi et al. 2008 \\
\hline \multicolumn{6}{|l|}{ Tamaricaceae } \\
\hline Tamarix gallica L. & Shrub & Whole plant & - & General & Chopda and Mahajan 2009 \\
\hline \multicolumn{6}{|l|}{ Taxaceae } \\
\hline Taxus baccata L. & Tree & Leaves & Antispasmodic; epilepsy & General & Sharma et al. 2013 \\
\hline \multicolumn{6}{|l|}{ Tetramelaceae } \\
\hline Tetrameles nudiflora R. Br. & Tree & Tender tips & Insect sting & General & Prasad et al. 2008 \\
\hline \multicolumn{6}{|l|}{ Theaceae } \\
\hline Camellia sinensis (L.) Kuntze & Tree & Root, flower & Coughing up With blood & General & Kayani et al. 2014 \\
\hline Schima wallichii Choisy & Tree & $\begin{array}{l}\text { Bark, } \\
\text { leaves, fruit }\end{array}$ & Cuts and wounds & General & Kumar et al. 2007 \\
\hline \multicolumn{6}{|l|}{ Thelypteridaceae } \\
\hline Ampelopteris prolifera (Retz.) Copel. & Fern & $\begin{array}{l}\text { Leaves, } \\
\text { rhizome }\end{array}$ & Skin diseases & General & Bharti and Pravesh 2012 \\
\hline Christella parasitica (L.) Holttum & Herb & Leaves & Snakebite & General & Dey and De 2012 \\
\hline Sphaerostephanos unitus (L.) Holttum & Frond & Whole plant & Scorpion sting & General & Sreeramulu et al. 2013 \\
\hline \multicolumn{6}{|l|}{ Thuidiaceae } \\
\hline $\begin{array}{l}\text { Haplocladium microphyllum (Hedw.) } \\
\text { Broth. }\end{array}$ & Moss & - & $\begin{array}{l}\text { Used to treat cystisis; bronchitis; tonsillitis pneumonia and } \\
\text { fever }\end{array}$ & General & Chandra et al. 2016 \\
\hline \multicolumn{6}{|l|}{ Thymelaeaceae } \\
\hline Aquilaria agallocha Roxb. & Tree & Latex & Infertility in cow; twitching & Veterinary & Chopda and Mahajan 2009 \\
\hline
\end{tabular}

Published 11 February 2019

http://dx.doi.org/10.32859/era.18.4.1-112 


\begin{tabular}{|c|c|c|c|c|c|}
\hline Typha angustifolia L. & Herb & Root & $\begin{array}{l}\text { Indigestion; diarrhoea; dysentery; fever; headache; cough; } \\
\text { abdomen pain }\end{array}$ & General & Chander et al. 2015 \\
\hline Typha domingensis Pers. & Herb & Aerial parts & Urinary calculi; dysuria; inflammation & General & $\begin{array}{l}\text { Prashanth Kumar and } \\
\text { Shiddamallayya } 2015\end{array}$ \\
\hline Typha elephantina Roxb. & Shrub & Fruit & - & General & Chopda and Mahajan 2009 \\
\hline \multicolumn{6}{|l|}{ Ulmaceae } \\
\hline Holoptelea integrifolia Planch. & Tree & $\begin{array}{l}\text { Leaves, } \\
\text { bark, twig, } \\
\text { root, latex, } \\
\text { flower }\end{array}$ & $\begin{array}{l}\text { Piles; wounds; skin diseases; paralysis; swelling; scorpion } \\
\text { sting; malaria; herpes }\end{array}$ & General & $\begin{array}{l}\text { Murty and Venkaiah 2010, Rao } \\
\text { et al. } 2006\end{array}$ \\
\hline \multicolumn{6}{|l|}{ Urticaceae } \\
\hline Debregeasia longifolia (Burm.f.) Wedd. & $\begin{array}{l}\text { Shrub to } \\
\text { small tree }\end{array}$ & Fruit & Edible & General & Nazarudeen 2010 \\
\hline Dendrocnide sinuata (Blume) Chew & Shrub & Root & - & - & Kataria and Kaur 2013 \\
\hline Girardinia diversifolia (Link) Friis & Herb & Leaves, root & Herpes; pruritus; scabies; scabby & General & $\begin{array}{l}\text { Bhat et al. 2014, Jagtap et al. } \\
2009\end{array}$ \\
\hline Laportea aestuans (L.) Chew & Herb & Fruit, leaves & Mental depression symptoms; vegetable & General & Nanjunda 2010 \\
\hline Parietaria officinalis L. & Herb & Aerial parts & For stones in the kidney & General & Parada et al. 2009 \\
\hline Pellionia heyneana Wedd. & Herb & Leaves & Liver ailments; immunity & $\begin{array}{l}\text { General; } \\
\text { children }\end{array}$ & Vilash et al. 2016 \\
\hline Pouzolzia auriculata Wight & Shrub & Whole plant & Leucoderma; itching & General & Sripathi and Sankari 2010 \\
\hline Pouzolzia bennettiana Wight & Shrub & Leaves & Stomach disorder & General & Ruba and Mohan 2016 \\
\hline Pouzolzia wightii Benn. and $\mathrm{Br}$. & Herb & Leaves & Blood clotting; itching & General & Rajendran et al. 2002 \\
\hline Pouzolzia zeylanica (L.) Benn. & Herb & Aerial parts & Bone fracture; kidney stone; galactagogue & General & Ratnam and Raju 2008 \\
\hline Urtica dioica $\mathrm{L}$. & Herb & Root & Snake bite & General & Hiremath and Taranath 2010 \\
\hline \multicolumn{6}{|l|}{ Verbenaceae } \\
\hline Aloysia citriodora Palau & Shrub & - & Digestion; stomach ache & General & Parada et al. 2009 \\
\hline Lantana camara L. & Shrub & Whole plant & $\begin{array}{l}\text { Cold; fever; vomiting; wound; antibacterial; antidiabetic; } \\
\text { cuts; rheumatism; ulcers; tetanus; malaria; cancer; } \\
\text { asthma; ulcer; swelling; high blood pressure; bilious; fever; } \\
\text { skin itches; menstrual disorders; piles; digestion; burns; } \\
\text { antidiarrhoel; skin inflammations }\end{array}$ & Children & $\begin{array}{l}\text { Ayyanar and Ignacimuthu 2005, } \\
\text { Dey and De 2012, Umapriya et } \\
\text { al. } 2011\end{array}$ \\
\hline Lantana indica Roxb. & Shrub & Whole plant & Gas troubles; snake bite; fits; scorpion sting; headache & $\begin{array}{l}\text { Children; } \\
\text { general }\end{array}$ & $\begin{array}{l}\text { Dey and De 2012, Sharma et al. } \\
2013\end{array}$ \\
\hline Lantana montevidensis (Spreng.) Briq. & $\begin{array}{l}\text { Shrub or } \\
\text { subshrub }\end{array}$ & Fruit & Edible & General & Rekka and Kumar 2014 \\
\hline Lippia chevalieri Moldenke & Herb & Leaves & Malarial treatment & General & $\begin{array}{l}\text { Chenniappan and Kadarkarai } \\
2010\end{array}$ \\
\hline Lippia javanica (Burm. f.) Spreng. & Herb & Leaves & Indigestion & General & Ignacimuthu et al. 2008 \\
\hline Lippia multiflora Moldenke & Herb & Leaves & Vegetable & General & Dansi et al. 2008 \\
\hline Phyla nodiflora (L.) Greene & Herb & Whole plant & $\begin{array}{l}\text { Diabetes; antiseptic; painful joints; malarial fevers; asthma; } \\
\text { pulmonary infections; fever; antibacterial; jaundice; } \\
\text { promotes sexual desire; dandruff; diarrhoea; stomachache }\end{array}$ & $\begin{array}{l}\text { General; } \\
\text { children }\end{array}$ & $\begin{array}{l}\text { Basha et al. 2011, Lingaraju et } \\
\text { al. } 2013\end{array}$ \\
\hline Priva cordifolia (L.f.) Druce & Herb & Whole plant & Wounds & General & Ignacimuthu et al. 2008 \\
\hline
\end{tabular}

Published 11 February 2019

http://dx.doi.org/10.32859/era.18.4.1-112 


\begin{tabular}{|c|c|c|c|c|c|}
\hline Stachytarpheta indica (L.) Vahl & Shrub & Root, leaves & $\begin{array}{l}\text { Fever; cold; asthma; dysentery; vegetable; diarrhoea; } \\
\text { intestinal worms }\end{array}$ & General & $\begin{array}{l}\text { Dansi et al. 2008, Shanmugam } \\
\text { et al. } 2012\end{array}$ \\
\hline Stachytarpheta jamaicensis (L.) Vahl & Shrub & Leaves, root & $\begin{array}{l}\text { Dysentery; cuts; wounds; intestinal worms; ulcers; } \\
\text { stomach ailments }\end{array}$ & General & Jayanthi et al. 2012 \\
\hline \multicolumn{6}{|l|}{ Viburnaceae } \\
\hline Sambucus javanica Blume & Small tree & Aerial parts & $\begin{array}{l}\text { Treating fragile bone or osteoarthritis; obstetric diseases } \\
\text { or body pain }\end{array}$ & General & Srithi et al. 2009 \\
\hline Sambucus nigra L. & Shrub & Flowers & $\begin{array}{l}\text { Earache; headache; bellyache; sore throat; cough; wound } \\
\text { infection; clear nose; clean eyes }\end{array}$ & General & Parada et al. 2009 \\
\hline \multicolumn{6}{|c|}{ ( } \\
\hline Hybanthus enneaspermus (L.) F. Muell. & Herb & Whole plant & $\begin{array}{l}\text { Urinary problems; reduce body heat; diuretic; urinary } \\
\text { troubles; bowel complaints; treats relaxation of nerves; } \\
\text { vegetable; joint pains; nerve stimulant }\end{array}$ & $\begin{array}{l}\text { General; } \\
\text { children }\end{array}$ & Shanmugam et al. 2012 \\
\hline Hybanthus linearifolius (Vahl) Urb. & Herb & Whole plant & $\begin{array}{l}\text { Increases male sterility; urinary tract infections; to avoid } \\
\text { any urino-genital infection during pregnancy; conjunctivitis; } \\
\text { hair shampoo }\end{array}$ & $\begin{array}{l}\text { General; } \\
\text { pregnant } \\
\text { women; } \\
\text { men }\end{array}$ & Pushpangadan and Atal 1984 \\
\hline Viola betonicifolia Sm. & Herb & $\begin{array}{l}\text { Leaves, } \\
\text { flower, root, } \\
\text { fruit }\end{array}$ & Lung troubles; cough; colds & General & Kayani et al. 2014 \\
\hline Viola canescens Wall. & Undershrub & Whole plant & Sore throat; cough & General & Kayani et al. 2014 \\
\hline Viola pilosa Blume & Herb & Root & Bronchitis; asthma; cough; cold & General & Kayani et al. 2014 \\
\hline \multicolumn{6}{|l|}{ Vitaceae } \\
\hline Ampelocissus indica (L.) Planch. & Climber & Root & Treatments of herpes & General & $\begin{array}{l}\text { Bhandary and Chandrashekar } \\
2011\end{array}$ \\
\hline Ampelocissus latifolia (Roxb.) Planch. & Climber & Whole plant & $\begin{array}{l}\text { Anorexia; dyspepsia and indigestion; bone fracture; } \\
\text { tuberculosis }\end{array}$ & General & Swarnkar and Katewa 2008 \\
\hline $\begin{array}{l}\text { Ampelocissus tomentosa (B. Heyne and } \\
\text { Roth) Planch. }\end{array}$ & Climber & Leaves, root & Bone fracture; Insect bite & General & Sreeramulu et al. 2013 \\
\hline Cayratia pedata (Lam.) Gagnep. & Climber & $\begin{array}{l}\text { Leaves, } \\
\text { bark, fruit, } \\
\text { stem }\end{array}$ & $\begin{array}{l}\text { Venereal diseases; non medicinal commercial purposes; } \\
\text { gastric complaints }\end{array}$ & General & $\begin{array}{l}\text { Ayyanar and Ignacimuthu 2005, } \\
\text { Sreeramulu et al. } 2013\end{array}$ \\
\hline Cayratia trifolia (L.) Domin & Climber & Whole plant & $\begin{array}{l}\text { To check sugar level of blood; snake bite; antiviral; } \\
\text { antibacterial; antiprotozoal; anticancer; diuretic; eczema; } \\
\text { wound; skin disease; depressant; anaemia }\end{array}$ & General & $\begin{array}{l}\text { Jain et al. 2008, Jain et al. } 2009 \text {, } \\
\text { Perumal et al. } 2015\end{array}$ \\
\hline Cissus adnata Roxb. & Climber & Whole plant & $\begin{array}{l}\text { Rheumatoid arthritis; snake bite; lice eradication; Skin } \\
\text { disease; bone fracture; wound }\end{array}$ & General & $\begin{array}{l}\text { Rao et al. 2006, Padma et al. } \\
2016\end{array}$ \\
\hline Cissus elongata Roxb. & Climber & Root & Inter trigo & General & Bhat et al. 2014 \\
\hline Cissus heyneana Planch. & Climber & Tuber & Jaundice & General & Ignacimuthu et al. 2008 \\
\hline Cissus javana DC. & Climber & Whole plant & Ringworm & General & Bhat et al. 2014 \\
\hline Cissus quadrangularis $\mathrm{L}$. & Climber & Whole plant & $\begin{array}{l}\text { Rheumatism; stomachache; indigestion and constipation; } \\
\text { wound; bone fracture; swellings; dyspepsia; indigestion; } \\
\text { constipation; earache; during pregnancy; reguralize } \\
\text { mensturation; piles; dog ite; sprains; to stimulate hunger }\end{array}$ & $\begin{array}{l}\text { women; } \\
\text { General; } \\
\text { pregnant } \\
\text { women; } \\
\text { goat }\end{array}$ & $\begin{array}{l}\text { Ayyanar 2013, Sharma et al. } \\
2013, \text { Ignacimuthu et al. 2008, } \\
\text { Kanthasamy Kalaichelvi 2016, } \\
\text { Sreeramulu et al. 2013, Rao et } \\
\text { al. } 2006\end{array}$ \\
\hline
\end{tabular}

Published 11 February 2019

http://dx.doi.org/10.32859/era.18.4.1-112 
Ethnobotany Research and Applications

\begin{tabular}{|c|c|c|c|c|c|}
\hline Cissus repanda (Wight and Arn.) Vahl & Climber & Leaves, root & Skin diseases; itch & General & Kanthasamy Kalaichelvi 2016 \\
\hline Cissus vitiginea L. & Climber & Whole plant & $\begin{array}{l}\text { Cooling agent; anti-inflammatory; sexual diseases; } \\
\text { increases potency; leucorrhoea; menorrhagia }\end{array}$ & $\begin{array}{l}\text { General; } \\
\text { men; } \\
\text { women }\end{array}$ & Savithramma et al. 2014 \\
\hline $\begin{array}{l}\text { Cyphostemma adenocaule (Steud. ex A. } \\
\text { Rich.) Desc. ex Wild and R.B. Drumm. }\end{array}$ & Climber & Leaves & Vegetable & General & Dansi et al. 2008 \\
\hline $\begin{array}{l}\text { Cyphostemma auriculatum (Roxb.) P. } \\
\text { Singh and B.V. Shetty }\end{array}$ & Climber & Whole plant & Wound healing & General & Kosalge and Fursule 2009 \\
\hline Cyphostemma setosum (Roxb.) Alston & Climber & $\begin{array}{l}\text { Leaves, } \\
\text { whole plant }\end{array}$ & Intestinal worms; rheumatism; dysentery; menorrhegia & $\begin{array}{l}\text { General; } \\
\text { women }\end{array}$ & $\begin{array}{l}\text { Shanmugam et al. 2012, } \\
\text { Sreeramulu et al. } 2013\end{array}$ \\
\hline $\begin{array}{l}\text { Cyphostemma trilobata (Lam.) M.R. } \\
\text { Almeida }\end{array}$ & Climber & Leaves, root & Rheumatism & General & Ayyanar and Ignacimuthu 2005 \\
\hline Leea aequata $\mathrm{L}$. & Shrub & Root & - & General & Chopda and Mahajan 2009 \\
\hline Leea asiatica (L.) Ridsdale & Shrub & $\begin{array}{l}\text { Bulb, root, } \\
\text { stem }\end{array}$ & Joint disease; insect bite; boils; blisters; wound & General & $\begin{array}{l}\text { Prasad et al. } 2008, \text { Sreeramulu } \\
\text { et al. } 2013\end{array}$ \\
\hline Leea compactiflora Kurz & Shrub & Flower, fruit & Snakebite & General & Dey and De 2012 \\
\hline Leea indica (Burm. f.) Merr. & $\begin{array}{l}\text { Shrub to } \\
\text { small tree }\end{array}$ & Aerial parts & $\begin{array}{l}\text { Obestric diseases; antibacterial; body pain; liver } \\
\text { enlargement; wound }\end{array}$ & General & $\begin{array}{l}\text { Bhagat et al. 2016, Srithi et al. } \\
2009\end{array}$ \\
\hline Leea macrophylla Roxb. ex Hornem. & Shrub & Bulb & Skin disorder & General & Wagh and Jain 2015 \\
\hline Vitis heyneana Roem. and Schult. & $\begin{array}{l}\text { Climbing } \\
\text { vine }\end{array}$ & Tuber & Fever; Sciatica & General & Rao et al. 2011 \\
\hline Vitis vinifera L. & $\begin{array}{l}\text { Climbing } \\
\text { vine }\end{array}$ & Fruit & Itching due to impure blood & General & Bhat et al. 2014 \\
\hline \multicolumn{6}{|l|}{ Xanthorrhoeaceae } \\
\hline Aloe vera (L.) Burm. f. & Herb & Whole plant & $\begin{array}{l}\text { Gastric problems; indigestion; intestinal ulcer and } \\
\text { vomiting; inflammation; to prevent abortion; wound; skin } \\
\text { disease; diarrhoea; piles; insect bite; hair problems; } \\
\text { insecticidal; wormicidal; gynaecological disorders; } \\
\text { jaundice; menstural disorders; swellings; ulcer; malaria; } \\
\text { liver diseases; diabetes; rheumatism }\end{array}$ & General & $\begin{array}{l}\text { Bhardwaj et al. 2011, Bhat et al. } \\
\text { 2014, Das et al. 2014, Jain } \\
\text { 2004, Ignacimuthu et al. 2008, } \\
\text { Parada et al. } 2009\end{array}$ \\
\hline Hemerocallis fulva (L.) L. & Herb & Rhizome & Fire burn skin; during hotness of chest and stomach & General & Namsa et al. 2009 \\
\hline Hemerocallis lilioasphodelus L. & Herb & Leaves & Treating cough; tonic; post-labor tonic & General & Srithi et al. 2009 \\
\hline \multicolumn{6}{|l|}{ Zingiberaceae } \\
\hline Alpinia calcarata (Haw.) Roscoe & Herb & Rhizome & Fungal infections on skin; asthma; growth & General & Pushpangadan and Atal 1984 \\
\hline Alpinia galanga (L.) Willd. & Herb & Rhizome & $\begin{array}{l}\text { inflammation and skin allergy caused by insect bites or } \\
\text { microbes; hypnotic patients; rheumatism;aphrodisiac }\end{array}$ & General & Namsa et al. 2009 \\
\hline Alpinia officinarum Hance & Herb & - & Anti-fungal & General & Zhang et al. 2013 \\
\hline Curcuma amada Roxb. & Herb & $\begin{array}{l}\text { Tuber, } \\
\text { flower, } \\
\text { rhizome }\end{array}$ & $\begin{array}{l}\text { Indigestion; anti-inflammatory; antioxidant; anti-mutagenic; } \\
\text { anti-cancer; insecticidal; wormicidal; diuretic; hemostatic; } \\
\text { headache }\end{array}$ & General & $\begin{array}{l}\text { Bhardwaj et al. 2011, Dhal et al. } \\
2011\end{array}$ \\
\hline Curcuma angustifolia Roxb. & Herb & $\begin{array}{l}\text { Rhizome, } \\
\text { tuber }\end{array}$ & $\begin{array}{l}\text { Promotes lactation; chronic diseases; fevers; internal body } \\
\text { irritations; worms; anaemia; aphrodisiac }\end{array}$ & $\begin{array}{l}\text { Children; } \\
\text { lactating } \\
\text { mothers }\end{array}$ & $\begin{array}{l}\text { Malviya et al. 2011, Sharma } \\
2009\end{array}$ \\
\hline Curcuma aromatica Salisb. & Herb & $\begin{array}{l}\text { Rhizome, } \\
\text { tuber, root }\end{array}$ & $\begin{array}{l}\text { Diarrrhoea; dysentery; skin diseases; insecticidal; } \\
\text { wormicidal; pimples; anticancer; antiseptic; fever }\end{array}$ & General & $\begin{array}{l}\text { Bosco and Arumugam 2012, } \\
\text { Dhal et al. } 2011\end{array}$ \\
\hline
\end{tabular}

Published 11 February 2019

http://dx.doi.org/10.32859/era.18.4.1-112 


\begin{tabular}{|c|c|c|c|c|c|}
\hline Curcuma caesia Roxb. & Herb & Rhizome & Scorpion and snake bite; inscet bite; pimples & General & $\begin{array}{l}\text { Namsa et al. 2009, Sharma et } \\
\text { al. } 2013\end{array}$ \\
\hline Curcuma inodora Blatt. & Herb & Rhizome & Cough and cold & General & Raju et al. 2014 \\
\hline Curcuma longa L. & Herb & Whole plant & $\begin{array}{l}\text { Cold; inflammations; skin disease; tooth ache; antiseptic; } \\
\text { cuts; wounds; eye disease; cough; asthma; gonorrhea; } \\
\text { urinary disease; diabetes; dysentery; body ache; jaundice; } \\
\text { menstural problems; gynaecological disorders; eczema; } \\
\text { bed sore; antisnake venom; postpartum health; increases } \\
\text { lactation; malaria; chronic liver diseases }\end{array}$ & General & $\begin{array}{l}\text { Asha and Pushpangadan 2002, } \\
\text { Chenniappan and Kadarkarai } \\
\text { 2010, Chithra et al. 2016, } \\
\text { Manikandan 2005, Muralidhara } \\
\text { Rao and Pullaiah 2007, Samy et } \\
\text { al. } 2008\end{array}$ \\
\hline Curcuma petiolata Roxb. & Herb & Tuber & Wounds & General & Prasad et al. 2008 \\
\hline Curcuma pseudomontana J. Graham & Herb & $\begin{array}{l}\text { Rhizome, } \\
\text { tuber }\end{array}$ & $\begin{array}{l}\text { Pneumonia; restorative diet and nervous tonic; wound; } \\
\text { stomachache; tuberculosis; cardiac disorders; muscle } \\
\text { stress }\end{array}$ & General & $\begin{array}{l}\text { Udayan et al. 2005, Wagh and } \\
\text { Jain } 2015\end{array}$ \\
\hline Curcuma zanthorrhiza Roxb. & Herb & Rhizome & Antimicrobial & General & Mary et al. 2012 \\
\hline Curcuma zedoaria (Christm.) Roscoe & Herb & $\begin{array}{l}\text { Rhizome, } \\
\text { tuber }\end{array}$ & $\begin{array}{l}\text { Abdominal cramps; amenorrhea-abdominal pain and } \\
\text { rheumatic pain; dyspepsia; colic; vomiting; cough and } \\
\text { menstrual disorders; bone fracture; diarrhoea; antibacterial }\end{array}$ & General & $\begin{array}{l}\text { Dhal et al. 2011, Zang et al. } \\
2013\end{array}$ \\
\hline Elettaria cardamomum (L.) Maton & Herb & Fruit & Stomache ache & General & Ramya 2008 \\
\hline Globba marantina L. & Herb & $\begin{array}{l}\text { Tuber, } \\
\text { rhizome, } \\
\text { root }\end{array}$ & Leucoderma; white spots; diabetes; lactogogue & General & Ram et al. 2004 \\
\hline Globba orixensis Roxb. & Herb & Rhizome & Scorpion stings & General & Das and Misra 1988 \\
\hline Globba racemosa Sm. & Herb & Fruit & Heart pain; Stomach pain & General & Rao et al. 2011 \\
\hline $\begin{array}{l}\text { Hedychium coccineum Buch. -Ham. ex } \\
\text { Sm. }\end{array}$ & Herb & Rhizome & Sexual debility; antiseptic; foot swelling; rheumatism & General & Namsa et al. 2009 \\
\hline Hedychium flavescens Carey ex Roscoe & Herb & $\begin{array}{l}\text { Rhizome, } \\
\text { leaves }\end{array}$ & Diarrhoea & General & Udayan et al. 2005 \\
\hline Hedychium spicatum Sm. & Herb & $\begin{array}{l}\text { Rhizome, } \\
\text { root }\end{array}$ & - & - & Chopda and Mahajan 2009 \\
\hline Kaempferia galanga L. & Herb & - & Postpartum health & General & Chithra et al. 2016 \\
\hline Kaempferia rotunda $\mathrm{L}$. & Herb & Whole plant & - & General & Chopda and Mahajan 2009 \\
\hline Zingiber cernuum Dalzell & Herb & Rhizome & Stomachache & General & Kshirsagar and Singh 2001 \\
\hline Zingiber officinale Roscoe & Herb & $\begin{array}{l}\text { Rhizome, } \\
\text { tuber, root }\end{array}$ & $\begin{array}{l}\text { Contraceptive; abortion; asthma; after delivery tonic; } \\
\text { antidiarrhoeal; cough; cold; gastrointestinal disorders; } \\
\text { headache; indigestion; labour pain; rheumatism; stomach } \\
\text { problems; liver pain; bronchitis; sore throat; earache; } \\
\text { intestinal gas }\end{array}$ & $\begin{array}{l}\text { General; } \\
\text { pregnant } \\
\text { women }\end{array}$ & $\begin{array}{l}\text { Jain et al. 2004, Ignacimuthu et } \\
\text { al. 2008, Namsa et al. } 2009\end{array}$ \\
\hline Zingiber roseum (Roxb.) Roscoe & Herb & $\begin{array}{l}\text { Rhizome, } \\
\text { tuber }\end{array}$ & $\begin{array}{l}\text { Fever; heart pain; stomach ulcers; skin diseases; } \\
\text { leucoderma }\end{array}$ & General & Ram et al. 2004 \\
\hline Zingiber spectabile Griff. & Herb & Rhizome & $\begin{array}{l}\text { Headache; joint pain; bone fracture; abdomen pain; } \\
\text { neurasthenia; indigestion; body ache; jaundice }\end{array}$ & General & Chander et al. 2015 \\
\hline Zingiber zerumbet (L.) Roscoe ex Sm. & Herb & Rhizome & Stomach problems; dysuria; headache; fever; indigestion & General & Chander et al. 2015 \\
\hline
\end{tabular}




\begin{tabular}{|l|l|l|l|l|}
\hline Balanites aegyptiaca (L.) Delile & $\begin{array}{l}\text { Shrub to } \\
\text { tree }\end{array}$ & $\begin{array}{l}\text { Leaves, } \\
\text { fruit, seed, } \\
\text { root }\end{array}$ & Malaria; asthma; healthy foetus; edible & $\begin{array}{l}\text { Foetus } \\
\text { (pregnant } \\
\text { women); } \\
\text { General }\end{array}$ \\
\hline Balanites roxburghii Planch. & Tree & $\begin{array}{l}\text { Flower, fruit, } \\
\text { bark, stem }\end{array}$ & Pertussis; Corneal opacity; Ephemeral fever & General \\
\hline Fagonia bruguieri DC. & Shrub & Whole plant & Snakebite & Chopda and Mahajan 2009 \\
\hline Fagonia indica Burm. f. & Shrub & Whole plant & Fever; cold; cough & General \\
\hline Tribulus terrestris L. & Herb & Whole plant & $\begin{array}{l}\text { Aphrodisiac; impotency; irritations; cough; diuretic; } \\
\text { respriratory problems; fever; headache; heel cracks; } \\
\text { insecticidal; wormicidal; oral health care; kidney stones; } \\
\text { leucorrhea; urinary disorders; wound; gleets; } \\
\text { spermodiarrhoea; veneral diseases }\end{array}$ & $\begin{array}{l}\text { General; } \\
\text { men }\end{array}$ \\
\hline
\end{tabular}

Published 11 February 2019

http://dx.doi.org/10.32859/era.18.4.1-112 


\section{Results}

Indian ethnic communities and distribution map The results showed the following number of indigenous communities for the regions investigated: Andhra Pradesh 34, Karnataka 50, Kerala 37 and Tamil Nadu 36. The diversity of ethnic communities mostly found in southern part of India (Figure 1). Other state wise ethnic communities' names are given as supplementary data. Our analysis shows that few ethnic communities, namely Adiyan, Irular, Kuruman and Vitolia etc., are present across various regions.

\section{Medicinal plants used by ethnic communities}

We recorded a total of 2000 medicinal plant species belonging to 1033 genera and 215 families from South Indian ethnic communities. The most widely used source of medicinal plants used for livestock diseases were herbs $34.12 \%$ ( $n=720$ species), followed by trees $26.49 \%$ ( $n=559$ species), shrubs $22.32 \%$ ( $n=471$ species), climbers $8.67 \%$ ( $n=183$ species), grass $2.70 \%$ ( $n=57$ species), orchid $1.75 \%$ ( $n=37$ species) and "other," which includes ferns, epiphytes, bryophytes, algae and creepers $3.93 \%$ ( $n=83$ species) (Figure 2). Percentages are based on the total number of data points i.e., $\mathrm{N}=$ 2110 (because some plants fall under more than a single habit). The most frequently used parts of the plant were leaves (763 spp.), whole plant (494 spp.), root (362 spp.), fruit (297 spp.), bark (248 spp.), seed (163 spp.), stem (122 spp.), flower (116 spp.), tuber (71 spp.) and "other," which includes vegetative buds, husks, nuts, resins, legumes and twigs. Nine families contributed the highest number of medicinal plants (ranging from 57 to 192 species). These were the Fabaceae (192 species), Asteraceae (104 species), Lamiaceae (89 species), Malvaceae (87 species), Apocynaceae (73 species), Acanthaceae (65 species), Rubiaceae (64 species), Poaceae (57 species) and Euphorbiaceae (56 species). Additionally, 16 families had in the range of 20 to 39 medicinal plant species and 190 families had one to 19 medicinal species. The highest number of genera $7.64 \%(n=79)$ were present in the Fabaceae family and the Ficus genus contained $1.4 \%(n=28)$ species (Table 2).

\section{Knowledge documented}

The study documented the different types of disease such as treating malarial fever, cough, cold and chest pain, wound healing, skin-related diseases, respiratory tract infections and typhoid fever, dyspepsia, dysentery, diabetes, itches, piles and jaundice, snake and scorpion bite, skin disease, liver and renal disease, asthma, cancer, cholera and other diseases observed in pregnant women (Table 1). This shows that traditionally most of the ethnic peoples' treatments focused on rheumatism, abortion, asthma, diabetes, skin disease, liver disease, cough, and (that) also many women underwent abortion (Figure 3). The data showed that the medicines were used to target the entire population: men, women and children. The data demonstrates that from ancient times health care included the diagnosis and treatment of diseases on the basis of age as a major factor which is also true with respect to the other livestock and that most plants used were herbs (720 species). The formulation and route of administration differed, depending on the circumstances. In most cases, along with plant crude extracts (bark, leaves, stem), survey respondents used predominantly hot water, honey, jaggery (traditional cane sugar), milk and in a few cases, other medicinal plants, for example, Getonia floribunda Roxb. is taken with roots of Eclipta prostrata (L.) L. to formulate a medicinal combination (Table 1).

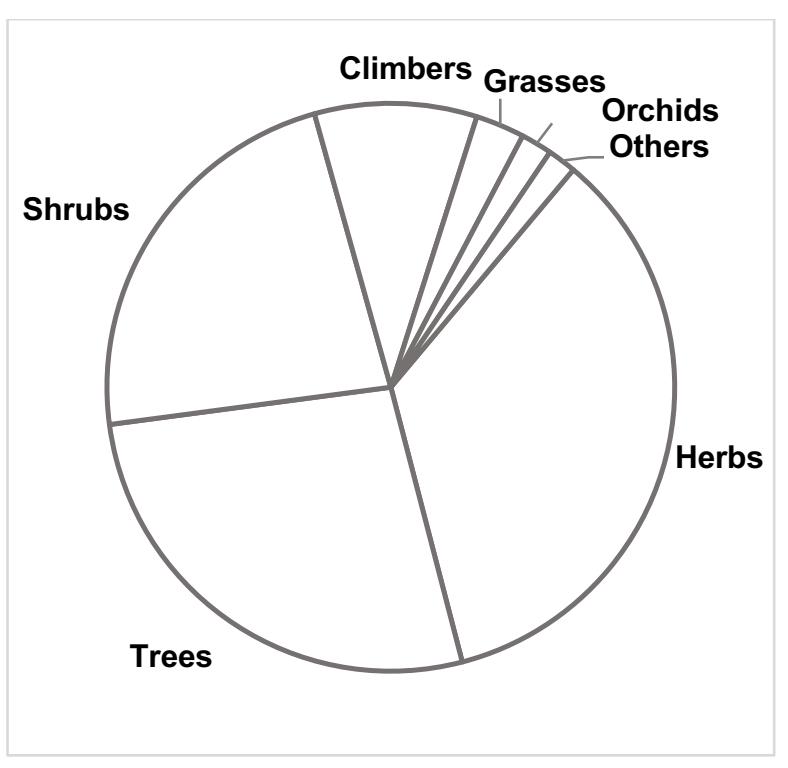

Figure 2. Life forms of plants used for medicinal preparations.

\begin{abstract}
Medicinal plant knowledge between different ethnic communities

The study found region specificity in the usage of medicinal plants. The Malayan ethnic communities in Kerala used Adhatoda beddomei C.B. Clarke species for curing rheumatism; however, the Bhil ethnic community used Alstonia scholaris (L.) $\mathrm{R}$. $\mathrm{Br}$ species for curing the same disease in Karnataka. Further investigated the geographic distribution of medicinal plants available in south India. This allowed us to use the local flora as a guide to the types of medicines available to and subsequently used by each group. Even though some trends are seen, we are not able to definitively conclude that geographic distribution or region-specific availability of medicinal plants used for various diseases is responsible for varied medicinal knowledge between ethnic communities. About 286 different medicinal plants used to cure skin diseases across the ethnic communities and major diseases with respect to species usage were diabetes and rheumatism.
\end{abstract}


Table 2: Taxonomic diversity of medicinal plants used by ethnic communities in south India (19802016).

\begin{tabular}{|l|l|l|l|l|}
\hline \multirow{2}{*}{ Family name } & \multicolumn{2}{l}{ Genera } & \multicolumn{2}{l|}{ Species } \\
\cline { 2 - 5 } & $\mathbf{N}$ & \% & $\mathbf{N}$ & $\%$ \\
\hline Fabaceae & 79 & 7.64 & 192 & 9.60 \\
\hline Asteraceae & 67 & 6.48 & 104 & 5.20 \\
\hline Lamiaceae & 40 & 3.87 & 89 & 4.45 \\
\hline Malvaceae & 35 & 3.38 & 87 & 4.35 \\
\hline Apocynaceae & 43 & 4.16 & 73 & 3.65 \\
\hline Acanthaceae & 31 & 3.00 & 65 & 3.25 \\
\hline Rubiaceae & 36 & 3.48 & 64 & 3.20 \\
\hline Poaceae & 39 & 3.77 & 57 & 2.85 \\
\hline Euphorbiaceae & 23 & 2.22 & 56 & 2.80 \\
\hline Cucurbitaceae & 20 & 1.93 & 39 & 1.95 \\
\hline Convolvulaceae & 11 & 1.06 & 39 & 1.95 \\
\hline Orchidaceae & 23 & 2.22 & 37 & 1.85 \\
\hline Moraceae & 9 & 0.87 & 37 & 1.85 \\
\hline Solanaceae & 12 & 1.16 & 36 & 1.80 \\
\hline Amaranthaceae & 17 & 1.64 & 32 & 1.60 \\
\hline Rutaceae & 16 & 1.54 & 28 & 1.40 \\
\hline Zingiberaceae & 7 & 0.67 & 27 & 1.35 \\
\hline Phyllanthaceae & 9 & 0.87 & 26 & 1.30 \\
\hline Vitaceae & 6 & 0.58 & 23 & 1.15 \\
\hline Anacardiaceae & 13 & 1.25 & 21 & 1.05 \\
\hline Araceae & 14 & 1.35 & 21 & 1.05 \\
\hline Apiaceae & 16 & 1.54 & 20 & 1.00 \\
\hline Menispermaceae & 14 & 1.35 & 20 & 1.00 \\
\hline Combretaceae & 5 & 0.48 & 20 & 1.00 \\
\hline Asparagaceae & 10 & 0.96 & 20 & 1.00 \\
\hline Others & 438 & 42.4 & 767 & 38.35 \\
\hline & & & & \\
\hline & 17 & & \\
\hline
\end{tabular}

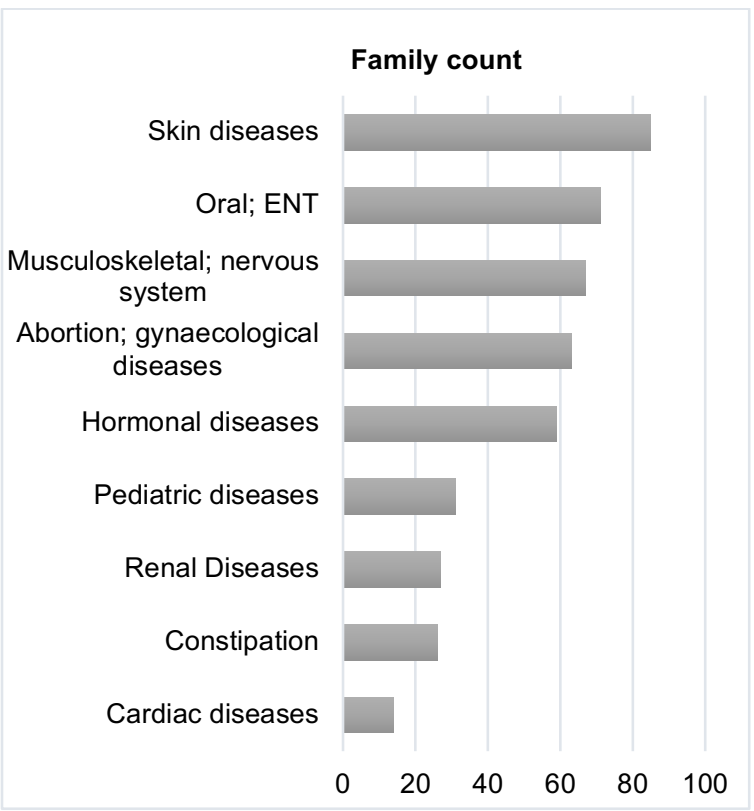

Figure 3. Plant family count based on type of ailment.

\section{Funding agency support}

The study collected 972 peer reviewed articles, of this $30.6 \%(n=298)$ articles funded by various Indian funding agencies. Among these funding agencies, major contributions from the University of Grant Commission (UGC), 32.5\% ( $n=97)$, Indian Council of Medical Research (ICMR), 8.38\% ( $n=25)$, and minor contributions from Science and Engineering Research Board (SERB), 1.67\% $(n=5)$, AYUSH (Department of Ayurveda, Yoga and Naturopathy, Unani, Siddha and Homoeopathy), $1.34 \%(n=4)$ and others $31.8 \%(n=95)$ (Figure 4$)$. These results show that Government of India funding agencies played a major role in ethnobotany studies in India, although even with the support of these funding agencies, some of the ethnic communities' are not yet explored such as Chodhara, Kanivan, Kanyan, Karimpalam, Kudiyam Melekudi, Malai Vedan, Palliayar, Mala Vettuvan, Malapanickar, and Vettakuruman. Having said that, it is promising that ethnic community research increased from 1995 to 2016 (Figure 5).

\section{Informant consensus analysis}

Our study archived the presence of 2000 medicinal plants in southern states of India, many of which are extensively distributed throughout India. Their medicinal values were recorded from various indigenous communities. Classification based on the treatment for a type of health resulted in 9 different categories and $F_{I C}$ value for each was calculated (Table 3). The analysis showed that highest levels of consensus were for the musculoskeletal and neurological category with $F_{I C}=0.553$ and general health with a value of 0.511, followed by dermatological, endocrinological, nephrological, gynaecological, odontological and ENT categories. The least agreement was recorded in cardiovascular illness with $\mathrm{F}_{\mathrm{IC}}=0.083$, depicting the diverse array of usage of ecological resources among the communities and suggests their applications in pharmaceutical industry.

\section{Discussion}

Indigenous communities always had a vital interest in plants, which served as shelter, food and clothing. Plants contain numerous and unknown pharmacologically active compounds and each plant has its own unique combination and properties. Indigenous people rely upon the restorative power of plants primary healthcare and also as their source of income (for currency and exchange). Local ethnic communities in south India have, over time, developed a profound knowledge for utilizing plants, and produced their own medicine and medical knowledge in various regions, based on their unique culture. Many of the ethnic communities live in mountainous or forest areas with low income levels and limited allopathic healthcare. As a result they developed a long history for practicing medicine with natural materials. Some commonly known medicinal traditions come from the Adiyan, Irular, Kattunayakan, Kurumans, Malasar, Palliyan, Paniyan etc. 


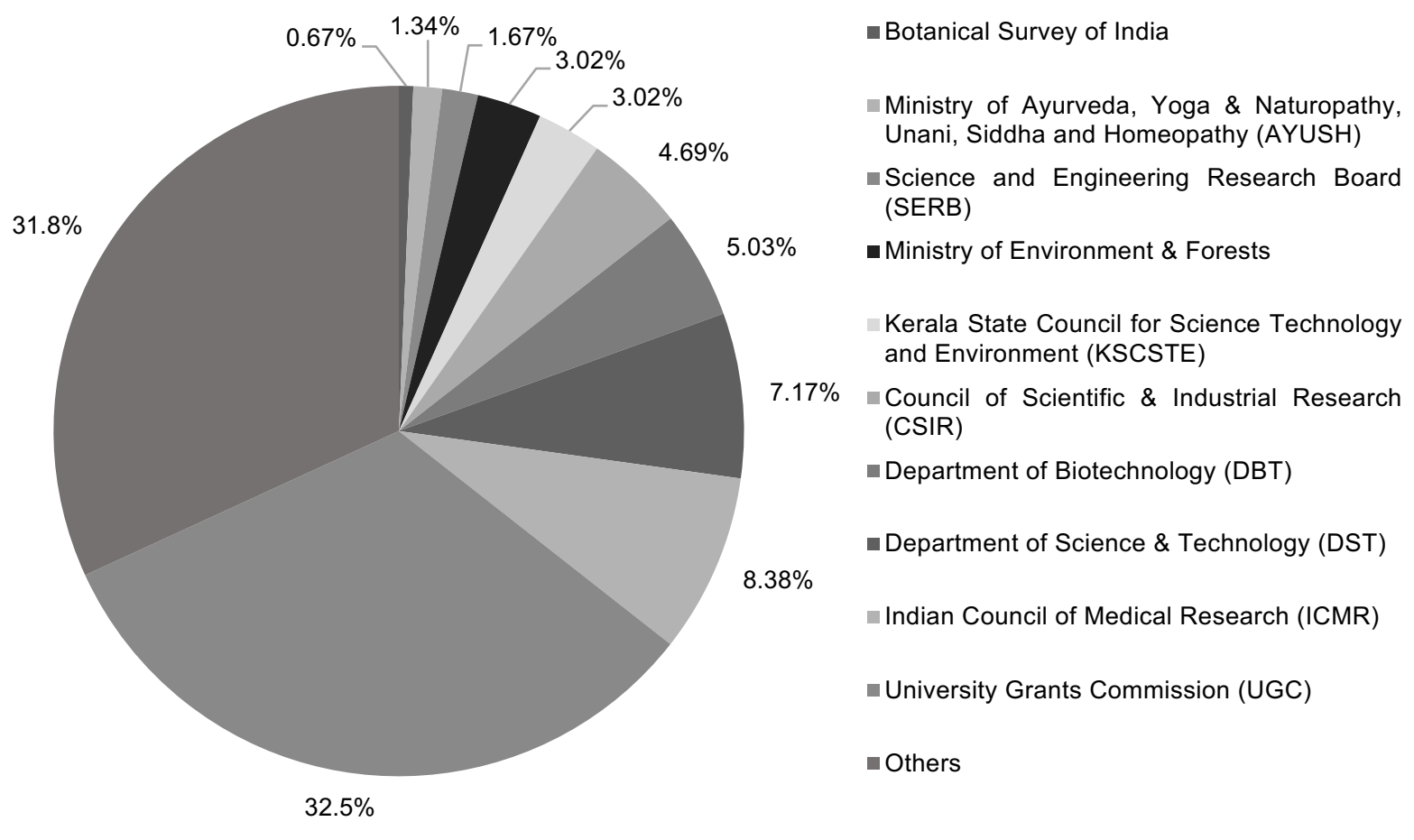

Figure 4. Funding for research on ethnobotany and ethnic affairs.

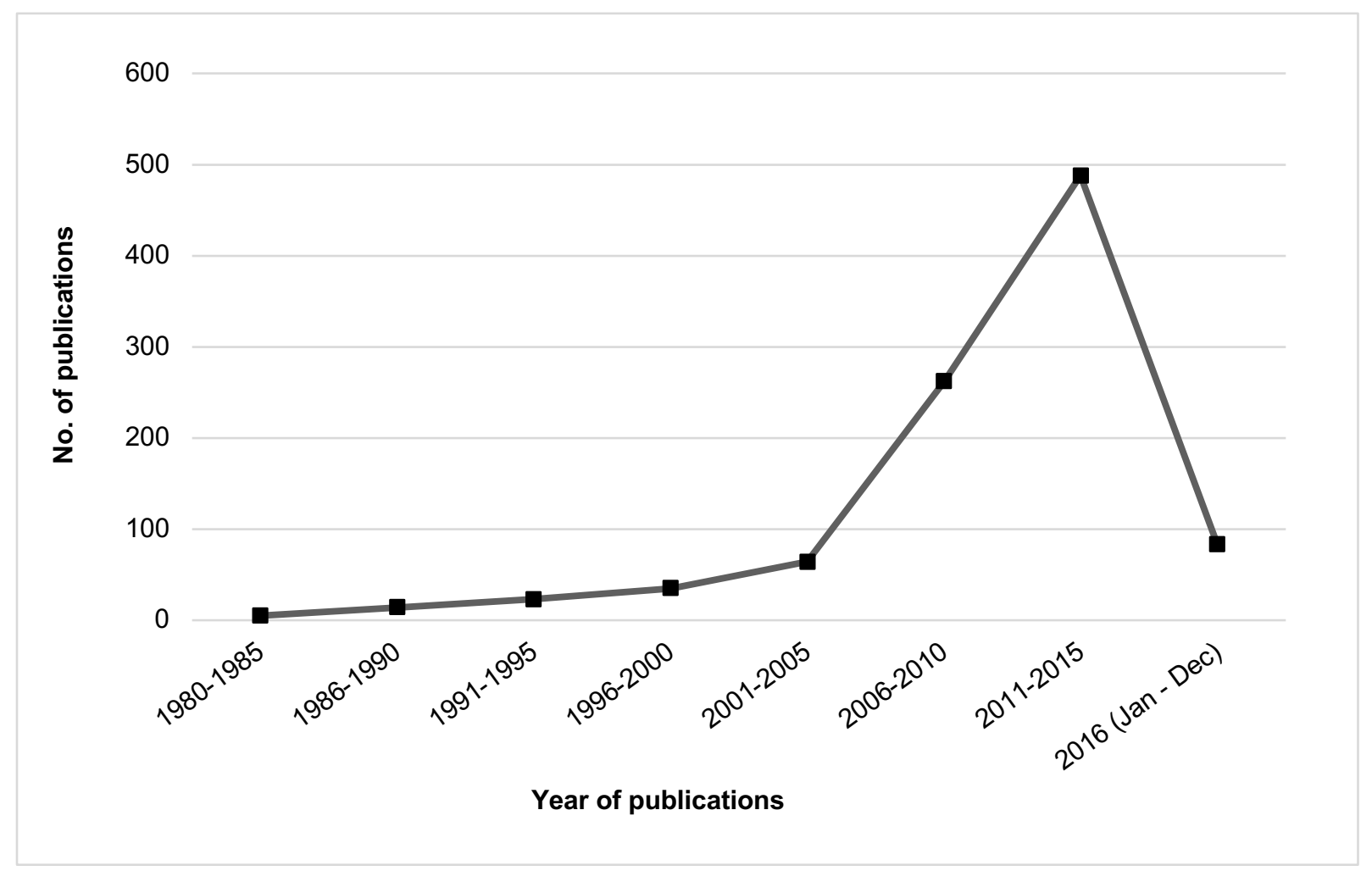

Figure 5. The number of publications reported ethnic communities' studies in India 
Table 3. Informant consensus factor analysis ( $\left.F_{I C}\right)$

\begin{tabular}{|l|l|l|l|}
\hline Category of use & $\begin{array}{l}\text { Use } \\
\text { reports } \\
\mathbf{N}_{\text {ur }}\end{array}$ & $\begin{array}{l}\text { No. of } \\
\text { Taxa } \\
\mathbf{N}_{\mathbf{t}}\end{array}$ & $\begin{array}{l}\text { Informant } \\
\text { consensus } \\
\text { factor } \\
\mathbf{F}_{\mathrm{IC}}\end{array}$ \\
\hline $\begin{array}{l}\text { Musculoskeletal and } \\
\text { neurological }\end{array}$ & 326 & 146 & 0.553 \\
\hline General health & 1241 & 607 & 0.511 \\
\hline Dermatological & 538 & 286 & 0.469 \\
\hline Endocrinological & 226 & 138 & 0.391 \\
\hline Nephrological & 62 & 40 & 0.360 \\
\hline Gynaecological & 166 & 110 & 0.339 \\
\hline $\begin{array}{l}\text { Odontological and } \\
\text { ENT }\end{array}$ & 166 & 116 & 0.303 \\
\hline Gastrointestinal & 67 & 48 & 0.287 \\
\hline Cardiovascular & 37 & 24 & 0.083 \\
\hline
\end{tabular}

Most of the species listed are used in two or more disease groups, such as snake bite, scorpion bite, treating malaria, cough and cold, fever, and aches and pains. Major diseases treated included skin diseases, rheumatism and diabetes, which pose the most serious challenge to primary health care in south India. Most species used to treat these were recently reported in Indian traditional medicine (Ismail \& Leelavathi 2011, Manjula et al. 2013, Patel et al. 2014). A few pharmacological studies regarding species used in indigenous medicine have also been carried out (Pandey \& Negi 2016). Drawn from traditional knowledge, these extensively used species have been shown to contain active compounds and extracts showing therapeutic effects. A list of these studies is shown below:

- Antimicrobial activity and antifungal activity for Senna multiglandulosa (Jacq.) H.S. Irwin \& Barneby (Kanthasamy Kalaichelvi 2016)

- Antiseptic activity for Adenia hondala (Gaertn.) W.J. de Wilde, Atalantia monophylla DC. Barleria acuminata Wight ex Nees, Barleria prionitis L. Arisaema leschenaultii Blume, Bidens biternata (Lour.) Merr. \& Sherff (Arjunan et al. 2016, Bhat et al. 2012, Das et al. 2013, Jain et al. 2009, Kumar et al. 2007, Lingaraju et al. 2013, Pradheeps \& Poyyamoli 2013)

- Antidiabetic activity for Annona squamosa L. Catharanthus roseus (L.) G. Don, Areca catechu L. Cajanus cajan (L.) Millsp. (Dansi et al. 2008, Kumar \& Pullaiah 1999, Lingaraju et al. 2013, Mahishi et al. 2005)

- Anti-inflammatory activity for Cordia africana Lam., Commelina communis L. Anisomeles malabarica (L.) R.Br. ex Sims, Andrographis alata (Vahl) Nees (Chifundera 2001, Kshirsagar \& Singh 2001, Suganthi \& Libina 2015, Valsaraj et al. 1997)
- $\quad$ Antipyretic activity for Andrographis alata (Vahl) Nees, Justicia adhatoda L. Cereus pterogonus Lem, Cinnamomum verum J. Presl, Corchorus aestuans L. (Bhat et al. 2014, Dansi et al. 2008, Kanthasamy Kalaichelvi 2016, Kshirsagar \& Singh 2001)

- Antitumor activity for Calophyllum inophyllum L., Cassia fistula L. Bauhinia purpurea L. Bombax ceiba L. (Bhat et al. 2014, Ignacimuthu et al. 2009, Namsa et al. 2009, Sharma et al. 2013)

In a few types of ailments the usage of specific plants varied between ethnic communities. For example, epilepsy treated by Adiyans using Ipomoea eriocarpa R. Br., whereas Eravallan and Bhil communities used Calotropis procera (Aiton) Dryand. and Vitex negundo L. respectively. Similarly, Adiyan community used Mucuna pruriens (L.) DC., Santalum album L. for blood purification but Irular used Cynodon dactylon (L.) Pers., Senna auriculata (L.) Roxb., Boerhaavia elongata Brandegee; Goud used Woodfordia fruticosa (L.) Kurz and Hakkipikki communities used Ipomoea aquatica Forssk. for the same. Cholera was cured by the Jenu Kuruba community using Alstonia scholaris (L.) R. Br. but Anacardium occidentale L. was used by the Koraga community. Some plants like Jasminum syringifolia Wall.exG.Don and Tabernaemontana crispa Roxb. used as sole medicines to cure malaria, cough and fever.

In this study, we found that ethnic group research grants were mainly given by the University Grant Commission (UGC), India (32.5\%). In India, main research funding agencies or government bodies are Department of Biotechnology (DBT), Department of Science and Technology (DST), Council of Scientific and Industrial Research (CSIR), University Grant Commission (UGC) and Indian Council of Medical Research (ICMR). Apart from these, Botanical Survey of India (BSI), Ministry of Environment and Forests, AYUSH (Department of Ayurveda, Yoga and Naturopathy, Unani, Siddha and Homoeopathy), Indian Council of Agricultural Research (ICAR) and others. Others include that Indian National Science Academy, Kerala State Council for Science Technology and Environment (KSCSTE) and State universities and forestry departments (Figure 4). Even with these funding agencies support, some of the ethnic group's research were not exposed, such as Chodhara, Kanivan, Kanyan, Karimpalam, Kudiyam Melekudi, Malai Vedan, Palliayar, Mala Vettuvan, Malapanickar, and Vettakuruman. However ethnic group research increased from 1995 to 2016 (Figure 5).

A total of 105 ethnic groups were identified within the southern India population along with an extensive variety of names for therapeutic plant species used by these groups. This showed the centrality they are granted by various groups regarding their flexibility and far reaching dispersion. Even though there is a variation in the vernacular names, this promising knowledge of diverse communities is focussed towards their central ethnomedicinal practices. 
Separate communities such as Adiyans, Kurumans, Paniyans and Kurichans used a common plant Alpinia calcarata (Haw.) Roscoe for treating fungal infections which depicts they might have descended from the common place or an exchange of knowledge between their ancestors. The sociolinguistic scope of the vernacular classification implies a typical chronicled and social legacy particular to the nation, and in addition a mutual store of natural learning which rises above environmental, social, and political limits. Also, the presence of a shared view of wellbeing and sickness among ethnically different populations is significant for the exchange, observation and protection of restorative species (Otieno et al. 2015). The present country dataset of India medicinal plants demonstrates the great diversity of botanical knowledge held by local communities and ethnic people, and a significant overlap in terms of health-related applications across the ethnic group.

The present investigation highlights the distribution of research on India's medicinal flora by examining various disregarded and underrepresented issues. Firstly, the species documented for the purpose of this study must be conserved and further ethnobotanical research needs to be carried out. Secondly, research on ethnic groups and medicinal flora has to be done more robustly so as to gain even the smallest, but valuable, ethnic knowledge of India. Finally, emphasis must be placed on research towards multi-disciplinary investigation of local perceptions of disease and the population's healthseeking behaviour for a better understanding of the capability of ethnobotanical and phytotherapeutic knowledge as a public health resource. This explorative survey underscores the need to document indigenous healing methods and practices for common health conditions and urges additional scientific research on the species recorded to determine their efficacy and their safety. The need for regulation of the sector and the benefits of value chains could also assist in promoting bridges and fostering relationships between indigenous medicine and the country's primary health care services, as recommended by the WHO (2013).

\section{Conclusions}

The southern part of India observed to be rich in plant species used for a wide range of purposes. Our research showed a clear need for documentation of plants used to develop herbal medicines in indigenous communities. This study will not only serve as a source of information but will also help to make the knowledge accessible for further drug screening and development, and, at the same time, underlines the need for biodiversity conservation of this traditional wealth. The regional overlap of many indigenous species, the consensual nature of disease groups based upon local perceptions of health conditions, and the relevance of local vernacular names which enhance the potential for the circulation and transmission of ethnobotanical and therapeutic knowledge. The ethnic people's understanding and the use of medicinal plants are found to be grounded on traditional background. Knowledge transfer practice among the ethnic communities is reported to be received from generation to generation. The people of ethnic communities often commented that most of the medicinal plants were under threat due to increasing anthropogenic influence on the natural habitat of the plants. Even though these indigenous communities contribute to a very small proportion of the country's population, their knowledge is of a great cultural, social, and potentially economic value. This must be protected and utilized in a conscientious manner and at the same time we have to take steps to make sure there is no disturbance to its ties to the communities.

\section{Declarations}

List of abbreviations: Not applicable. Ethics approval and consent to participate: Not applicable.

Consent for publication: Not applicable.

Availability of data and materials: Please contact the author for data requests.

Competing interests: The authors declare that they have no competing interests.

Conflict of interest: The authors declare that they have no conflict of interests.

Author Contributions: Conceptualization and designing the study; Santhosh Kumar, Data collection and formal analysis; Santhosh Kumar, Krishna Chaitanya, Validation and Visualization; Santhosh Kumar, Andrew Semotiuk and Krishna Chaitanya, Manuscript writing: Santhosh Kumar, Review \& editing: Krishna, Andrew Semotiuk, and Santhosh Kumar.

Acknowledgements: Authors would like to acknowledge Ms. Kesang Choden Bhutia, CES, IISc, India and also Mr. Bipin Charles, ATREE, India for help in generating the thematic Indian map for this study. We thank UC MEXUS for support of one author.

\section{Author Affiliations:}

Santhosh Kumar $\mathrm{JU}^{1,2 *}$, Krishna Chaitanya $\mathrm{MJ}^{3}$, Andrew J Semotiuk ${ }^{4}$ and Krishna V ${ }^{1}$

${ }^{1}$ Department of Post Graduate Studies and Research in Biotechnology, Jnanasahyadri, Kuvempu University, Shankaraghatta, Shimoga 577451, India

${ }^{2}$ School of Ecology and Conservation, University of Agricultural Sciences, GKVK, Bangalore-560065, India

${ }^{3}$ St. Joseph's College (Autonomous), Bangalore 560027, India

${ }^{4}$ Department of Botany and Plant Sciences, University of California, Riverside, California, 92521, USA 


\section{Literature cited}

Abraham J, Thomas TD. 2012. Antibacterial activity of medicinal plant Cyclea peltata (Lam) Hooks \& Thoms. Asian Pacific Journal of Tropical Disease 2:S280-S284.

Acharya KP, Kaphle K. 2015. Ethnomedicinal plants used by yak herders for management of health disorders. Journal of Intercultural Ethnopharmacology 4(4):270-276.

Adhikari BS, Babu MM, Saklani PL, Rawat GS. 2010. Medicinal plants diversity and their conservation status in Wildlife Institute of India (WII) campus, Dehradun. Ethnobotanical Leaflets 14:46-83.

Ajesh TP, Kumuthakalavalli R. 2012. International Journal of Pharmacy \& Life Sciences 3(12):22132219.

Ajesh TP, Naseef SA, Kumuthakalavalli R. 2012. Ethnobotanical documentation of wild edible fruits used by Muthuvan tribes of Idukki, Kerala-India. International Journal of Pharma and Bio Sciences 3(3):479-487.

Alagesaboopathi C. 2012. Ethnomedicinal uses of Andrographis elongata T. And.-An endemic medicinal plant of India. International Journal of Recent Scientific Research 3(4):231-233.

Alam SS, Munira S, Habib MA, Sultana SS. 2012. Karyotype and RAPD Analysis in Common and Rare Forms of Lasia spinosa (L.) Thwaites. Cytologia 77(4):499-505

Al-Asmari A, Manthiri RA, Abdo N, Al-Duaiji FA, Khan HA. 2016. Saudi medicinal plants for the treatment of scorpion sting envenomation. Saudi Journal of Biological Science 24(6):1204-1211.

Anusuya N, Raju K, Manian, S. 2010. Hepatoprotective and toxicological assessment of an ethnomedicinal plant Euphorbia fusiformis Buch.Ham. ex D. Don. Journal of Ethnopharmacology 127(2):463-467.

Arjunan V, Thekkan S, Ali MA, Senniayappan TS, Subramaniyam P. 2016. Ethnomedicinal assessment of Irula tribes of Walayar valley of Southern Western Ghats, India. Saudi Journal of Biological Sciences 25(4):760-775.

Asha V, Pushpagadan P. 1998. Ethnomedical and pharmacognostic investigations on Rhaphidophora pertusa Schott. Ancient Science of Life 17(3):235238.

Awate PD, Gaikwad DK. 2014. Influence of growth regulators on secondary metabolites of medicinally important oil yielding plant Simarouba glauca DC. under water stress conditions. Journal of Stress Physiology \& Biochemistry 10(1):222-229.

Ayyanar M, Ignacimuthu S. 2005. Traditional knowledge of Kani tribals in Kouthalai of Tirunelveli hills, Tamil Nadu, India. Journal of Ethnopharmacology 102(2):246-255.
Ayyanar M. 2013. Traditional Herbal Medicines for Primary Healthcare among Indigenous People in Tamil Nadu, India. Journal of Homeopathy \& Ayurvedic Medicine 2(5):1-7.

Ayyanar M, Sankarasivaraman K, Ignacimuthu S. 2008. Traditional healing potential of Paliyars in southern India. Ethnobotanical Leaflets 12:311-317.

Balick MJ. 2007. Traditional knowledge: Lessons from the past, lessons for the future. In Biodiversity and the Law: Intellectual Property, Biotechnology and Traditional Knowledge. Edited by CR McManis. Earthscan, London, U.K., 280-296.

Banumathi B, Vaseeharan B. 2015. A Report on Medicinal Plants Used in Ethno Veterinary Practices of Toda Tribe in the Nilgiri Hills. Journal of Veterinary Science \& Technology 6(5):1-6.

Basha SKM, Umamaheswari P, Rambabu M, Savitramma N. 2011. Ethnobotanical Study of Mamandur Forest (Kadapa-Nallamali Range) in Eastern Ghats, Andhra Pradesh, India. Journal of Phytology 3(10):44-47.

Bhagat RB, Chambhare M, Mate S, Dudhale A, Zaware BN. 2016. Prospective wild edible fruit plants from part of northern Western Ghats (NWG), Mulshi (MS), India. Journal of Medicinal Plants 4(1):15-19.

Bhakshu LM, Ratnam KV, Raju RR. 2016. Anticandidal activity and phytochemical analysis of certain medicinal plants from Eastern Ghats, India. Indian Journal of Natural Products and Resources 7(1):25-31.

Bhandary MJ, Chandrashekar KR, Kaveriappa KM. 1995. Medical ethnobotany of the Siddis of Uttara Kannada district, Karnataka, India. Journal of Ethnopharmacology 47(3):149-158.

Bharathi LK, John KJ. 2013. Ethnobotany and Nutritive Value. In Momordica genus in Asia-An Overview. Springer, India, 61-76.

Bhardwaj M, Bharadwaj L, Trigunayat K, Trigunayat MM. 2011. Insecticidal and wormicidal plants from Aravalli hill range of India. Journal of Ethnopharmacology 136(1):103-110.

Bharti M, Pravesh R. 2012. Antibacterial activity of Lygodium flexuosum (L.) Sw. and Ampelopteris prolifera (Retz.) Copel of Ranchi, District of Jharkhand Ecoscan 1:397-401.

Bhaskar A, Samant LR. 2012. Traditional medication of Pachamalai Hills, Tamilnadu, India. Global Journal of Pharmacology 6(1):47-51.

Bhat P, Hedge GR, Hedge G, Mulgund GS. 2013. Ethnomedicinal plants to cure skin diseases an account of the traditional knowledge in the coastal parts of central Ghats, Karnataka, India. Journal of Ethnopharmacology 151:493-502.

Bhat P, Hegde G, Hegde GR. 2012. Ethnomedicinal practices in different communities of Uttara Kannada district of Karnataka for treatment of wounds. Journal of Ethnopharmacology 143(2):501-514. 
Bhat P, Hegde GR, Hegde G, Mulgund GS. 2014. Ethnomedicinal plants to cure skin diseases, An account of the traditional knowledge in the coastal parts of Central Western Ghats, Karnataka, India. Journal of Ethnopharmacology 151(1):493-502.

Bhattacharjee A, Shashidhara SC, Saha S. 2013. Phytochemical and ethno-pharmacological profile of Desmodium gangeticum (L.) DC.: A review. International Journal of Biomedical Research 4(10):507-515.

Bhogaonkar PY, Kadam VN. 2006. Ethnopharmacology of Banjara tribe of Umarkhed taluka, district Yavatmal, Maharashtra for reproductive disorders. Indian Journal of Traditional Knowledge 5:336-41.

Bhuvaneswari R, Ramanathan R, Krishnapriya $\mathrm{P}$, Madheswaran A, Dhandapani R. 2015. Survey of wild tuberous medicinal plants of Kolli hills in Namakkal district, Tamil Nadu, India. International Journal of Herbal Medicine 3(4 Part A):41-48.

Binu S. 2010. Wild edible plants used by the tribals in Pathanamthitta district, Kerala. Indian Journal of Traditional Knowledge 9(2):309-312.

Binu S. 2011. Medicinal Plants used for treating body pain by the tribals in Pathanamthitta district, Kerla, India. Indian Journal of Traditional knowledge 10(3):547-549.

Bosco FG, Arumugam R. 2012. Ethnobotany of irular tribes in redhills, tamilnadu, India. Asian Pacific Journal of Tropical Disease 2:S874-S877.

Case RJ, Pauli GE, Soejarto DD. 2005. Factors in maintaining indigenous knowledge among ethnic communities of Manus Island. Economic Botany 59:356-365

Chakraborty R, De B, Devanna N, Sen S. 2012. North-East India an ethnic storehouse of unexplored medicinal plants. Journal of Natural Product and Plant Resources 2(1):143-152.

Chandra S, Chandra D, Barh A, Pandey RK, Sharma IP. 2016. Bryophytes: Hoard of remedies, an ethnomedicinal review. Journal of Traditional and Complementary Medicine 7(1):94-98.

Chenniappan K, Kadarkarai M. 2010. In vitro antimalarial activity of traditionally used Western Ghats plants from India and their interactions with chloroquine against chloroquine-resistant Plasmodium falciparum. Parasitology Research 107(6):1351-1364.

Chifundera K. 2001. Contribution to the inventory of medicinal plants from the Bushi area, South Kivu Province, Democratic Republic of Congo. Fitoterapia 72(4):351-368.

Chithra M, Prabhu Kumar KM, Geetha SP. 2016. A comparative study on ethanobotanical usage of plants for twenty selected diseases by six tribal communities in Malappuram district. Journal of Herbal Medicine 4(4):108-113.
Chopda MZ, Mahajan RT. 2009. Wound healing plants of Jalgaon district of Maharashtra state, India. Ethnobotanical Leaflets 13:1-32.

Dansi A, Adjatin A, Adoukonou-Sagbadja H, Faladé V, Yedomonhan H, Odou D, Dossou B. 2008. Traditional leafy vegetables and their use in the Benin Republic. Genetic Resources and Crop Evolution 55(8):1239-1256.

Das AK, Stalin N, Muthuperumal C, Swamy PS. 2013. Wild plants used by Muthuvan and Kattunaikkan tribal communities of Palakkayam settlement in Nilambur of Malappuram district, Kerala. Medicinal Plants-International Journals of Phytomedicines and Related Industries 5(2):82-89.

Das B, Talukdar AD, Choudhury MD. 2014. A few traditional medicinal plants used as antifertility agents by ethnic people of Tripura, India. International Journal of Pharmacy and Pharmaceutical Sciences 6(3):47-53.

Das PK, Misra MK. 1988. Some ethnomedicinal plants of Koraput district Orissa. Ancient Science of Life 8(1):60-67.

Deepa KC, Jose M, Prabhu V. 2011. Ethnomedicinal Practices For Oral Health And Hygiene Of Tribal Population Of Wayanad, Kerala. International Journal of Research in Ayurveda \& Pharmacy 2(4):1246-1250.

Deepak P, Gopal GV. 2014. Ethnomedicinal Practices Of Kurumba Tribes, Niligiri District, Tamil Nadu, India, In Treating Skin Diseases. Global Journal of Research on Medicinal Plants \& Indigenous Medicine 3(1):8-16.

Devi VM, Kalirajan K, Prasad PN. 2010. Existence of Vitamin C Fruit Sources in the Kani Tribal Settlements of South TamilNadu. Journal of Phytology 2(4):27-32.

Dey A, De JN. 2012. Traditional use of plants against snakebite in Indian subcontinent: a review of the recent literature. African Journal of Traditional, Complementary and Alternative Medicines 9(1):153174.

Dey A, Gupta B, De JN. 2012. Traditional phytotherapy against skin diseases and in wound healing of the tribes of Purulia district, West Bengal, India. Journal of Medicinal Plants Research 6(33):4825-483.

Dhal Y, Sahu RK, Deo B. 2011. Ethno medicinal survey of Koraput District, Odisha: An Update. Journal of Pharmacy Research 4(11):4142-4145.

Dhanabal SP, Lall N, Pavithra N, Chaitanya MVNL. 2015. Natural products as an important leads for discovery of new antitubercular agents: A review. International Journal of Pharmacy and Pharmaceutical Sciences 7(11):2-7.

Dhanabalan R. 2008. In vitro Phytochemical Screening and Antibacterial Activity of Organic Leaf Extracts of Spathodea campanulata P. Beauv 
against Hospital Isolated Bacterial Strains. Ethnobotanical Leaflets 12:1022-1028.

Dhivya SM, Kalaichelvi K. 2015. Studies on Eth Karamadai Range of Western Ghats, Tamil Nadu, India. International Journal of Chemistry and Pharmaceutical Sciences 3(11):2116-2124.

Dileep P, Nair GG. 2015. Taxonomic and ethnobotanical studies of grasses used by tribals of Wayanad District, Kerala, South Western Ghats of India. Journal of Global Biosciences 4(5):2212-2235.

Dubey D, Sahu MC, Rath S, Paty BP, Debata NK, Padhy RN. 2012. Antimicrobial activity of medicinal plants used by aborigines of Kalahandi, Orissa, India against multidrug resistant bacteria. Asian Pacific Journal of Tropical Biomedicine 2(2):S846-S854.

Duraipandiyan V, Ayyanar M, Ignacimuthu S. 2006. Antimicrobial activity of some ethnomedicinal plants used by Paliyar tribe from Tamil Nadu, India. BMC Complementary and Alternative Medicine 6(1):3541.

Dushing YA, Patil DA. 2011. Studies on ethnomedicine in Buldhana district of Maharashtra (India). Journal of Phytology 2(12):35-41.

Edayadulla N, Ramesh P. 2012. Antibacterial activity of various stem extracts of Dalbergia coromandeliana. Asian Pacific Journal of Tropical Biomedicine 2(3):S1388-S1391.

Eneh PN, Horien CL, Ashraf W, Bakken J, Bath JL. 2013. A randomized survey of medicinal plants used as natural remedies by the local people of Manikganj district of Bangladesh to treat intestinal worms. Journal of Medicinal Plants Research 7(9):543-550.

Farnsworth NR, Akerele O, Bingel AS, Soejarto DD, Guo Z. 1985. Medicinal plants in therapy. Bulletin World Health Organisation 63:965-981.

Gailea R, Bratawinata AA, Pitopang R, Kusuma I. 2016. The Use Of Various Plant Types As Medicines By Local Community In The Enclave Of The LoreLindu National Park Of Central Sulawesi, Indonesia. Global Journal of Research on Medicinal Plants \& Indigenous Medicine 5(1):29-40.

Gairola S, Sharma J, Gaur RD, Siddiqi TO, Painuli RM. 2013. Plants used for treatment of dysentery and diarrhoea by the Bhoxa community of district Dehradun, Uttarakhand, India. Journal of Ethnopharmacology 150(3):989-1006.

Gaitonde BB, Kurup PN. 2005. Regional overview: South-East Asia region. In WHO Global Atlas of Traditional, Complementary and Alternative Medicine. Edited by G Bodeker, CK Ong, C Grundy, G Burford, K Shein. WHO Centre for Health and Development, Kobe, Japan, 75-82.

Gavali D, Sharma D. 2004. Traditional knowledge and biodiversity conservation in Gujarat. Indian Journal of Traditional Knowledge 3(1):51-58.

Gayakvad P, Jadeja DB, Thakre B, Bhalawe S, Nayak D. 2014. Ethno-veterinary medicinal plants of mahal village of dang district, Gujarat, India. Research in Environment and Life Sciences 7(2):99100.

Ghanthi Kumar S, Manickam VS. 2008. Ethnobotanical Utilization of Poecilineron pauciflorum Bedd. by the Kani Tribes of Agasthiamalai, Western Ghats, Tamil Nadu, India. Ethnobotanical Leaflets 12:719-722.

Gohain N, Prakash A, Gogoi K, Bhattacharya DR, Sarmah NP, Dahutia C, Kalita MC. 2015. An Ethnobotanical Survey Of Anti-Malarial Plants In Some Highly Malaria Affected Districts Of Assam. International Journal of Pharmacy and Pharmaceutical Sciences 7(9):147-152.

Goswami D, Mukherjee PK, Kar A, Ojha D, Roy S, Chattopadhyay D. 2016. Screening of ethnomedicinal plants of diverse culture for antiviral potentials. Indian Journal of Traditional Knowledge 15(3):474-481.

Harsha VH, Hebbar SS, Shripathi V, Hegde GR. 2003. Ethnomedicobotany of Uttara Kannada District in Karnataka, India-plants in treatment of skin diseases. Journal of Ethnopharmacology 84(1):3740.

Hebbar SS, Harsha VH, Shripathi V, Hegde GR. 2004. Ethnomedicine of Dharwad district in Karnataka, Indiaplants used in oral health care. Journal of Ethnopharmacology 94(2):261-266.

Heinrich M, Ankli A, Frei B, Weimann C, Sticher O. 1998. Medicinal plants in Mexico: Healers'consensus and cultural importance. Social Science \& Medicine 47:1859-1871.

Hills D. 2010. Ethnopharmacological studies on the Medicinal Plants used by Tribal Inhabitants of Kottur. Environment \& We: An International Journal of Science and Technology 5(2010):57-64.

Hiremath VT, Taranath TC. 2010. Traditional phytotherapy for snake bites by tribes of Chitradurga District, Karnataka, India. Ethnobotanical Leaflets 14:120-125.

Horo S, Topno S. 2015. Study and analysis of nutritional value of some wild and semi wild edible plants consumed by "HO" tribes of W. Singhbhum district, Jharkhand, India. International Journal of Herbal Medicine 3(5 Part A):25-32.

Hota NP, Padhi MM. 2003. Typical uses of certain common and uncommon plants. Ancient Science of Life 23(1):1-7.

Ignacimuthu S, Pavunraj M, Duraipandiyan V, Raja $\mathrm{N}$, Muthu C. 2009. Antibacterial activity of a novel quinone from the leaves of Pergularia daemia (Forsk.), a traditional medicinal plant. Asian Journal of Traditional Medicine 4(1):36-40.

Ignacimuthu S, Ayyanar M, Sankarasivaraman K. 2008. Ethnobotanical study of medicinal plants used by Paliyar tribals in Theni district of Tamil Nadu, India. Fitoterapia 79(7):562-568. 
Ishola IO, Akindele AJ, Adeyemi OO. 2011. Analgesic and anti-inflammatory activities of Cnestis ferruginea Vahl ex DC (Connaraceae) methanolic root extract. Journal of Ethnopharmacology 135(1):55-62.

Ismail SM, Leelavathi S. 2011. Evaluation of antioxidant activity of Anisolmeles malabarica $\mathrm{R}$ Brand Clerodendrum serratum L. extracts against rheumatism. Research Journal of Pharmaceutical, Biological and Chemical Sciences 2:488-495.

Israel BA, Schulz AJ, Parker EA, Becker AB. 1998. Review of community-based research: assessing partnership approaches to improve public health. Annual Review Public Health 19(1):173-202.

Iwalewa EO, Iwalewa OJ, Adeboye JO. 2003. Analgesic, antipyretic, anti-inflammatory effects of methanol, chloroform and ether extracts of Vernonia cinerea less leaf. Journal of Ethnopharmacology 86(2):229-234.

Jaganathan GK, Hoa THT, Liu BL. 2016. Ethnobotanical survey of Irular tribes in Pillur valley, Coimbatore, Tamil Nadu (India). International Journal of Herbal Medicine 4(1):1-11.

Jagtap SD, Deokule SS, Pawar PK, Harsulkar AM. 2009. Traditional ethnomedicinal knowledge confined to the Pawra tribe of Satpura hills, Maharashtra, India. Ethnobotanical Leaflets 13:98115.

Jain SC, Jain R, Singh R. 2009. Ethnobotanical survey of Sariska and Siliserh regions from Alwar district of Rajasthan, India. Ethnobotanical Leaflets 13:171-188.

Jain A, Katewa SS, Chaudhary BL, Galav P. 2004. Folk herbal medicines used in birth control and sexual diseases by tribals of southern Rajasthan, India. Journal of Ethnopharmacology 90(1):171-177.

Jain SK. 2004. Credibility of traditional knowledgeThe criterion of multilocational and multiethnic use. Indian Journal of Traditional Knowledge 3(2):137153.

Jayakumar R, Pandalai RC, Nair KKN. 2010. Restoring Natural Capital in the Tropical Dry Deciduous Forests of the Western Ghats of India. Ecological Restoration 28(4):485-492.

Jayanthi P, Aravindhan V, Rajendran A. 2012. Phytotherapeutic Plants Of Madukkarai Hills In The Southern Western Ghats Of Coimbatore District, Tamil Nadu, India. International Journal of Ayurvedic and Herbal Medicine 2(5):807-906.

Jenisha SR, Jeeva S. 2014. Traditional Remedies Used by the Inhabitants of KeezhakrishnanputhoorA Coastal Village of Kanyakumari District, Tamilnadu, India. Medicinal \& Aromatic Plants $3(4): 1-5$.

Joseph JK, Antony VT. 2008. Ethnobotanical investigations in the genus Momordica $L$. in the Southern Western Ghats of India. Genetic Resources and Crop Evolution 55(5):713-721.
Kadhirvel K, Ramya S, Sudha TPS, Ravi AV, Rajasekaran C, Selvi RV, Jayakumararaj R. 2010. Ethnomedicinal survey on plants used by tribals in Chitteri Hills. Environment \& We: An International Journal of Science \& Technology 5:35-46.

Kalaiselvan M, Gopalan RT. 2014. Ethnobotanical Studies on Selected Wild Medicinal Plants used by Irula Tribes of Bolampatty Valley, Nilgiri Biosphere Reserve (NBR), Southern Western Ghats, India. Asian Journal of Pharmaceutical and Clinical Research 7(1):22-26.

Kamble SY, More TN, Patil SR, Pawar SG, Bindurani R, Bodhankar SL. 2008. Plants used by the tribes of Northwest Maharashtra for the treatment of gastrointestinal disorders. Indian Journal of Traditional Knowledge 7(2):321-325.

Kanthasamy-Kalaichelvi SMD. 2016. Medicinal plants used by Irula tribes of Nellithurai Beat, Karamadai Range, Western Ghats, Tamil Nadu, India: An ethnobotanical survey. Journal of Medicinal Plant Research 4(4):270-277.

Kataria S, Kaur D. 2013. Ethnopharmacological approaches to inflammation-exploring medicinal plants. Indian Journal of Natural Products and Resources 4(3):295-305.

Kayani S, Ahmad M, Zafar M, Sultana S, Khan MPZ, Ashraf MA, Hussain J, Yaseen G. 2014. Ethnobotanical uses of medicinal plants for respiratory disorders among the inhabitants of Gallies-Abbottabad, Northern Pakistan. Journal of Ethnopharmacology 156:47-60.

Kennedy SJ. 2006. Commercial Non-timber forest products collected by the tribals in the Palni hills. Indian Journal of Traditional Knowledge 5(2):212216.

Khan AV, Ahmed QU, Mir MR, Shukla I, Khan AA. 2011. Antibacterial efficacy of the seed extracts of Melia azedarach against some hospital isolated human pathogenic bacterial strains. Asian Pacific Journal of Tropical Biomedicine 1(6):452-455.

Khyade MS, Kasote DM, Vaikos NP. 2014. Alstonia scholaris (L.) R. Br. and Alstonia macrophylla Wall. ex G. Don: A comparative review on traditional uses, phytochemistry and pharmacology. Journal of Ethnopharmacology 153(1):1-18.

Kosalge SB, Fursule RA. 2009. Investigation of ethnomedicinal claims of some plants used by tribals of Satpuda Hills in India. Journal of Ethnopharmacology 121(3):456-461.

Krishna MB, Mythili S, Kumar KS, Ravinder B, Murali T, Mahender T. 2011. Ethno botanical survey of medicinal plants in Khammam District, Andhra Pradesh, India. International Journal of Applied Biology and Pharmaceutical Technology 2:366-370.

Krishna NR, Varma YNR, Saidulu C. 2014. Ethnobotanical Studies of Adilabad District, Andhra Pradesh, India. Journal of Pharmacognosy and Phytochemistry 3(1):18-36. 
Krishnaraj MV, Prabu M, Kumuthakalavalli R. 2012. Herbal Abortifacients Used by Mannan Tribes of Kerala, India. International Journal of PharmTech Research 4(3):1015-1017.

Kshirsagar RD, Singh NP. 2001. Some less known ethnomedicinal uses from Mysore and Coorg districts, Karnataka state, India. Journal of Ethnopharmacology 75(2):231-238.

Kshirsagar R, Saklani A. 2007. Ethnomedicinal Plants for Diabetes, Jaundice and Rheumatism in Karnataka and their comparison with Northeast India. Advances in Ethnobotany 95-116.

Kumar PP, Ayyanar M, Ignacimuthu S. 2007. Medicinal plants used by Malasar tribes of Coimbatore district, Tamil Nadu. Indian Journal of Traditional Knowledge 6(4):579-582.

Kumar TD, Pullaiah T. 1999. Ethno-Medico-Botany of Chenchus of Mahaboobnagar District, Andhra Pradesh. Ancient Science of Life 19(1-2):31-35.

Kumar GP, Chaturvedi A. 2010. Ethnobotanical observations of Euphorbiaceae species from Vidarbha region, Maharashtra, India. Ethnobotanical Leaflets 14: 674-680.

Kumar M, Bussmann RW, Mukesh J, Kumar P. 2011. Ethnomedicinal uses of plants close to rural habitation in Garhwal Himalaya, India. Journal of Medicinal Plants Research 5(11):2252-2260.

Kumar P. 2014. Ethno medicinal plants used for oral health care in India. International Journal of Herbal Medicine 2(1):81-87.

Kumar PP, Ayyanar M, Ignacimuthu S. 2007. Medicinal plants used by Malasar tribes of Coimbatore district, Tamil Nadu. Indian Journal of Traditional Knowledge 6(4):579-582.

Kumar TD, Pullaiah T. 1999. Ethno-Medico-Botany of Chenchus of Mahaboobnagar District, Andhra Pradesh. Ancient science of life 19(1-2):31-35.

Latheef A, Kumar SP, Remashree AB. 2014. Ethnomedicine used for treating cuts and wounds by the tribes of Attappady, Kerala. International Journal of Herbal Medicine 2(2):1-8.

Madhu V, Suvartha C. 2009. Ethnobotanical and ethnomedicinal observations in Nirmal Division of Adilabad district, Andhrapradesh, India. Ethnobotanical Leaflets 13:1003-1016.

Madhu V, Swamy TN. 2010. Ethnomedicine against jaundice used by Gond tribes of Adilabad district, Andhra Pradesh, India. Ethnobotanical Leaflets 14:687-693.

Mahishi P, Srinivasa BH, Shivanna MB. 2005. Medicinal plant wealth of local communities in some villages in Shimoga District of Karnataka, India. Journal of Ethnopharmacology 98(3):307-312.

Majumder S, Ashok BK, Nishteswar K. 2013. Evaluation of intestinal transit time of root and leaves of Ipomea sepiaria. Ayu 34(4):430-432.
Mali PY, Bhadane VV. 2011. Ethno-medicinal wisdom of tribals of Aurangabad district (MS), India. Indian Journal of Natural Product Resources 2(1):102-109.

Malla B, Gauchan DP, Chhetri RB. 2015. An ethnobotanical study of medicinal plants used by ethnic people in Parbat district of western Nepal. Journal of Ethnopharmacology 165: 103-117.

Mallikarjuna K. 2015. Ethnomedicine for Rheumatoid arthritis by tribes of Papikondalu forest, Andhra Pradesh, India. American Journal of Ethnomedicine 2(6):373-378.

Maloles JR, Berg K, Ragupathy S, Nirmala BC, Althaf KA, Palanisamy VC, Newmaster SG. 2011. The fine scale ethnotaxa classification of millets in southern India. Journal of Ethnobiology 31(2):262287.

Malviya N, Jain S, Gupta VB, Vyas S. 2011. Indigenous herbal remedies used by tribals of Madhya Pradesh for improving their sexual performance and problem associated with sexuality. International Journal of Research in Ayurveda and Pharmacy 2(2):399-402.

Manikandan PA. 2005. Folk herbal medicine: A survey on the Paniya tribes of Mundakunnu village of the Nilgiri hills, South India. Ancient Science of Life 25(1):21-27.

Manikandan PA, Jayendran M, Rajasekaran CS. 2006. Study of plants used as anti diabetic agents by the nilgiri aborigines. Ancient Science of Life 25(34):101-103.

Manikandan S, Lakshmanan GA. 2014. Ethnobotanical survey of medicinal plants in kalrayan hills, eastern Ghats, Tamil Nadu. International Letters of Natural Sciences 12:111121.

Manithottam J, Francis MS. 2008. Preparation of Maravuri from Antiaris toxicaria (Pers.) Lesch. by Muthuvans of Kerala. Indian Journal of Traditional Knowledge 7(1):74-76.

Manjula RR, Rao JK, Reddi TVVS. 2013. Ethnomedicine for Rheumatism by the Tribals of Khammam District, Andhra Pradesh. Journal of Natural Medicine 13:138-141.

Manorama SS. 2015. Ethnobotanical, Phytochemical and invitro antioxidant activity of medicinal plant Pimenta dioica (I.) Merr.(Myrtaceae) from Attappadi, Palakkad district, Kerala. Research in Pharmacy 4(1):1-7.

Mary HP, Susheela GK, Jayasree S, Nizzy AM, Rajagopal B, Jeeva S. 2012. Phytochemical characterization and antimicrobial activity of Curcuma xanthorrhiza Roxb. Asian Pacific Journal of Tropical Biomedicine 2(2):S637-S640.

Meena KL, Yadav BL. 2010. Studies on ethnomedicinal plants conserved by Garasia tribes of Sirohi district Rajasthan India. Indian Journal of Natural Product Research 1:500-506. 
Mujumdar AM, Misar AV, Upadhye AS. 2005. Antidiarrhoeal activity of ethanol extract of the bark of Dalbergia lanceolaria. Journal of Ethnopharmacology 102(2):213-216.

Mukti M, Rahmatullah M. 2013. Treatment with aquatic plants by a Bagdi tribal healer of Rajbari District, Bangladesh. Ancient Science of Life 33(1):22-26.

Muralidhara Rao D, Pullaiah T. 2007. Ethnobotanical studies on some rare and endemic floristic elements of Eastern Ghats-Hill Ranges of South East Asia, India. Ethnobotanical Leaflets 11:52-70.

Murthy EN, Sudhakar Reddy C, Reddy KN, Raju VS. 2008. Ethnomedicinal observations from the MahaMutharam and Yamanpally tribal villages of Karimangar, East forest division of Andhra Pradesh, India. Ethnobotanical Leaflets 12:513-551.

Murty PP, Venkaiah M. 2010. Some abortifacient plants used by the tribal people of Andhra Pradesh, India. Journal of Phytology 2(4):7-12.

Muthuswamya R, Rb S. 2014. Pharmacognostical studies on the fruit of Elaeocarpus oblongus Gaertn. Pharmacognosy Journal 6(3):72-78.

Naidu MT, Babu NC, Venkaiah M. 2013. Ethnic remedies gainst snakebite from kotia hills of Vizianagaram district, Andra pradesh, India. Indian Journal of Natural Product Research 4:194-196.

Namsa ND, Tag H, Mandal M, Kalita P, Das AK. 2009. An ethnobotanical study of traditional antiinflammatory plants used by the Lohit community of Arunachal Pradesh, India. Journal of Ethnopharmacology 125(2):234-245.

Nanjunda DC. 2010. Ethno-medico-botanical investigation of Jenu Kuruba ethnic group of Karnataka state, India. Bangladesh Journal of Medical Science 9(3):161-169.

Narayanan MR, Anilkumar N, Balakrishnan V, Sivadasan M, Alfarhan HA, Alatar AA. 2011. Wild edible plants used by the Kattunaikka, Paniya and Kuruma tribes of Wayanad District, Kerala, India. Journal of Medicinal Plants Research 5(15):35203529.

Natarajan B, Paulsen BS. 2000. An ethnopharmacological study from Thane district, Maharashtra, India: Traditional knowledge compared with modern biological science. Pharmaceutical Biology 38(2):139-151.

Nazarudeen A. 2010. Nutritional composition of some lesser-known fruits used by the ethnic communities and local folks of Kerala. Indian Journal of Traditional Knowledge 9(2):398-402.

Nizar KM, Gopakumar S, Vikas Kumar AR. 2015. Indigenous Ethnomedicines and Victuals of Malayans: An Indigenous Population of PeechiVazhani Wildlife Sanctuary, Western Ghats, India. Indian Journal of Ecology 42(1):9-15.
Otieno J, Abihudi S, Veldman S, Nahashon M, vanAndel T, de-Boer HJ. 2015. Vernacular dominance in folk taxonomy: a case study of ethnospecies in medicinal plant trade in Tanzania. Journal of Ethnobiology Ethnomedicine 11(1):1-7.

Owuor BO, Mulemi BA, Kokwaro JO. 2005. Indigenous snake bite remedies of the Luo of western Kenya. Journal of Ethnobiology 25(1):129141.

Oxford Dictionaries. 2013. "ethnicity: definition of ethnicity". Oxford University Press. Oxford, U.K.

Chander MP, Kartick C, Vijayachari P. 2015. Ethnomedicinal knowledge among Karens of Andaman \& Nicobar Islands, India. Journal of Ethnopharmacology 162:127-133.

Padal SB, Sri BS, Satyavathi K. 2014. An ethnomedico botanical survey of plants in North-coastal region of Andhra Pradesh, India. Malaya Journal of Biosciences 1(3):201-206.

Padma Y, Sarojinidevi N, Ratnam KV, Reddy GT, Raju RV. 2016. Herbal/Folk remedies used for snake bites by tribals/rural people of Rayalaseema region, Andhra Pradesh, India. Journal of Medicinal Plants 4(2):52-56.

Pandey A, Negi PS. 2016. Traditional uses, phytochemistry and pharmacological properties of Neolamarckia cadamba: A review. Journal of Ethnopharmacology 181:118-135.

Pandikumar P, Babu NP, Ignacimuthu S. 2009. Hypoglycemic and antihyperglycemic effect of Begonia malabarica Lam. in normal and streptozotocin induced diabetic rats. Journal of Ethnopharmacology 124(1):111-115.

Pani M, Nahak G, Sahu RK. 2014. Review on Ethnomedicinal Plants of Odisha for the Treatment of Malaria. International Journal of Pharmacognosy and Phytochemical Research 7(1):156-165.

Parada M, Carrió E, Bonet MÀ, Vallès J. 2009. Ethnobotany of the Alt Emporda region (Catalonia, Iberian Peninsula): plants used in human traditional medicine. Journal of Ethnopharmacology 124(3):609-618.

Parijatham RT, Sujatha B. 2016. Ethnomedicinal studies of medicinal plants in Eastern ghats of Vizianagaram district, Andhra Pradesh, India. International Journal of Bioassays 5(2):4825-4842.

Patel JJ, Acharya SR, Acharya NS. 2014. Clerodendrum serratum (L.) Moon.-A review on traditional uses, phytochemistry and pharmacological activities Journal of Ethnopharmacology 154:268-285.

Patel DK. 2014. Some Traditional Medicinal Plants Useful for Boil, Burn and for Wounds Healing. Journal of Biodiversity \& Endangered Species 2(4):1-4.

Patel NK. 2010. Ethno-medicinal plants used for amenorrhoea and abnormal menstruation diseases 
in Danta Taluka (Gujarat). Ethnobotanical Leaflets $14: 1-4$.

Patil DA, Ahirrao YA. 2011. Ethnomedicinal Knowledge of Plants used by Local People in Buldhana District of Maharashtra (India). Journal of Ecobiotechnology 3(3):11-17.

Perumal PC, Sowmya S, Pratibha P, Vidya B, Anusooriya P, Starlin T, Ravi S, Gopalakrishnan VK. 2015. Isolation, structural characterization and in silico drug-like properties prediction of a natural compound from the ethanolic extract of Cayratia trifolia (L.). Pharmacognosy Research 7(1):121-125.

Phillips O, Gentry AH. 1993. The useful plants of Tambopata, Peru: II. Additional hypothesis testing in quantitative ethnobotany. Economic Botany 47:3343.

Porte SM. 2014. Overview of Folk Medicine Used for Typhoid in India. International Journal for Research in Ayurveda and Pharmacy 5(2):2019-224.

Prabakaran R, Kumar TS, Rao MV. 2013. Role of non timber forest products in the livelihood of Malayali tribe of Chitteri hills of Southern Eastern Ghats, Tamil Nadu, India. Journal of Applied Pharmaceutical Science 3(5):56-60.

Pradheeps M, Poyyamoli G. 2013. Ethnobotany and utilization of plant resources in Irula villages Sigur plateau, Nilgiri Biosphere Reserve, India. Journal of Medicinal Plant Research 7(6):267-276.

Prakash JW, Raja RA, Anderson NA, Williams C, Regini GS, Bensar K, Rajeev R, Kiruba S, Jeeva S, Das SSM. 2008. Ethnomedicinal plants used by Kani tribes of Agasthiyarmalai biosphere reserve, southern Western Ghats. Indian Journal of Traditional Knowledge 7(3):410-413.

Prasad AD, Shyma TB, Raghavendra MP. 2013. Informant consensus factor and antimicrobial activity of ethno medicines used by the tribes of Wayanad district Kerala. African Journal of Microbiology Research 7(50):5657-5663.

Prasad AD, Shyma TB, Raghavendra MP. 2014. Traditional herbal remedies used for management of reproductive disorders in Wayanad District, Kerala. International Journal of Research in Pharmacy and Chemistry 4(2):333-341.

Prasad PRC, Reddy CS, Raza SH, Dutt CBS. 2008. Folklore medicinal plants of North Andaman Islands, India. Fitoterapia 79(6):458-464.

Prashanth Kumar GM, Shiddamallayya N. 2015. Ethanobotanical Study of Less Known Wild Edible Plants of Hakki Pikki Tribes of Angadihalli, Hassan District, Karnataka. Journal of Medicinal Plants 3(5):80-85.

Prashantkumar P, Vidyasagar GM. 2008. Traditional knowledge on medicinal plants used for the treatment of skin diseases in Bidar district, Karnataka. Indian Journal of Traditional Knowledge 7(2):273-276.
Priya J, Gopalan R. 2014. Ethnomedicinal studies in selected medicinal plants of Dhoni forest, Western Ghats, Kerala. Asian Journal of Pharmaceutical and Clinical Research 7(4):3-6.

Puravankara D, Gopal GV. 2012. Ethnomedicinal information of Kurumba tribes of Kundah taluk, Nilgiris district, Tamil Nadu. Medicinal PlantsInternational Journal of Phytomedicines and Related Industries 4(4):198-205.

Pushpagadan P, Rajasekharan A, Ratheeshkumar PK, Jawahar CR, Radhakrishnan K, Nair CPR, Amma LS, Aicrpe AB. 1990. 'Amrithapala'(Janakia arayalpatra, Joseph \& Chandrasekharan), a new drug from the Kani tribe of Kerala. Ancient Science of Life 9(4):212-214.

Pushpakarani R, Natarajan S. 2014. Ethnomedicines used by Kaniyakaran tribes in Kaniyakumari districtSouthern Western Ghats of Tamil Nadu, India. Journal of Applied Pharmaceutical Science. 4(2):5660.

Pushpangadan P, Atal CK. 1984. Ethno-medicobotanical investigations in Kerala I. Some primitive tribals of Western Ghats and their herbal medicine. Journal of Ethnopharmacology 11(1):59-77.

Ragupathy S, Newmaster SG, Murugesan M, Balasubramaniam V. 2009. DNA barcoding discriminates a new cryptic grass species revealed in an ethnobotany study by the hill tribes of the Western Ghats in southern India. Molecular Ecology Resources 9(s1):164-171.

Rahmatullah M, Rahman A, Haque Z, Mollik AH, Miajee EU, Begum R, Rahman M, Nasrin D, Seraj S, Chowdhury AR, Khatun Z. 2010. A survey of medicinal plants used by folk medicinal practitioners of Station Purbo Para village of Jamalpur Sadar Upazila in Jamalpur district, Bangladesh. AmericanEurasian Journal of Sustainable Agriculture 4(2):122-136.

Rajagopal PL, Premaletha K, Sreejith KR, Chacko IA, Nimisha KV. 2016. A critical review on herbal management of asthma in Kannur district of Kerala state. World Wide Journal of Multidisciplinary Research and Development 2(3):48-52.

Rajan S, Sethuraman M, Baburaj DS. 1997. Plants from the traditional medical system of the Nilgiri tribes. Ancient Science of Life 16(4):360-365.

Rajasab AH, Isaq M. 2004. Documentation of folk knowledge on edible wild plants of North Karnataka. Indian Journal of Traditional Knowledge 3(4):419429.

Rajasekharan S, Latha PG. 2008. Ethnomedicine And Lead Based Product Development A Case Study. Souvenir In National Workshop on GrowerIndustry Linkage for Promotion of Medicinal and Aromatic Plants Cultivation. Cochin, Kerala, India, 73-77.

Rajendran A, Rao NR, Kumar KR, Henry AN. 1997. Some medicinal orchids of Southern India. Ancient Science of Life 17(1):10-14. 
Rajendran A, Ravikumar K, Henry AN. 2000. Plant genetic resources and knowledge of traditional medicine in Tamil Nadu. Ancient Science of Life 20(1-2):25-28.

Rajendran SM, Chandrasekar K, Sundaresan V. 2002. Ethnomedicinal lore of Valaya tribals in Seithur hills of Virudhunagar district, Tamil Nadu, India. Indian Journal of Traditional Knowledge 1:59-71.

Raju RR. 2008. Taxonomic Validation of Crude Drugs used for Poisonous Bites by Adivasis of Rayalaseema Region, Andhra Pradesh. Ethnobotanical Leaflets 12:934-937.

Ram AJ, Bhakshu LM, Raju RV. 2004. In vitro antimicrobial activity of certain medicinal plants from Eastern Ghats, India, used for skin diseases. Journal of Ethnopharmacology 90(2):353-357.

Ram S, Vats A, Sharma AK. 2016. Ethno-Medicinal uses of some plants of Suratgarh Tehsil, Sriganganagar (Rajasthan). Indian Journal of Applied Research 2(4):112-115.

Ramachandran VS. 2007. Wild edible plants of the Anamalais, Coimbatore district, western Ghats, Tamil Nadu. Indian Journal of Traditional Knowledge 6(1):173-176

Ramachandran VS, Joseph S, Aruna R. 2009. Ethnobotanical Studies from Amaravathy Range of Indira Gandhi Wildlife Sanctuary, Western Ghats, Coimbatore District, Southern India. Ethnobotanical Leaflets 13:1069-1087.

Ramya S, Alaguchamy N, Maruthappan VM, Sivaperumal R, Sivalingam M, Krishnan A, Govindaraji V, Kannan K, Jayakumararaj R. 2009. Wound healing ethnomedicinal plants popular among the Malayali tribes in Vattal Hills, Dharmapuri, TN, India. Ethnobotanical Leaflets 13:1257-1271.

Ranjitha MC, Akarsh S, Prashith Kekuda TR, Darshini SM, Vidya P. 2016. Antibacterial activity of some plants of Karnataka, India. Journal of Pharmacognosy and Phytochemistry 5(4):95-99.

Rao CV, Ojha SK, Radhakrishnan K, Govindarajan R, Rastogi S, Mehrotra S, Pushpangadan P. 2004. Antiulcer activity of Utleria salicifolia rhizome extract. Journal of Ethnopharmacology 91(2):243-249.

Rao DM, Rao UVU, Sudharshanam G. 2006. Ethnomedico-botanical studies from Rayalaseema region of southern eastern ghats, Andhra Pradesh, India. Ethnobotanical Leaflets 10:198-207.

Rao JK, Suneetha J, Reddi TS, Kumar OA. 2011. Ethnomedicine of the Gadabas, a primitive tribe of Visakhapatnam district, Andhra Pradesh. International Multidisciplinary Research Journal 1(2):10-14.

Rao VLN, Bharathi K, Appalanaidu P, Naidu JM, Venkaiah M. 2012. Common plants of medicinal values in kolams of Adilabad district, Andhra Pradesh. International Journal of Medicine and Biomedical Research 1(2):111-118.
Ratna Manjula R, Rao JK, Reddi, TS. 2011. Ethnomedicinal plants used to cure jaundice in Kammam district of Andhra Pradesh, India. Journal of Phytology 3(10):33-35.

Ratnam KV, Raju RV. 2008. Folk remedies for insect bites from Gundla Brahmeswaram wildlife sanctuary, Andhra Pradesh. Indian Journal of Traditional Knowledge 7:436-437.

Ravishankar T. 2007. Ethnobotany of Dharmapuri and North Arcot Districts in Tamil Nadu, South India. Biodiversity 8(1):12-20.

Reddy A, Reddy PR. 2008. Occurrence of Medicinal Plant Pollen in Apis cerana Honeys of Khammam District, Andhra. Ethnobotanical Leaflets 12:452460.

Reddy KN, Reddy CS, Raju VS. 2008. Ethnomedicinal observations among the Kondareddis of Khammam District, Andhra Pradesh, India. Ethnobotanical leaflets 12:916-926.

Reddy MB, Reddy KR, Reddy MN. 1991. Ethnobotany of Cuddapah district, Andhra Pradesh, India. International Journal of Pharmacognosy 29(4):273-280.

Rekka R, Kumar SS. 2014. Ethnobotanical notes on wild edible plants used by Malayali tribals of Yercaud hills, Eastern Ghats, Salem District, Tamil Nadu. International Journal of Herbal Medicine 2(1):39-42.

Rodrigues E, Carlini EA. 2005. Ritual use of plants with possible action on the central nervous system by the Krahô Indians, Brazil. Phytotherapy Research 19(2):129-135.

Ross IA. 2003. Jatropha curcas. In Medicinal Plants of the World. IA Ross, Humana Press, Totowa, NJ, 277-288.

Ruba AA, Mohan VR. 2016. In Vitro Antioxidant Potential of Andrographis Echioides Whole Plant (Acanthaceae). International Journal of Pharmacy and Pharmaceutical Sciences 8(6):70-75.

Sai Vishnu Priya K, Rao S. 2008. Exploration of Tribal Knowledge of Entada pursaetha DC: An Endangered Gigantic Medicinal Legume in Eastern Ghats. Ethnobotanical Leaflets 12:36-43.

Samy RP, Thwin MM, Gopalakrishnakone P, Ignacimuthu S. 2008. Ethnobotanical survey of folk plants for the treatment of snakebites in Southern part of Tamilnadu, India. Journal of Ethnopharmacology 115(2):302-312.

Saravanan S, Mutheeswaran S, Saravanan M, Chellappandian M, Paulraj MG, Raj MK, Ignacimuthu S, Duraipandiyan V. 2013. Ameliorative effect of Drynaria quercifolia (L.) J. Sm., an ethnomedicinal plant, in arthritic animals. Food and Chemical Toxicology 51:356-363.

Sarvalingam A, Rajendran A, Aravindhan V. 2011. Curative climbers of Maruthamalai hills in the southern Western Ghats of Tamil Nadu, India. 
International Journal of Medicinal and Aromatic Plants 1(3):326-332.

Sautron C, Cock IE. 2014. Antimicrobial activity and toxicity of Syzygium australe and Syzygium leuhmannii fruit extracts. Pharmacognosy Communications 4(1):53-60.

Savithramma N, Yugandhar P, Haribabu R, Prasad KS. 2014. Validation of Indigenous Knowledge of Yanadi Tribe and Local Villagers of VeyilingalakonaA Sacred Grove of Andhra Pradesh, Indian. Journal of Pharmaceutical Sciences and Research 6:382388.

Sayed NZ, Deo R, Mukundan U. 2007. Herbal remedies used by Warlis of Dahanu to induce lactation in nursing mothers. Indian Journal of Traditional Knowledge 6:602-605.

Seraj S, Jahan FI, Chowdhury AR, Monjur-Ekhuda M, Khan MSH, Aporna SA, Jahan R, Samarrai W, Islam F, Khatun Z, Rahmatullah M. 2013. Tribal formulations for treatment of pain: A study of the Bede community traditional medicinal practitioners of Porabari Village in Dhaka District, Bangladesh. African Journal of Traditional, Complementary and Alternative Medicines 10(1):26-34.

Shah B, Sheth F, Parabia M. 2011. Documenting Grandmas' prescriptions for skin ailments in Valsad district, Gujarat. Indian Journal of Traditional Knowledge 10(2):372-374.

Shah RK. 2015. A Review on Ethnobotanical uses of Smilax ovalifolia. International Journal of Herbal Medicine 3(2 Part A):16-19.

Shanmugam S, Rajendran K, Suresh K. 2012. Traditional uses of medicinal plants among the rural people in Sivagangai district of Tamil Nadu, Southern India. Asian Pacific Journal of Tropical Biomedicine 2(1):S429-S434.

Sharma J, Gairola S, Gaur RD, Painuli RM, Siddiqi TO. 2013. Ethnomedicinal plants used for treating epilepsy by indigenous communities of subHimalayan region of Uttarakhand, India. Journal of Ethnopharmacology 150(1):353-370.

Sharma J, Painuli RM, Gaur RD. 2010. Plants used by the rural communities of district Shahjahanpur, Uttar Pradesh. Indian Journal of Traditional Knowledge 9(4):798-803.

Sharma PK. 2009. Studies on ethnomedicinal uses of herbal plants resources in northern hilly zone of Chhattisgarh. International Journal of Plant Science 5(1):158-161.

Sharma PP, Mujundar AM. 2003. Traditional knowledge on plants from Toranmal Plateau of Maharastra. Indian Journal of Traditional Knowledge 2:292-296.

Sheikh N, Kumar Y, Misra, AK, Pfoze L. 2013. Phytochemical screening to validate the ethnobotanical importance of root tubers of Dioscorea species of Meghalaya, North East India. Journal of Medicinal Plants 1(6):62-69.
Singh AG, Kumar A, Tewari DD. 2012. An ethnobotanical survey of medicinal plants used in terai forest of western Nepal. Journal Ethnobiology and Ethnomedicine 8(19):1-15.

Singh N. 2015. Understanding poor man's diseases in contemporary perspective. The Indian Journal of Medical Research 141(5):501-504.

Sivalingam D, Rajendran R, Anbarasan K. 2016. Ethnopharmacological values of Sacred Trees of Big Temples in Cuddalore district, Tamil Nadu, India. Bulletin of Environment, Pharmacology and Life Sciences 5:39-46.

SM SM, Vidyasagar GM. 2013. Medicinal plants used in the treatment of gastrointestinal disorders in Bellary district, Karnataka, India. Indian Journal of Traditional Knowledge 12(2):321-325.

Sreeramulu N, Suthari S, Ragan A, Raju VS. 2013. Ethno-botanico-medicine for common human ailments in Nalgonda and Warangal districts of Telangana, Andhra Pradesh, India. Annals of Plant Sciences 2(07):220-229.

Sripathi SK, Sankari U. 2010. Ethnobotanical documentation of a few medicinal plants in the Agasthiayamalai region of Tirunelveli District, India. Ethnobotanical Leaflets 14:173-181.

Srithi $\mathrm{K}$, Balslev $\mathrm{H}$, Wangpakapattanawong $\mathrm{P}$, Srisanga P, Trisonthi C. 2009. Medicinal plant knowledge and its erosion among the Mien (Yao) in northern Thailand. Journal of Ethnopharmacology 123(2):335-342.

Subramanian R, Krishnaswamy G, Devaraj A, Sethuraman P, Jayakumarara R. 2011. Wound healing ethnopharmacological potentials of selected medicinal plants used by Malayali Tribes. International Research Journal of Pharmacy 2:132137.

Subramanian S, Ramakrishnan N. 2011. Chromatographic finger print analysis of Naringi crenulata by HPTLC technique. Asian Pacific Journal of Tropical Biomedicine 1(2):S195-S198.

Sudhadevi PK. 1999. Folkloric plant remedies for Rheumatism. Ancient Science of Life 18(3-4):264265.

Suganthi A, Libina S. 2015. Survey of medicinal and anticancer plants from Vengad Sree Kurumba Kavu sacred grove, Kannur Kerala. Journal of Medicinal Plant Research 3(6):46-50.

Sugumaran P, Kowsalya N, Karthic R, Seshadri S. 2013. Biomass production and antibacterial activity of Justicia gendarussa Burm. f.-A valuable Medicinal plant. Journal of Tropical Life Science 3(1):8-13.

Sulochana AK, Raveendran D, Krishnamma AP, Oommen OV. 2015. Ethnomedicinal plants used for snake envenomation by folk traditional practitioners from Kallar forest region of South Western Ghats, Kerala, India. Journal of Intercultural Ethnopharmacology 4(1):47-51. 
Suneetha J, Rao JK, Reddi TS. 2011. Phytotherapy for Dyspepsia by the Tribals of East Godavari District, Andhra Pradesh. Journal of Phytology 3(6):63-65.

Suresh V, Asha VV. 2008. Preventive effect of ethanol extract of Phyllanthus rheedii Wight. on Dgalactosamine induced hepatic damage in Wistar rats. Journal of Ethnopharmacology 116(3):447-453.

Surveswaran S, Cai YZ, Xing J, Corke H, Sun, M. 2010. Antioxidant properties and principal phenolic phytochemicals of Indian medicinal plants from Asclepiadoideae and Periplocoideae. Natural Product Research 24(3):206-221.

Suthari S, Raju VS. 2016. Antidote Botanicals for Snake Bites from Koyas of Warangal District, Telangana, India. Journal of Herbs, Spices \& Medicinal Plants 22(1):57-68.

Swarnkar S, Katewa SS. 2008. Ethnobotanical observation on tuberous plants from tribal area of Rajasthan (India). Ethnobotanical Leaflets 12:647666.

Tetali P, Waghchaure C, Daswani PG, Antia NH, Birdi TJ. 2009. Ethnobotanical survey of antidiarrhoeal plants of Parinche valley, Pune district, Maharashtra, India. Journal of Ethnopharmacology 123(2):229-236.

Thirunarayanan T. 2013. Ethnobotanical survey on Folk Medicine in the management of animal bite poisons in the forest tract of Salem region of Tamil Nadu, India. International Journal of Pharmacology and Clinical Sciences 2(2):41-46.

Trotter RT, Logan MH. 1986. Informant census: a new approach for identifying potentially effective medicinal plants. In Plants in indigenous medicine and diet: Biobehavioral approaches. Edited by NL Etkin. Redgrave Publishing Comapny, Bedford Hill, New York, 91-112.

Udayan PS, George S, Tushar KV, Balachandran I. 2005. Medicinal plants used by the Kaadar tribes of Sholayar forest, Thrissur district, Kerala. Indian Journal of Traditional Knowledge 4(2):159-163.

Udayan PS, George S, Tushar KV, Balachandran I. 2007. Ethnomedicine of Malapandaram tribes of Achenkovil forest of Kollam district, Kerala. Indian Journal of Traditional Knowledge 6:569-573.

Umapriya T, Rajendran A, Aravindhan V, Thomas B, Maharajan M. 2011. Ethnobotany of Irular tribe in Palamalai Hills, Coimbatore, Tamil Nadu. Indian Journal of Natural Products and Resources 2(2):250255.

UNHR \& APF. 2013. The United Nations Declaration on the Rights of Indigenous Peoples. A Manual for National Human Rights Institutions. United Nations High Commissioner for Human Rights (OHCHR): Australia.

United Nations Report. 2009. State of the World's Indigenous Peoples. Department of Economic and Social Affairs, Secretariat of the Permanent Forum on Indigenous Issues, United Nations, New York, 1237.

Upadhya V, Hegde HV, Bhat S, Hurkadale PJ, Kholkute SD, Hegde GR. 2012. Ethnomedicinal plants used to treat bone fracture from North-Central Western Ghats of India. Journal of Ethnopharmacology 142(2):557-562.

Valsaraj R, Pushpangadan P, Smitt UW, Adsersen A, Nyman U. 1997. Antimicrobial screening of selected medicinal plants from India. Journal of Ethnopharmacology 58(2):75-83.

Vedavathy S, Sudhakar A, Mrdula, V. 1997. Tribal medicinal plants of Chittoor. Ancient Science of Life 16(4):307-331.

Vijaykumar MMJ, Taranath TC. 2010. Survey on Ethno medicinal Plants of Jogimatti Forest Chitradurga District, Karnataka, India. Environment wq\& We: An International Journal of Science \& Technology 5:223-233.

Vilash V, Suja SR, Latha PG, Shine VJ, Rajasekharan S. 2016. Chronic oral toxicity studies of crude ethanolic extract and ethanolic fraction of Pellionia heyneana Wedd. leaf in wistar rats. International Journal of Pharmacy and Pharmaceutical Sciences 8(8):306-312.

Wagh VV, Jain AK. 2015. Inventory of ethnobotanicals and other systematic procedures for regional conservation of medicinal and sacred plants. Environment Systems and Decisions 35(1):143-156.

Wills PJ, Asha VV. 2006. Protective effect of Lygodium flexuosum (L.) Sw.(Lygodiaceae) against D-galactosamine induced liver injury in rats. Journal of Ethnopharmacology 108(1):116-123.

World Health Organization. 2013. WHO traditional medicine strategy: 2014-2023. WHO Press, World Health Organization, Geneva, Switzerland.

Xavier TF, Kannan M, Lija L, Auxillia A, Rose AKF. 2014. Ethnobotanical study of Kani tribes in Thoduhills of Kerala, South India. Journal of Ethnopharmacology 152(1):78-90.

Xaxa V. 1999. Tribes as indigenous people of India. Economic and Political Weekly 3589-3595.

Yazdanshenas $\mathrm{H}$, Tavili A, Arzani $\mathrm{H}$, Azarnivand $\mathrm{H}$. 2016. News and Views Article Traditional Gundelia tournefortii Usage and its Habitat Destruction in Tiran va Karvan District in Iran's Isfahan Province. Ecologia 6(1-3):19-25.

Yesodharan K, Sujana KA. 2007. Ethnomedicinal knowledge among Malamalasar tribe of Parambikulam wildlife sanctuary, Kerala. Indian Journal of Traditional Knowledge 6(3):481-485.

Zakaria ZA, Ghani ZDFA, Nor RNSRM, Gopalan HK, Sulaiman MR, Jais AMM, Somchit MN, Kader AA, Ripin J. 2008. Antinociceptive, anti-inflammatory, and antipyretic properties of an aqueous extract of 
Dicranopteris linearis leaves in experimental animal models. Journal of Natural Medicines 62(2):179-187.

Zent S, Zent EL. 2007. On biocultural diversity from a Venezuelan perspective: Tracing the interrelationships among biodiversity, culture change and legal reforms. In Biodiversity \& the Law: Intellectual Property, Biotechnology \& Traditional Knowledge. Edited by CR McManis. Earthscan, London, U.K., 91-114.

Zhang L, Ravipati AS, Koyyalamudi SR, Jeong SC, Reddy N, Bartlett J, Smith PT, de la Cruz M, Monteiro MC, Melguizo Á, Jiménez E. 2013. Anti-fungal and anti-bacterial activities of ethanol extracts of selected traditional Chinese medicinal herbs. Asian Pacific Journal of Tropical Medicine 6(9):673-681. 